\title{
In-situ Zugversuche an einkristallinen Gold-Nanodrähten im TEM
}

\author{
Dissertation \\ zur Erlangung des mathematisch-naturwissenschaftlichen Doktorgrades \\ Doctor rerum naturalium \\ der \\ Georg-August-Universität Göttingen
}

vorgelegt von

Burkhard Roos

aus Lübeck

Göttingen, 2012 
Referentin: Prof. Cynthia A. Volkert

Korreferent: apl. Prof. Michael Seibt

Tag der mündlichen Prüfung: 19. Juli 2012 


\section{Inhaltsverzeichnis}

Abbildungsverzeichnis $\quad$ v

Tabellenverzeichnis $\quad$ ix

1 Einleitung 1

1.1 Stand der Forschung . . . . . . . . . . . . . . . . 3

1.1.1 Motivation der Arbeit . . . . . . . . . . . . . . 8

2 Theoretische Grundlagen $\quad 11$

2.1 Kristallographie und Verformung . . . . . . . . . . . . . . . . . 11

2.1.1 Versetzungsnukleation und Versetzungstypen . . . . . . . . . 12

2.1.2 Das Schmidsche Schubspannungsgesetz . . . . . . . . . . . 16

2.1.3 Mechanismen der Zwillingsbildung . . . . . . . . . . 17

2.1.4 Kristallographie der Zugversuche . . . . . . . . . . . . . . . 19

2.2 Elektronenbeugung und reziproker Raum . . . . . . . . . . . . . . 20

2.2.1 Beugung von dünnen Probenbereichen . . . . . . . . . . . . . . . 24

2.3 Die Howie-Whelan Gleichungen . . . . . . . . . . . . . . . . . . 26

2.4 Charakteristische Kontraste im TEM . . . . . . . . . . . . . . 27

2.4 Biegekontrast . . . . . . . . . . . . . . 27

2.4.2 Kontrast von planaren Defekten . . . . . . . . . . . 28

2.4.3 Versetzungskontrast im TEM . . . . . . . . . . . . . . . . 31

3 Die Nanodrähte 33

3.1 Vorcharakterisierung im REM . . . . . . . . . . . . . . . . . . 34

3.2 Vorcharakterisierung im TEM . . . . . . . . . . . . . . . 35

3.2.1 Überstruktur in der $\{111\}$-Zonenachse . . . . . . . . . . . . 38 
4 Experimentelle Grundlagen $\quad 41$

4.1 Vorpräparation von Verformungsproben . . . . . . . . . . . . . 41

4.2 Zugversuche auf CuBe-Rahmen . . . . . . . . . . . . . . . 42

4.2 .1 Co-Deformation . . . . . . . . . . . . . . . . . 44

4.2 .2 Ablauf des Zugversuches . . . . . . . . . . . . . . . 48

4.2.3 Getestete Drähte im Überblick . . . . . . . . . . . . . . . . . . . 49

4.2.4 Defektgeschwindigkeit . . . . . . . . . . . . . . . . . 49

4.3 Biegeversuche . . . . . . . . . . . . . . . . 52

5 Ergebnisse $\mathbf{5 5}$

5.1 Zugversuche mit Stapelfehlern und Zwillingen . . . . . . . . . . . . 55

5.1 .1 Verlauf der Defektdichte . . . . . . . . . . . . 56

5.1 .2 Analyse der Stapelfehler . . . . . . . . . . . . . . . . . 61

5.1.2.1 Interaktion von Stapelfehlern . . . . . . . . . . . . 66

5.1 .3 Analyse der Zwillinge . . . . . . . . . . . . . . . . 67

5.1 .4 Dehnungsabschätzung . . . . . . . . . . . . . 71

5.1 .5 Bruchverhalten . . . . . . . . . . . . . 73

5.1 .6 Länge des Verformungsbereiches . . . . . . . . . . . . 76

5.1.7 Einfluss des Elektronenstrahls . . . . . . . . . . . . . . . 79

5.1 .8 Entlastungsversuch . . . . . . . . . . . . . . . . . . . 81

5.2 Zugversuche mit vollen Versetzungen . . . . . . . . . . . . 83

5.2 .1 Bruchverhalten . . . . . . . . . . . . . . 88

5.2 .2 Defektdichte . . . . . . . . . . . . . . . . . . . . 92

5.3 Einfluss von FIB-Schädigung auf den Verformungsmechanismus . . . . . 93

5.3.1 Präparation und Galliumkonzentration . . . . . . . . . . . . . 94

5.3.2 Verformungsverhalten der geschädigten Drähte . . . . . . . . . 98

5.4 Biegeversuche . . . . . . . . . . . . . . . . . . . . 100

5.5 Vergleich der Verformungsexperimente . . . . . . . . . . . . . . . 108

5.5.1 Drahtgröße und Defekttyp . . . . . . . . . . . . . . . 108

5.5.2 Überstruktur und Defekttyp . . . . . . . . . . . . . . . . . . 110

5.5.3 Zwillingsbildung und Defekttyp . . . . . . . . . . . 111

5.5.4 Spannungszustand und Defekttyp . . . . . . . . . . . . 113 
6 Diskussion $\quad 115$

6.1 Zum Auftreten von Partialversetzung . . . . . . . . . . . . . . . 116

6.1.1 Auspaltungsweite von Partialversetzungen . . . . . . . . . . . . 117

6.1.2 Einführung in die Konzepte der Generalized Stacking Fault Energy 122

6.1.2.1 Generalized Stacking Fault Energy . . . . . . . . . . . 124

6.1.3 Klassische Keimbildungstheorie . . . . . . . . . . . . . . . . . . 131

6.1.4 Analyse der Zwillingsbildung . . . . . . . . . . . . . . . . 146

6.1.5 Galliumschädigung . . . . . . . . . . . . . . . . . . . . . 148

6.1.6 Einfluss des Spannungszustandes . . . . . . . . . . . . . . 149

6.1.7 Einflüsse der Mikrostruktur auf die Versetzungsbewegung . . . . 151

6.1.8 Kombination der vorliegenden Modelle . . . . . . . . . . . . . . . 154

$\begin{array}{llr}7 & \text { Fazit und Ausblick } & 157\end{array}$

$\begin{array}{lc}\text { Literatur } & 163\end{array}$ 
INHALTSVERZEICHNIS 


\section{Abbildungsverzeichnis}

1.1 Fließspannung von einkristallinen Kupfer-Drähten ... . . . . . . . . 2

1.2 Verlauf der Festigkeit einer metallischen Probe . . . . . . . . . . . . 3

1.3 TEM Aufnahmen von verformten Gold-Säulen . . . . . . . . . . . . . . . 4

1.4 Wechsel des Verformungsmechanismus mit der Schichtdicke . . . . . . . 5

1.5 Atomar aufgelöste Verformung von Goldkontakten . . . . . . . . . . . 6

1.6 MD Simulation der Verformung eines Gold-Nanodrahtes . . . . . . . . . 7

2.1 Schemazeichnung der fcc Einheitszelle . . . . . . . . . . . . . . 12

2.2 Schemazeichnung einer $\{111\}$-Ebene in einem fcc Gitter . . . . . . . . 14

2.3 Typen von Stapelfehlern in fcc . . . . . . . . . . . . . . . 16

2.4 Skizze zur Veranschaulichung des Schmidfaktors . . . . . . . . . . 17

2.5 Schematische Darstellung eines Zwillings in fcc . . . . . . . . . . 18

2.6 Schematische Zeichung der Zwillingsbildung . . . . . . . . . . 20

2.7 Kristallographie eines Zugversuches in [110]-Richtung . . . . . . . . . . . 21

2.8 Geometrie der Beugung im reziproken Raum . . . . . . . . . . . . . . 23

2.9 Beugung von einer keilförmigen Probe . . . . . . . . . . . . . 25

2.10 schematische Darstellung zum Auftreten von Biegekontrasten . . . . . . 27

2.11 Stapelfehler in einem Gold Nanodraht . . . . . . . . . . . . . . 30

2.12 Schematische Zeichung und Beispiele zum Kontrastmechanismus einer

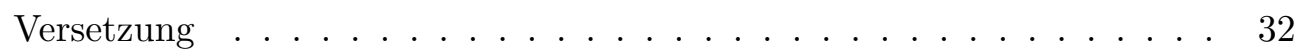

3.1 REM-Aufnahme der Gold-Nanodrähte . . . . . . . . . . . . . . . . . 34

3.2 TEM Aufnahmen zur Orientierungsbestimmung der Gold-Drähte . . . . 37

3.3 Breitenbestimmung der Drähte . . . . . . . . . . . . . 38

3.4 Beugungsbilder der (111)-Zonenachse . . . . . . . . . . . . . . . 39 
4.1 Vorbereitende Schritte zum Drahttransfer . . . . . . . . . . . . . . 42

4.2 Vorbereiteter CuBe Rahmen . . . . . . . . . . . . . . . . . . . . 44

4.3 Prozessschritte zur Präparation eines Zugversuches . . . . . . . . . . . . 44

4.4 Zeichnung des Zughalters . . . . . . . . . . . . . . . . . 45

4.5 Schemazeichnung des CuBe-Rahmens . . . . . . . . . . . . 46

4.6 FE-Simulation der Verformung eines CuBe Rahmens . . . . . . . . . . . 47

4.7 Vorgeschnittenes TEM-Netzchen für einen Biegeversuch . . . . . . . . . 52

4.8 Präparation eines Biegeversuches . . . . . . . . . . . . . . 53

4.9 Schemazeichnung des STM-Halters . . . . . . . . . . . . . . . 54

5.1 Entwicklung der Defektanzahl . . . . . . . . . . . . . . . . 57

5.2 Beginn der plastischen Verformung während des Zugversuches . . . . . . 58

5.3 Fortschreitende Verformung . . . . . . . . . . . . . . . 58

5.4 Maximale Defektdichte kurz vor Reissen des Drahtes . . . . . . . . . . 59

5.5 Defektverteilung . . . . . . . . . . . . . . . . 60

5.6 Abstandsverteilung der Stapelfehler . . . . . . . . . . . . . . . 62

5.7 „Streaking“ der Beugungsreflexe durch Stapelfehler . . . . . . . . . . . . 63

5.8 Quantitative Analyse eines Stapelfehlers . . . . . . . . . . . . . . . . 64

5.9 Planare Defekte, die die Querschnittsform des Drahtes wiedergeben . . . 65

5.10 Zick-Zack-Positionierung von sich nicht durchdringenden Stapelfehlern . 66

5.11 Nicht durchlaufende Stapelfehler . . . . . . . . . . . . . . . . . 67

5.12 Zwillingsnachweis in einem Draht über den Kontrastwechsel . . . . . . . 68

5.13 Durch Verformung entstandener Zwilling . . . . . . . . . . . . . . . 69

5.14 Zwillingswachstum . . . . . . . . . . . . . . . . 70

5.15 Ermittlung der Dehnung zwischen Markerdefekten in einem $60 \mathrm{~nm}$ Draht 71

5.16 Bruchverhalten eines Drahtes mit Zwillingen . . . . . . . . . . . . . 74

5.17 Zwillingsgeometrie . . . . . . . . . . . . . . . . . . . 74

5.18 Übersicht über den Verformungsbereich . . . . . . . . . . . . . . . 77

5.19 Schematische Darstellung der Platinschicht . . . . . . . . . . . . 77

5.20 Einfluss des Elektronenstrahls . . . . . . . . . . . . . . . . 80

5.21 Hellfeldbilder des Entlastungsversuchs . . . . . . . . . . . . . . . 82

5.22 Vergleich der Verformungsbereiche . . . . . . . . . . . . . . . 84

5.23 Defekte während des Zugversuches ． . . . . . . . . . . . . 85 
5.24 Oberflächenstufen im TEM . . . . . . . . . . . . . . . . 86

5.25 Hellfeldaufnahmen eines $190 \mathrm{~nm}$ Drahtes während des Zugversuches . 86

5.26 Verformung eines $300 \mathrm{~nm}$ Drahtes . . . . . . . . . . . . . 87

5.27 Bruchverhalten von Drähten mit vollen Versetzungen . . . . . . . . . . 88

5.28 Verjüngung an Bruchkanten zweier verformter Drähte . . . . . . . . 89

5.29 Übersichtsbild eines gerissenen Drahtes . . . . . . . . . . . . . . . . . 91

5.30 Skizze des Ga-Implantationsexperimentes . . . . . . . . . . . . . . . 94

5.31 SRIM Simulation zu Gallium in Gold . . . . . . . . . . . . . . . . . 96

5.32 Gold-Gallium Phasendiagramm . . . . . . . . . . . . . . . . . . . . 97

5.33 Gallium-Schädigung . . . . . . . . . . . . . . . . . . . . 98

5.34 Defekte und Bruchkante bestrahlter Drähte . . . . . . . . . . . . . 99

5.35 Mikrostruktur des Drahtes beim ersten Biegeversuch . . . . . . . . . . 101

5.36 Quantitative Abschätzung der Spannung . . . . . . . . . . . . . 102

5.37 Schemazeichung der Verbiegung . . . . . . . . . . . . . . . . . 102

5.38 Der zweite Biegeversuch . . . . . . . . . . . . . . . . . 103

5.39 Versetzungen in einem verbogenen $110 \mathrm{~nm}$ Draht . . . . . . . . . . . 104

5.40 Dritter Biegeversuch . . . . . . . . . . . . . . . . . 106

5.41 Defekte beim dritten Biegeversuch . . . . . . . . . . . . . . 106

5.42 Größenabhängigkeit der Defekttypen . . . . . . . . . . . . . . . . . . 109

5.43 Verformter $65 \mathrm{~nm}$ Draht . . . . . . . . . . . . . . . . . . 112

6.1 Zeichnung der Aufspaltungsgeometrie . . . . . . . . . . . . . . . 118

6.2 Winkelabhängigkeit der Aufspaltungsweite . . . . . . . . . . . . . . . 119

6.3 Verlauf der Aufspaltungsweite in Abhängigkeit einer angelegten Scherspannung . . . . . . . . . . . . . . . . . 120

6.4 GSFE Kurve . . . . . . . . . . . . . . . . . . . . . . 125

6.5 Potentialverlauf für die Bildung der Partialversetzungen mit unterschiedlichen Richtungen . . . . . . . . . . . . . . . . . . . . 126

6.6 Potentialverlauf für die Zwillingsbildung . . . . . . . . . . . . . 127

6.7 GSFE Vorhersage der Verformung . . . . . . . . . . . . . . . 129

6.8 Kritischer Keimradius im elastischen Modell . . . . . . . . . . . . . . . . 132

6.9 Kritische Keimradien der Versetzungsnukleation . . . . . . . . . . . 135

6.10 Energie der kritischen Versetzungskeime . . . . . . . . . . . . . 137 
6.11 Nukleationsraten für Partialversetzungen . . . . . . . . . . . . 140

6.12 Untersuchung der Defekte auf statistische Verteilung . . . . . . . . . . 145

6.13 Schemazeichnung des Einflusses einer Zwillinsglamelle . . . . . . . . . . 147

6.14 Nukleationsraten im Druckversuch ． . . . . . . . . . . . . . . 150

6.15 Atomare Darstellung sich schneidender Stapelfehler . . . . . . . . . . . . 152

6.16 Spannungsabhängiger Verlauf der GSFE . . . . . . . . . . . . 155 


\section{Tabellenverzeichnis}

2.1 Schmidfaktoren . . . . . . . . . . . . . . . . . . . 22

4.1 Zugversuche im Überblick . . . . . . . . . . . . . . . . . . . . 50

5.1 Schmidfaktoren im Zwilling . . . . . . . . . . . . . . . . . . . . . . . . . . . .

5.2 Verformungsbereich . . . . . . . . . . . . . . . 83

5.3 Biegeversuche . . . . . . . . . . . . . . . . . . . 100

5.4 Einfluss der Überstruktur auf die Verformung . . . . . . . . . . . . . 111

5.5 Kristallografischer Einfluss auf die Zwillingsbildung . . . . . . . . . . . 113

5.6 Einfluss der Belastungsart auf den Defekttyp . . . . . . . . . . . . . . 113 


\section{1}

\section{Einleitung}

Als in den 1950er Jahren Brenner in seiner viel zitierten Arbeit die Fließspannung von nur einige Mikrometer großen metallischen Drähten vorstellte [1], war dies ein wesentlicher Grundstein für die Untersuchung von größenabhängigen mechanischen Eigenschaften in kleinskaligen Proben, der bis heute große Relevanz hat. Abbildung 1.1 zeigt die in dieser Arbeit ermittelte größenabhängige Fließspannung von Kupfer-Drähten. Man erkennt in dieser Darstellung, dass die Drähte einen ausgeprägten Größeneffekt zeigten und Fließspannungen aufwiesen, die nahe der theoretischen Festigkeit lagen. Brenner konnte durch eine systematische Analyse der Daten einige wichtige Faktoren für die Verformung in diesen Proben ableiten. So erkannt er zum Beipsiel, dass offensichtliche Defekte an der Oberfläche die Festigkeit massiv negativ beeinflussten. Ebenso führten mikrostrukturelle Einflüsse, wie Korngrenzen, dazu, dass die Festigkeit stark abnahm. Die besondere Tragkraft erlangte die Arbeit aber vor allem deswegen, weil Brenner in der Lage war, etablierte Modelle für die Erklärung der hohen Festigkeit an seinen Proben auszuschließen. So war eine Erklärung über hohe Versetzungsdichten [2], kleine Korngrößen [3] oder Fremdatome [4] nicht anwendbar für die Proben. Diese Faktoren wirken als Hindernisse für die Versetzungsbewegung und haben somit direkt einen Einfluss auf die Festigkeit. Nimmt der mittlere Abstand zwischen den Hindernissen zu, wird die freie Laufweite der Versetzung klein und die Festigkeit steigt an. Der Grenzfall eines perfekten Einkristalls konnte also mit keiner Vorhersage dieser Modelle in Einklang gebracht werden. Diese Tatsache ist schematisch in Abbildung 1.2 für den Fall der Versetzungsdichte dargestellt. Mit den Experimenten von Brenner wurde folglich ein ganz neuer Aspekt der Verformung in den Fokus gerückt: Plastische Dehnung, 


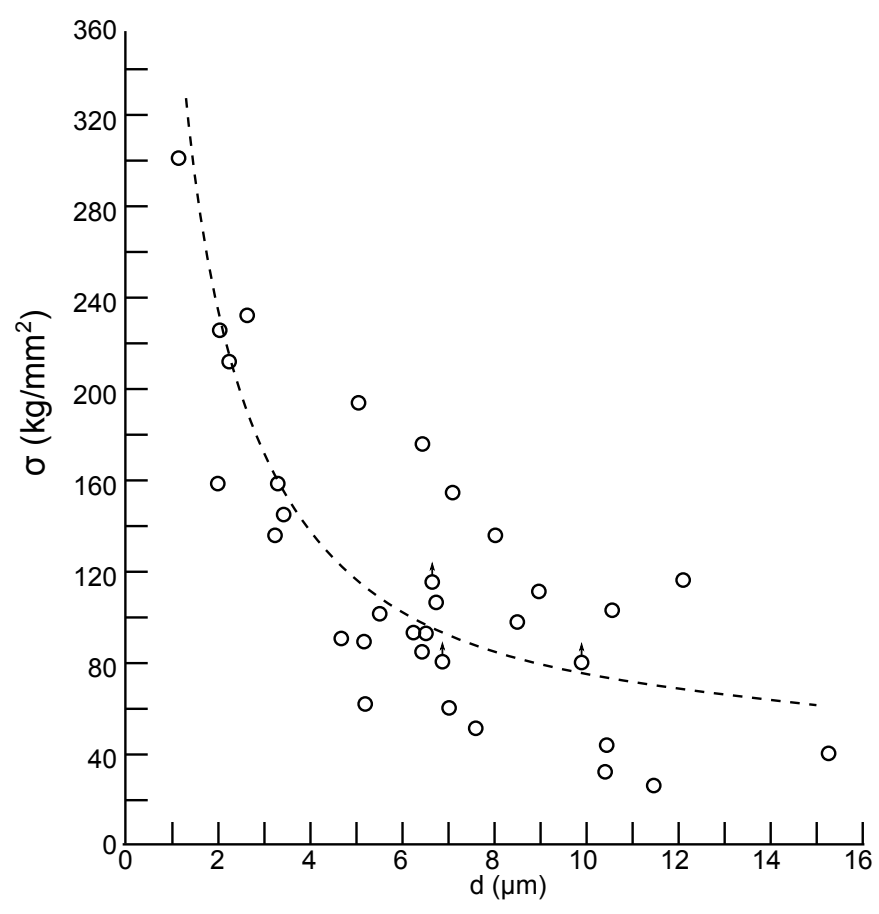

Abbildung 1.1: Fließspannung von einkristallinen Kupfer-Drähten - Auftragung der im Zugversuch ermittelten Fließspannung von einkristallinen Kupfer-Drähten gegen ihren Durchmesser (entnommen aus [1]).

die allein durch Keimbildung von Versetzungen limitiert ist. Brenner konnte in seiner Arbeit noch keine genaues Bild für diesen Vorgang liefern, das den Größeneffekt in der Verformung erklärt. Jedoch war er bereits in der Lage einige kritische Faktoren zu bestimmen. So erkannte er, dass die Abhängigkeit der Festigkeit von dem reziproken Durchmesser der Probe ein Indiz dafür war, dass entweder die Oberfläche oder bereits im Volumen vorhandene Quellen ausschlaggebend waren.

Im folgenden Abschnitt soll nun der aktuelle Stand der Forschung rekapituliert werden, der sich mit diesen Aspekten der Verformung auseinander setzt. Anhand ausgewählter Beispiele soll aufgezeigt werden, welche Untersuchungen bereits unternommen worden sind, um ein Verständnis von den größenabhängigen mechanischen Eigenschaften und der Versetzungskeimbildung zu erlangen. Ausgehend von dieser Zusammenfassung folgt dann die Formulierung einer neuen experimentellen Herangehensweise, die die vorliegende Arbeit motiviert. 


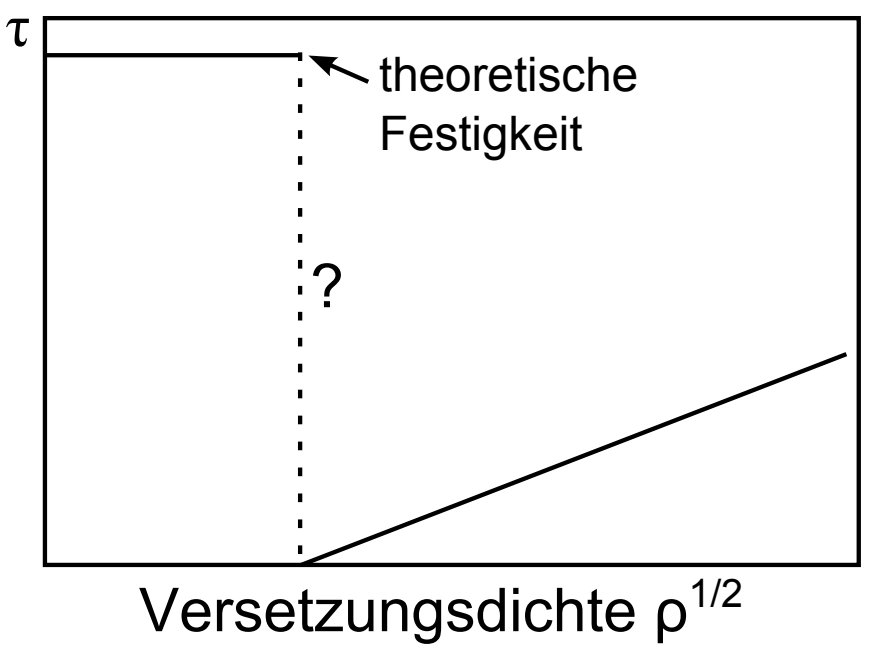

Abbildung 1.2: Verlauf der Festigkeit einer metallischen Probe - Schematischer Verlauf der Festigkeit einer metallischen Probe am Beispiel der Versetzungsdichte(nach [5])

\subsection{Stand der Forschung}

Die Wahl von Gold als Material für die Untersuchung der mechanischen Eigenschaften hat große Vorteile. Das epitaktische Wachstum von Gold ist hinreichend gut verstanden, um qualitativ hochwertige einkristalline Schichten herzustellen. Auch hat Gold als Edelmetall die Eigenschaft keine Oxidschicht zu bilden, was den Einfluss einer chemisch veränderten Oberfläche im Experiment ausschließt [6]. Allerdings erweist sich als nachteilig, dass bedingt durch die hohe Ordnungszahl die Durchstrahlbarkeit im Transmissionselektronenmikroskop (TEM) auf sehr dünne Proben beschränkt ist. Dennoch wurde Gold schon in einer Vielzahl von Geometrien und Belastungszuständen untersucht.

\section{Einblicke aus experimentellen Untersuchungen}

Kurz nachdem die ersten Druckversuche im Nanoindenter an Säulen vorgenommen wurden, sind diese verformten Proben auch im TEM untersucht worden. Dazu sind die gedrückten Säulen mit Hilfe eines "Fokussierter Ionenstrahl Mikroskopes"(FIB) auf Elektronentransparenz nachgedünnt worden [7]. Somit lässt sich die Defektstruktur post mortem untersuchen. Abbildung 1.3 zeigt eine Abbildung von verformten Säulen im TEM und deren Mikrostruktur. Zu erkennen ist auf den Aufnahmen, dass lediglich 


\section{EINLEITUNG}
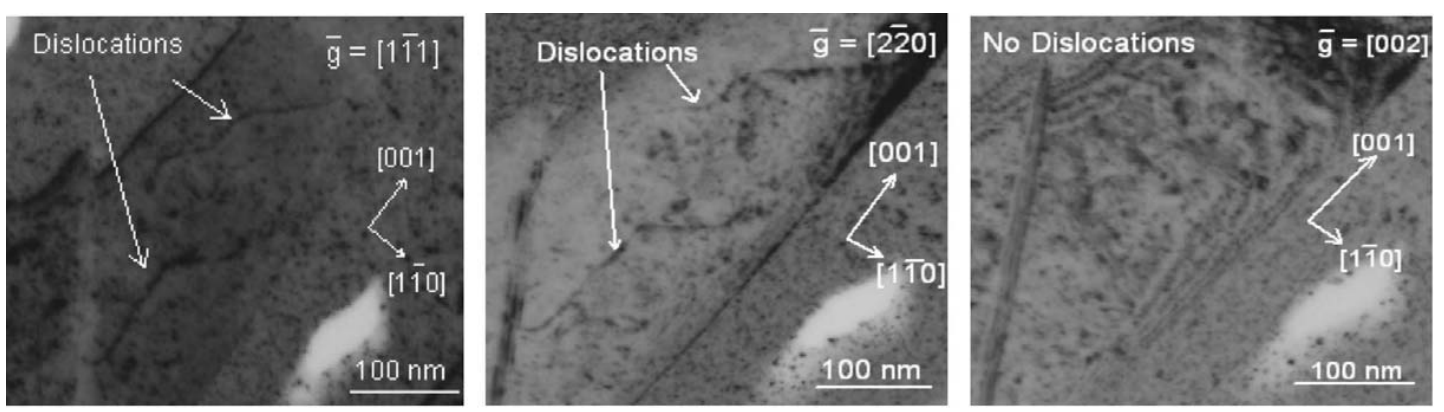

Abbildung 1.3: TEM Aufnahmen von verformten Gold-Säulen - Invertierte TEM Dunkelfeldaufnahmen einer verformten Gold-Säule unter verschiedenen Abbildbedingungen. Zu erkennen sind Versetzungssegmente, auf die in der gewählten Belastungsrichtung keine Kraft wirkt. (entnommen aus [7])

zwei Versetzungslinien in der Probe enthalten waren, die in einem Druckversuch entlang der [001]-Richtung keine Scherkraft spüren. Die Interpretation solcher Ergebnisse liefert also eine Bestätigung für die Vermutung, dass mobile Versetzungen in kleinen Proben diese an den freien Oberflächen verlassen. Damit ist eine weitergehende plastische Verformung nur dann möglich, wenn neue Versetzungen gebildet werden. Gerade aber dieser fundamentale Aspekt der Verformung ist nicht direkt mit Hilfe dieser Art von Experimenten zu untersuchen.

Eine sehr direkte Möglichkeit dieses zu tun ist folglich, Proben während des Experimentes im TEM zu verformen und dabei zu beobachten. Dieser Ansatz wurde in einigen Experimenten verfolgt $[8,9]$. Oh et al. präsentierten einen Ansatz [10], bei dem der Einfluss der Mikrostruktur der Probe weitestgehend ausgeschlossen werden sollte. Dazu wurde in einem speziellen Verfahren eine einkristalline Probe auf einem verformbaren Substrat präpariert. In diesem Aufbau konnte sogar gezielt der Größeneinfluss untersucht werden, indem unterschiedliche Schichtdicken hergestellt wurden. Aus den Ergebnissen lies sich ein Wechsel in dem dominierenden Verformungsmechanismus ableiten. In dicken Schichten wurden volle Versetzungen beobachtet, während in dünnen Schichten Stapelfehler aufgrund der Bewegung von Partialversetzungen auftraten. Abbildung 1.4 zeigt einige Beispiel dieser Arbeit. Das entwickelte Modell für die Erklärung des Wechsels in der Versetzungsart liefert einen Größenwert, der allerdings etwa eine Größenordnung unter dem experimentell bestimmten lag. Diese Art von Untersuchungen zeigte deutlich, dass die experimentelle Bewegungsrichtung die richtige war, die Beschreibungen in einem Modell aber noch einer Fortentwicklung bedurften. 


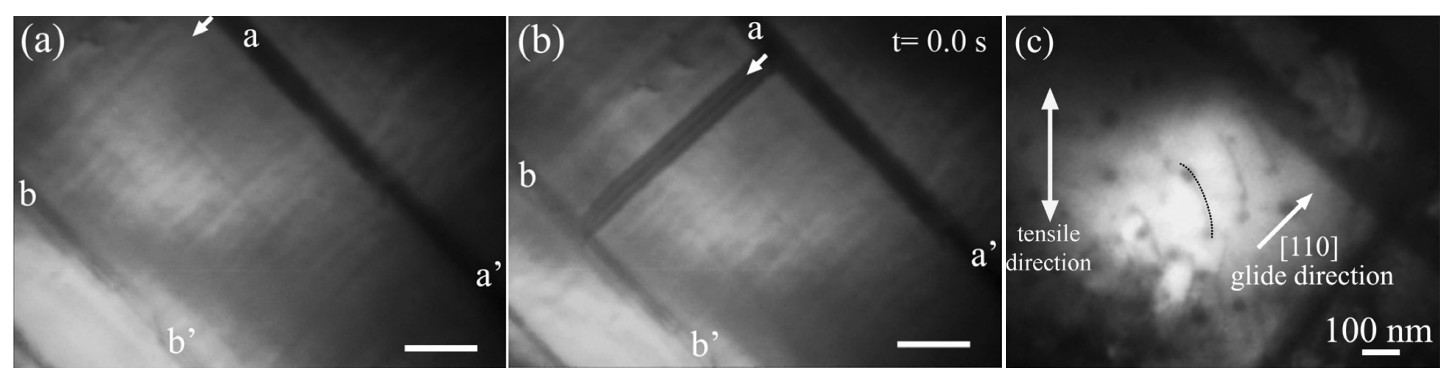

Abbildung 1.4: Wechsel des Verformungsmechanismus mit der Schichtdicke TEM Hellfeldaufnahmen von unterschiedlich dicken Gold Schichten auf Polyimid. a) und b) zeigen das Auftreten von Stapelfehlern als Produkt der Bewegung einer Partialversetung in $40 \mathrm{~nm}$ dicken Schicht. c) zeigt volle Versetzungen in einer verformten $160 \mathrm{~nm}$ Schicht. Die Bilder sind entnommen aus [10].

Bei der oben aufgeführten Herangehensweise muss allerdings immer auch der Einfluss des Substrates mit berücksichtigt werden. Durch das Aufwachsen auf einem Trägerfilm kommt es zu Spannungen, die durch Versetzungen oder Zwillingsbildung abgebaut werden müssen [11]. Diese Spannungsbeiträge der Grenzflächen überlagern sich mit denen, der extern aufgebrachten Spannung durch die Verformung im Experiment. Eine Entkopplung der Beiträge ist nicht mehr einfach möglich. Auch ist bekannt, dass die Grenzfläche zum Substrat einen direkten Einfluss auf die Versetzungsbewegung haben kann $[12,13]$. Aus diesem Grund liefern Experimente an freistehenden Proben meist zuverlässigere Einblicke in die Verformungsmechanismen und die Versetzungsnukleation. Ein Beispiel für eine solche Probe ist nano-poröses Gold. Die Breite der Ligamente genannten Verbindungselementen zwischen den Poren dieser schwammartigen Struktur kann bis zu wenige Nanometer betragen. Verformungsversuche an diesem Material lieferten einen direkten Hinweis auf den Typ der aktiven Versetzung [14]. So konnte in einer post mortem Untersuchung gezeigt werden, dass in den verformten Proben mit einem Ligamentdurchmesser von 5 bis $10 \mathrm{~nm}$ ausschließlich Partialversetzungen im Volumen gespeichert waren. Volle Versetzungen konnten hingegen in dieser Arbeit nicht beobachtet werden. Auch konnten in anderen Arbeiten in den Ligamenten Zwillinge nachgewiesen werden [15], die vermutlich durch die korrelierte Bewegung von Partialversetzungen gebildet wurden. Auch wenn im Fall von nanoporösem Gold durch die Struktur an den jeweiligen Ligamenten komplexe Spannungszustände auftraten, konnte ein Einblick in die Verformung gewonnen werden. 


\section{EINLEITUNG}

Eine ähnliche Situation stellt sich dar, wenn man die Verformung von kleinen Goldkontakten betrachtet, die mitterweile zahlreich in der Literatur beschrieben werden. Der beobachtete Verformungsmodus ist wieder geprägt durch das Auftreten von Partialversetzungen und Zwillingen. Durch methodische Weiterentwicklungen konnten diese Phänomene auch schon in situ im TEM beobachtet werden [16, 17]. Abbildung 1.5 verdeutlicht die dabei ablaufenden atomaren Prozesse. Neben der Aktivierung von leading und trailing Partialversetzungen wurde auch der Mechanismus für die Zwillingsbildung direkt beobachtet. Ein Zwilling bildet sich demnach, wenn auf benachbarten Ebenen gleichartige Partialversetzungen aktiv sind. Allerdings konnte die wiederholte
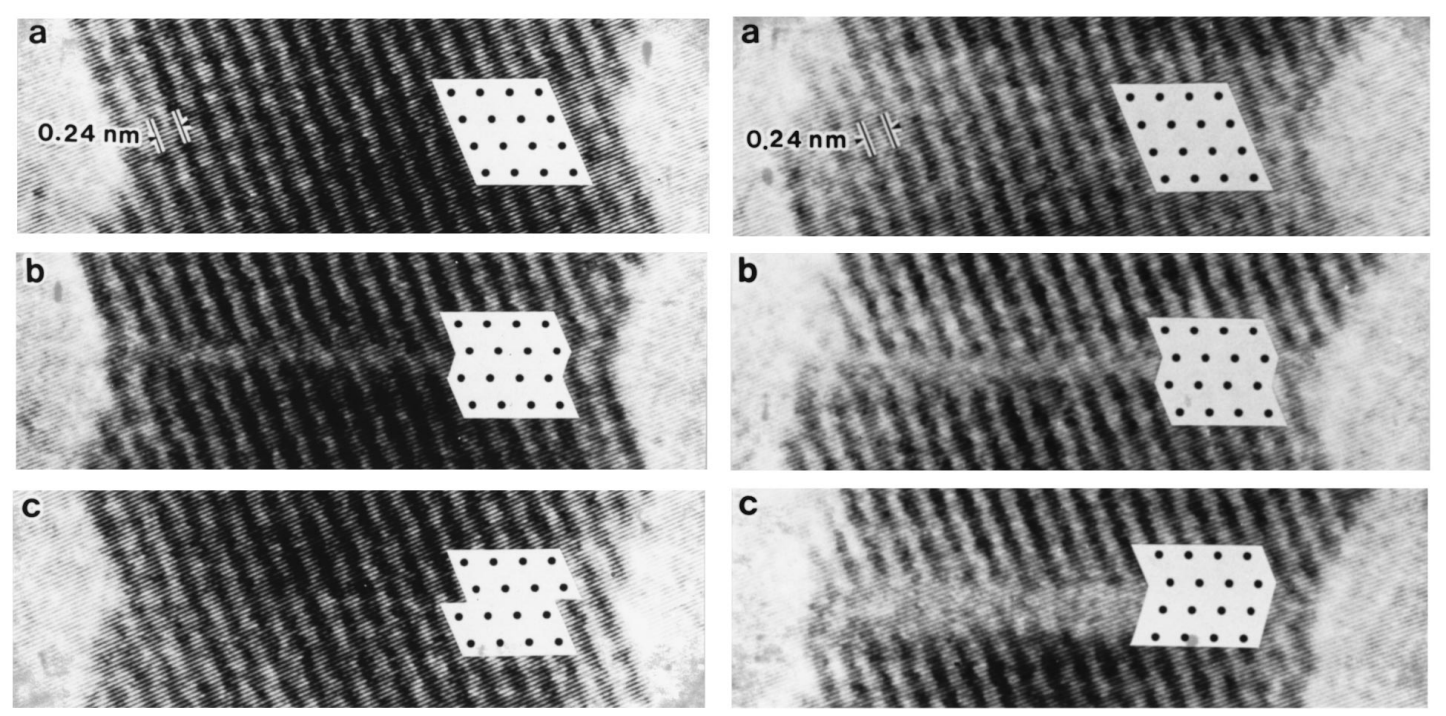

Abbildung 1.5: Atomar aufgelöste Verformung von Goldkontakten - Hochauflösende Elektronenmikroskopie zeigt die atomaren Prozesse, die mit der Verformung von Goldkontakten verknüpft ist. (aus [16])

Beobachtung dieses Verformungsmodus an freistehenden Proben bisher nicht genutzt werden, das Auftreten von Partialversetzungen stichhaltig mit gängigen Modellen zu erklären. Eine systematische Herangehensweise bietet hierbei die Modellierung dieser Systeme in molekulardynamischen (MD) Simulationen. Hier konnten einige Hinweise herausgearbeitet werden, die zum Verständnis der Verformung beitragen. 


\section{Einblicke durch MD Simulationen}

Eine systematische Untersuchung der Verformung von versetzungsfreien einkristallinen Gold-Nanodrähten mit unterschiedlichen Orientierungen wurde in [18] und [19] durchgeführt. Dort wurden Drähte modelliert, die sowohl in [111]-, [110]- als auch in [100]-Richtung belastet wurden. Für die Zugbelastung traten jeweils Partialversetzungen als charakteristische Defekte beim Beginn der Verformung auf. Ein Beispiel einer Simulation eines Zugversuches in [110]-Richtung ist in Abbildung 1.6 gezeigt. Bei der Verformung von [111]- und [100]-Drähten werden kurz nach der Nukleation der leading Partialversetzung auch die trailing Partialversetzungen gebildet. Dies lässt sich damit erklären, dass die Schmidfaktoren jeweils für die nachlaufende Partialversetzung höher sind [20].

Neben diesen geometrischen Vorhersagen konnte auch der Einfluss der Seitenfacetten

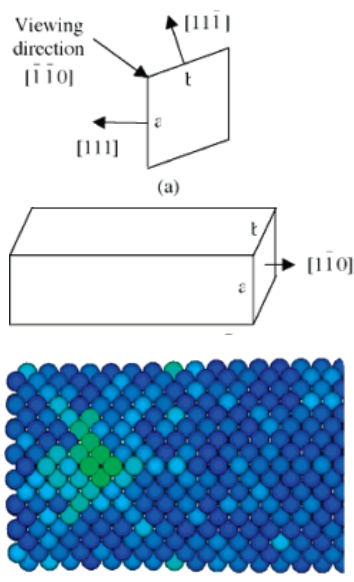

(b)

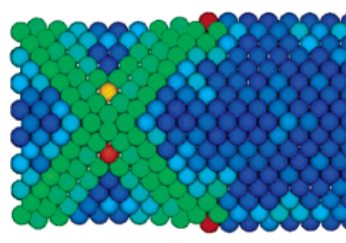

(c)

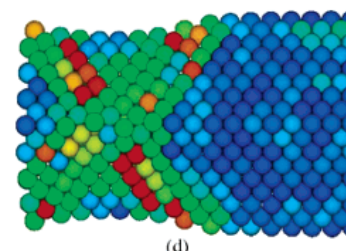

slipvec

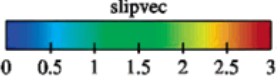

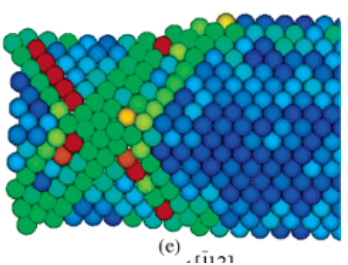

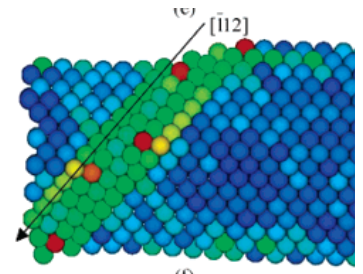

(I)

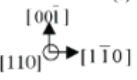

Abbildung 1.6: MD Simulation der Verformung eines Gold-Nanodrahtes - MD Simulation der Verformung eines [110]-Gold-Nanodrahtes. Man erkennt das Auftreten von Partialversetzungen auf zwei $\{111\}$-Ebenen, die den Draht komplett durchlaufen. (aus [19])

der Drähte untersucht [18] werden. Dabei stellte sich heraus, dass es bei Kupfer-Drähten mit [110]-Orientierung möglich ist, über die Aktivierung von Partialversetzungen auf benachbarten Ebenen einen geschlossenen Zwilling auf der gesamten Drahtlänge zu erreichen. Mit dieser Zwillingsbildung ist eine Reorientierung der Zugachse von [110] zu [100] verbunden. Dieses besondere Verhalten ist deshalb möglich, weil die ursprüngli- 


\section{EINLEITUNG}

chen $\{111\}$-Facetten durch die Zwillingsbildung in perfekte $\{100\}$-Facetten überführt werden können, die ebenfalls niedrige Oberflächenenergien haben. In [110] orientierten Drähten, bei denen die Oberflächen durch $\{110\}$-Facetten gebildet wurden, war dies nicht zu beobachten. Im Fall von Gold führt die niedrige Stapelfehlerenergie allerdings dazu, dass ein Zusammenwachsen der Zwillinge dadurch unterbunden wird, dass auf zwei gleichberechtigten Gleitebenen Stapelfehler entstehen. Diese können sich durchkreuzen, was dann ein ausreichendes Hindernis für die Zwillingsbildung darstellt.

Die Besonderheit bei MD Simulationen ist dabei sicherlich die Zeitskala, auf der die Verformung stattfindet. Aufgrund der begrenzten Systemgröße und Simulationsdauer ergeben sich durchweg hohe Dehnraten im Bereich von $10^{6} / \mathrm{s}$ bis $10^{8} / \mathrm{s}$ [21]. Die Auswirkungen auf den beobachteten Verformungsmechanismus werden jedoch nur selten kritisch diskutiert [22]. Die Ergebnisse der Simulationen sollten daher nicht direkt als Vorhersage für reale Experimente verstanden werden, sondern dafür genutzt werden Mechanismen daraus abzuleiten [23].

\subsubsection{Motivation der Arbeit}

Alle diese experimentellen Ergebnisse und Simulationen zeichnen ein recht einheitliches Bild der Verformung von nanoskaligen Goldproben. Demnach wird die Verformung in erster Näherung durch die Schmidfaktoren bestimmt. Das Auftreten von Stapelfehlern und die Zwillingsbildung konnten im Experiment bestätigt werden, wenn die Proben einige Nanometer klein waren. Daraus ergibt sich die Fragestellung, ob auch größere Proben, die eine homogene Spannung spüren, auf dieselbe Art verformen. Auch ergibt sich die Frage, ob es einen Übergang zu dem Verformungsverhalten von makroskopischen Proben gibt und was diesen Wechsel bedingt. Dort werden in der Regel keine Partialversetzungen beobachtet. Schlussendlich sollte mit dieser Arbeit versucht werden, die Versetzungsnukleation und deren wichtige Parameter direkt zu untersuchen und aus den Ergebnissen einen Beitrag zum Verständnis dieses Themenkomplexes zu liefern.

Ausgehend von den vorliegenden Arbeiten zur Untersuchung des Verformungsverhaltens von Gold lässt sich ein Experiment formulieren, das die verbleibenden Probleme angeht. Aus Stabilitätsgründen sollte ein Aufbau gewählt werden, der die nötige Kontrollierbarkeit während des gesamten Versuches garantiert. Dies ist am einfachsten 
damit zu erreichen, dass gänzlich auf die Messungen von Spannung oder Dehnung verzichtet wird. Somit lässt sich ein wirklich dehnungskontrollierter Zugversuch realisieren. Die verwendeten Proben müssen freistehend sein und keine mikrostrukturellen Defekte aufweisen, damit die Versetzungsnukleation aus dem ungestörten Material heraus untersucht werden kann. Somit steht auch von vornherein eine Präparation der Zugprobe mit dem FIB nicht zur Diskussion. Für die Untersuchung der Größenabhängigkeit sind im TEM Proben bis etwa $300 \mathrm{~nm}$ Dicke geeignet. Ein Probentransfer ist derzeit bis zu einer Größe von $40 \mathrm{~nm}$ realisierbar [24]. Diese Grenzwerte bestimmen somit den Größenbereich der zu untersuchenden Proben.

Um eine vollständige Charakterisierung der mechanischen Eigenschaften der Proben zu erreichen, wurde die quantitative Untersuchung der Drähte in einer eigenständigen Arbeit geleistet. In [24] wurden dieselben Drähte im Rasterelektronenmikroskop (SEM) getestet und Spannung-Dehnung-Kurven aufgenommen. In post-mortem Untersuchungen wurde im TEM die sich ergebende Defektstruktur analysiert. Es hat sich dabei gezeigt, dass die auftretenden Defekte soweit vergleichbar sind, dass die beiden Arbeiten komplementär sind. Damit ist sichergestellt, dass im weiteren Verlauf an geeigneter Stelle Erkenntnisse aus der Arbeit [24] mit in die Beurteilung der Ergebnisse hier einfließen können.

In kürzlich veröffentlichen Arbeiten sind zwei weitere Varianten der oben skizzierten Herangehensweise vorgestellt worden. Die Gruppe um Jong-Hyun Seo untersuchte [110] orientierte Gold-Drähte in einem Zugaufbau, der für den Einsatz im SEM geeignet ist [25]. Dieser besteht aus einem Mikromanipulator, der verwendet wird, um die einkristallinen Proben uniaxial zu ziehen. Die verwendete Dehnrate betrug etwa $4 \cdot 10^{-2}$. Durch TEM Untersuchungen konnte an getesteten Drähten der Verformungsmechanismus aufgeklärt werden. In den verwendeten Proben kam es demnach durch die Verformung zu einer Verzwillingung auf der Gesamtlänge des Drahtes. Dieses Zwillingswachstum setzte nach einer anfänglich elastischen Dehnung ab einer Spannung von 1,54 GPa ein. Die weitergehende Verformung bis etwa 50\% Dehnung verlief auf sehr viel niedrigerem Spannungsniveau. Eine Erklärung für den beobachteten Verlauf wird nicht geliefert und auch der Spannungszustand und die Stabilität des Systems zeigen, dass es in dem gewählten Aufbau nicht unbedingt jederzeit möglich war die Verformung zu kontrollieren. 


\section{EINLEITUNG}

Eine weitere Arbeit einer Gruppe aus Karlsruhe [26] untersuchte ebenfalls die Verformung von [110]-orientierten Gold-Drähten im REM mit Hilfe eines modifizierten Nanoindenters. Mit diesem Aufbau können während der Verformung direkt Kraft-WegKurven aufgenommen werden. Die dabei gewonnenen Kurven sind für eine Klasse von Drähten äquivalent zu den ermittelten Kurven aus der Diplomarbeit von Bahne Kapelle [24]. Neben diesem Verhalten wird auch noch eine zweite Klasse identifiziert, deren Verformung wiederum durch das Ausbilden eines großen Zwillings gekennzeichnet wird. Aus der Kombination dieser experimentellen Ergebnisse mit weiteren MD Simulationen wird vermutet, dass der Unterschied zwischen den beiden Klassen durch eine kritische Lamellendicke bestimmt wird. Diese führt dazu, dass die durch die Formänderung bedingten Spannungskonzentrationen eine selbststimulierende Wirkung auf die Bildung von weiteren zwillingsbildenden Partialversetzungen hat. Wird die kritische Größe nicht erreicht, wird die Verformung von unabhängigen Nukleationsprozessen bestimmt.

Die in dieser Arbeit vorgestellten Ergebnisse unterscheiden sich dadurch von den vorgestellten Arbeiten, dass gänzlich auf die Messung von Spannung und Dehnung verzichtet wird. Dadurch kann, wie oben erwähnt, ein wirklich dehnungskontrollierter Zugversuch aufgebaut werden [27]. Dies ermöglicht zu jeder Zeit die volle Kontrolle über die Verformung. Damit sind auch statistische Auswertung der Nukleationsprozesse direkt während des Versuches möglich. Auf den folgenden Seiten wird nach einer kurzen theoretischen Einführung der gewählte Aufbau beschrieben und die Ergebnisse der Experimente dargestellt. In der Diskussion dieser Ergebnisse wird darüber hinaus ein Modell präsentiert, dass die Versetzungsnukleation auf atomarer Skala beschreibt und eine sehr guten Übereinstimmung mit den Ergebnissen liefert. Mögliche Erweiterungen der Arbeit werden im letzten Kapitel aufgezeigt. 


\section{2}

\section{Theoretische Grundlagen}

In diesem Kapitel sollen die Grundlagen dargelegt werden, die nötig sind, um die gewonnenen Ergebniss in den folgenden Kapiteln beschreiben zu können. Im Wesentlichen wird hier auf die kristallographischen Besonderheiten von Gold und dessen Verformung sowie die Elektronenmikroskopie eingegangen. Im ersten Teil werden kurz die wichtigsten und relevanten Eigenschaften von fcc Materialien wiederholt. Dieser Abschnitt wird vielfach in verschiedensten Büchern diskutiert und stellt somit nur eine allgemeine Wiederholung dar. Im zweiten Teil sollen die mathematisch physikalischen Grundkonzepte der Transmissionselektronenmikroskopie vorgestellt und diese auf relevante Defekte angewendet werden. Das Ziel dabei ist es, das charakteristische Aussehen und Zustandekommen von Kontrasten in metallischen fcc Proben zu erläutern. Ausgegangen wird hierbei jeweils von der Howie-Whelan Gleichung, die dort eingeführt werden wird. Die Darstellung der relevanten Formeln folgt dabei den Ausführungen aus [28]. Aufgrund der Komplexität der Kontrastmechanismen und der zugrundeliegenden Theorie wurden die Ausführungen allerdings stark gekürzt und es werden nur die wirklich zum Verständnis nötigen Sachverhalte und deren Formeln erläutert.

\subsection{Kristallographie und Verformung}

Gold liegt bei Raumtemperatur in einer fcc Gitterstruktur vor. In dieser Struktur gibt es einige Richtungen und Ebenen, denen eine besondere Bedeutung zukommt. Insbesondere wenn die Probe aufgrund einer angelegten Spannung ihre Form dauerhaft verändern soll, werden diese Nebenbedingungen sichtbar. Um diese Einschränkungen und Beson- 


\section{THEORETISCHE GRUNDLAGEN}

derheiten verstehen zu können, ist in Abbildung 2.1 schematisch eine fcc Einheitszelle gezeigt.

Die erste Besonderheit, die in Abbildung $2.1 \mathrm{im}$ mittleren Bild dargestellt ist, sind die
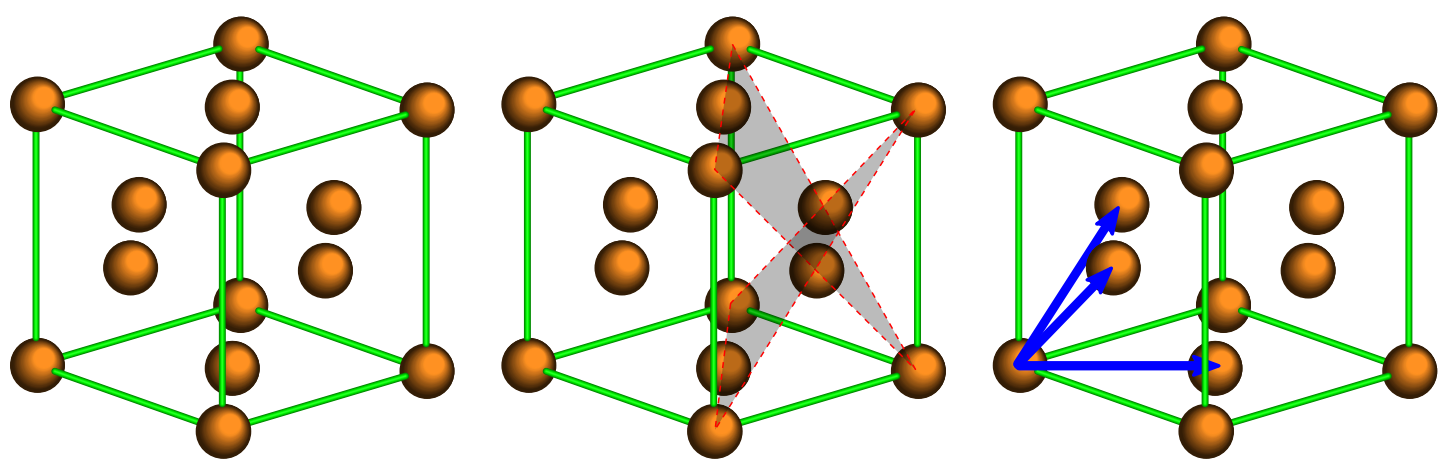

Abbildung 2.1: Schemazeichnung der fcc Einheitszelle - Auf den Seitenflächen des Würfels befindet sich jeweils ein weiteres Atom. Im mittleren Bild sind zwei $\{111\}$-Ebenen eingezeichnet, im rechten Bild drei äquivalente $<110>$-Richtungen.

$\{111\}$-Ebenen. Sie sind die Ebenen mit der höchsten Packungsdichte. Darüber hinaus ist der Abstand zwischen zwei benachbarten Ebenen dieses Types der größtmögliche innerhalb der fcc Struktur. Bei Gold beträgt der Abstand

$$
d_{111}=\frac{4,08 \AA}{\sqrt{1^{2}+1^{2}+1^{2}}}=2,36 \AA
$$

Als zweite relevante Größe für die Verformung stellen sich ausgezeichnete Richtungen heraus. Für die Bewegung der Atome ist gerade der kleinstmögliche Abstand von besonderem Interesse. Für den Fall des fcc Gitters ist dies in Abbildung 2.1 ganz rechts dargestellt. Von einem beliebigen Atom aus gesehen ist der nächste Nachbar in $1 / 2<110>-$ Richtung gelegen.

Tatsächlich findet man bei verformten fcc Kristallen genau diese Kombination aus Richtung und Ebene, die den maßgeblichen Einfluss haben. Die genaue Beschreibung der Verformung und die dabei beteiligten kristallographischen Richtungen werden im folgenden Abschnitt erläutert.

\subsubsection{Versetzungsnukleation und Versetzungstypen}

Für die plastische Verformung in Metallen ist im Allgemeinen die Bewegung von Versetzungen im Material notwendig. Überlegt man sich, welches Potential eine vorhandene 
Versetzung in einem sonst defektfreien Material spürt, ist dies das periodische Peierls Potential, das durch Atome im Gitter gebildet wird. Dementsprechend muss für diesen Fall auch nur diese Potentialbarriere überwunden werden, um eine Versetzung zu bewegen. Die Spannung, die dazu nötig ist, wird als Peierls-Spannung bezeichnet und lässt sich berechnen mit der Formel [29]

$$
\tau_{P}=\frac{2 G}{1-\nu} \exp \left(-\frac{2 \pi}{(1-\nu)} \frac{d}{b}\right)
$$

An dieser Formulierung wird deutlich, was phänomenologisch schon im vorherigen Abschnitt beschrieben wurde. Den geringsten Widerstand verspürt eine Versetzung auf einem Gleitsystem, wo der Abstand d zwischen den Ebenen möglichst groß ist und die einzelne Sprungweite b möglichst klein. Berechnet man die Spannung für den Fall von Gold erhält man einen sehr kleinen Wert von etwa $10 \mathrm{MPa}$. An realen Proben werden je nach Mikrostruktur aber deutlich höhere Werte gemessen. Der Grund dafür ist, dass das Konzept, wie es oben angenommen wurde, in realen Kristallen schnell an seine Grenzen stößt. Es ist zum Beispiel in makroskopischen Proben sehr selten, dass nur eine einzelne Versetzung in einem sonst ungestörten Gitter vorhanden ist. Die Wechselwirkung zwischen zwei Versetzungen wird mit Gleichung 2.2 aber nicht berücksichtigt. Darüber hinaus ist es in Proben, in denen die mögliche Laufweite sehr klein wird (d.h. wenige Nanometer) zum Beispiel realisierbar, dass eine vorhandene Versetzung die Probe ohne Wechselwirkung an einer freien Oberfläche verlässt. Weitere Verformung kann dann nur über neu gebildete Versetzungen erfolgen. Dieser Umstand wird jedoch ebenfalls in Gleichung 2.2 nicht erfasst.

Der Prozess der Versetzungnukleation ist sehr komplex und bis heute ist ein genaues atomistisches Bild für die beginnende plastische Verformung nicht verfügbar. So gibt es in der Literatur verschiedene Ansätze, die aufkeimende Verformung zu beschreiben. Da es sich im Verlaufe der Arbeit gezeigt hat, dass die Versetzungsnukleation auch für die Verformung von Gold-Nanodrähten einen entscheidenden Einfluss hat, wird die Dikussion der verfügbaren Modelle detailiert in Kapitel 6 vorgenommen.

Von besonderer Relevanz für diese Arbeit ist neben der Versetzungsnukleation auch die Frage nach der Art der Versetzung. Betrachtet man die Verzerrungsenergie, die mit einem Versetzungssegment der Länge L einer Stufenversetzung in einem Material verknüpft ist, gelangt man zu folgendem Ausdruck:

$$
E_{e l}=L \cdot \frac{G b^{2}}{4 \pi(1-\nu)} \ln \frac{R_{0}}{r_{0}} \approx L \cdot \frac{1}{2} G b^{2}
$$




\section{THEORETISCHE GRUNDLAGEN}

Hier bezeichnen $\mathrm{R}_{0}$ und $\mathrm{r}_{0}$ den äußeren bzw. inneren Abschneideradius, bis wohin man das Verzerrungsfeld betrachtet. Die weiteren Parameter sind materialabhängig, wie das Schermodul $G$, die Poissonzahl $\nu$ und der Burgersvektor $b$. An dieser Formulierung ist erkennbar, dass die Größe des Burgersvektors quadratisch in die Verzerrungsenergie eingeht und damit diese im Wesentlichen bestimmt. Nach den Ausführungen in 2.1 sind die Gleitrichtungen in fcc durch den kleinsten Abstand vorgegeben. Schaut man sich allerdings eine $\{111\}$-Ebene in der Schemazeichnung an, wird eine weitere Möglichkeit der Verschiebung erkennbar (vgl. Abbildung 2.2). In dieser Zeichnung ist dargestellt,

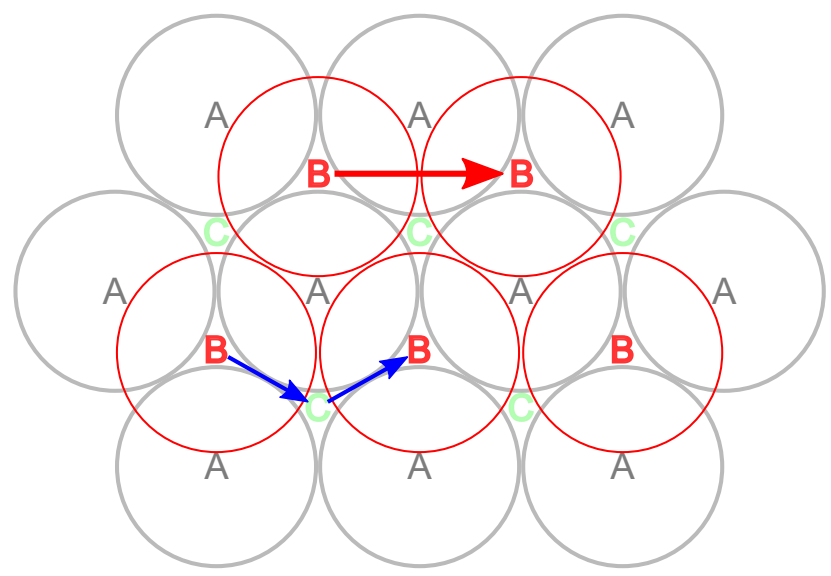

Abbildung 2.2: Schemazeichnung einer $\{111\}$-Ebene in einem fcc Gitter - Neben der vollen Versetzung in $<110>$-Richtung ist es auch möglich, zwei Versetzungen in $<112>$ Richtungen zu bilden. Dies verringert die elastische Verzerrungsenergie, führt aber zu einem Stapelfehler zwischen den Partialversetzungen

wie eine Versetzung in $<110>$-Richtung die Atompositionen verändern würde. Man sieht, dass nach der Versetzungsbewegung das Gitter ungestört erhalten bleibt. Eine zweite Möglichkeit der Versetzungsbewegung ist zusätzlich eingezeichnet. Diese beinhaltet die Bewegung von zwei $<112>$-Versetzungen, die in der Summe auch zu einer vollen Bewegung in $<110>$-Richtung führen. Diese Konfiguration verringert die elastische Verzerrungsenergie, da die Summe der beiden Beiträge der Partialversetzungen kleiner ist als die Verzerrungsenergie einer vollen Versetzung.

$$
2 G \cdot(a / 6<112>)^{2}=\frac{G a^{2}}{3}<\frac{G a^{2}}{2}=G \cdot(a / 2<110>)
$$

Allerdings führt die Aufspaltung in zwei Partialversetzungen dazu, dass nach der ersten Bewegung die Atome nicht auf einer fcc Gitterposition landen. In Abbildung 2.2 
bedeutet dies, dass B-Atome erst auf einer C-Position enden bevor sie sich durch die zweite Versetzung entgültig wieder auf einer B-Position befinden. Die Stapelfolge zwischen diesen beiden Einzelversetzungen lautet demnach kurzfristig C-A-C, was einer hcp-Stapelfolge entspricht. Diese vom Material unvorgesehene Konfiguration ist mit einer erhöhten Energie verbunden. Diese Stapelfehlerenergie $\gamma$ ist materialabhängig und beträgt bei Gold etwa $40 \mathrm{~mJ} / \mathrm{m}^{2}$ [30]. Im realen Kristall herrscht daher ein konkurrierendes Verhalten vor: Zum Einen verringert man die Gesamtenergie durch die Aufspaltung in zwei Partialversetzungen, zum Anderen muss Energie aufgewendet werden, da ein Stapelfehler gebildet wird. Nimmt man alle diese wechselwirkenden Terme bei zwei sich auf der gleichen Gleitebene befindlichen Versetzungen zusammen [31], kann eine Gleichgewichtsaufspaltungsweite berechnet werden. Diese Konfiguration wird im spannungsfreien Zustand allein aufgrund der oben beschriebenen Energiebilanz eingenommen.

$$
d_{0}=\frac{G b_{p}^{2}}{4 \pi \gamma(1-\nu)}
$$

Für den Fall Gold liegen also alle vorhandenen Versetzungen als Paar von Partialversetzungen vor, deren Abstand etwa 2,5 nm beträgt. Die Auswirkungen einer außen angelegten Spannung auf das Verhalten der beiden Partialversetzungen und daraus folgende Implikationen für die größenabhängige Verformung vom Kristall wird im Kapitel 6 eingehend dargestellt.

Neben der kurzfristigen hcp-Koordination gibt es eine weitere Möglichkeit der Atombewegung auf einer $\{111\}$-Ebene. Durch Vertauschung der Reihenfolge der beiden Partialversetzungen in Abbildung 2.2 ist auch eine Nettobewegung in $<110>$-Richtung möglich. Allerdings ist die temporäre Konfiguration nach der Bewegung der ersten Partialversetzung eine C-A-A Stapelfolge. Die mit dieser Stapelreihenfolge verknüpfte Stapelfehlerenergie ist von derart hoher Energie, dass sie nie eingenommen wird. Somit wird deutlich, dass die Reihenfolge der Partialversetzungen bei der Vorhersage einer Verformung beachtet werden muss.

In fcc gibt es zwei verschiedene Arten von Stapelfehlern, die in Abbildung 2.3 schematisch dargestellt sind. Läuft eine Partialversetzung durch den Kristall wie oben beschrieben wurde, erhält man als Resultat einen intrinsischen Stapelfehler. Die Stapelfolge ist daher vom Typ A B C B C A B C. Hier wurde quasi eine Position in der Reihenfolge übersprungen und fehlt. Die zweite denkbare Möglichkeit ist, dass eine zusätzliche Postion eingeführt wird, es ergibt sich A B C B A B C. Die Realisiserung 

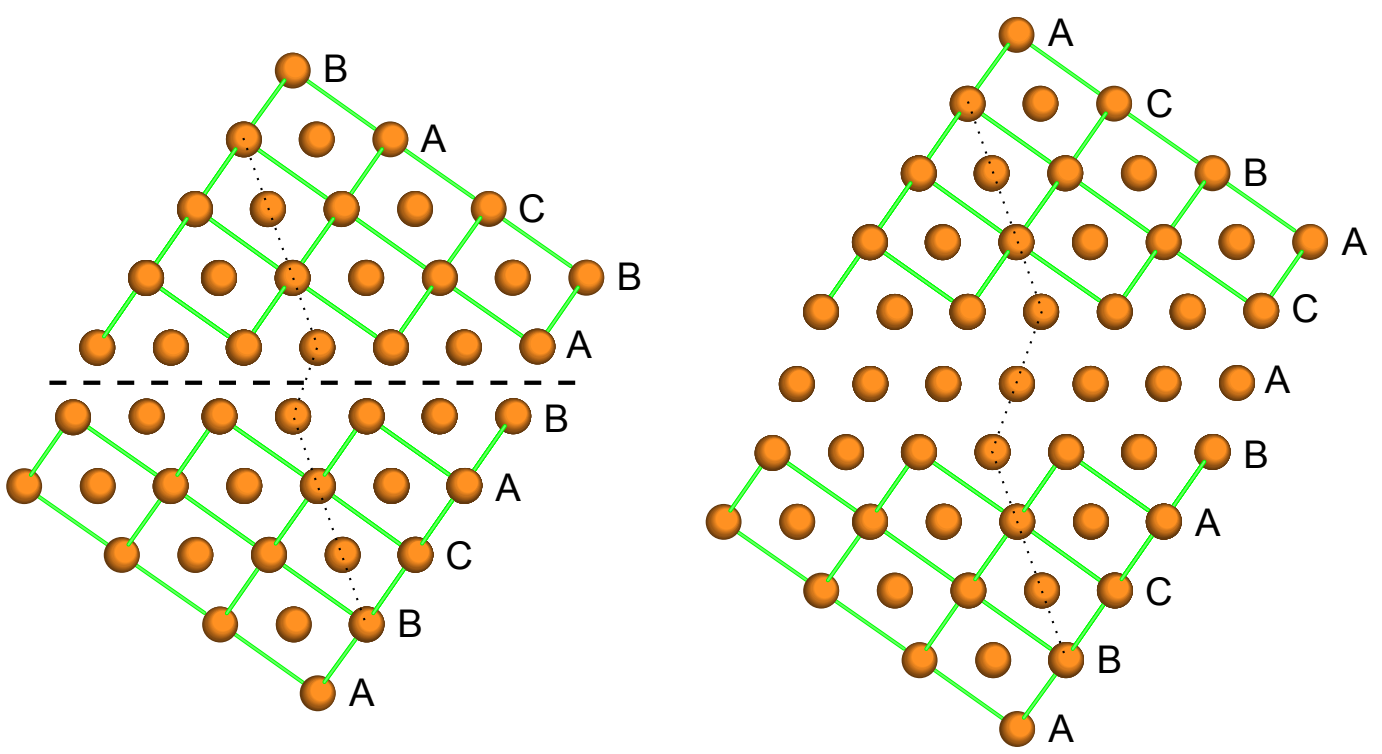

Abbildung 2.3: Typen von Stapelfehlern in fcc - Zeichnung der Atompositionen im fcc Gitter für einen intrinisischen (links) und einen extrinsischen (rechts) Stapelfehler. Die Stapelfolge entspricht einem Kristall, bei dem eine $\{111\}$-Ebene herausgenommen (intrinsisch) oder zusätzlich eingeführt (extrinsisch) wurde.

eines solchen Stapelfehlers ist nur über einen komplexeren Vorgang möglich, dieser geht vom intrinischen Stapelfehler aus. Läuft auf der nächsten $\{111\}$-Ebene eine gleichartige $1 / 6<112>$ Partialversetzung durch den Kristall, wird ein extrinsischer Stapelfehler gebildet.

\subsubsection{Das Schmidsche Schubspannungsgesetz}

Aufgrund der Einschränkung der Verformungsrichtung im fcc Gitter ist zum Beispiel bei uniaxialen Belastungen entscheidend, wie die Gleitsysteme im Kristall relativ zur Belastungsrichtung orientiert sind. Dabei sind zwei kristallografische Größen bei der Beschreibung relevant. Zum Einen findet Abgleitung auf $\{111\}$-Ebenen statt, zum Anderen ist die Richtung entlang $<110>$ oder $<112>$ vorgegeben. Diese geometrischen Beziehungen sind in Abbildung 2.4 dargestellt. Mit Hilfe dieser Überlegung ist es nun möglich jede Zug- oder Druckspannung $\sigma$ bei bekannter Belastungsrichtung in die entsprechend im Gleitsystem wirkende Scherspannung umzurechnen. Die entsprechende Formel lautet

$$
\tau=\sigma \cos \kappa \cos \lambda
$$




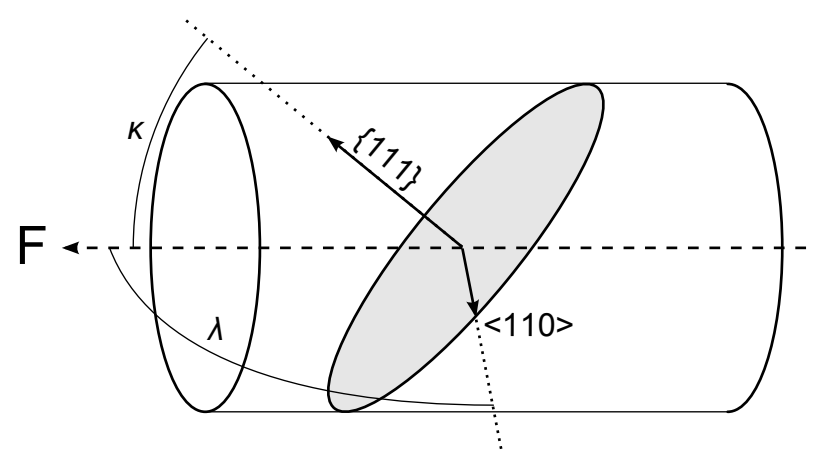

Abbildung 2.4: Skizze zur Veranschaulichung des Schmidfaktors - Die Winkel $\lambda$ und $\kappa$, die die Lage der Gleitrichtung bzw. Gleitebene relativ zu Belastungsrichtung bezeichnen, bestimmen wie groß die wirkende Scherspannung auf dem Gleitsystem ist.

Dabei fasst man die beiden trigonometrischen Faktoren als sogenannten Schmidfaktor $\mathrm{m}_{S}$ zusammen

$$
m_{S}=\cos \kappa \cos \lambda
$$

Eine Grenzfallbetrachtung zeigt, dass dieser Faktor nur Werte zwischen 0 und 0,5 annehmen kann. Eine Zugspannung wirkt also dann am stärksten auf einem Gleitsystem, wenn sowohl die Ebene als auch die Gleitrichtung im Winkel von $45^{\circ}$ zur Belastungsrichtung stehen. In diesem Fall beträgt

$$
\tau=0,5 \cdot \sigma
$$

\subsubsection{Mechanismen der Zwillingsbildung}

Neben der oben aufgeführten Möglichkeit eines Kristalls bei Anlegen einer externen Spannung, seine Form durch die Bildung und Bewegung von Versetzungen zu verändern, gibt es einen anderen für diese Arbeit relevanten Mechanismus, die Verformung durch Zwillingsbildung. In diesem Abschnitt soll allerdings nicht auf die Zwillingsbildung im Allgemeinen eingegangen werden, sondern vielmehr die für eine fcc Gitterstruktur wichtigen Prinzipien aufgezeigt werden.

Als Zwilling bezeichnet man einen Teil des Kristalls, der sich in eine spiegelsymmetrische Lage gegenüber dem Rest des Kristalls befindet. Beschrieben wird ein Zwilling durch die Ebene, an der der Kristall gespiegelt wurde und durch die Richtung, in welche in dieser Ebene die Atome verschoben wurden. In fcc sind die dabei beteiligten Ebenen und Richtungen über die Gitterstruktur vorgegeben. Man findet, dass Zwillinge 


\section{THEORETISCHE GRUNDLAGEN}

$\{111\}$-Ebenen als Spiegelebenen haben und die Scherung in $<112>$-Richtung erfolgt. Abbildung 2.5 zeigt diese Geometrie. Die Bildung eines Zwillings während der Ver-

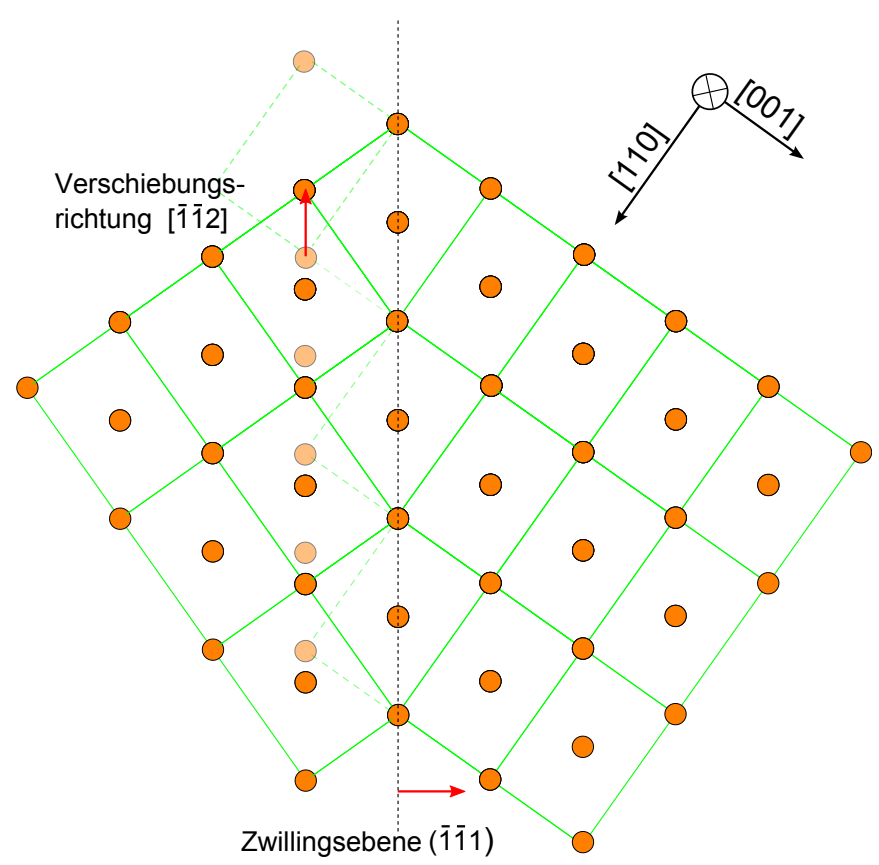

Abbildung 2.5: Schematische Darstellung eines Zwillings in fcc - Die beiden Größen, die einen Zwillingssystem allgemein charakterisieren sind die Zwillingsebene und die Verschiebungsrichtung. In fcc handelt es sich dabei um eine $\{111\}$-Ebene und eine $<112>-$ Richtung

formung kann durch mehrere Mechanismen geschehen [32]. Die in der Literatur am häufigsten diskutierten sind dabei:

- der sog. „pole“-Mechanismus

- Martensitische Transformation

- Nukleation von Partialversetzungen auf benachbarten Ebenen.

In dieser Liste wird die martensitische Transformation nicht als Verformungszwilling angesehen, sondern als Transformationszwilling, daher wird dieser Teil hier nicht weiter betrachtet, da er für Gold nicht relevant ist. Der erste Punkt findet sich seit langer Zeit in der Literatur [33]. Ausgegangen wird in diesem Modell davon, dass mindestens ein festliegendes Versetzungselement als Hindernis für die Versetzungsbewegung auf einer schneidenden Ebene agiert. Um dieses Hindernis kann nun eine Ausbuchtung herum 
geschehen und ein Multiplikationsprozess einsetzen. Bewegt sich die Versetzung nach jedem Multiplikationsumlauf um eine Gleitebene weiter nach oben oder unten, entsteht ein Zwilling.

Da in den im Rahmen dieser Arbeit untersuchten Proben die Versetzungsdichte bei Beginn des Versuches gleich Null ist, scheidet dieser Vorgang allerdings als Mechanismus zur Zwillingsbildung aus. Die Aufenthaltsdauer der Versetzungen während der Verformung ist so kurz, dass zu keinem Zeitpunkt die Versetzungskonstellation für einen „pole“-Mechanismus beobachtet werden konnte. In einigen Drähten waren zwar Versetzungsegmente während der Verformung über einen längeren Zeitraum im Draht gespeichert, allerdings kam es in diesen Fällen nicht zu einer Zwillingsbildung. Aus diesen Gründen ist es nicht nötig, diesen Aspekt genauer zu beleuchten und im Detail zu erklären.

Der dritte Punkt in der obigen Aufzählung ähnelt dem „pole“-Mechanismus insofern, als dass auch in diesem Fall ein Wachstum des Zwillings auf benachbarten Ebenen betrachtet wird. Allerdings bedarf es hierbei keines Multiplikationsprozesses und somit auch keiner vorhandenen Versetzung. Der betrachtete Bildungsmechanismus führt die Idee des Stapelfehlers aus Abschnitt 2.1.1 fort. Dort wurde beschrieben wie erst ein intrinsischer und dann ein extrinsischer Stapelfehler gebildet werden kann. Nimmt man eine dritte Partialversetzung auf wiederum benachbarter $\{111\}$-Ebene an und lässt sie durch den Kristall laufen, wird ein Gebiet im Kristall geschaffen, dass in Spiegelsymmetrie zur Ausgangsorientierung steht. Das Schema dieses Verfahrens ist in Abbildung 2.6 gezeigt.

\subsubsection{Kristallographie der Zugversuche}

Durch den Transfer (der in Abschnitt 4.1 beschrieben wird) und insbesondere die [110]Wachstumsrichtung der Drähte (vgl. dazu auch Abschnitt 3.2) sind die möglichen Gleitsysteme, die während der Verformung aktiv sind, von vornherein festgelegt. Denkbar ist die Verformung durch volle Versetzungen und Partialversetzungen. Diese unterscheiden sich in den aufgelösten Scherspannungen durch die Lage der Gleitrichtung relativ zur Zugachse. Abbildung 2.7 zeigt beispielhaft eine mögliche Gleitebene mit allen auf dieser Ebene beteiligten Verschiebungsrichtungen. Die einzelnen Schmidfaktoren aller relevanten Gleitsysteme für einen Zugversuch in [110] Richtung sind in Tabelle 2.1 zusammengefasst. Aufgrund der senkrechten Orientierung der Ebenen (1111) und (i11) 


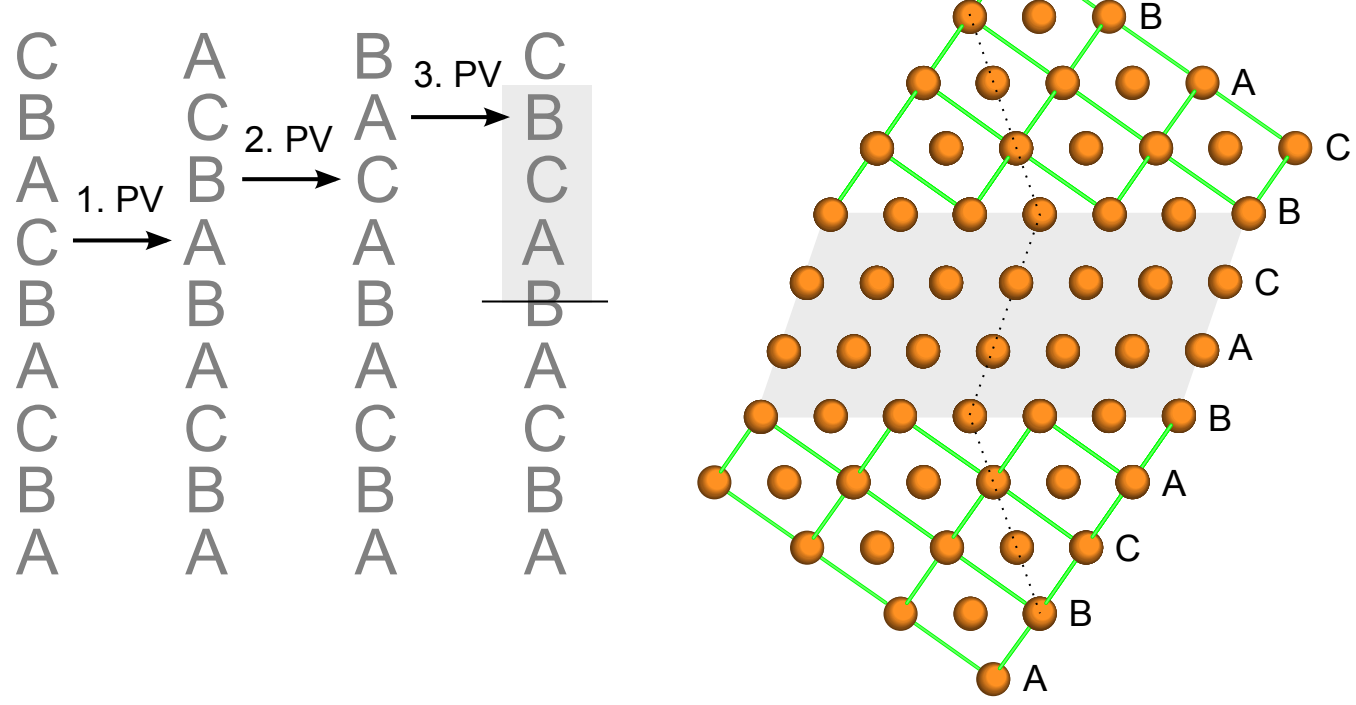

Abbildung 2.6: Schematische Zeichung der Zwillingsbildung - Durch das Aktivieren von Partialversetzungen auf benachbarten $\{111\}$-Ebenen ist die Bildung eines Zwillings im Kristall möglich. Dies ist mit Hilfe der Stapelreihenfolge und den zugehörigen Atompositionen in dieser Schemazeichnung dargestellt.

zur Zugachse wirkt dort keine Scherspannung und die Gleitsysteme sind nicht aktiv.

\subsection{Elektronenbeugung und reziproker Raum}

Elektronenbeugung als Methode für die Materialuntersuchung wird schon lange verwendet. Dabei ist die Tatsache, dass man überhaupt Beugungsphänomene auch mit Elektronen beobachten kann, einfach mit Hilfe der de Broglie Relation zu verstehen. Danach kann jedem Teilchen, das einen Impuls $p$ besitzt, auch eine Wellenlänge $\lambda$ zugeordnet werden. Die Konstante, die beiden Größen miteinander verbindet, ist das Plancksche Wirkungsquantum $h$.

$$
\lambda=\frac{h}{p}
$$

Im Falle der Elektronenmikroskopie ist der Impuls des Elektrons mit der Beschleunigungsspannung verknüpft. Dies bedeutet, dass auch die Wellenlänge mit der Spannung 


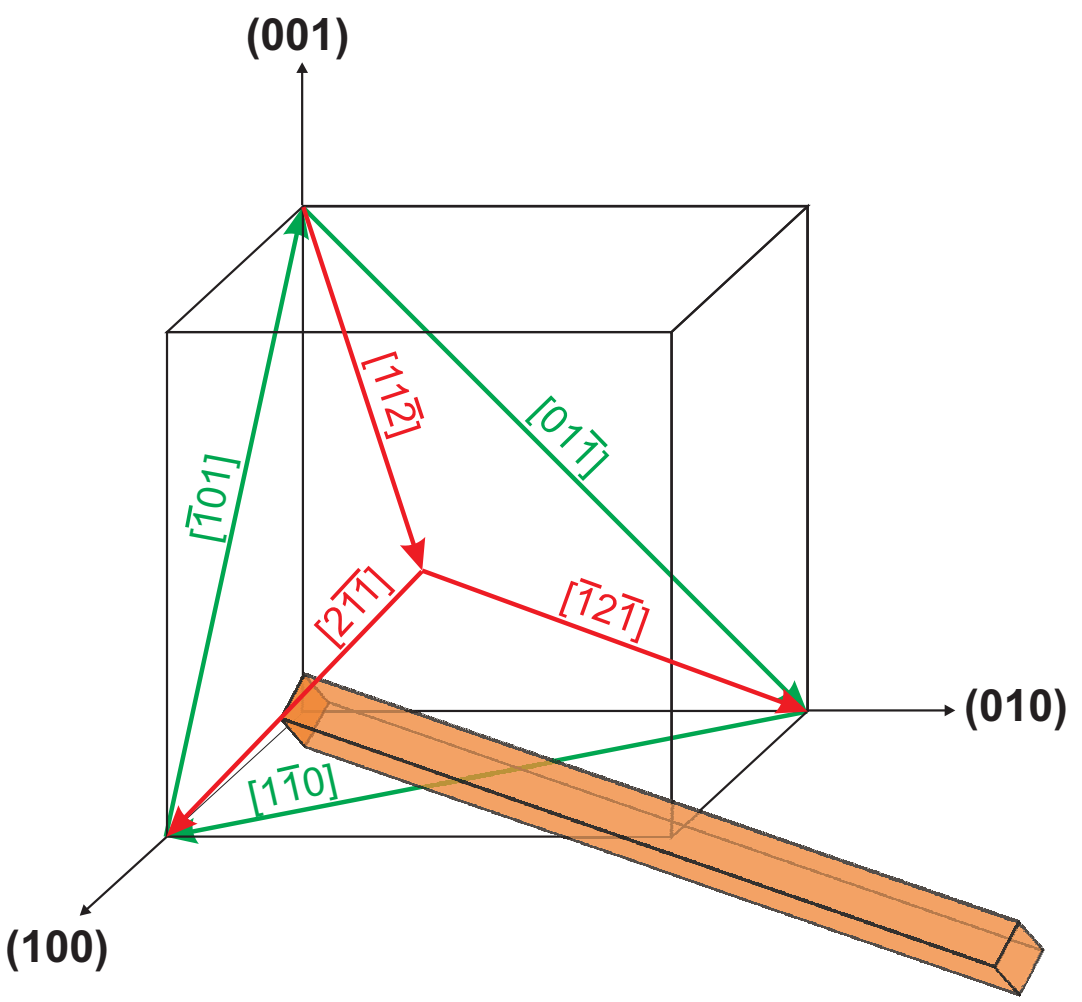

Abbildung 2.7: Kristallographie eines Zugversuches in [110]-Richtung - Zeichnerische Darstellung einer $\{111\}$-Gleitebene mit eingezeichneten vollen Versetzungen und Partialversetzungen

variiert werden kann. Betrachtet man zudem noch relativistische Effekte, die bei hohen Beschleunigugnsspannungen nicht mehr zu vernachlässigen sind, lässt sich die Wellenlänge ausdrücken über

$$
\lambda=\frac{h}{\left[2 m_{0} e E\left(1+\frac{e E}{2 m_{0} c^{2}}\right)\right]^{1 / 2}} .
$$

In dieser Gleichung bezeichnet $\mathrm{m}_{0}$ die Ruhemasse des Elektrons und $E$ die Beschleunigungsspannung des Mikroskops. Die Konstanten $e$ und $c$ stehen für die Elementarladung und die Vakuum-Lichtgeschwindigkeit. Mit dieser Formulierung ist dann auch im Realraum die Beugung zu verstehen. Diese kann im einfachsten Fall mit Hilfe des Bragg-Gesetzes beschrieben werden. Danach tritt konstruktive Interferenz an einem Gitter auf, wenn die Bedingung

$$
n \cdot \lambda=2 d \sin (\theta)
$$




\begin{tabular}{ccccc} 
Gleitebene & $\begin{array}{c}\text { Volle } \\
\text { Versetzung }\end{array}$ & Schmidfaktor & $\begin{array}{c}\text { Partial- } \\
\text { versetzung }\end{array}$ & Schmidfaktor \\
\hline \hline$(111)$ & {$[01 \overline{1}]$} & 0,408 & {$[11 \overline{2}]$} & 0,471 \\
& {$[1 \overline{1} 0]$} & 0 & {$[1 \overline{2} 1]$} & 0,236 \\
& {$[10 \overline{1}]$} & 0,408 & {$[\overline{2} 11]$} & 0,236 \\
\hline$(11 \overline{1})$ & {$[011]$} & 0,408 & {$[112]$} & 0,471 \\
& {$[1 \overline{1} 0]$} & 0 & {$[\overline{1} 21]$} & 0,236 \\
& {$[101]$} & 0,408 & {$[2 \overline{1} 1]$} & 0,236 \\
\hline \hline
\end{tabular}

Tabelle 2.1: Schmidfaktoren möglicher Gleitsysteme für einem Zugversuch in (110)Richtung

erfüllt ist. Dies bedeutet, dass alle von den Streuzentren ausgehenden Sekundärwellen gleicher Wellenlänge phasenrichtig in die betrachtete Richtung überlagert werden. In alle anderen Richtung tritt eine destruktive Interferenz auf und es wird keine Intensität beobachtet.

Die Darstellung eines beliebigen Vektors erfolgt im Realraum über drei Basisvektoren des Kristallgitters

$$
\vec{x}=n_{1} \vec{a}+n_{2} \vec{b}+n_{3} \vec{c}
$$

Allerdings ist es für die komplexeren Beugungsprobleme nicht immer vorteilhaft, diese über einen Formalismus im Realraum zu beschreiben. Aus diesem Grunde wird in der Elektronenmikroskopie eine äquivalente Beschreibung verwendet, diese bedient sich des reziproken Raumes. Der reziproke Raum stellt dabei im Wesentlichen die Fouriertransformierte des Realraumes dar. Danach stellt jeder Punkt im reziproken Raum also eine Frequenz von Abständen im Realraum dar. Die Basisvektoren $\mathbf{r}^{*}$ dieses Raumes lassen sich über die Vorschrift

$$
a^{*} \cdot b=a^{*} \cdot c=b^{*} \cdot c=b^{*} \cdot a=c^{*} \cdot b=c^{*} \cdot a=0
$$

ableiten. Damit lässt sich äquivalent zum Realraum auch jeder beliebige Vektor im reziproken Raum durch eine Linearkombination von Basisvektoren darstellen.

$$
\vec{K}=m_{1} \overrightarrow{a^{*}}+m_{2} \overrightarrow{b^{*}}+m_{3} \overrightarrow{c^{*}}
$$


Besondere Bedeutung bekommen bei dieser Formulierung spezielle Vektoren $\mathbf{K}$, bei denen die $\mathrm{m}$ Werte ganzzahlig sind. In diesem Fall repräsentiert der gebildete Vektor den Normalenvektor der beschriebenen Ebene im Realraum. Das Tripel der m Werte wird dann auch Millersche Indizies mit $h, k, l \in \mathbb{Z}$ genannt.

$$
g_{h k l}=h \overrightarrow{a^{*}}+k \overrightarrow{b^{*}}+l \overrightarrow{c^{*}}
$$

Der Abstand dieser Ebene vom Ursprung ist gekennzeichnet mit $\mathrm{d}_{h k l}$ und steht mit dem Betrage des reziproken Vektors über

$$
d_{h k l}=\frac{1}{\left|g_{h k l}\right|}
$$

in Beziehung. Die Beschreibung von Beugung erfolgt in diesem Bild dann nach einfachen geometrischen Überlegungen. Eine einfallende Welle hat den Vektor $\mathbf{k}_{I}$. Der Vektor der gebeugten Welle erhält die Bezeichnung $\mathbf{k}_{D}$. Für den Fall von elastischer Wechselwirkung wird keine Energie übertragen und die Beträge der beiden Wellenvektoren sind gleich.

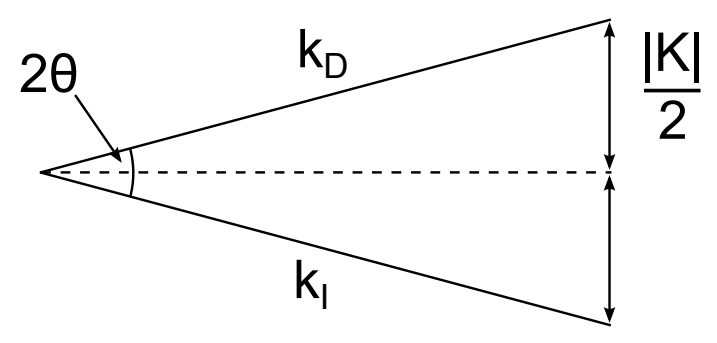

Abbildung 2.8: Geometrie der Beugung im reziproken Raum - Einfallende und gebeugte Welle haben betragsmäßig gleiche Wellenvektoren und schließen einen Winkel von $2 \theta$ ein

Die Bragg-Bedingung lässt sich dann mit Hilfe der oben hergeleiteten Beziehungen für das reziproke Gitter umschreiben:

$$
\frac{1}{d_{h k l}}=\frac{2 \sin \theta}{\lambda} .
$$

Benutzt man nun die Gleichung 2.16 und die geometrischen Überlegungen aus Abbildung 2.8 gelangt man zu der Laue Bedingung für Beugung:

$$
\vec{K}=\vec{g} \text {. }
$$




\section{THEORETISCHE GRUNDLAGEN}

Diese Beziehung besagt, dass dann konstruktive Interferenz auftritt, wenn der Differenzvektor aus einfallender und gebeugter Welle einem Vektor des reziproken Gitters entspricht.

Jedoch lassen sich in der Realität nicht nur Beugungsphänomene für die exakte Bedingung $K=g$ beobachten. Ein wichtiger Umstand ermöglicht Beugung auch für nicht genau eingehaltene Laue- bzw. Bragg-Bedingung: durch die Dicke der Probe sind die reziproken Gitterpunkte keine 0-dimensionalen Punkte mehr. In Elektroneneinstrahlrichtung beträgt die Probendicke meist nur wenige $100 \mathrm{~nm}$. Damit ist das reale Gitter nicht mehr unendlich ausgedehnt und es verlängern sich die Punkte in die Richtung des Elektronenstrahles senkrecht zur Probenoberfläche. Man spricht bei diesen verlängerten Gitterpunkten von Stäbchen. Die Laue-Gleichung für konstruktive Interferenz lässt sich daher genauer formulieren zu

$$
\vec{K}=\vec{g}+\vec{s}
$$

Hierbei bezeichnet $\vec{s}$ den Schließungsvektor vom Punkt der exakten Laue-Bedingung zur Ewald-Kugel. Eine Erweiterung dieser Tatsache auf begrenzte Probendimensionen nicht nur senkrecht zur Elektroneneinstrahlrichtung wird im folgenden Kapitel erläutert.

\subsubsection{Beugung von dünnen Probenbereichen}

Die vorher beschriebene Tatsache, dass auch für Orientierungen wo $K=g$ nicht gilt Beugungsphänomene beobachtet werden können, lässt sich auf den Fall erweitern, wo das beugende Volumen in alle drei Raumrichtungen stark begrenzt ist. Der einfache oben beschrieben Fall wird der Dünne-Folie-Effekt genannt und ist Grundlage jedes Beugungsbildes. Darüber hinaus gibt es allerdings noch weitere Geometrien, die einen Einfluss auf das Beugungsbild haben. Dazu gehören

1. nicht-parallele Oberflächen

2. dünne Lamellen in der Matrix.

Bei keilförmigen Proben erzeugt jede Oberfläche jeweils ein auf dieser Oberfläche senkrecht stehendes Beugungsstäbchen. Nur für den exakten Fall $K=g$ ist dies im Beugungsbild also nicht zu erkennen. Für andere Werte von $s$ wird eine Aufspaltung des 
Beugungspunktes erkennbar. Diese Tatsache wird in Abbildung 2.9 veranschaulicht. Liegen in der Probe dünne Lamellen vor, gilt das Gleiche. Wieder kann für jede Grenz-

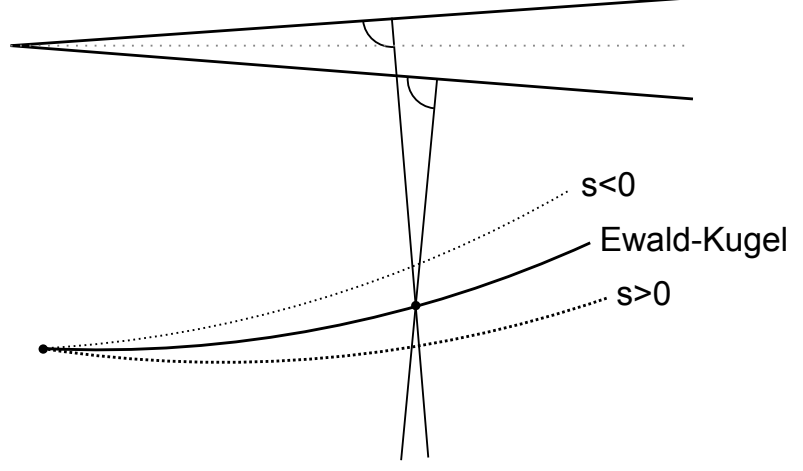

Abbildung 2.9: Beugung von einer keilförmigen Probe - Sind die Probenoberflächen nicht parallel, kann es zu einer Aufspaltung der Beugungspunkte führen, wenn $s \neq 0$ ist

fläche ein Beugungsstäbchen in den reziproken Raum gezeichnet werden. Der Effekt ist der Gleiche wie für nicht-parallele Oberflächen und man erhält zusätzliche Beugungspunkte. Für den Sonderfall, dass die dünne Lamelle parallel zum Elektronenstrahl liegt, ist das zugehörige Beugungsstäbchen senkrecht dazu. Für diese Geometrie schneidet die Ewald-Kugel das Stäbchen der Länge nach und wird als sogenannte "streaks"sichtbar. In fcc gibt es einige typische Defekt für die diese Besonderheiten im Beugungsbild beobachtet werden können. Dazu gehören Stapelfehler und dünne Zwillingslamellen. Stapelfehler können als sehr dünne Lamellen mit hcp Koordination aufgefasst werden. Von solchen planaren Defekten ist demnach zu erwarten, dass sie zu streaking führen sofern sie nahezu parallel zum Elektronenstrahl orientiert sind, für Zwillinge gilt dies analog. Zusätzlich befindet sich aber das Gitter innerhalb des Zwillings in einer speziellen Orientierung zur Matrix (vgl. Abschnitt 2.1). Wie in diesem Abschnitt gezeigt, ist das Material an einer $\{111\}$-Ebene gespiegelt. Im Beugungsbild verhält es sich nicht anders und man beobachtet alle Beugungsspots der Matrix und gespiegelt dazu die Punkte des Zwillings. Am anschaulichsten ist dies in der [110]-Zonenachse zu erkennen. Dort liegt der Normalenvektor der (1111)-Ebene senkrecht zum Elektronenstrahl. Spiegelt man nun die Punkte entlang dieser Achse, erhält man die Position der Beugungsreflexe für den verzwillingten Bereich. Einige Beispiele werden in Kapitel 5.1 gezeigt und erläutert. 


\subsection{Die Howie-Whelan Gleichungen}

Für die Herleitung der Howie-Whelan Gleichungen werden die Amplituden der transmittierten und der gebeugten Elektronenwellen beim Durchgang durch den Kristall betrachtet. Diese Wellen setzen sich aus den Einzelbeiträgen und Streuintensiäten jedes Atoms einer Einheitszelle zusammen. Dieser Amplitudenanteil beträgt

$$
A_{\text {Einheitszelle }}=\frac{e^{2 \pi i \vec{k} \cdot \vec{r}}}{r} \sum f_{i}(\theta) e^{2 \pi i \vec{K} \cdot \vec{r}_{i}} .
$$

Durch die Einführung des Strukturfaktors $F(\theta)$ statt über alle Atompositionen in der Einheitszelle zu summieren, lässt sich die Gleichung zu

$$
A_{\text {Einheitszelle }}=\frac{e^{2 \pi i \vec{k} \cdot \vec{r}}}{r} F(\theta)
$$

vereinfachen. Für die austretende Welle auf der unteren der Seite der Probe wird über alle Einheitszellen summiert. Die Summation ergibt die Amplitude der gebeugten Welle nach n Einheitszellen mit dem Abstand $a$

$$
\phi_{g}=\frac{\pi a i}{\xi_{g}} e^{-2 \pi \vec{K} \cdot \vec{r}_{n}} e^{2 \pi \vec{k}_{D} \cdot \vec{r}} .
$$

Dabei wurde mit $\xi_{g}$ eine Größe eingeführt, die durch den Strukturfaktor $\mathrm{F}_{g}$ materialabhängig ist und sowohl von dem Beugungswinkel $\theta$ als auch von der verwendeten Beschleunigungsspannung über $\lambda$ abhängt. Damit ist dieser Wert charakteristisch für jede Probe und jede Abbildebedingung. Man kann diese charakteristische Länge für jeden Vektor $\mathbf{g}$ berechnen mit der Formel

$$
\xi_{g}=\frac{\pi V_{c} \cos \theta_{B}}{\lambda F_{g}} .
$$

Nimmt man nun alle Formulierungen zusammen, lassen sich die Amplitudenänderungen für die transmittierte und die gebeugte Welle berechnen

$$
\begin{aligned}
& \frac{\phi_{0}}{d z}=\frac{\pi i}{\xi_{0}} \phi_{0}+\frac{\pi i}{\xi_{g}} \phi_{g} e^{2 \pi i s z} \\
& \frac{\phi_{g}}{d z}=\frac{\pi i}{\xi_{g}} \phi_{0} e^{-2 \pi i s z}+\frac{\pi i}{\xi_{0}} \phi_{g}
\end{aligned}
$$

Diese beiden Formeln stellen die Grundlage dar für die Betrachtung aller weiteren Beugungsphänomene in der Transmissionselektronenmikroskopie. Einige für diese Arbeit relevante Beispiele sollen im Verlaufe der nächsten Abschnitte detaillierter erläutert werden. 


\title{
2.4 Charakteristische Kontraste im TEM
}

\subsubsection{Biegekontrast}

Die wohl am häufigsten auftretenden Kontraste in Proben im TEM sind die, die von gebogenen Bereichen innerhalb eines Materials herrühren. Der Grund weshalb eine Verbiegung zu einem Kontrast führt ist in Abbildung 2.10 gezeigt. Trifft die Ausgangswelle
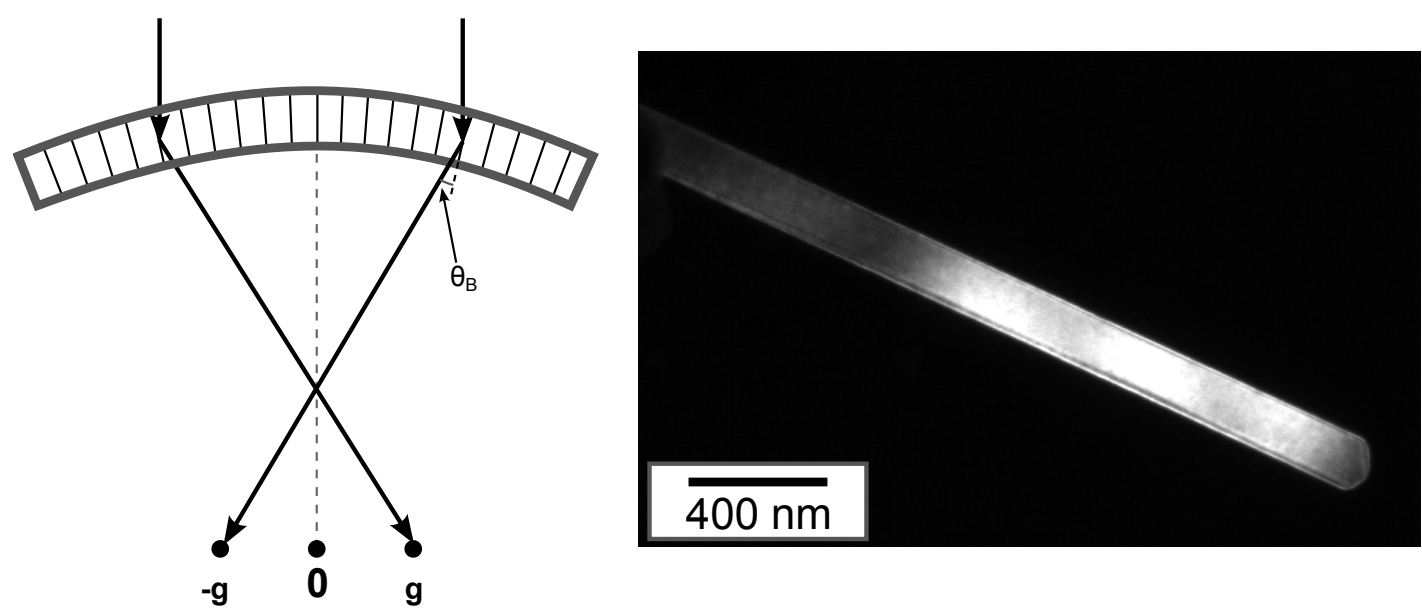

\begin{abstract}
Abbildung 2.10: schematische Darstellung zum Auftreten von Biegekontrasten Durch die lokale Erfüllung der Bragg-Bedingung wird in diesen Probenbereichen Intensität aus der einfallenden Welle in die gebeugte transferiert. Das nebenstehende Bild eines GoldDrahtes zeigt den typischen weichen Verlauf eines leicht verbogenen Drahtes im Dunkelfeld
\end{abstract}

auf den verbogenen Kristall wird an der Stelle, wo die Bragg-Bedingung erfüllt wird, ein Teil der Intensität aus dem Primärstrahl herausgebeugt. An anderen Stellen, wo der Winkel nicht exakt $\theta_{B}$ entspricht, wird entsprechend weniger oder sogar gar keine Intensität in Richtung $\vec{g}$ beobachtet. Schaut man sich nun die Intensitätsverteilung im Realraum an, indem man eine Blende so positioniert, dass nur Beiträge von $\vec{g}$ zur Bildformung beitragen, erkennt man einen Intensitätsverlauf innerhalb der Probe. Ein wesentliches Merkmal dieses Kontrastmechanismusses ist es, dass sich mit Verkippung der Probe auch der Punkt verändert, an dem der Elektronenstrahl im Winkel $\theta_{B}$ zur beugenden Ebene steht. Damit gehört der Biegekontrast zu den nicht ortsfesten Kon- 


\section{THEORETISCHE GRUNDLAGEN}

trasten im TEM. Dieses Merkmal ist von entscheidender Bedeutung bei der Beurteilung von Dunkel- und Hellfeldbildern von Proben.

\subsubsection{Kontrast von planaren Defekten}

Dieser Abschnitt soll sich mit der Frage beschäftigen, warum Translationen $\vec{R}$ im realen Gitter überhaupt Kontraste hervorrufen. Um diese Frage zu beantworten, betrachten wir den allgemeinen Fall einer Translation in einer Probe. Die Positionen der Gitterpunkte $r^{\prime}$ in dem verschobenen Bereich lassen sich durch die Vorschrift

$$
\overrightarrow{r_{n}^{\prime}}=\overrightarrow{r_{n}}+\vec{R}
$$

beschreiben. Setzt man diese einfache Korrektur in die Gleichung 2.22 ein, erhält man nach einer kurzen Umformung für den Exponenten:

$$
\begin{aligned}
\vec{K} \cdot \overrightarrow{r_{n}^{\prime}} & =(\vec{g}+\vec{s}) \cdot\left(\overrightarrow{r_{n}}+\vec{R}\right) \\
& =\vec{g} \cdot \overrightarrow{r_{n}}+\vec{g} \cdot \vec{R}+\vec{s} \cdot \overrightarrow{r_{n}}+\vec{s} \cdot \vec{R}
\end{aligned}
$$

In dieser Summe sind einige Teile bekannt. Der erste und dritte Summand tritt auch ohne Translation auf, der letzte Summand lässt sich vernachlässigen, da dessen Beitrag verhältnismäßig klein ist, wenn man unter Zweistrahlbedingung arbeitet. Der zweite Term stellt somit die Neuerung dar. Vergleicht man die so modifizierten Gleichungen mit den Howie-Whelan Gleichungen 2.24 haben sie im Wesentlichen dieselbe Form:

$$
\begin{aligned}
\frac{\phi_{0}}{d z} & =\frac{\pi i}{\xi_{0}} \phi_{0}+\frac{\pi i}{\xi_{g}} \phi_{g} e^{2 \pi i(s z+\vec{g} \cdot \vec{R})} \\
\frac{\phi_{g}}{d z} & =\frac{\pi i}{\xi_{g}} \phi_{0} e^{-2 \pi i(s z+\vec{g} \cdot \vec{R})}+\frac{\pi i}{\xi_{0}} \phi_{g}
\end{aligned}
$$

Der einzige Unterschied ist ein zusätzlicher Phasenterm mit der Phase

$$
\alpha=2 \pi \vec{g} \cdot \vec{R} .
$$

Diese Beschreibung ist für das Verständnis von Stapelfehlern im TEM von entscheidender Bedeutung.

Stapelfehler in einem kubisch flächenzentrierten Kristall liegen wie in Abschnitt 2.1.1 beschrieben auf (111)-Ebenen. Damit ist auch der Translationsvektor vorbestimmt und 
kann nicht jeden beliebigen Wert annehmen. Für die fcc Struktur ist dieser Wert abhängig von der genauen Natur des Stapelfehlers. Ein intrinsischer Stapelfehler unterscheidet sich von einem extrinsischen Stapelfehler in der Orientierung der Translation relativ zum Rest des Kristalls. Damit gilt:

$$
\vec{R}= \pm 1 / 3[111]
$$

Aus dieser Beschreibung wird auch deutlich, dass der zusätzliche Phasenterm für bestimmte Abbildebedingungen wegfällt und kein Kontrast von dem Defekt zu erwarten ist. Dies ist zum Beispiel bei $\vec{g}=(02 \overline{2})$ der Fall, da das Skalarprodukt gleich Null ist. Für andere Abbildebedingungen ist der Defekt hingegen sichtbar, dabei ist das Aussehen sehr charakteristisch.

Befindet sich der Defekt in der Tiefe $t_{1}$ im Kristall kann die Gleichung 2.29 benutzt werden um die Phasen der einzelnen Wellen zu berechnen. Dazu integriert man zum Beispiel die Beiträge in der gebeugten Welle jeweils bis zur Dicke der Probe t auf:

$$
\begin{aligned}
\phi_{g} & =\frac{\pi i}{\xi_{g}}\left\{\int_{0}^{t_{1}} e^{-2 \pi s z} d z+\int_{t_{1}}^{t} e^{\alpha} e^{-2 \pi s z} d z\right\} \\
& =\frac{i}{\xi_{g} s} e^{-\pi i s t_{1}}\left\{\sin \pi t_{1} s+e^{-i \alpha} e^{-\pi i s t} \sin \pi\left(t-t_{1}\right) s\right\} \\
& =\frac{i}{\xi_{g} s} e^{-i \alpha / 2} e^{-\pi i s t}\left\{\sin \left(\pi t s+\frac{\alpha}{2}\right)-\sin \frac{\alpha}{2} e^{2 \pi i s t\left(t / 2-t_{1}\right)}\right\}
\end{aligned}
$$

Die Intensitätsverteilung dieses Phasenverlaufes erhält man dann indem man die Gleichung quadriert und mit Hilfe von $\mathrm{z}=1 / 2 \mathrm{t}$ - $\mathrm{t}_{1}$ vereinfacht.

$$
I=\frac{1}{\left(\xi_{g} s\right)^{2}}\left[\sin ^{2}\left(\pi t s+\frac{\alpha}{2}\right)+\sin ^{2} \frac{\alpha}{2}-2 \sin \frac{\alpha}{2} \sin \left(\pi t s+\frac{\alpha}{2}\right) \cos 2 \pi s z\right]
$$

Aus dieser Formulierung erkennt man nun auch das charakteristische Aussehen eines Stapelfehlers. Der einzige Term, der in der obigen Gleichung von der Tiefe abhängt, ist der $\cos (2 \pi \mathrm{sz})$. Er gibt vor, wie sich die Intensität mit zunehmender Tiefe verhält. Die Intensität moduliert also mit der Periodizität von $\mathrm{s}^{-1}$ und ist symmetrisch um die Probenmitte bei $\mathrm{z}=0$. Ein Besipiel eines Stapelfehler in einem Gold-Nanodraht ist in Abbildung 2.11 gezeigt. 


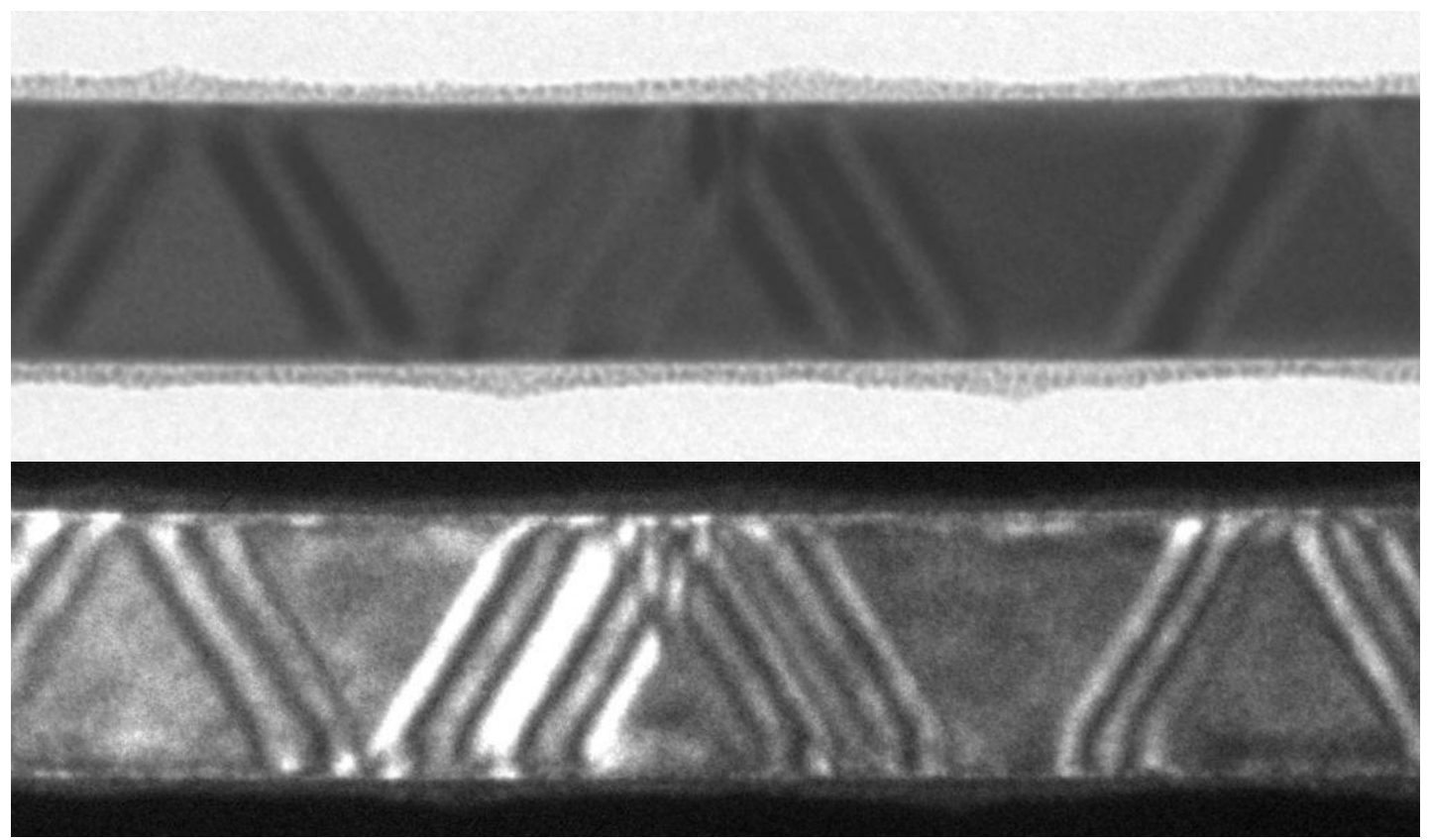

Abbildung 2.11: Stapelfehler in einem Gold Nanodraht - Das Beispiel zeigt den typischen oszillierenden Kontrastverlauf im Hellfeld (oben) und Dunkelfeld (unten) eines Stapelfehlers. Deutlich erkennbar ist die Symmetrie der Oszillation um die Mitte des Defektes. 


\subsubsection{Versetzungskontrast im TEM}

Eine weiterer wichtiger Kristallbaufehler ist die Versetzung. Im Abschnitt 2.1 wurde auf die Bedeutung der Versetzung bei der Verformung von Kristallen eingegangen. In diesem Kapitel soll nun erläutert werden, warum Versetzungen im TEM einen Kontrast hervorrufen und wann dieser sichtbar ist.

Die Gleichungen 2.29 zeigen, dass eine Störung in der Ordnung des Gitters einen zusätzlichen Phasenterm verursacht. Dabei war allerdings die Störung im Falle eines Stapelfehlers eine einfache Translation. Dennoch kann dieses Konzept auch für kompliziertere Störungen verwendet werden. Im Falle einer Versetzung mit Burgersvektor $\vec{b}$ in einem isotropen Material kann diese Translation zum Beispiel beschrieben werden durch

$$
\vec{R}=\frac{1}{2 \pi}\left(\vec{b} \phi+\frac{1}{4(1-\nu)}\left\{\overrightarrow{b_{e}}+\vec{b} \times \vec{u}(2(1-2 \nu) \ln r+\cos 2 \phi)\right\}\right) .
$$

Diese allgemeine Form lässt sich für die zwei bekannten Spezialfälle vereinfachen. Für den Fall, dass eine reine Schraubenversetzung vorliegt. vereinfacht sich der Term zu

$$
\vec{R}(x, z)=\frac{\vec{b}}{2 \pi} \tan ^{-1}\left(\frac{z-z_{d}}{x}\right) .
$$

Hierbei bezeichnet $\mathrm{z}_{d}$ die Lage des Versetzungskerns innerhalb der Probe.

Für die Beschreibung einer Stufenversetzung sind zwei Komponenten für die Translation nötig. Hier erhält man

$$
\begin{aligned}
& \overrightarrow{R_{x}}=\frac{\vec{b}}{2 \pi}\left[\tan ^{-1} \frac{y}{x}+\frac{x y}{2(1-\nu)\left(x^{2}+y^{2}\right)}\right] \\
& \overrightarrow{R_{y}}=-\frac{\vec{b}}{2 \pi}\left[\frac{1-2 \nu}{4(1-\nu)} \ln \left(x^{2}+y^{2}\right)+\frac{x^{2}-y^{2}}{4(1-\nu)\left(x^{2}+y^{2}\right)}\right]
\end{aligned}
$$

Deutlich erkennbar ist hier, dass die Störung logarithmisch in $\mathrm{r}=\sqrt{x^{2}+y^{2}}$ ist. Dies bedeutet, dass nahe am Versetzungskern die Verzerrung am größten ist.

Setz man nun beiden Formulierungen in den Phasenterm 2.31 ein, wird deutlich, dass der erzeugte Kontrast für eine Versetzung im Wesentliche vom Skalarprodukt $\vec{g} \cdot \vec{b}$ abhängt. Diese Tatsache lässt sich einfach verstehen, wenn man sich zum Beispiel die Geometrie einer reinen Stufenversetzung anschaut. In Abbildung 2.12 ist eine solche Versetzung schematisch gezeigt, bei der man entlang des Linienelementes schaut. Damit liegt der Burgersvektor in der Zeichenebene. Den größten Anteil am Kontrast haben diejenigen Ebenen nahe am Versetzungskern, die gerade in Bragg-Bedingung mit der 
einfallenden Elektronenwelle stehen. Das Skalarprodukt aus $\vec{g} \cdot \vec{b}$ ist somit $\neq 0$ und der Kristallbaufehler wird sichtbar, da er zu einer Phasenverschiebung führt.
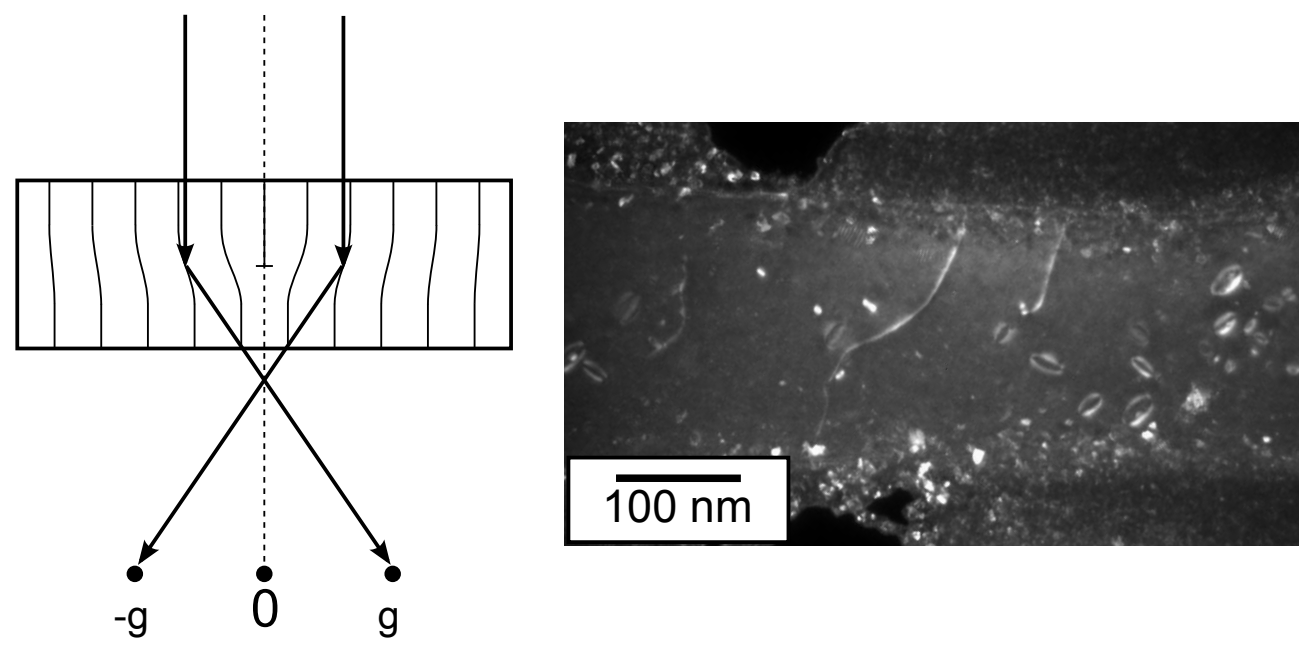

Abbildung 2.12: Schematische Zeichung und Beispiele zum Kontrastmechanismus einer Versetzung - Um den Versetzungskern wird an gebogenen Ebenen die BraggBedingung erfüllt und die Elektronenwelle gebeugt. Im Dunkelfeldbild (rechts) kann dann unter bestimmten Abbildebedingungen die Veretzung sichtbar gemacht werden. Hier gezeigt sind als helle Linien Versetzungen in einer verformten Aluminium Probe. 


\section{3}

\section{Die Nanodrähte}

Wie schon in der Motivation zu dieser Arbeit erwähnt wurde, sind sehr hohe Anforderungen an die Proben zu stellen. Dies liegt daran, dass störende Einflüsse, wie etwaige vorhandene Defekte, ausgeschlossen werden sollten. Somit war ein wesentlicher Teil dieser Arbeit die Auswahl von geeignetem Probenmaterial. Es wurde darauf Wert gelegt, dass die verwendeten Proben vor den eigentlichen Verformungsexperimenten hinreichend gut charakterisiert werden. Diese Charakterisierung fand zum einen im REM statt, wo mit einfachen Mitteln die Längen und Durchmesser der Drähte bestimmt werden konnten. Zum anderen wurden durch TEM Untersuchungen die Kristallographie und Mikrostruktur einer Vielzahl von Drähten untersucht. In den folgenden Abschnitten wird das so gewonnene Bild der Proben dargestellt.

Die Nanodrähte wurden aus einer Kooperation mit der Gruppe von Gunther Richter am zentralen Dünnschichtlabor am Max-Planck-Institut für Intelligente Systeme (früher: für Metallforschung) in Stuttgart bezogen. Dort können eindimensionale Strukturen aus unterschiedlichen Metallen produziert werden. An Kupfer konnte bereits detailiert gezeigt werden, dass diese Proben eine außerordentlich hohe kristalline Perfektion besitzen [34]. Für die Untersuchungen in dieser Arbeit war neben der Mikrostruktur wichtig, dass durch eine ausreichende Länge der Drähte ein problemloser Transfer gewährleistet war. Dieser war außerdem dann besonders einfach zu realisieren, wenn die Drähte als freistehende Objekte auf einem Substrat vorlagen. Alle diese Voraussetzungen waren nötig, damit der Draht seine Perfektion während des Transfers nicht verlor. Damit waren dann erst aussagekräftige Experimente durchführbar.

Da der Transferprozess zentraler Bestandteil des Experimentes ist, wird dieser im Detail 


\section{DIE NANODRÄHTE}

in Abschnitt 4.1 beschrieben.

\subsection{Vorcharakterisierung im REM}

Für die Vorcharakterisierung der Drähte und um einen ersten Eindruck von der Probenqualtität zu erhalten, wurden am NovaNanolab 600 von der Firma FEI verschiedene Aufnahmen der Drähte angefertigt. Mit diesen Aufnahmen war man in der Lage, die Orientierung der Drähte auf dem Substrat sowie deren räumliche Anordung und Länge einzuschätzen. Für die in dieser Arbeit verwendeten Drähte wurde stets das gleiche Material verwendet, alle Drähte wurden somit in einem Depositionsprozess hergestellt. In diesem Fall wurde als Substrat ein Wolframdraht mit einem Durchmesser von 0,3 mm verwendet. Nach der Herstellung waren im REM die einzelnen Drähte klar erkennbar. Neben quasi 1-dimensionalen Strukturen lagen auch plattenartige Strukturen wie auch Nanopartikel auf der gesamten Substratoberfläche vor. Ein typisches Bild dieser Probe ist in Abbildung 3.1 gezeigt. Da die Drähte einige Vorgaben hinsichtlich Länge

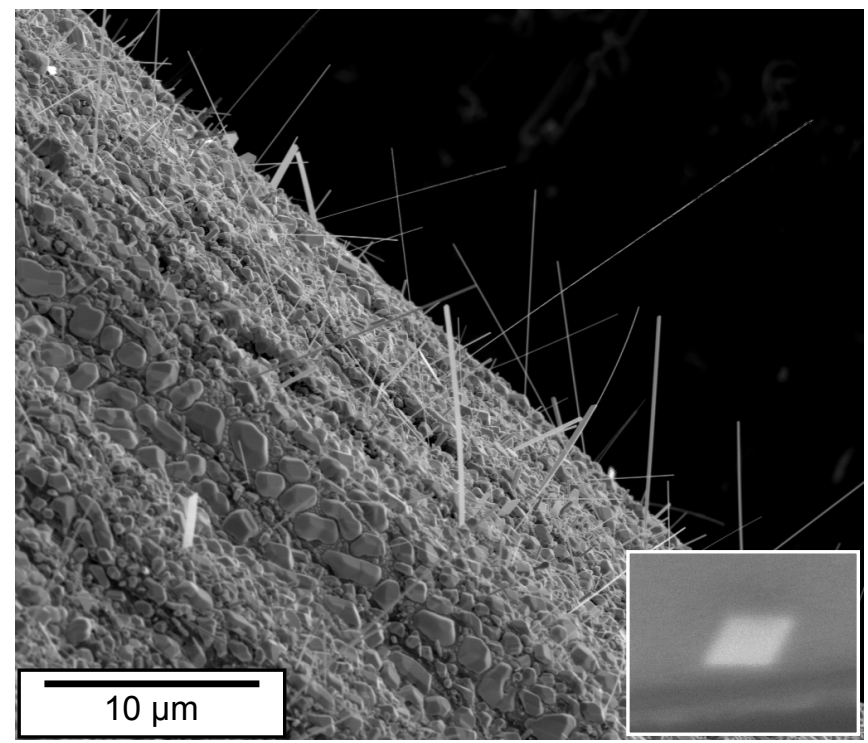

Abbildung 3.1: REM-Aufnahme der Gold-Nanodrähte - Eine Vielzahl von freistehenden Nanodrähten mit komplizierteren Formen befinden sich auf der Substratoberfläche nach dem Herstellungsprozess. Das eingesetze Bild zeigt einen typischen Querschnitt der Drähte.

und Durchmesser für einen erfolgreichen Transfer (vgl. Abschnitt 4.1) erfüllen muss- 
ten, waren nicht alle Drähte für die Benutzung in einem Zugversuch geeignet. War der Winkel unter dem der Draht auf dem Substrat stand nahe an $90^{\circ}$ und die Länge groß genug, konnte er transferiert werden. Durch diese Vorgaben waren die vorhandenen Drahtdurchmesser bestimmt. Als kleinstmögliche Probe erwiesen sich Drähte mit einer Breite von 40 nm. Darunter lagen zwar drahtähnliche Strukturen auf der Probe vor, deren Länge war aber nicht hinreichend groß für einen Transfer. Die größtmöglichen Drähte hatten Breiten bis $300 \mathrm{~nm}$. Es zeigte sich, dass mit zunehmender Dicke die Strukturen dazu tendieren, eher plattenhaften Charakter auszubilden anstelle weiteren Dickenwachstums.

Durch Querschnittspräparation war es möglich, auch Informationen über Kristallstruktur senkrecht zur Wachstumsachse zu gewinnen. Hier zeigte sich, dass in den meisten Fällen rhomboide Querschnittsflächen vorlagen [24]. Ein Beispiel einer solche Querschnittsfläche ist in der eingebetteten Darstellung in Abbildung $3.1 \mathrm{zu}$ sehen. Die Wulff-Konstruktuion für einen solchen Kristall sieht vor, dass die Aussenfacetten im Wesentlichen durch $\{111\}$-Facetten gebildet werden. Eine genaue Konstruktion ist in [24] für Gold und in [34] für Kupfer durchgeführt. Zusätzlich werden die spitzen Winkel zwischen zwei $\{111\}$-Ebenen durch $\{100\}$-Facetten abgeschnitten. Dadurch ergibt sich ein gestutztes Rhomboid. Dieses Abschneiden der spitzen Facetten lag jedoch nicht bei allen Drähten vor. Auch konnte die Länge der $\{100\}$-Facette, sofern sie vorhanden war, von Draht zu Draht variieren.

\subsection{Vorcharakterisierung im TEM}

Die Vorcharakterisierung sowie alle späteren Zugversuche wurden am TEM CM 30 der Firma Philips bei 300kV Beschleunigungsspannung durchgeführt. Für eine schnelle Charakterisierung der Drähte und deren Wachstumsrichtung war eine hohe Statistik nötig. Da der Einzeltransfer (vgl. 4.1) deutlich zu zeitaufwendig gewesen wäre, wurden diese Übersichtsproben anders präpariert. Dazu kam ein herkömmliches TEM-Netzchen zum Einsatz, dass mit einem löchrigen Kohlenstofffilm benetzt war. Dieses Netzchen wurden dann mittels einer Pinzette über das Substrat gestrichen. Im TEM zeigte sich dann, dass allein durch diesen Prozess Probenmaterial vom Substrat gelöst werden konnte und auf dem Kohlenstofffilm haften blieb. Durch diesen verhältnismäßig unkontrollierten Transfer von Probenmaterial konnten keine Informationen über die anfäng- 


\section{DIE NANODRÄHTE}

liche Defektstruktur erhalten werden. Dennoch kann dieses Verfahren einen Eindruck von der Kristallinität und insbesondere der Wachstumsrichtung geben. In Abbildung 3.2 sind vier Drähte dargestellt, bei denen mit Hilfe des Beugungsbildes ermittelt wurde, wie die Wachstumsrichtung ist. In allen Fällen lag diese entlang $<110>$. Zusätzlich zu dieser Vorcharakterisierung wurde jeweils vor Beginn eines Experimentes die Orientierung separat bestimmt (vgl. dazu Kapitel 4.2.2).

Die Beugungsbilder zeigten, dass alle Drähte einkristallin waren. Die einzigen Defekte waren Zwillinge, die parallel zur Drahtachse lagen, wie es in Abbildung 3.2 im Falle des Drahtes rechts unten gezeigt ist.

Für die Zugversuche war die Länge der Drähte unerheblich, sofern sie über $7 \mu \mathrm{m}$ lag. Dies war die minimale Länge, die nötig war, um einen erfolgreichen Transfer auf die $\mathrm{CuBe}$ Rahmenstruktur zu gewährleisten. Eine wichtige Größe stellte allerdings auch der Durchmesser der Drähte dar. Da dies aufgrund der rhomboedrischen Querschnittsform keine einfache Zahl ist, muss für die Beschreibung der Breite ein anderer Parameter gewählt werden. Es zeigte sich dabei im Laufe der Versuche, dass bedingt durch den Halter meist nur eine niedrig indizierte Zonenachse für die Zugversuche zugänglich war. In dieser Zonenachse wurde dann die erscheinende Breite als charakterisierende Größeangabe verwendet. Bedingt durch die statistische Verteilung der Orientierung nach dem Transfer war dies auch nicht zwangsläufig die gleiche Zonenachse, sodass hier mit Abweichungen zu rechnen war. Wird im Folgenden von der Breite eines Drahtes gesprochen, ist damit nicht eine spezielle Facettenlänge gemeint, sondern vielmehr die projizierte Breite in einer speziellen Zonenachse. Abbildung 3.3 veranschaulicht diese Tatsache. 


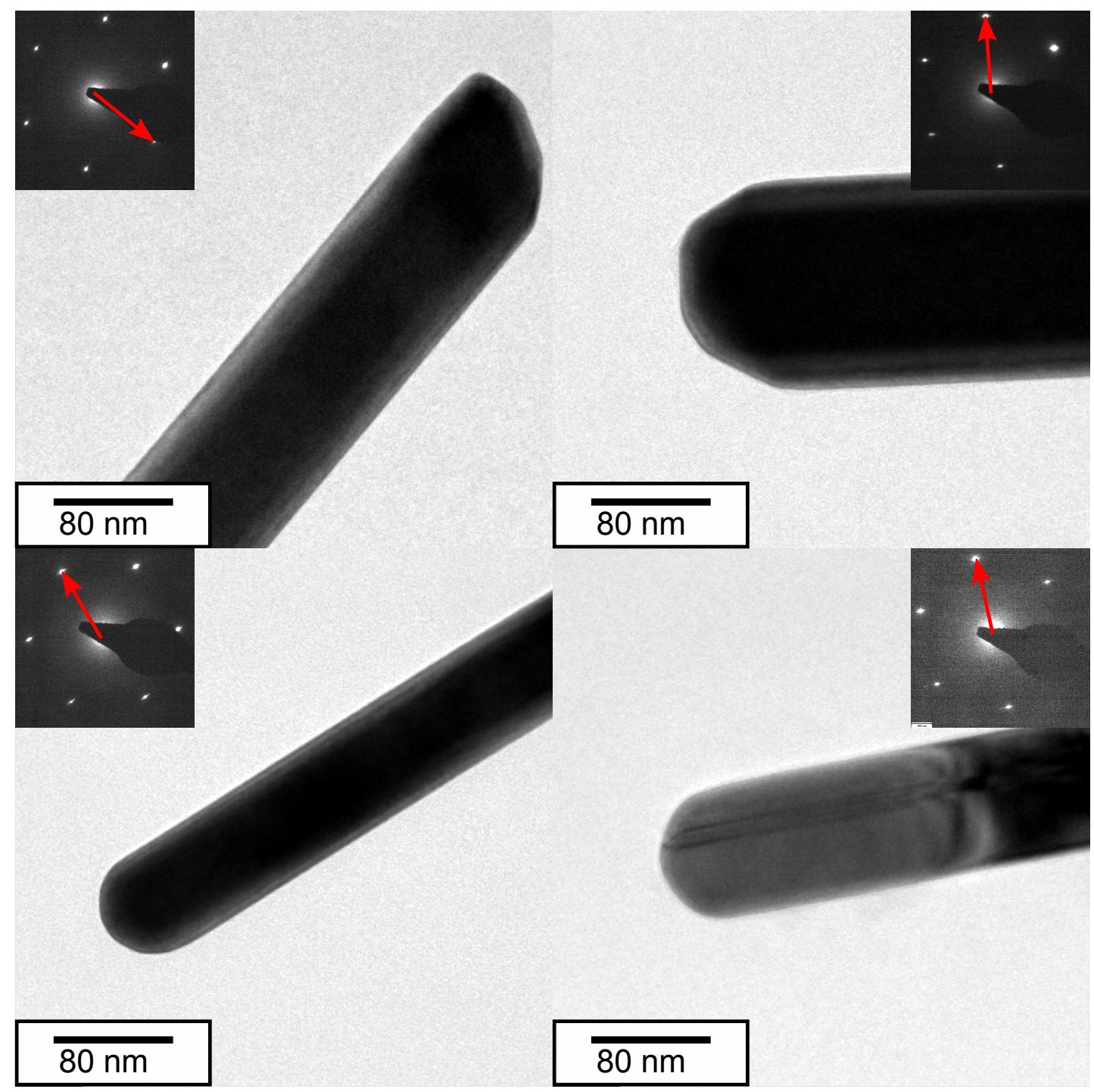

Abbildung 3.2: TEM Aufnahmen zur Orientierungsbestimmung der GoldDrähte - Verschiedene Hellfeldaufnahmen mit zugehörigen Beugungsbildern, mithilfe derer die Wachstumsrichtung der Drähte bestimmt wurde. Alle Drähte zeigten ein Wachstum entlang $<110>$. Im Bild rechts unten ist ein Defekt parallel zur Drahtachse erkennbar. 

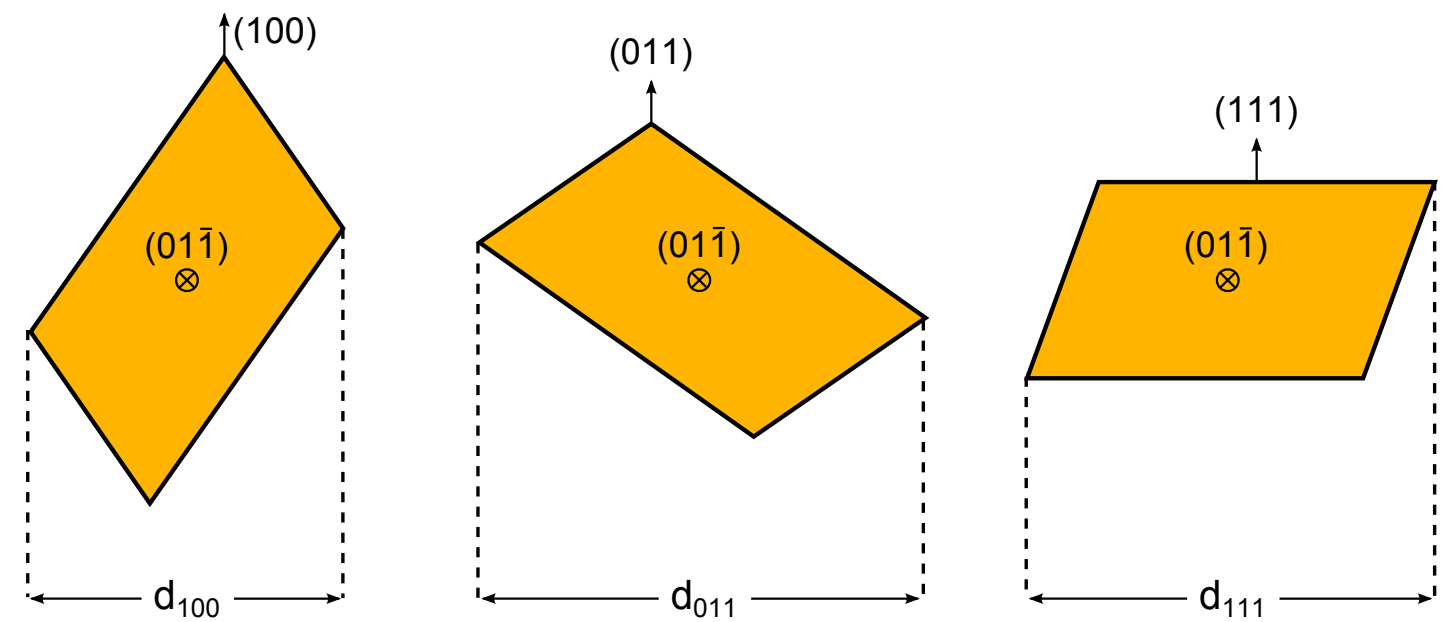

Abbildung 3.3: Breitenbestimmung der Drähte - Durch die rhomboedrische Querschnittsform ist die Breite der Drähte nicht klar definiert. Daher wurde für diese Arbeit die erscheinende Breite des Drahtes in einer durch Verkippung im TEM erreichbaren niedrig indizierten Zonenachse als beschreibende Größe verwendet.

\subsection{1 Überstruktur in der $\{111\}$-Zonenachse}

Bei der Charakterisierung der Drähte im TEM ist eine Besonderheit beobachtet worden, die von der erwarteten einkristallinen Struktur abweicht. In manchen Fällen konnten in der (111)-Zonenachse Beugungsreflexe an verbotenen Positionen beobachtet werden. Die Auswahlregel für ein fcc-Gitter lässt nur dann einen Reflex zu, wenn alle Indizes gerade oder alle ungerade sind. Somit sind in der ersten Ordnung der (111)-Zonenachse nur Reflexe vom Typ $<220>$ zu erwarten. In einigen Fällen konnten jedoch auch schwächere Reflexe an der rechnerischen Position $1 / 3<422>$ beobachtet werden. In Abbildung 3.4 ist ein normales Beugungsbild gezeigt und im Vergleich dazu eines mit der beschriebenen Überstruktur. Für den Fall von Gold sind solche Überstrukturreflexe schon lange in der Literatur beschrieben worden [35]. Lediglich die Interpretation der Beobachtungen ist nicht eindeutig geklärt. Die gängigsten Erklärungen für die zusätzlichen Beugungspunkte sind

- Doppelbeugung an Zwillingen in verbogenen Proben [36]

- Bruch in der fcc Stapelfolge durch monoatomare Oberflächenstufen [37]

- Oberflächenrekonstruktion [38] 


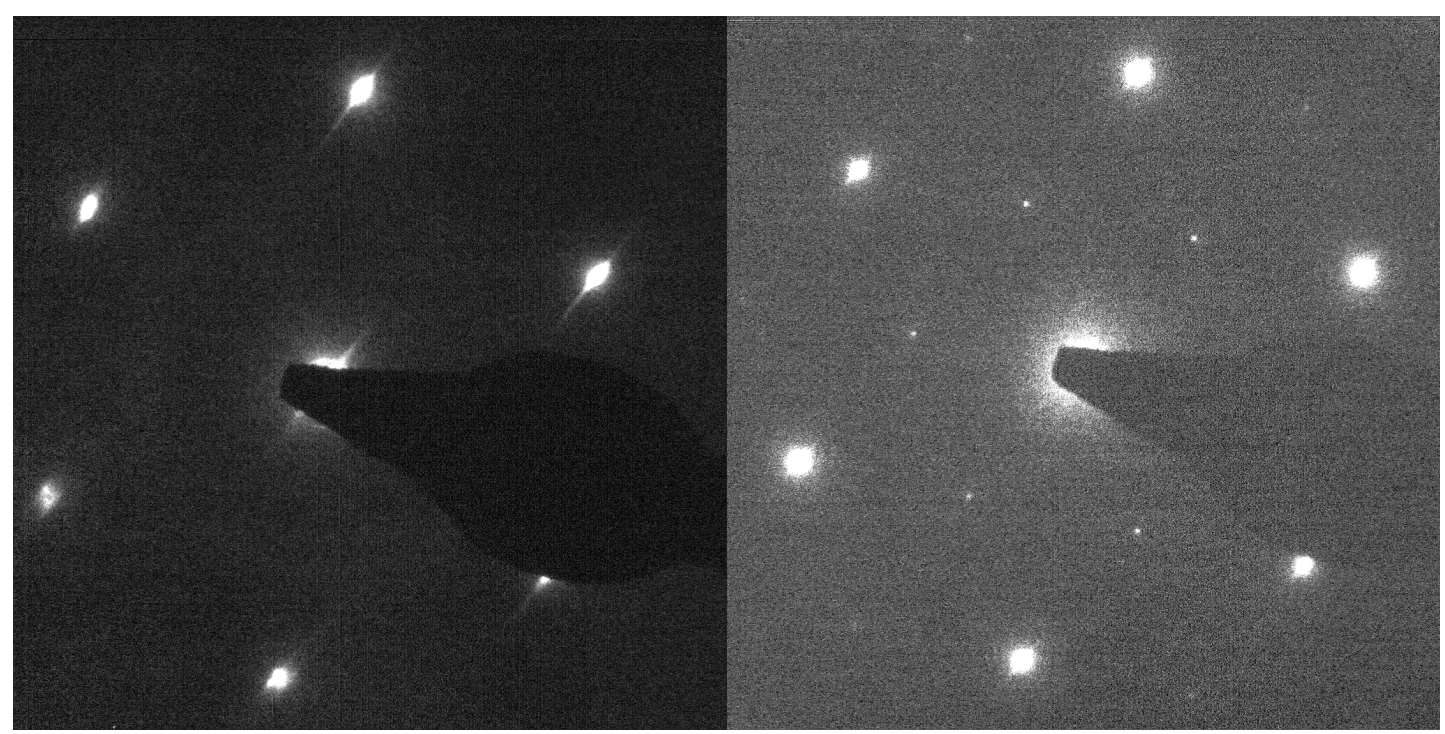

Abbildung 3.4: Beugungsbilder der (111)-Zonenachse - Links ist das ungestörte Beugungsbild der (111)-Zonenachse eines fcc-Gitters zu sehen. Das rechte Bild zeigt die im Text erwähnte Überstruktur.

- Senkrecht zur Elektroneneinstrahlrichtung liegende Stapelfehler und Zwillinge [39]

Sicherlich sind aus dieser Liste einige Punkte nicht von Relevanz für die verwendeten Proben. Zum Beispiel lag in keiner Probe eine hinreichend große Verbiegung vor, um die Bragg-Bedingung in zwei benachbarten Zwillingen gleichzeitig zu erfüllen. Daher verbleiben als relevante Erklärungen die Punkte 2-4. Primäres Ziel dieser Arbeit ist es jedoch nicht, sich an der Lösung der Klärung des Ursprungs der Beugungspunkte zu beteiligen, sondern vielmehr abzuschätzen, ob das Vorhandensein der Überstruktur zu einem veränderten Verformungsverhalten führt. Daher wird auf diesen Aspekt an der jeweiligen Stelle in der Darstellung der Ergebnisse eingegangen. Eine zusammenfassende Darstellung der Auswirkungen wird am Ende von Kapitel 5 geliefert. 
3. DIE NANODRÄHTE 


\section{4 \\ Experimentelle Grundlagen}

Dieses Kapitel soll einen Überblick über die Verfahrensweise geben, wie Proben für unterschiedliche Verformungsgeometrien realisiert wurden. Schwerpunkte sind die nötigen Schritte für die Vorpräparation und die experimentelle Umsetzung der Zugversuche an sich. Darüber hinaus wird erläutert, wie Biegeversuche im TEM realisiert wurden. Es wird außerdem aufgezeigt, welche technischen Limitierungen der gewählte Aufbau hat und wie sich diese auf die Ergebnisse der Zugversuche auswirken.

\subsection{Vorpräparation von Verformungsproben}

Für die Durchführung der verschiedenen Experimente im TEM wurden unterschiedliche Geometrien gewählt, die jeweils die Realisierung des gewünschten Spannungszustandes zuließen. Dies bedeutet natürlich auch, dass nicht nur eine Art der Probenpräparation in dieser Arbeit durchgeführt wurde, sondern vielmehr die einzelnen Schritte an das gewünschte Experiment angepasst wurden. Dennoch verläuft der Präparationsschritt im ersten Teil für alle Geometrien gleich. Auf die spezifischen Unterschiede wird dann in den jeweiligen Unterpunkten eingegangen.

Die Manipulation der hier verwendeten Gold-Nanodrähte wurde in einem Rasterelektronenmikroskop von FEI (Nova NanoLab 600) durchgeführt. Dabei kam der Mikromanipulator Omniprobe zum Einsatz. Dieser hat eine Spitze aus Wolfram, deren Radius bis auf unter $100 \mathrm{~nm}$ gedünnt werden kann.

Diese Spitze wird nun mit Hilfe der Tischbewegung von unten mit dem gewünschten Draht an seiner Wurzel in Berührung gebracht. Ist der Kontakt hergestellt, erfolgt ei- 


\section{EXPERIMENTELLE GRUNDLAGEN}

ne Fixierung des Drahtes mit einem etwa $200 \mathrm{~nm}$ x $800 \mathrm{~nm}$ großen Platin Pflaster. Dieses wird mit Hilfe des Elektronenstrahls und unter Einlassen eines Gas-precursors auf der Probe abgeschieden. Eine Bewegung der Omniprobe senkrecht vom Substrat weg bewirkt dann, dass der Draht abreißt, ohne dass das freistehende Ende mechanisch belastet wird. Dieser Vorgang ist in Abbildung 4.1 dargestellt. Damit ein problemloses Befestigen des Drahtes im zweiten Schritt auf der gewünschten Unterlage stattfinden kann, muss dafür gesorgt werden, dass die Drahtachse horizontal verläuft. Um dies zu erreichen wird die Omniprobe entlang ihrer Achse gedreht. Mit Hilfe von Längenmessungen am Draht und dem Vergleich des Höhenwertes an Spitze und Omniprobe, der über den Fokus ermittelt werden kann, wird der Draht horizontal ausgerichtet.

Nach diesen vorbereitenden Schritten ist der Draht in der richtigen Position, um auf
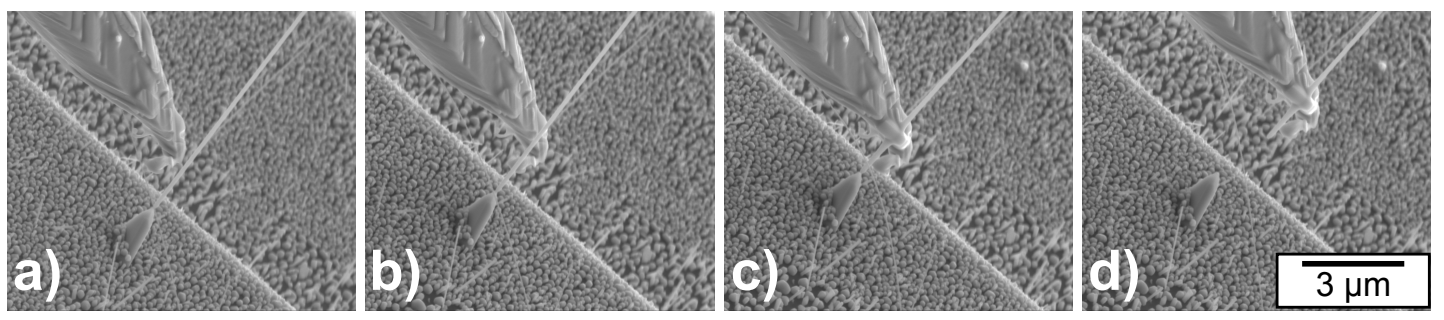

Abbildung 4.1: Vorbereitende Schritte zum Drahttransfer - REM Bilder von den vorbereitenden Schritten für einen Drahttransfer: a) Annäherung des Manipulators b) Positionierung unterhalb des Drahtes c) Depostiion eines Platinpflaster am Berührungspunkt zur Befestigung d) Ablösen vom Substrat

den gewünschten Unterlagen abgelegt zu werden. Diese speziellen Schritte werden in den nächsten beiden Unterkapiteln dargestellt.

\subsection{Zugversuche auf CuBe-Rahmen}

Die verwendeten Kupfer-Beryllium-Rahmen werden als vorstrukturierte Metallfolie geliefert [40], aus der die individuellen Rahmen mit Hilfe einer Pinzette herausgebrochen werden können. Direkt nach dem Herauslösen sind sie allerdings noch nicht für den Einsatz als Zugunterlage geeignet, da in dem Herstellungsprozess keine Dünnung bis in den elektronentransparenten Bereich vorgenommen werden kann. Daher muss vor dem eigentlichen Einsatz ein Fenster für die elektronenmikroskopischen Untersuchungen geschaffen werden. Dieses Fenster wurde im FIB von der herstellerseitig vorgedünnten 
Rückseite des Rahmens aus geschnitten. Die Abmessungen dieses Fenster waren typischerweise 5 bis $6 \mu \mathrm{m}$ in der Länge und etwa $30 \mu \mathrm{m}$ in der Breite. Die obere Grenze der Abmaße ist dabei im Wesentlichen gegeben durch die Präparationsdauer und die Stabilität der Rahmenstruktur im Zugversuch. Wählt man das Fenster zu breit, ist der Rahmen zu stark geschwächt und damit die Dehnung zu groß, um einen kontrollierten Zugversuch durchführen zu können. Um allerdings nicht für jeden Zugversuch einen neuen CuBe-Rahmen vorpräparieren zu müssen, wurde eine Breite von etwa $30 \mu \mathrm{m}$ gewählt. So war es möglich, mehrere Drähte nacheinander auf derselben Struktur zu testen.

Wie man auf den REM Bildern aus dem ersten Teil des Transfers erkennen kann, ist der Draht in der Regel nicht direkt an der äußersten Spitze des Manipulators befestigt, sondern sitzt vielmehr ein Stück weit auf dem Manipulator. Dies hat zur Folge, dass keine Ablage auf eine plane Oberfläche möglich ist. Um diesem Problem zu begegnen, ist zusätzlich zu dem durchgehenden Fenster auch noch eine Vertiefung direkt vor diesem geschnitten worden. Während des Transferprozesses kann dort die Spitze des Mikromanipulators ein Stück weit unter die Oberfläche eindringen und den Draht in Kontakt mit dem Rahmen bringen. Die fertig präparierte CuBe-Struktur sieht demnach aus, wie in Abbildung 4.2 gezeigt.

Im nächsten Schritt wird der aus Abschnitt 4.1 vorbereitete Draht mit der CuBeStruktur ausgerichtet. Dazu wird der Tisch unterhalb des Drahtes so gedreht, dass die Drahtachse und die Längsachse der Rahmenstruktur parallel zueinander liegen. Die Ausrichtung wird dabei mit Hilfe einer Winkelmessung, die das REM als Werkzeug mitliefert, kontrolliert. Auf diesem Wege kann eine Genauigkeit von unter $1^{\circ}$ erreicht werden. In kleinen Schritten wird danach der Tisch mit dem CuBe-Rahmen dem Draht entgegen nach oben bewegt bis beide Kontakt haben. Zwei Platin Pflaster mit Dimensionierungen wie im vorigen Abschnitt fixieren den Draht dann auf der Unterlage. Wird nun der Mikromanipulator wieder entfernt, reißt der Draht in dem kurzen Stück zwischen Fixpunkt auf dem Rahmen und Fixpunkt an dem Manipulator. Diese Prozesschritte sind in einer Serie von REM Bildern in Abbildung 4.3 dargestellt. Der Vorteil dieses Transferprozesses ist, dass der Teil vom Draht, der für die Verformung von Interesse ist und über dem Loch liegt nur sehr geringe mechanische Spannungen erfahren hat. Durch TEM Analysen jeweils vor den Zugversuchen kann darüber hinaus bestätigt 


\section{EXPERIMENTELLE GRUNDLAGEN}

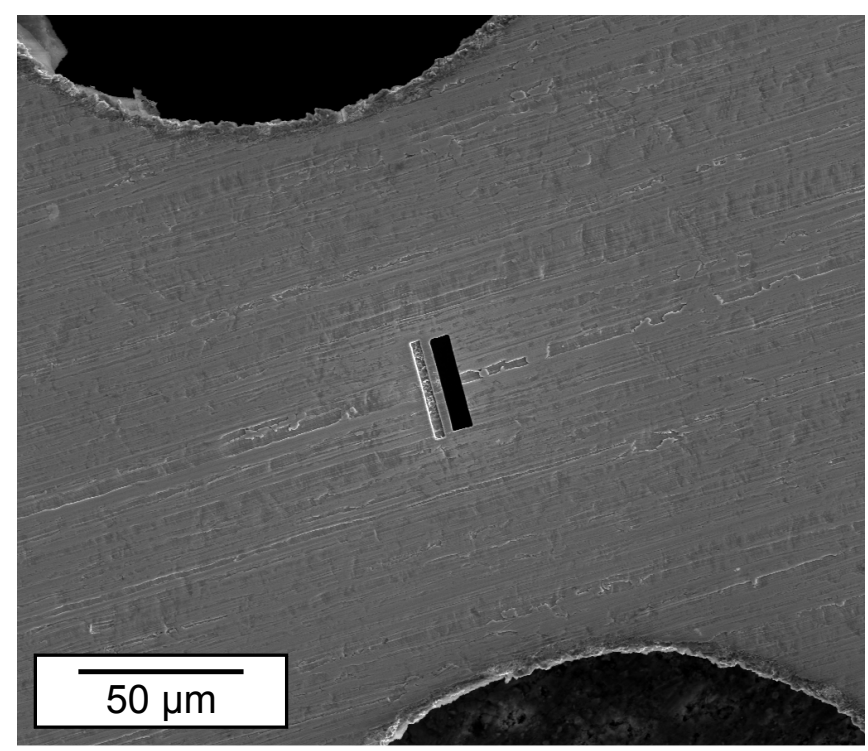

Abbildung 4.2: Vorbereiteter CuBe Rahmen - REM-Bild eines Rahmens, bei dem sowohl das Loch als auch eine Vertiefung für den Manipulator geschnitten wurde.

werden, dass keine plastische Verformung vor Beginn des Zugversuches stattgefunden hat.
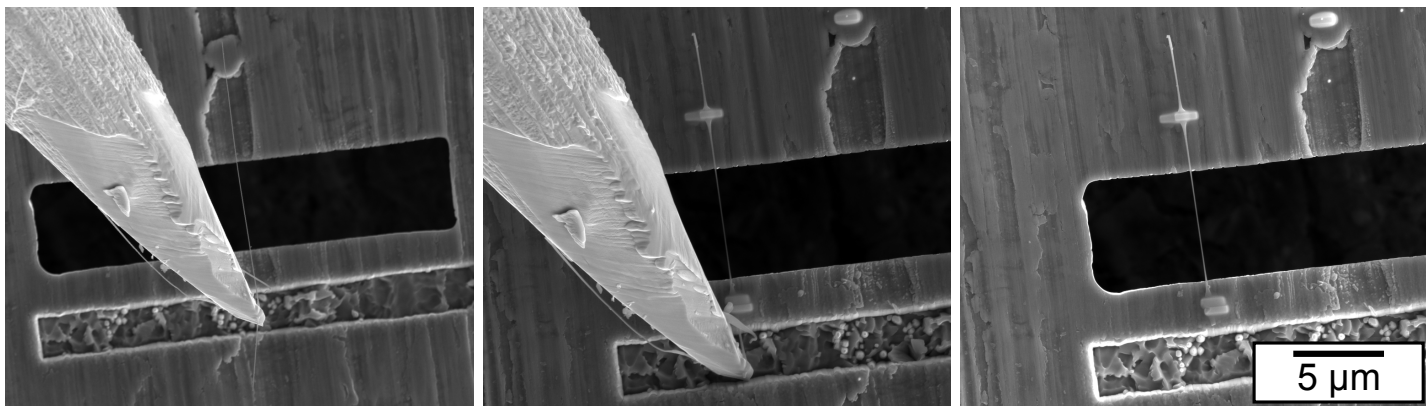

Abbildung 4.3: Prozessschritte zur Präparation eines Zugversuches - Auf den REM Bildern ist dargestellt, wie ein abgeholter Draht richtig ausgerichtet über der CuBe Struktur platziert (links) und befestigt (mitte) wird. Nach dem Entfernen des Mikromanipulators (rechts) ist der Transferprozess abgeschlossen.

\subsubsection{Co-Deformation}

Die Realisierung eines mechanischen Tests, bei dem die Probengröße im Bereich von einigen wenigen Mikrometern in der Länge liegt, ist experimentell herausfordernd. In 
den meisten Fällen sind Tests an solchen Proben daher nicht ohne entsprechende Hilfsmittel und eine angepasste Geometrie realisierbar. Ein Zugversuch als Methode zur Untersuchung von mechanischen Eigenschaften bildet dabei keine Ausnahme. So sind selbst bei guten Zugprüfanlagen die Verfahrschritte in der Größenordnung von 100 nm. Dies würde für einen $5 \mu \mathrm{m}$ langen Prüfkörper ein Dehnungsschritt von $2 \%$ entsprechen. Damit wäre eine genaue Überwachung der Verformung nicht möglich. Selbst Schrittgrößen von unter $5 \mathrm{~nm}$, wie sie der verwendete TEM-Zughalter der Firma Gatan bietet, liefern keine ausreichende Feinheit für den Test. Eine elegante und einfache Möglichkeit, die gegebene Schrittweite einer Apparatur deutlich zu verkleinern, bietet die Co-Deformation eines flexiblen Rahmens mit der zu untersuchenden Probe darauf. Das Prinzip eines derartigen Verfahrens soll im Folgenden genauer erklärt werden.

In Abbildung 4.4 ist eine schematische Zeichung des verwendeten Gatan Zughalters Model 654 dargestellt. Die Aufnahmepunkte für eine Probe liegen $9 \mathrm{~mm}$ auseinander. Vorgesehen ist zudem eine Befestigung der Probe mittels quadratischer Unterlegscheiben, die eine Verformung nur zwischen den 6,7 mm auseinanderliegenden Kanten zulässt. Eine Dehnung wird nun einseitig durch Verfahren des Fixierpunktes auf die

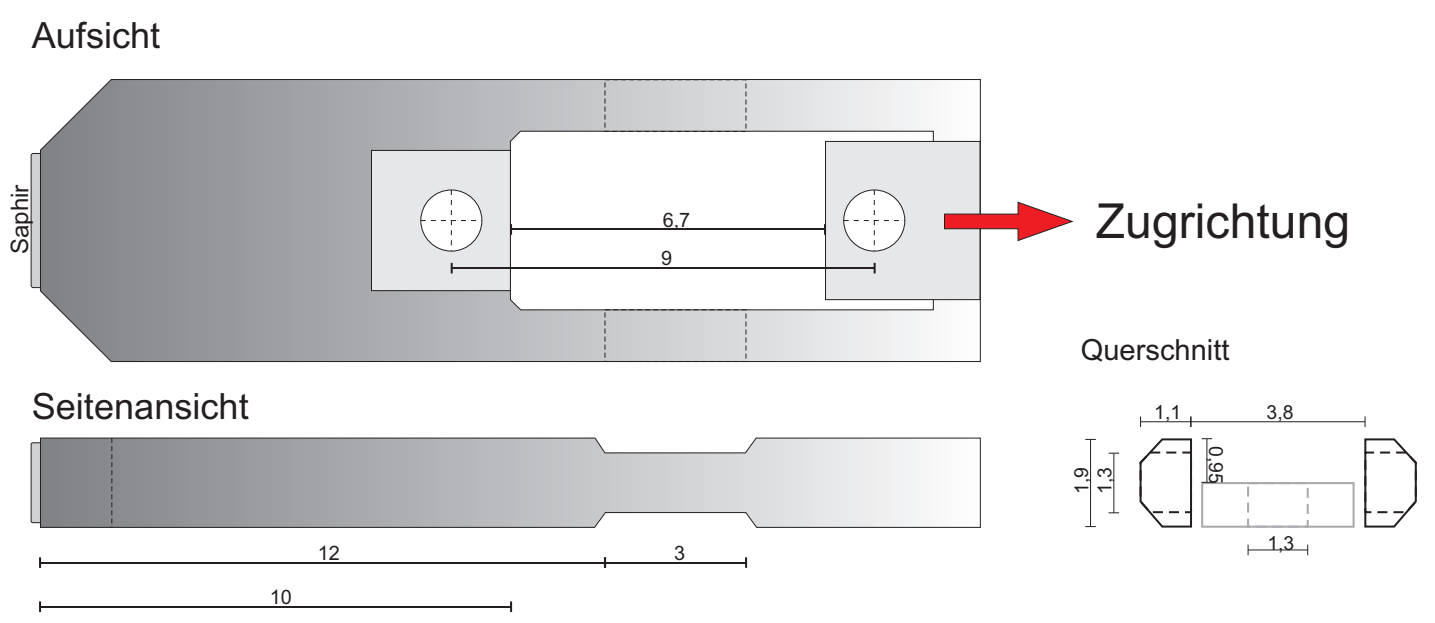

Abbildung 4.4: Zeichnung des Zughalters - Aufsicht auf die Probenaufnahme des verwendeten Zughalters Modell 654 der Firma Gatan. Die Bemaßung ist in mm angegeben.

Probenaufnahme gebracht. Die Schrittweite wurde in einem Vorexperiment ermittelt und beträgt etwa $5 \mathrm{~nm}$. Bezogen auf die Distanz zwischen den sich gegeneinander be- 


\section{EXPERIMENTELLE GRUNDLAGEN}

wegenden Aufnahmen des Halters ist dies eine Dehung von

$$
\varepsilon=\frac{5 \cdot 10^{-9} \mathrm{~m}}{6,7 \cdot 10^{-3} \mathrm{~m}}=7,5 \cdot 10^{-7} .
$$

Nimmt man nun an, dass sich das gesamte Material zwischen den Befestigungspunkten homogen verformt, folgt daraus, dass die Dehnung in jedem gedachten Teilstück der Dehnung der Gesamtprobenaufnahme entspricht. Legt man also seine eigentlich zu untersuchende Probe auf die Aufnahme, spürt sie einen deutlich verkleinerten Verformungsschritt im Vergleich zu den $5 \mathrm{~nm}$ der Direkteinspannung zwischen den Punkten. Die in den folgenden Seiten beschriebenen Versuche wurden mit einer etwas komplexeren Geometrie als für das obige Gedankenspiel durchgeführt. Eine Ansicht der verwendeten Rahmenstruktur ist in Abbildung 4.5 gezeigt. Der Rahmen ist aus einer

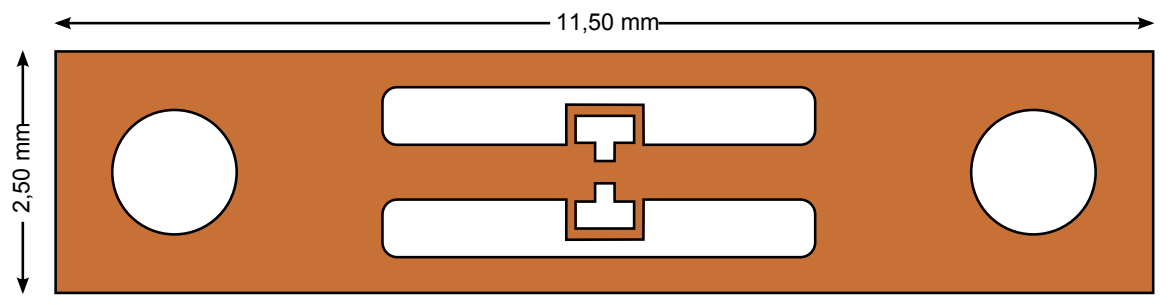

Abbildung 4.5: Schemazeichnung des CuBe-Rahmens - Dargestellt ist eine Zeichnung der Geometrie des CuBe-Rahmens. Zentral in der Mitte befindet sich der herstellerseitig gedünnte Bereich, in den für die elektronenmikroskopischen Untersuchungen das Loch geschnitten wurde.

Kupfer-Beryllium Legierung. Im mittleren Bereich der Struktur befindet sich ein Bereich, der auf eine Restdicke von etwa $30 \mu \mathrm{m}$ von der Rückseite gedünnt ist. Mit dem FIB wird dann ein rechteckiges Loch mit den Abmessungen von 5-6 $\mu \mathrm{m} \times 30 \mu \mathrm{m}$ von der Rückseite durch die Restdicke geschnitten. Dies liefert einen Loch, der mit dem TEM durchstrahlt werden kann. Der gedünnte Bereich ist es dann auch, der vornehmlich während des Zugversuches verformt. Um den Dehnungstransfer in dieser komplexen Struktur bestimmen zu können, wurde mit Hilfe von Finite Elemente Modellierung die Verformung simuliert [41].

In Abbildung 4.6 ist gezeigt, wie eine an den Befestigungspunkten angelegte Verformung auf den Draht transferiert wird. Der Verlauf zeigt im ersten Teil bis etwa $3,9 \mu \mathrm{m}$, dass der Draht unter kompressiver Spannung steht. Diese kann vor Beginn des Zugversuches als Verbiegung des Drahtes aus der Bildebene heraus im TEM beobachtet werden. 
In diesem Stadium findet jedoch keine plastische Verformung im Draht statt. Erst ab dem Zeitpunkt, an dem die Drahtverformung die Abzisse wieder von unten schneidet, beginnt der eigentliche Zugversuch (in der Grafik in rot schraffiert eingezeichnet). Aus

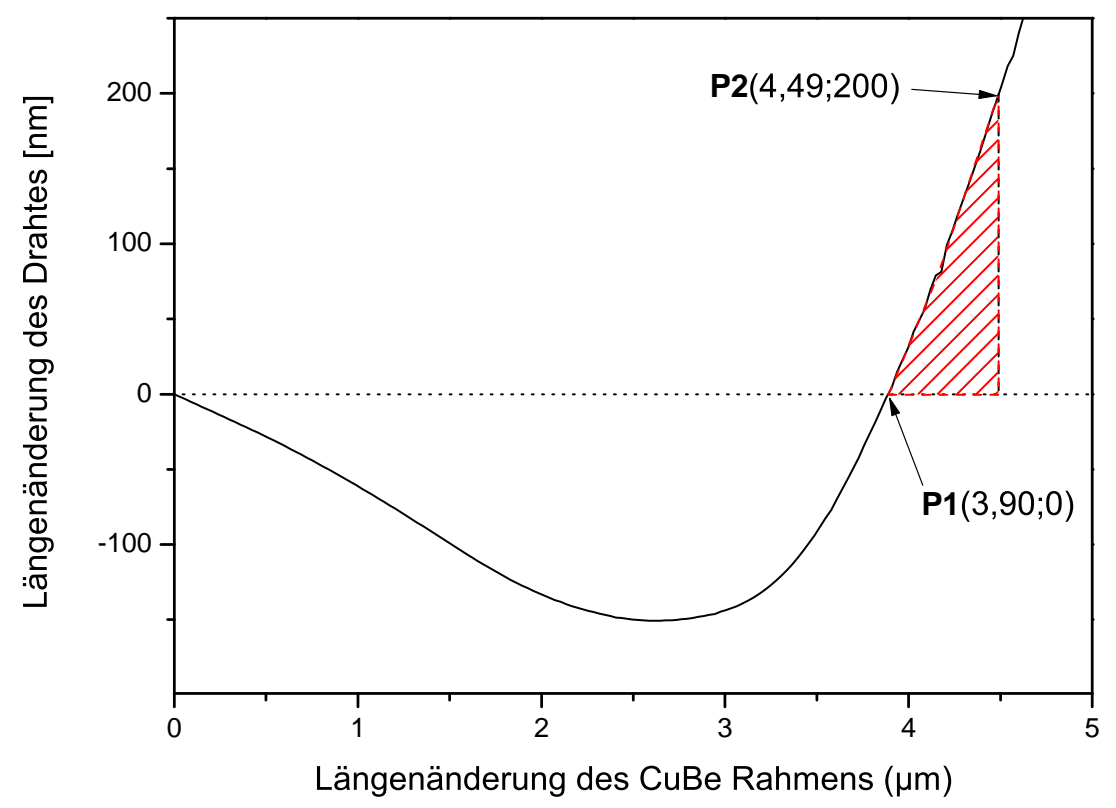

Abbildung 4.6: FE-Simulation der Verformung eines CuBe Rahmens - Längenänderung eines Drahtes, der mit Hilfe der CuBe-Rahmenstruktur verformt wurde. Auf der $\mathrm{x}$-Achse ist die Längenänderung der Rahmenstruktur zwischen den Befetigungspunkten dargestellt [41].

dieser Auftragung ist ablesbar, dass für eine Längenänderung des Drahtes um $200 \mathrm{~nm}$ (von P1 nach P2 in Abbildung 4.6) der Rahmen um etwa $600 \mathrm{~nm}$ gestreckt werden muss. Folglich ist eine Verkleinerung der Dehnung um den Faktor 3 erreicht worden. Für den Einzelschritt von $5 \mathrm{~nm}$ am Halter bedeutet dies eine Längenänderung des Drahtes von 1,67 nm. Dieser Wert entspricht in der Größenordnung etwa der Länge von 10 Burgersvektoren. Da während des Versuches jedoch das vereinzelte Auftreten von Defekten beobachtet werden konnte, erscheint dieser Wert etwas zu hoch. Erklärbar ist diese Diskrepanz durch zwei mögliche Aspekte. Zum einen ist vorstellbar, dass die Umsetzung der Rahmenstruktur in das elastische Modell der FE-Simulation nicht exakt genug war und daher eine größere Dehnungsskalierung als der Faktor 3 vorliegt. Zum anderen basiert die Bestimmung der Schrittweite am Halter auf einer Mittelung von 


\section{EXPERIMENTELLE GRUNDLAGEN}

einigen nacheinander ausgeführten Schritten. Befand sich darunter ein Verfahrschritt, der deutlich größer war als die durchschnittlich erwirkten Längenänderungen, hat dies einen direkt Einfluss auf die errechnete Schrittweite.

Zusammenfassend kann man sagen, dass die Co-Deformation mit der Rahmenstruktur durchaus sinnvoll erscheint, da sie zu einer Verkleinerung der Schrittweite führt. Eine exakte Bestimmung der Schrittgröße erweist sich zwar als schwierig, ist aber im Hinblick auf rein qualitative Experimente aber nicht von zentraler Bedeutung.

\subsubsection{Ablauf des Zugversuches}

Die in diesem Kapitel ausgewerteten Zugversuche an Gold Nanodrähten wurden alle unter vergleichbaren Bedingungen vorgenommen. Dazu wurde nach dem Transfer aus dem FIB (vgl. Abschnitt 4.2) der CuBe-Rahmen in den Verformungshalter eingesetzt. Vor Beginn des Tests ist jeweils im Hellfeldbild überprüft worden, ob Defekte im Draht vorliegen. Häufig beobachtet wurde hier bereits, dass vor Beginn des Zugversuches eine rein elastische Verbiegung das Drahtes vorlag. Dies war an Biegekontrasten entlang des Drahtes zu erkennen. Um für spätere Analysen die Geometrie möglichst einfach zu halten, war dann jeweils der nächste Schritt, den zu testenden Draht durch Verkippen des TEM-Halters in die Nähe oder direkt in eine niedrig indizierte Zonenachse zu bringen. In den meisten Fällen war dies eine [111] oder eine [110] Zonenachse. Welche Orientierung letztendlich bei den in diesem Kapitel gezeigten Ergebnissen vorlag, ist im Einzelfall angegeben.

Durch die Orientierung in eine Zonenachse war daraufhin die Bestimmung der Wachstumsrichtung möglich. Die Wachstumsrichtung der Drähte war bei allen durchgeführten Versuchen immer entlang der (110)-Richtung. Mit der Drahtachse war somit auch die Zugrichtung festgelegt und verlief dementsprechend ebenso entlang der (110)-Richtung. Sobald diese vorbereitenden Schritte abgeschlossen waren, wurde mit dem eigentlichen Zugversuch begonnen. Wie im Abschnitt über die Co-Deformation beschrieben wurde, war die kleinstmögliche Schrittweite des Halters etwa $5 \mathrm{~nm}$. Mit diesen Verformungsschritten wurde dann der Draht während des Zugversuches gedehnt. Bei einer Drahtlänge von etwa $5 \mu \mathrm{m}$ und einer Skalierung der Schrittweite durch die Rahmenstruktur um den Faktor 3 ergibt sich somit ein Dehnungsschritt der Größe

$$
\Delta \epsilon=\frac{1,67 \mathrm{~nm}}{5 \mu \mathrm{m}}=3,3 \cdot 10^{-4}=0,033 \% .
$$


Aus quantitativen Messungen [24] ist bekannt, dass die Dehnung beim Bruch etwa 3\% beträgt. Die Versuchsdauer betrug etwa 5 min, sodass der Draht mit einer mittleren Dehnrate von

$$
\dot{\epsilon}=\frac{3 \%}{300 \mathrm{~s}} \approx 1 \cdot 10^{-4} / \mathrm{s}
$$

verformt wurde.

Um die fortschreitende Verformung kontinuierlich aufzuzeichnen, wurden die Experimente mit der CCD Kamera gefilmt. Sobald der Videospeicher voll war, wurde eine Pause in der Verformung eingelegt und die gewonnenen Daten abgespeichert. Häufig wurden diese Pausen darüber hinaus verwendet, um detailreichere Bilder von dem Draht zu machen. Im Anschluss wurde der Draht in mehreren Schritten weiter bis zum Bruch verformt.

\subsubsection{Getestete Drähte im Überblick}

Tabelle 4.1 zeigt eine Zusammenstellung aller getesteten Drähte. Dabei zeigen die Spalten jeweils einige wichtige charakterisierende Parameter:

- Durchmesser des Drahtes, wie er im TEM erscheint

- Facettenlängen (wenn deren Auswertung möglich war)

- Verwendete Zonenachse während des Versuches

- Besonderheiten des Versuches.

Die Gesamtheit der getesteten Drähte in unterschiedlichen Belastungsgeometrien und ergänzende Informationen zu Verformungsverhalten und Orientierung im TEM während des Versuches sind im Anhang in Form einer Tabelle angegeben.

\subsubsection{Defektgeschwindigkeit}

Das Entstehen und die Bewegung von Defekten während der uniaxialen Verformung der Drähte geschieht auf einer so kurzen Zeitskala, dass diese nicht mit der zur Verfügung stehenden kürzesten Aufnahmedauer der CCD Kamera am TEM aufgelöst werden konnte. Die Bewegung von Versetzungen konnte also zu keinem Zeitpunkt direkt beobachtet werden. Daher ist es nur möglich eine Abschätzung für die minimale Geschwindigkeit anzugeben. Für die Auswertung dieser unteren Grenze der Geschwindigkeit von 


\begin{tabular}{|c|c|c|c|c|}
\hline Nr. & $\begin{array}{c}\text { Durchmesser } \\
{[\mathbf{n m}]}\end{array}$ & $\begin{array}{c}\text { Facetten } \\
(\text { lang/kurz) }[\mathrm{nm}]\end{array}$ & Zonenachse & Besonderheiten \\
\hline 1 & 80 & - & - & - \\
\hline 2 & 45 & - & {$[112]$} & \\
\hline 3 & 65 & $59 / 22$ & {$[110]$} & \\
\hline 4 & 50 & - & {$[110]$} & \\
\hline 5 & 40 & - & {$[111]$} & \\
\hline 6 & 60 & $38 / 35$ & {$[110]$} & \\
\hline 7 & 95 & $80 / 41$ & {$[111]$} & \\
\hline 8 & 90 & - & {$[110]$} & \\
\hline 9 & 200 & $200 / 20$ & {$[111]$} & \\
\hline 10 & 300 & $300 / x$ & {$[111]$} & \\
\hline 11 & 190 & $152 / 43$ & {$[111]$} & \\
\hline 12 & 65 & - & {$[111]$} & Blindversuch \\
\hline 13 & 100 & $76 / 45$ & [110] & Blindversuch \\
\hline 14 & 110 & & DT & SEM getesteter Draht \\
\hline 15 & 110 & $71 / 58$ & & \\
\hline 16 & 110 & $71 / 58$ & & \\
\hline 17 & 50 & - & {$[114]$} & Entlastungsversuch \\
\hline 18 & 180 & - & {$[112]$} & \\
\hline 19 & 170 & - & & SEM getesteter Draht \\
\hline 20 & 330 & $290 / 72$ & [111] & \\
\hline 21 & 60 & - & [110] & "Ga Draht" \\
\hline 22 & 125 & - & {$[112]$} & "Ga Draht" \\
\hline 23 & 240 & - & {$[100]$} & \\
\hline 24 & 220 & $270 / 45$ & {$[100]$} & \\
\hline 25 & 240 & - & {$[112]$} & \\
\hline 26 & 280 & $300 / 160$ & {$[100]$} & \\
\hline
\end{tabular}

Tabelle 4.1: Auflistung aller getesteten Gold-Drähte 
Partialversetzungen wird der größte Draht herangezogen, weil dort mit der längsten frei durchlaufbaren Wegstrecke zu rechnen ist. Diese Länge betrug bei dem so ausgewählten Draht 330 nm. Ausgehend von einer gleichmäßigen Bewegung der Versetzung innerhalb des Drahtes und einer Zeitaufösung der Kamera von 50 ms ist die untere Grenze gegeben durch

$$
v_{\text {mind }}=\frac{d}{t_{C C D}}=\frac{330 \cdot 10^{-9} \mathrm{~m}}{50 \cdot 10^{-3} \mathrm{~s}}=6,6 \cdot 10^{-6} \mathrm{~m} / \mathrm{s} .
$$

Die erwartete Geschwindigkeit für eine Versetzung ist abhängig von der aufgelösten Spannung $\tau$ auf dem Gleitsystem. Nach [7] und [42] kann diese ausgedrückt werden über:

$$
v=\frac{\tau}{B} \cdot e^{-\frac{20 \tau_{r e s i s t}}{\tau}}
$$

In [7] wurde die Berechnung für Gold durchgeführt mit dem Koeffizienten

$\mathrm{B} \approx 3 \cdot 10^{-5} \frac{\mathrm{Pa} \cdot \mathrm{s}}{\mathrm{m}}$. Der Reibungswiderstand $\tau_{\text {resist }}$ aufgrund der elastischen Ausbauchung einer Versetzung auf der Gleitebene beträgt von der Größenordnung in einem 100nm Draht etwa 100MPa [7]. Mit Hilfe dieser Werte lässt sich die Geschwindigkeit einer Versetzung bei gegebener Spannung berechnen. Nimmt man als Spannung einen Wert von $1 \mathrm{GPa}$ an, bei dem typischer Weise im Experiment die erste Nukleation beobachtet wird [24], erhält man eine Defektgeschwindigkeit von

$$
v=\frac{1 \cdot 10^{9} \mathrm{~Pa}}{3 \cdot 10^{5} \frac{P a \cdot s}{m}} \cdot e^{-\frac{20 \tau_{r e s i s t}}{1 \cdot 10^{9} P a}}=4,35 \cdot 10^{2} \mathrm{~m} / \mathrm{s}
$$

Da dieser Wert um einige Größenordnung über dem aufösbaren Wert liegt, wird klar, dass eine Zeitauflösung der Bewegung von Versetzungen in defektfreien Materialien mit der verwendeten Technik nicht realisierbar ist. Dieses Ergebnis ist insbesondere deshalb interessant, da ein Durchlaufen von vollen Versetzungen durch einen Draht vollkommen unbemerkt bleiben kann. Dies liegt daran, weil dieser Versetzungstyp außer einer Oberflächenstufe keine weitere Spur im Draht hinterlässt. Es bedarf also bei der Beurteilung der Ergebnisse einer sorgfältigen Abschätzung, inwieweit die beobachteten Defekte mit der ermittelten Dehnung übereinstimmen. Diese Rechnung wird ausführlich an einem Beispiel in Kapitel 5.1.4 durchgeführt. 


\section{EXPERIMENTELLE GRUNDLAGEN}

\subsection{Biegeversuche}

Als weitere Belastungsart an denselben Nanodrähten wurde ein Aufbau gewählt, der es ermöglichte, einen deutlich komplexeren Spannungszustand im Draht hervorzurufen. Dies wurde in einer Art Biegeversuch realisiert, bei dem der Draht zu Beginn entlang der Drahtachse gestaucht wurde. Durch das hohe Aspektverhältnis resultierte diese Belastung jedoch immer in eine Verbiegung, sodass der Spannungszustand, der letztendlich zu der Verformung führte, eine Mischung aus Biegespannung, Kompressionsspannung und Spannungsbeiträge durch eine Verdrillung des Drahtes war. Im weiteren Verlauf der Arbeit wird dieser Experimenttyp als Biegeversuch bezeichnet, da diese Beschreibung den Hauptaspekt der Belastung widerspiegelt und auch später zu Abschätzungen der wirkenden Spannungen herangezogen wird.

Zur Realisierung dieses Aufbaus wurde ein Standard lift-out Netzchen zur Befestigung von TEM-Lamellen der Firma Omniprobe vorbereitet. Im Wesentlichen bestand die Vorbereitung darin, ein Vertiefung im FIB zu schneiden, die das Eintauchen des Mikromanipulators vor dem Ablegen ermöglicht. Im Hinblick auf den verwendeten STMHalter im TEM musste die Vertiefung direkt von vorne erreichbar sein; daher war die Position an einem der beiden Stege des Netzchens prädestiniert. In Abbildung 4.7 ist ein so vorgeschnittenes Netzchen gezeigt. Die Drahtlänge für Biegeversuche ist deut-
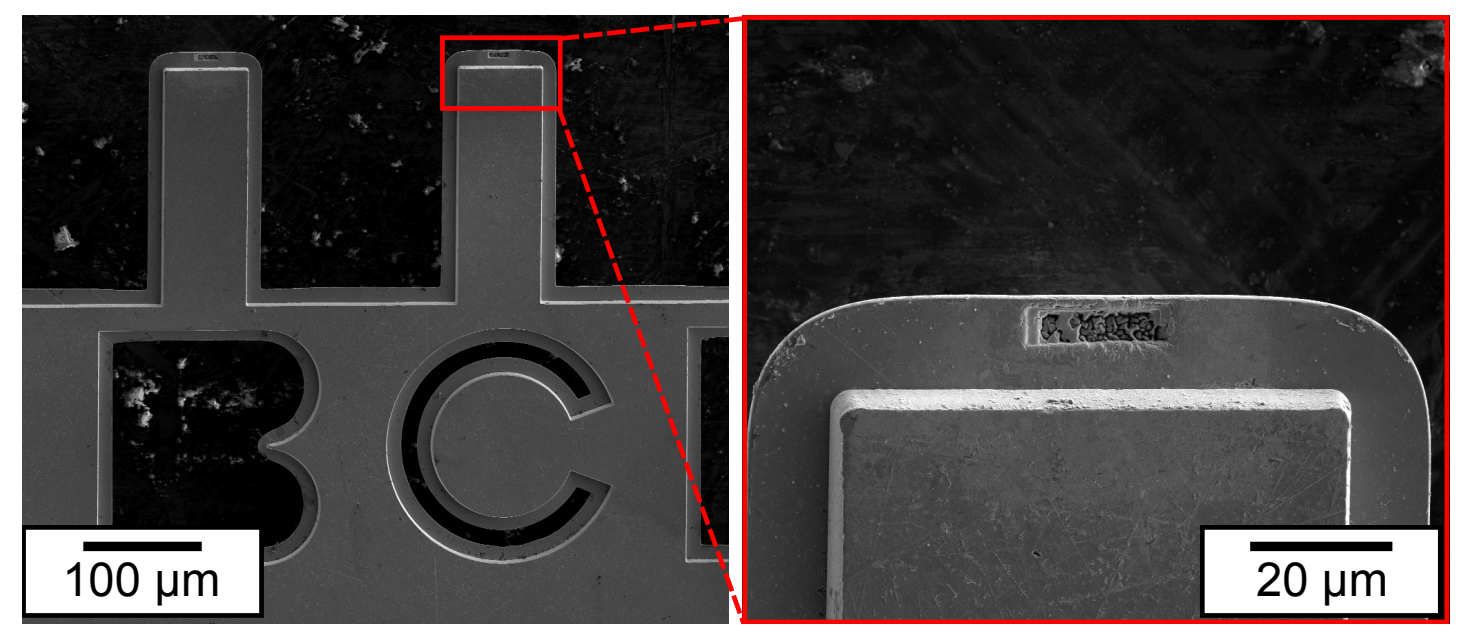

Abbildung 4.7: Vorgeschnittenes TEM-Netzchen für einen Biegeversuch - Die REM Aufnahmen zeigen das für einen Biegeversuch vorbereite Kupfer-Netzchen. An den mittleren Stegen des Omniprobe-grids sind Vertiefung für den Mikromanipulator geschnitten worden. 
lich kürzer als für Zugversuche. Um eine kontrollierte Verbiegung zu erreichen, wurde der Draht so gewählt, dass nur etwa $3 \mu \mathrm{m}$ aus dem Kupfer-Netzchen herausstanden. Das Ablegen wurde wiederum dadurch erreicht, dass der Tisch dem Draht entgegen angenähert wurde. Dabei dringt die Spitze der Omniprobe in das vorbereitete Fenster ein und der Draht kommt mit dem verbleibenden Steg in Kontakt. Ein Platinpflaster fixiert danach den Draht und die Omniprobe kann abgerissen und entfernt werden. Diese letzten Prozessschritte sind in Abbildung 4.8 dargestellt. Der Einbau dieser
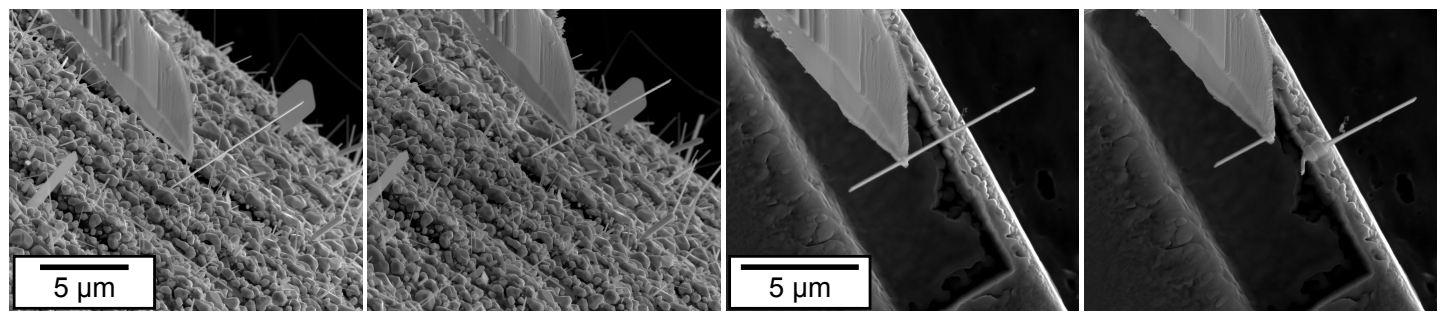

Abbildung 4.8: Präparation eines Biegeversuches - REM-Bilder von den letzten Prozessschritten für einen Biegeversuch.

so vorbereiteten Geometrie erfolgt danach im STM-Halter der Firma NanoFactory. Die wesentlichen Komponenten sind dabei die Klemmhalterung und der Kopf für die STM-Spitze. Das Kupfernetzchen wird mit Leitsilber in den Klemmhalter eingeklebt und dieser am Kupferdraht mit dem Halter verschraubt. In die Schraubaufnahme der Spitze wird eine elektrolytisch gedünnte Wolframnadel eingeführt und fixiert. Über den kugelförmigen Piezo-Kopf ist es möglich, die Spitze in die drei Raumrichtungen zu bewegen. Dabei gibt es zwei unterschiedliche Bewegungsmodi. Im Grobmodus wird eine Sägezahnspannung an den jeweiligen Piezo angelegt. Durch die Trägheit des Spitzenkopfes vollführt dieser dann eine so genannte "stick-slip"Bewegung, die den Kopf schrittweise in die gewünschte Richtung fährt. Im Feinmodus wird eine Gleichspannung an die Piezos angelegt, was zu einer zur Spannung proportionalen statischen Auslenkung führt. Der Bewegungsbereich im Feinmodus ist dabei auf $\pm 700 \mathrm{~nm}$ in jede Raumrichtung beschränkt. Eine Schemazeichung dieses Aufbaus ist in Abbildung 4.9 gezeigt. Die Verformung des Drahtes in dieser Geometrie erfolgt nun durch Bewegen der Spitze in Richtung des freistehenden Drahtendes. Sobald Kontakt besteht wird weiter axial bewegt bis sich der Draht verbiegt. Nach erfolgter Verformung können die entstandenen Defekte direkt in dem Halter untersucht werden. Weiterführende Untersuchungen wurde allerdings in einem herkömmlichen Doppelkipp-Halter durchgeführt 


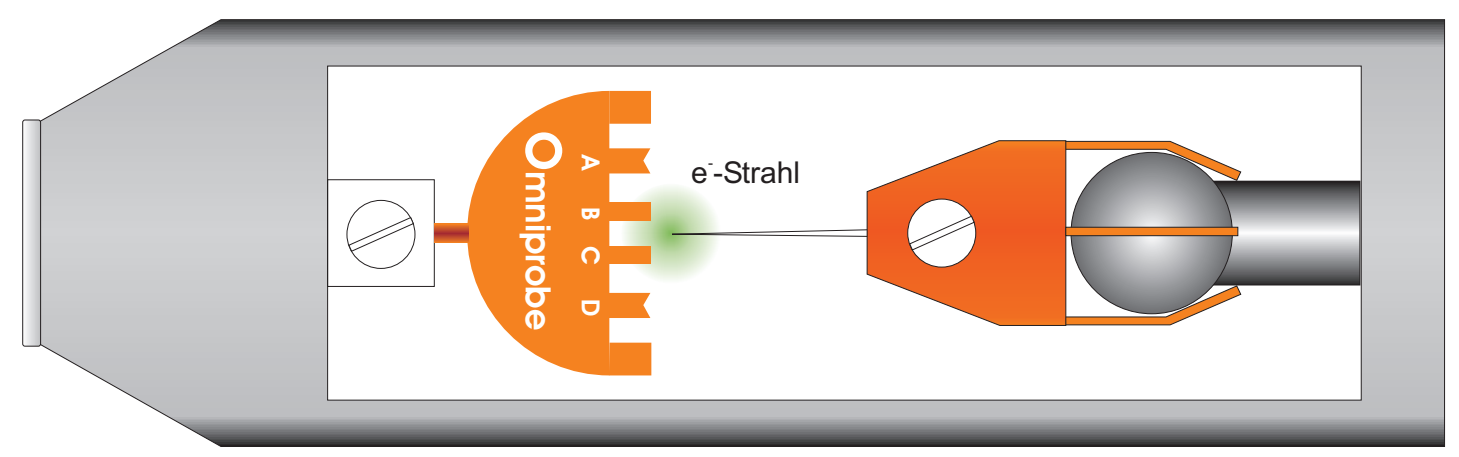

Abbildung 4.9: Schemazeichnung des STM-Halters - Zeichung des STM-Halter für das TEM mit eingebauter Probe und Wolframspitze auf dem beweglichen Piezo-Kopf.

indem das Kupfer-Netzchen wieder aus der Klemmhalterung herausgelöst wurde. Diese Verfahrensweise war von Vorteil, da in diesem Halter der Draht flexibler in alle Raumrichtungen orientiert werden konnte. Somit waren detailliertere Untersuchungen an den Defekten möglich. 
5

\section{Ergebnisse}

In diesem Kapitel sollen nun die experimentell gewonnenen Ergebnisse dargestellt werden. Es hat sich im Laufe der Versuche als sinnvoll erwiesen, diese Ergebnisse in zwei grundsätzlich verschiedene Kategorien aufzuteilen. Maßgeblich für die Einteilung in eine der beiden Kategorien ist dabei der während der Verformung auftretende Defekttyp. Die Aufteilung ist nötig, da die beiden beobachteten Defekttypen Ausdruck eines unterschiedlichen Verformungsmechanismusses sind und somit einer getrennten Behandlung bedürfen.

Einen Überblick über alle im Zugversuch getesteten Drähte wurde vorab schon in Abschnitt 4.2 gegeben und ist dort in Tabelle 4.1 zusammengefasst. Eine komplette Aufstellung aller verformten Drähte ist in einer Tabellle im Anhang gegeben.

\subsection{Zugversuche mit Stapelfehlern und Zwillingen}

Im ersten Abschnitt dieses Kapitel soll das Verformungsverhalten von Drähten erläutert werden, deren typischer Defekttyp, der während der Verformung beobachtet werden kann, Stapelfehler und Zwillinge sind. Die Rechtfertigung dieser Aussage folgt aus detaillierten Analysen des Beugungsbildes und quantitativen Untersuchungen im TEM. Sie wird im Laufe dieses Kapitels gegeben, nachdem allgemeinere Aussagen über die Defektentwicklung und deren Verteilung untersucht wurden. Neben der Beschreibung des Defekttyps und der räumliche Verteilung der Defekte wird noch auf das Bruchverhalten eingegangen. Durch ergänzende Kontrollexperimente wird in den letzten Abschnitten die Grundlage gelegt einige wichtige Parameter besser diskutieren zu können. 


\subsubsection{Verlauf der Defektdichte}

Ausgehend von einem defektfreien Draht nach dem Transfer auf den CuBe-Rahmen (vgl hierzu auch 4.2.2) nimmt mit Beginn der plastischen Verformung der Probe die Defektdichte zu. Der genaue zeitliche Verlauf der Defektanzahl konnte an einem Draht sehr gut nachvollzogen werden. Dies war möglich, da über den gesamten Versuch der gleiche Abschnitt des Drahtes im sichtbaren Bereich der Kamera verblieb. Für die Abbildung 5.1 wurde jede Kontraständerung zwischen zwei Einzelbildern der Videodateien als das Auftreten eines Defektes interpretiert und gezählt. Zu erkennen ist also das Ansteigen der Defektzahl mit zunehmender Zeit. Da alle Videos mit konstanter Bildfrequenz aufgezeichnet wurden kann die gewählte Auftragung gegen die Bildzahl auch als Zeitskala interpretiert werden. In der Zeit zwischen den Videoteilen (die Bereiche eines Videoteils werden repräsentiert durch die vertikalen gestrichelten Linien) wurden Umstellungen an der Kamera vorgenommen, um hochauflösende Bilder vom Verformungsstand und den entstandenen Defekten zu machen. Ausserdem wurden die Einzelbilder des Videos abgespeichert. Auffallend in Abbildung 5.1 ist, dass nach Ende eines Videoteiles die Defektanzahl für eine gewisse Zeit konstant bleibt, obwohl am Zughalter die schrittweise Verformung direkt nach Beginn der Aufnahme fortgeführt wird. Eine mögliche Erklärung für diese Beobachtung ist, dass in der Zeit zwischen den Videoteilen eine Relaxation im CuBe-Rahmen stattfindet und das Spannungsniveau am Draht dadurch abnimmt. Dieses muss dann beim Wiederaufnehmen der Verformung am Halter erst wieder neu aufgebaut werden, um eine weitere plastische Verformung im Draht hervorzurufen. In dieser verstrichenen Zeit bis zum weiteren Verformen werden somit keine weiteren Defekte erzeugt und die Zahl in Abbildung 5.1 bleibt konstant.

Die unterschiedlichen Steigungen in der Defektzahlentwicklung sind mit unterschiedlichen Dehnungsgeschwindigkeiten zu erklären. Da die Verformungsgeschwindigkeit nicht am Halter direkt eingestellt werden kann, sondern durch das schrittweise Verfahren bestimmt wird, sind Abweichungen zwischen den Verformungsabschnitten möglich. Darüber hinaus ist denkbar, dass die Verformung, wie sie in Abschnitt 4.2 angenommen wurde, nicht linear auf eine äußere Verlängerung reagiert und der Draht dadurch effektiv schneller gedehnt wird.

Der Beginn der plastischen Verformung wird gekennzeichnet durch das vereinzelte Ent- 


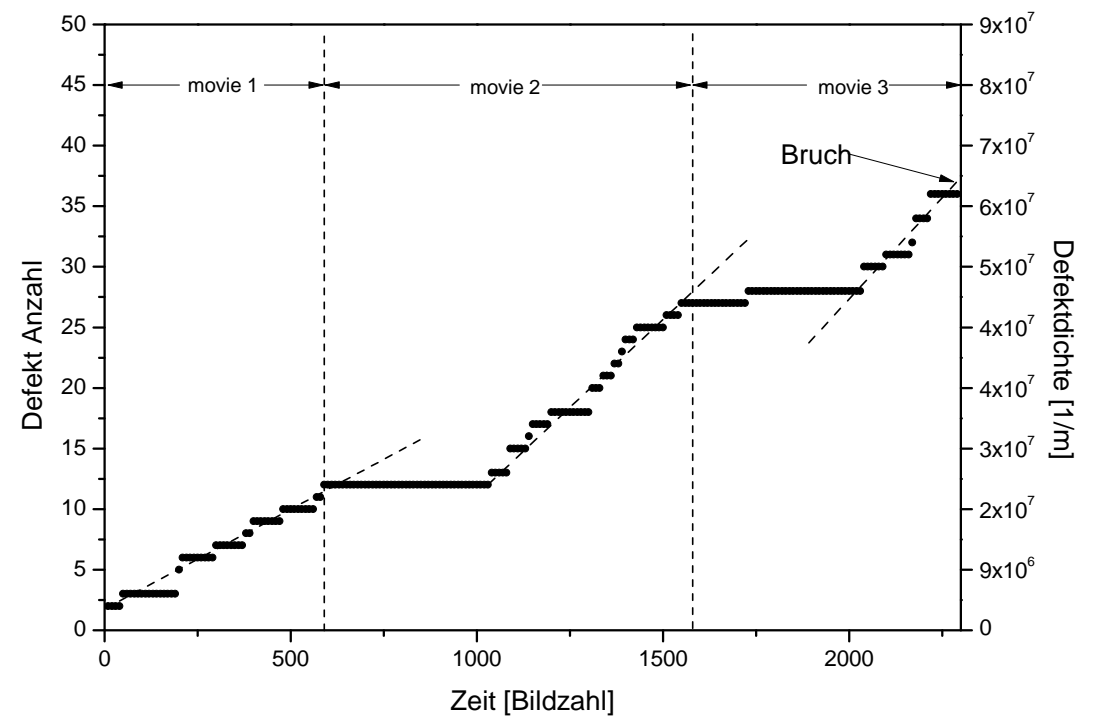

Abbildung 5.1: Entwicklung der Defektanzahl - Ermittelte Anzahl von Defekten in einem $55 \mathrm{~nm}$ breiten Draht, die im gesamten Verlaufe eines Zugversuches bis zum Reissen entstehen. Die gestrichelten Linien weisen auf eine Unterbrechung des Zugversuches hin.

stehen von planaren Defekten. Dies geschieht an zum Teil weit voneinander liegenden Punkten des Drahtes. Abbildung 5.2 zeigt zum Beispiel zwei Einzelbilder aus einem Zugversuch, auf denen die drei ersten beobachteten Defekte zu sehen sind. In diesem Fall ist erkennbar, dass der Abstand zwischen dem ersten und zweiten Defekt $250 \mathrm{~nm}$ beträgt. Darüber hinaus erfolgt die Nukleation anfänglich offenbar nur auf einer Ebene. Mit zunehmender Verformung verringert sich der mittlere Abstand der Defekte allerdings weiter. Weiterhin kann beobachtet werden, dass Defekte auch mit einer anderen Orientierung im Draht liegen. In dem oben angeführten Beispiel war dies der Fall für den fünften zählbaren Kontrast. Diese anderen Kontraste liegen in einem festen Winkel zur Lage der zuerst aufgetretenen Defekte. Außer diesen beiden Orientierungen tritt keine andere abweichende Orientierung bei allen weiteren Defekten auf. In seltenen Fällen kann beobachtet werden, dass der Kontrast von planaren Defekten wieder verschwindet (in 5.3 ist ein solcher Fall gezeigt). Zu dieser Phase der Verformung ist im Beugungsbild des verformten Drahtes bereits eine Veränderung an den Beugungspunkten beobachtbar. Es kann dort eine Streifenbildung in bestimmte Richtungen beobachtet werden. Dies liefert einen Hinweis auf nicht fcc koordinierte Lamellen, die sich innerhalb des 


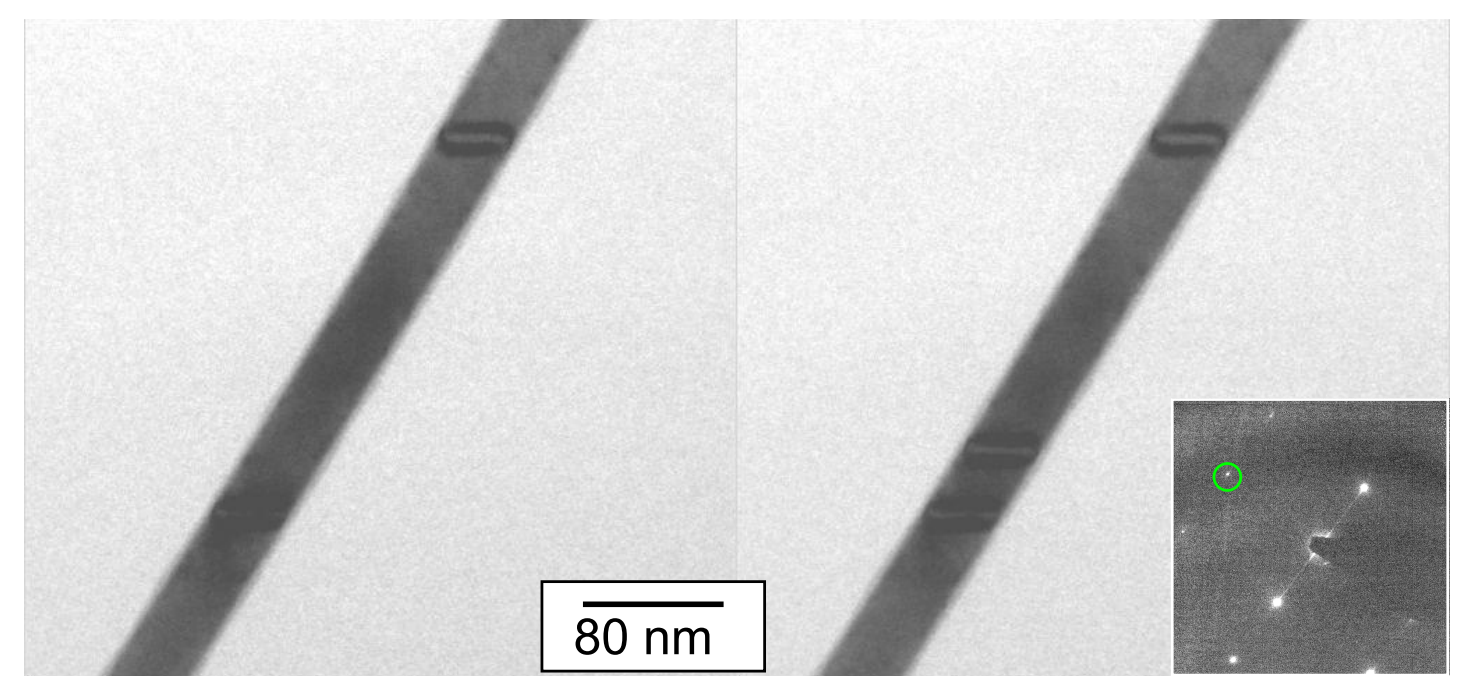

Abbildung 5.2: Beginn der plastischen Verformung während des Zugversuches - Hellfeldaufnahme der ersten vier unabhängig voneinander entstandenen planaren Defekte in einem $65 \mathrm{~nm}$ Draht

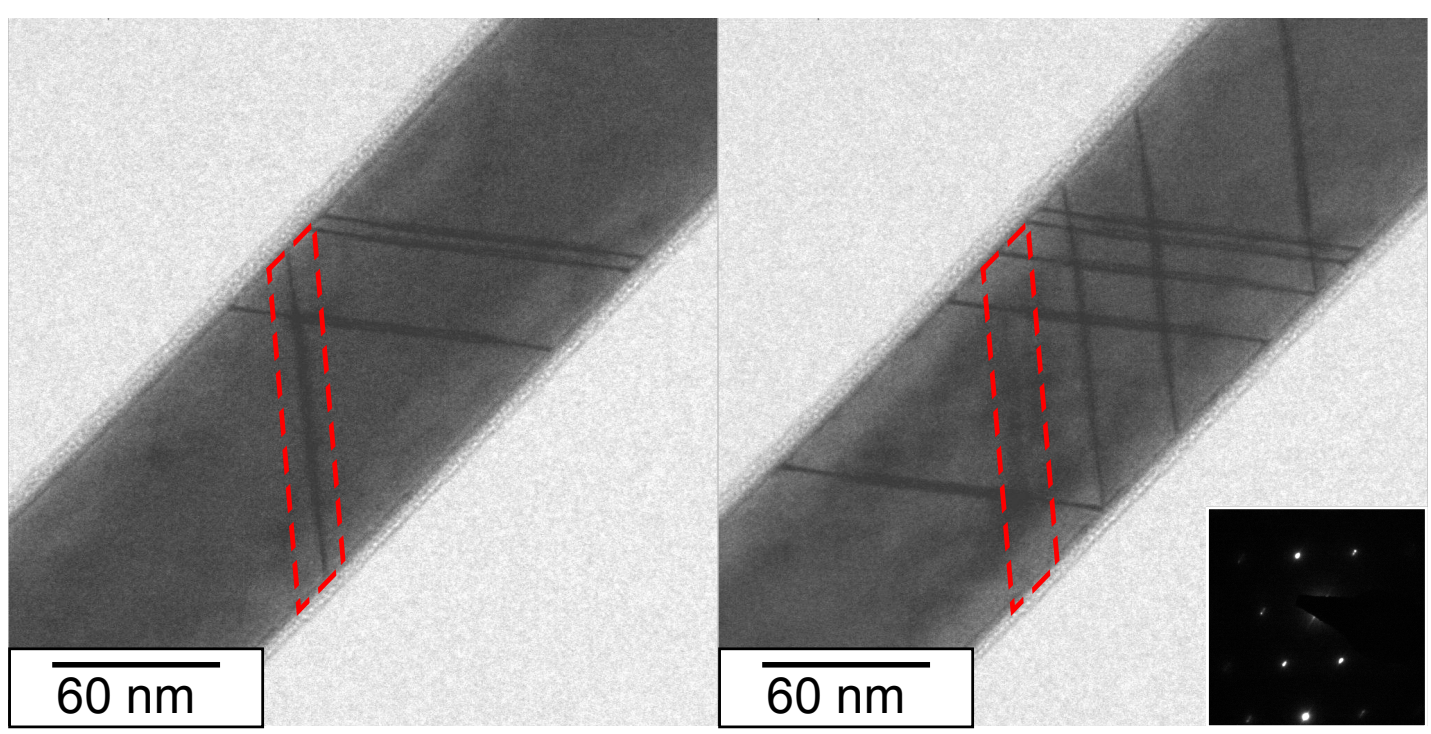

Abbildung 5.3: Fortschreitende Verformung - Höhere Verformung mit zwei unterschiedlichen Orientierungen von planaren Defekten in einem $95 \mathrm{~nm}$ Draht. Erkennbar ist an diesen Einzelbildern auch, dass ein Kontrast wieder verschwindet, während fünf neue gebildet werden. 
Materials befinden (vgl dazu auch Kapitel 2.2).

Mit weiterer Dehnung des Drahtes nimmt der mittlere Abstand der Stapelfehler weiter ab, gleichzeitig kann im Beugungsbild das Auftreten von Zwillingen im Draht nachvollzogen werden. Im Hellfeldbild ist es hingegen kaum möglich, sehr dünne verzwillingte Bereiche von anderen planaren Defekten sicher zu unterscheiden.

Bedingt durch die zunehmende Defektdichte und das Auftreten von zwei Orientierungen ist es irgendwann nicht mehr möglich diese unabhängig in ungestörte Bereiche des Drahtes zu platzieren. Daher kommt es im Laufe des Zugversuches zum Durchdringen der Defekte. Ein Beispiel von sich kreuzenden Defekten ist in Abbildung 5.3 gezeigt. Aus der Auftragung in Grafik 5.1 ist zu entnehmen, dass die Defektdichte bis zum Bruch linear ansteigt, die maximale Defektdichte wird somit kurz vor dem Reissen des Drahtes erreicht. Abbildung 5.4 zeigt die Situation des verformten Drahtes im letzten Einzel-

\section{$120 \mathrm{~nm}$}

Abbildung 5.4: Maximale Defektdichte kurz vor Reissen des Drahtes - Hellfeldaufnahme von planaren Defekten und Zwillingen in einem Draht mit einem Durchmesser von $55 \mathrm{~nm}$

bild des Videos bevor es zum Bruch kommt. In dem dort gezeigten Fall ist durch die gewählte Ausrichtung des Drahtes relativ zum Elektronenstrahl nur die Beobachtung von Stapelfehlern auf einer der beiden auftretenden Orientierungen möglich. Die ermittelte Anzahl der linienhaft erscheinenden Kontraste ist dort 36. Auf einer betrachteten Länge von $560 \mathrm{~nm}$ ergibt sich damit also ein mittlerer Abstand

$$
\bar{d}=\frac{560 \cdot 10^{-9} \mathrm{~m}}{36}=15,6 \mathrm{~nm} .
$$

Nimmt man eine Gleichverteilung der Defekte auf die beiden beobachtbaren Orientierungen an, halbiert sich entsprechend der mittlere Abstand zu

$$
\bar{d}=\frac{560 \cdot 10^{-9} \mathrm{~m}}{72}=7,8 \mathrm{~nm} .
$$




\section{ERGEBNISSE}

Die Auflösung des Einzelbildes innerhalb eines Videos beträgt in der obigen Vergrößerungsstufe 1 Pixel pro Nanometer. Mit Kenntnis dieses Wertes kann die obige Rechnung als Abschätzung für eine untere Grenze der Defektdichte verstanden werden. Dies liegt daran, da es ohne weiteres möglich ist, dass ein gezählter Kontrast ein größeres Volumen besitzt und eigentlich durch mehrere benachbarte Defekte gebildet wird. Die Klärung dieser Frage ist allerdings mit der hier verwendeten Technik nicht möglich und wäre nur über hochauflösende Mikroskopie zu erreichen.

Die räumliche Verteilung der einzelnen planaren Defekte wurde für die maximaler Defektdichte im letzten Einzelbild vor dem Reissen evaluiert. Dazu wurde bei jedem Pixel einer Linie entlang der Drahtachse entschieden, ob ein Defekt erkennbar ist oder nicht. Dies liefert eine binäre Information über die Verteilung der Defekte. Das Ergebnis dieser Auswertung ist vergleichend in Abbildung 5.5 dargestellt. Zur weitergehenden

\section{Stapelfehler Verteilung}

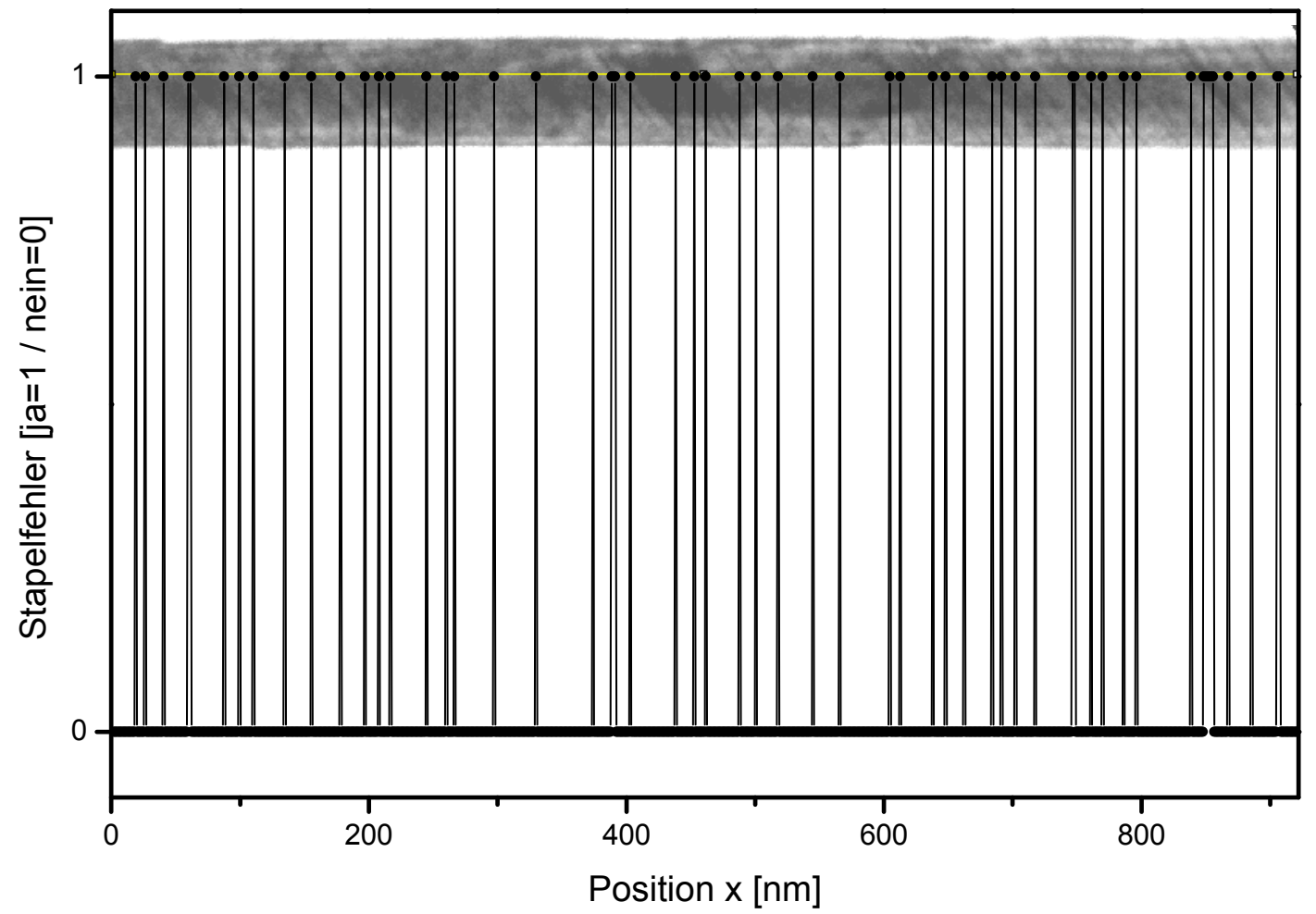

Abbildung 5.5: Defektverteilung - Binäre Information über die Lage der Defekte in einem 100 nm Draht kurz vor Ende des Versuches 
Auswertung ist der jeweilige Abstand zwischen zwei benachbarten Defekten betrachtet worden. Damit soll die Frage untersucht werden, wie die Verteilung der Defekte auf die zur Verfügung stehende Drahtlänge geschieht. Auskunft über diesen Sachverhalt liefert ein Histogramm der Abstände.

Durch die diskrete Verteilung auf Pixel in dem Ausgangsbild liefert die Zählung der Abstände eine erhöhte Häufigkeit beim Abstand von 0,901 nm, was der Länge eines Pixels entspricht. Dies liegt in der Behandlung von geschlossenen Kontrastbereichen innerhalb des Drahtes begründet. Liegt ein solcher Bereich vor, werden alle benachbarten Bildpunkte bis zum Ende des Kontrastes gezählt. Damit erhält man häufig den Abstand von genau einem Pixel.

Für die Untersuchung der Verteilung wurden Abstandsklassen mit einer Breite von 5 $\mathrm{nm}$ eingeführt, die symmetrisch um 2,5 nm (d.h. $0 \mathrm{~nm}$ bis $5 \mathrm{~nm}$ ) beginnen. Das Ergebnis der so gewonnenen Abstandsverteilung ist in Form eines Histogramms in Abbildung 5.6 gezeigt. Mit einer derartigen Auftragung ist es möglich, die Abstandsverteilung mit einer statistisch erwarteten Verteilungen zu vergleichen. Auf die Interpretation dieser Verteilung wird in Abschnitt 6 im Detail eingegangen.

\subsubsection{Analyse der Stapelfehler}

Die Frage nach der genauen Natur der beobachteten Defekte in den vorher beschriebenen Zugversuchen blieb bislang ungeklärt. Die Unterbrechungen während der Zugversuche und ergänzende quantitative Analysen wurden daher herangezogen, um die Defektart im TEM genau zu bestimmen. Die Tatsache, dass die Defekte nur in zwei Orientierungen in dem Draht vorlagen und kein Spannungsfeld mit ihnen direkt verknüpft war, lässt den Schluss zu, dass es sich um planare Defekte auf kristallographischen Ebenen handelt. Die gemessenen Winkel in Bildern wie Abbildung 5.3 ergeben etwa $\pm 53,5^{\circ}$ relativ zur Drahtachse. Dies liegt sehr nahe am Winkel von $54,7^{\circ}$ zwischen einer $\{111\}$-Ebene in kubisch flächenzentrierten Materialien und der Drahtachse, die entlang $<110>$ liegt.

Wie im Kapitel 2.2 bereits beschrieben, kann ein Stapelfehler auf einer $\{111\}$-Ebene in kubisch flächenzentrierten Materialien als eine dünne Lamelle mit hexagonal dichtest gepackter Koordination verstanden werden. Dies hat im Beugungsbild zur Folge, dass aufgrund der eingeschränkten Dicke der Lamelle die Beugungsreflexe in die zu 


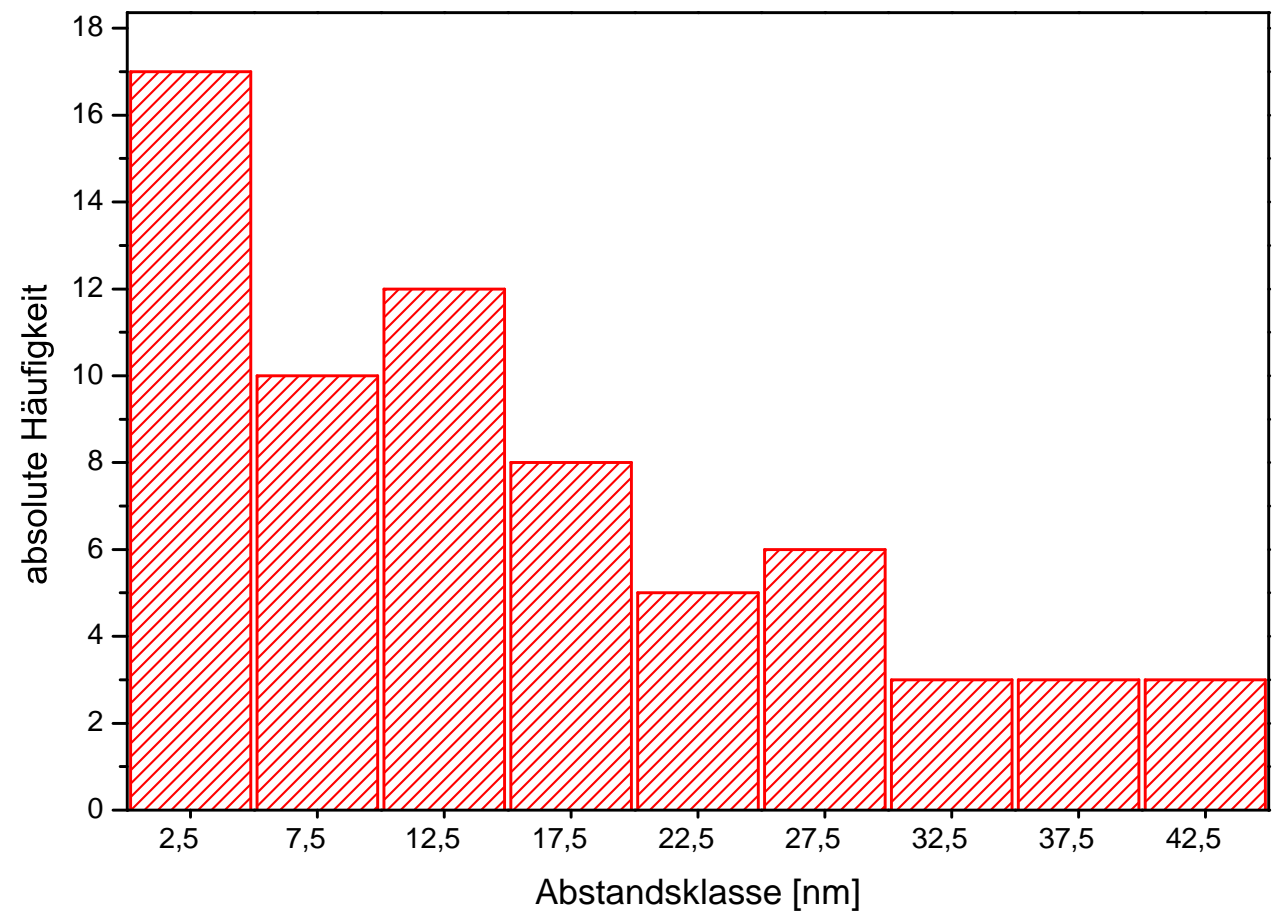

Abbildung 5.6: Abstandsverteilung der Stapelfehler - Die Auftragung zeigt die Anzahl der gezählten Defekte aus der Binarisierung zusammengefasst in $5 \mathrm{~nm}$ großen Abstandsklassen. 
der Defektebene gehörige $\{111\}$-Richtung verlängert werden. Dieses sogenannte "streaking" ist in Abbildung 5.7 gezeigt und war in allen [110]-Zonenachsen während der Verformung deutlich sichtbar. Mit der so durchgeführten Analyse des Beugungsbildes

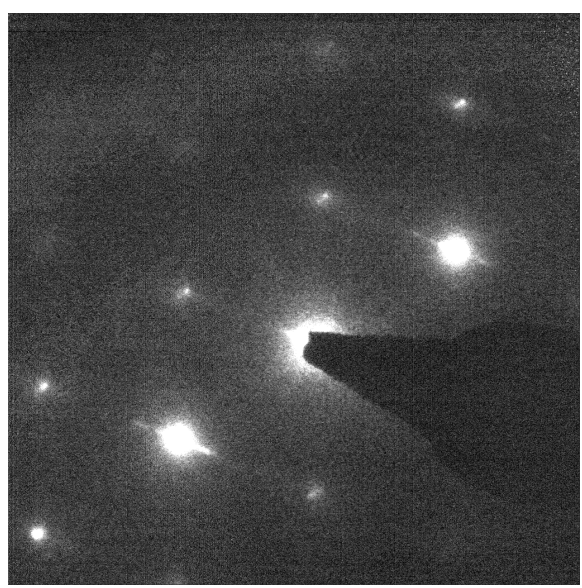

Abbildung 5.7: „Streaking“ der Beugungsreflexe durch Stapelfehler - [110]Zonenachse, die die Aufweitung von Beugungsreflexen in die [1111]-Richtung zeigt. Dies ist die Richtung des Normalenvektors der hcp Lamelle.

ist man in der Lage zu bestimmen, ob ein planarer Defekt vorliegt und auf welcher Ebene sich dieser befindet. Über den genau vorliegenden Typus erhält man allerdings keine weiteren Informationen. Um diese weitergehende Information zu erhalten bedarf, es einer quantitativen Kontrastanalyse im TEM.

An einem Draht wurde eine solche quantitative Defektanalyse durchgeführt, um den Translationsvektor des vorliegenden planaren Defektes zu ermitteln. Dazu wurde ein getesteter Draht unter Zuhilfenahme der Manipulationstechniken aus Abschnitt 4.1 auf ein Omniprobe TEM Halbnetz transferiert. Im Doppelkipphalter wurden dann in 3 verschiedenen Zonenachsen 12 Bilder gemacht (vgl. Abbildung 5.8), die den Stapelfehler in unterschiedlichen Abbildebedingungen zeigen. Aus der Kombination der SchwarzWeiß-Information der oszillierenden Kontraste und dem Vergleich mit dem errechneten Kontrastverlauf für mögliche Stapelfehlertypen ist dann eine Aussage über den Translationsvektor möglich.

In dem untersuchten Fall lag der Translationsvektor entgegengesetzt zur Einfallsrichtung des Elektronenstrahls. Mit der Kenntnis der Lage der betrachteten \{111\}-Ebene ist der Schluss zulässig, dass es sich bei dem analysierten Stapelfehler um einen planaren Defekt von extrinsischer Natur handelt. 


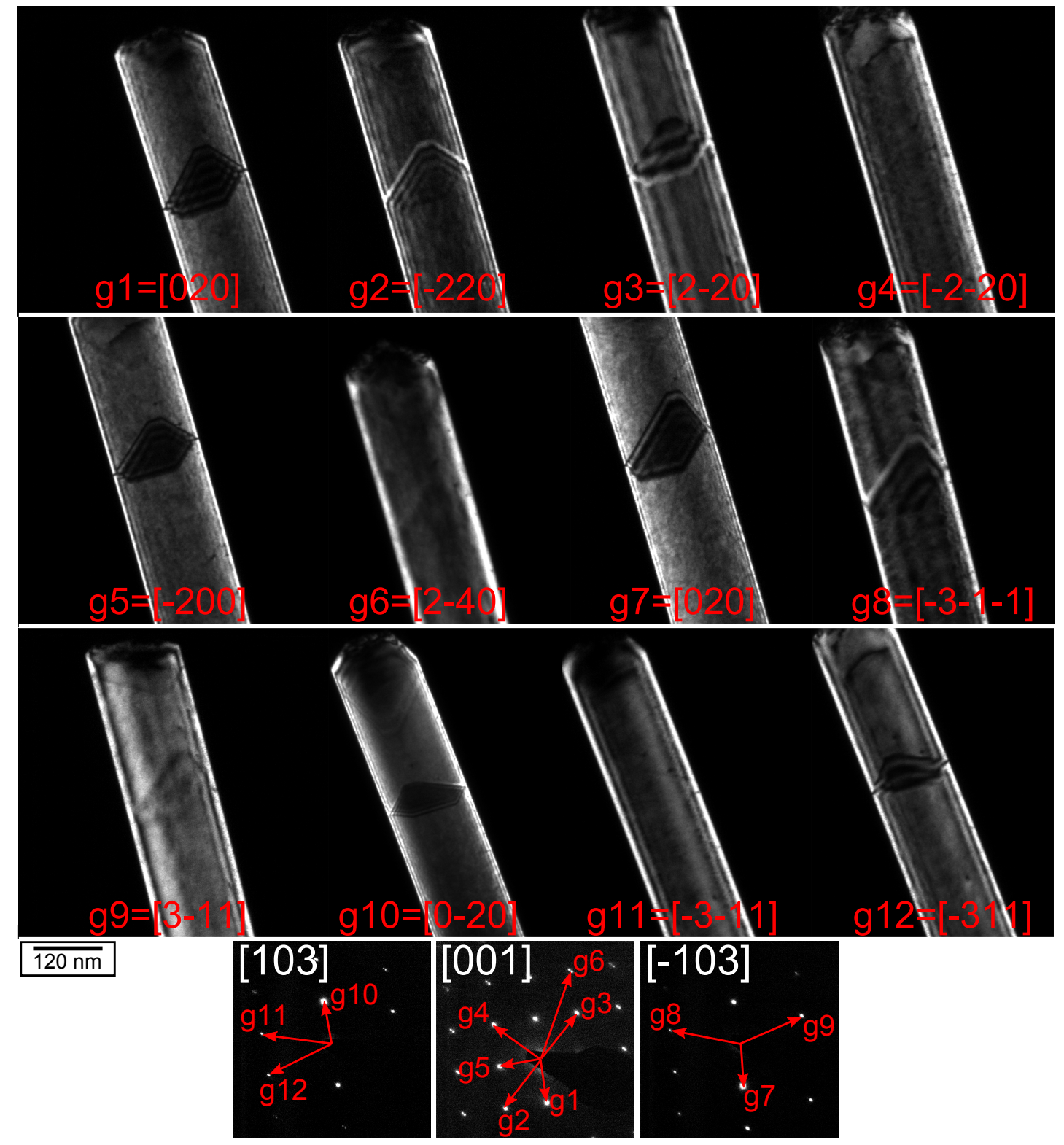

Abbildung 5.8: Quantitative Analyse eines Stapelfehlers - Die Dunkelfeldbilder zeigen denselben Stapelfehler in unterschiedlichen Abbildebedingungen. Aus den Kontrastvariationen lässt sich der Typ des Stapelfehlers identifizieren. Im hier gezeigten Beispiel handelt es sich um einen extrinisichen Stapelfehler. 
Die Form, in der ein planarer Defekt wie ein Stapelfehler im TEM erscheint, hängt von unterschiedlichen Faktoren ab. Grundsätzlich entscheidet die Lage relativ zum Elektronenstrahl und damit der gewählte Abbildevektor, ob der Defekt überhaupt sichtbar ist oder nicht. Ist die Sichtbarkeit sichergestellt, repräsentiert der flächenhafte Defekt die Querschnittsfläche auf der entsprechenden Ebene. Besonders deutlich wird dieser Effekt, wenn eine nicht triviale Form, wie zum Beispiel ein unregelmäßiges Sechseck, vorliegt. Ein Beispiel von Querschnitten und projizierten Stapelfehlerformen ist in Abbildung 5.9 gezeigt.

Aus den gewonnenen Erkenntnissen über den beobachteten Defekttyp lassen sich nun

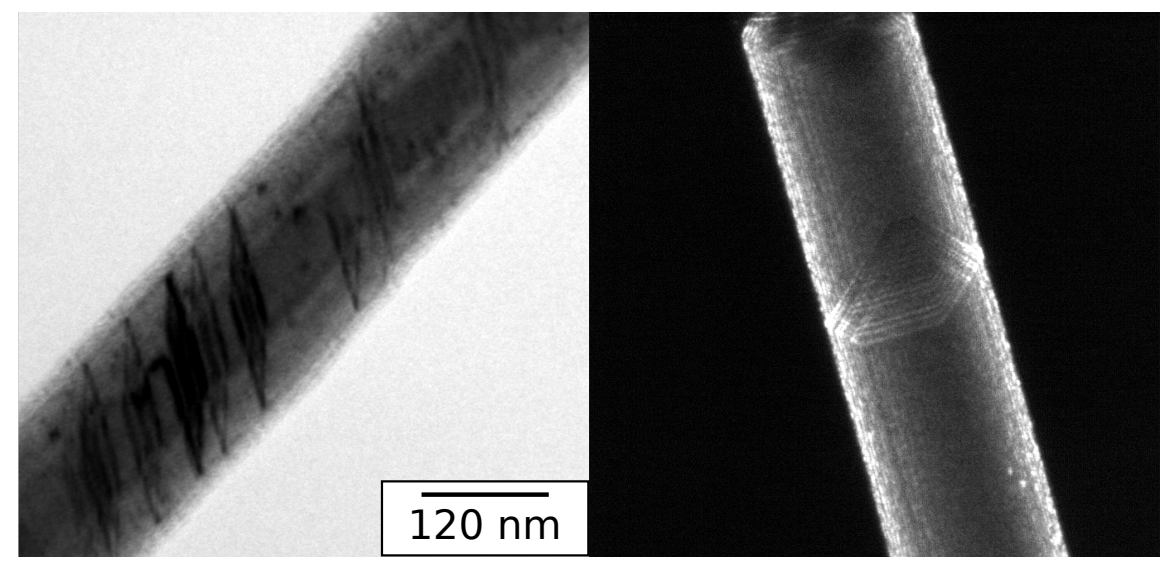

Abbildung 5.9: Planare Defekte, die die Querschnittsform des Drahtes wiedergeben - Wird ein planarer Defekt auf einer $\{111\}$-Ebene durch die Facetten des Drahtes begrenzt, gibt der im TEM beobachtete Kontrast die Querschnittsfläche des Drahtes auf eben dieser Ebene wieder. Die beiden Beispiele zeigen zwei verschiedene Drahtquerschnitte.

Rückschlüsse auf den Verformungsvorgang ziehen. Eine gängige Erklärung für das Auftreten von Stapelfehlern während der Verformung ist die Bewegung von Partialversetzung. Diese hinterlassen beim Durchlaufen des Kristalls ein gestörtes Gitter. Die kristallographischen Beziehungen wurden hinreichend in Kapitel 2.1.1 erläutert. Abschließend lässt sich also sagen, dass während keines Zugversuches Partialversetzungen direkt beobachtet werden konnten, sondern vielmehr die hinterlassenen Stapelfehler als Spur der Bewegung dieser Partialversetzungen interpretiert wird. 


\title{
5.1.2.1 Interaktion von Stapelfehlern
}

Die Beobachtung der Verformung der Drähte hat gezeigt, dass die Stapelfehler auf zwei verschiedenen $\{111\}$-Ebenen vorzufinden sind. Dies bedeutet, dass sich die Stapel-

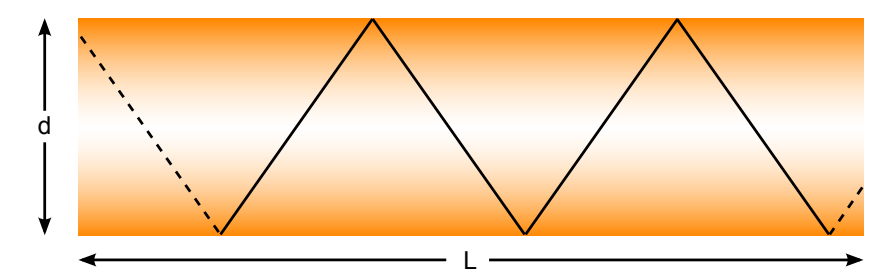

\begin{abstract}
Abbildung 5.10: Zick-Zack-Positionierung von sich nicht durchdringenden Stapelfehlern - Diese Skizze verdeutlicht die maximal erreichbare Defektdichte wenn sich die Stapelfehler nicht durchdringen
\end{abstract}

fehler spätestens dann durchdringen müssen, wenn sie nicht mehr in Zick-Zack-Weise durch den Draht positioniert werden können. Die Länge des gestörten Bereiches, der durch einen Stapelfehler erzeugt wird, beträgt mit den geometrischen Beziehungen aus Abbildung 5.10

$$
L_{S F 1}=\cot \alpha \cdot d
$$

Bei einer Gesamtlänge L des Drahtes ist somit die maximale Defektdichte für ungestörte Stapelfehler gegeben durch

$$
\rho=\frac{N_{\text {Defekte }}}{L}=\frac{L}{L_{S F}} \cdot \frac{1}{L}=\frac{1}{L_{S F}} \propto \frac{1}{d} .
$$

Wie allerdings im Abschnitt über den Verlauf der Defektdichte und in Abbildung 5.3 gezeigt wird, werden sich überschneidende Stapelfehlern entlang der beiden aktiven Gleitsysteme schon früh beobachtet. Demnach ist ein vorhandener Stapelfehler für die Bewegung einer Partialversetzung auf einem durchschneidenden Gleitsystem kein relevantes Hindernis. Dieses einfache Durchdringen der Stapelfehler wurde in vielen Zugversuchen auch mehrfach in einem Draht beobachtet. Dabei ist auszuschließen, dass die Stapelfehler auf unterschiedlichen Höhen durch den Draht laufen. Dies kann mit Aufnahmen wie in Abbildung 5.20 eindeutig gezeigt werden. In dieser Aufnahme wird deutlich sichtbar, dass die sich durchschneidenden Stapelfehler den gesamten Drahtquerschnitt einnehmen.

In seltenen Fällen konnte zudem ein weiteres Verhalten bei der Durchkreuzung zweier Stapelfehler beobachtet werden. In Abbildung 5.11 ist gezeigt, wie neben Stapelfehler 
und Zwillingslamellen, die komplett durch den Drahtquerschnitt laufen, auch andere Strukturen sichtbar sind. In drei Fällen treten Stapelfehler auf, die nicht durch den gesamten Draht laufen, sondern an einem Stapelfehler auf einem anderen Gleitsystem enden. Der Kontrast läuft auf keinem der beiden Gleitsysteme erkennbar weiter. Darüber hinaus ist auch kein anderer Dehnungskontrast an den Treffpunkten der Defekte zu erkennen. Dies deutet darauf hin, dass an den Schnittpunkten keine starke Gitterdehnung im Sinne einer vollen Versetzung existiert. Da die Konstellation dennoch besonders ist, wird in Kapitel 6 versucht, ein atomistisches Bild der sich ergebenden Struktur zu entwickeln.

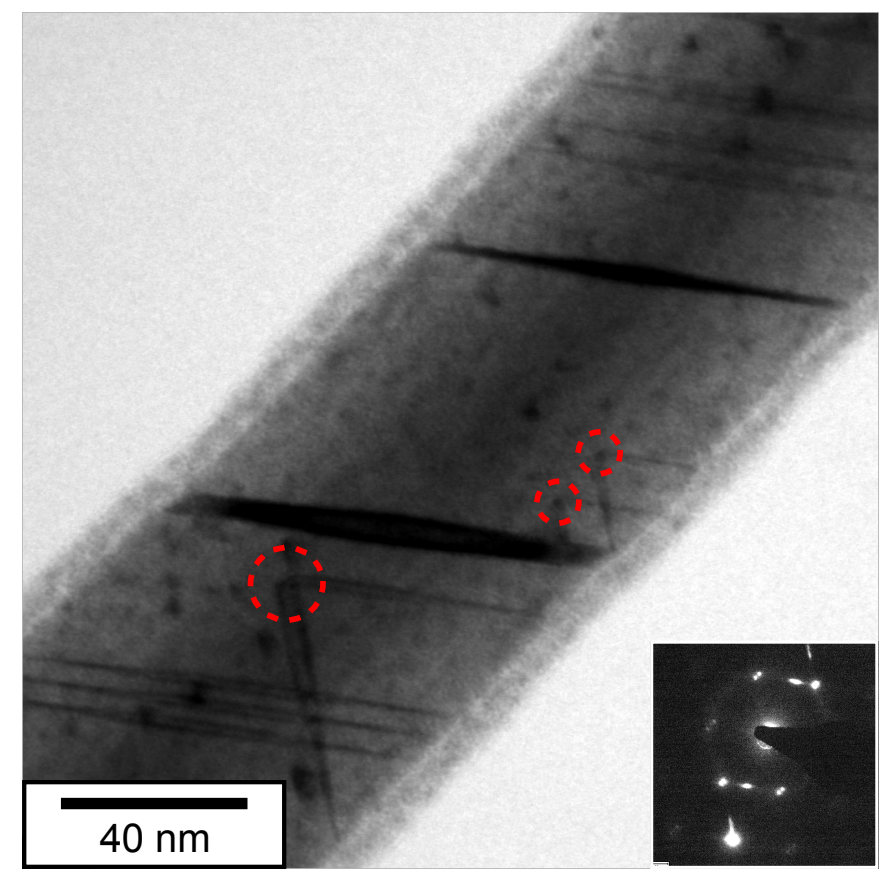

Abbildung 5.11: Nicht durchlaufende Stapelfehler - Hellfeldaufnahme eines verformten $60 \mathrm{~nm}$ Drahtes, die den seltenen Fall zeigt, wo Stapelfehler nicht durch den gesamten Draht laufen. Der Kontrast endet in diesem Fall an dem Punkt, wo sich zwei Stapelfehler auf sich durchschneidenen Gleitsystemen kreuzen.

\subsubsection{Analyse der Zwillinge}

Das Auftreten von Zwillingslamellen ist durch eine Analyse des Beugungsbildes während des Zugversuches nachweisbar. Durch die Spiegelung des Kristallgitters an einer $\{111\}$-Ebene können in der Elektronenbeugung für spezielle Zonenachsen neue Reflexe 


\section{ERGEBNISSE}

auftreten. Bei sieben Drähten, in denen Stapelfehler während der Verformung aufgetreten sind, konnte so eine Zwillingsbildung direkt nachgewiesen werden. Bei weiteren neun Drähten ergab die Analyse der gewählten Zonenachse während des Zugversuches keinen direkten Nachweis, allerdings lassen deutliche Kontrastunterschiede bei speziell gewählten Abbildebedingungen diesen Schluss zu (vgl. dazu auch Bild 5.12). Somit traten Zwillinge in Drähten dieser Kategorie mit einer Häufigkeit von 16 aus 22 Drähten in einem Größenbereich zwischen 45 und $280 \mathrm{~nm}$ auf. Bemerkenswert bei der Zwil-

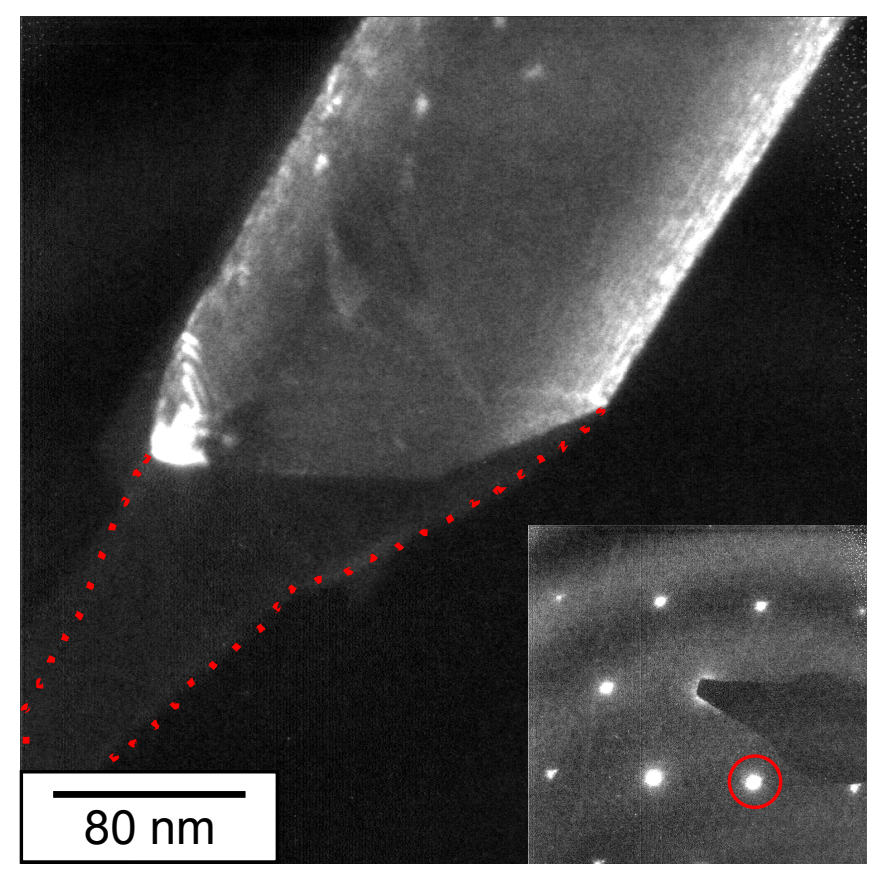

Abbildung 5.12: Zwillingsnachweis in einem Draht über den Kontrastwechsel - Diese $\{220\}$-Dunkelfeldaufnahme zeigt den Kontrastwechsel an der Spitze eines Drahtes mit $140 \mathrm{~nm}$ Durchmesser

lingsbildung ist darüber hinaus, dass in keinem Versuch eine Verzwilligung auf beiden aktiven $\{111\}$-Ebenen zu beobachten war. In den jeweiligen Beugungsbildern ist zum Teil deutlich erkennbar, dass auf zwei Gleitebenen zuerst Stapelfehler entstehen. Der Übergang vom "streaking" der zugehörigen Reflexe zum eindeutigen Zwillingsreflex ist jedoch immer nur in einer Richtung beobachtet worden.

Einhergehend mit der Spiegelung der Orientierung innerhalb des verzwillingten Bereiches ist auch eine Formänderung des Drahtes zwischen den Zwillingsgrenzen. In Ab- 


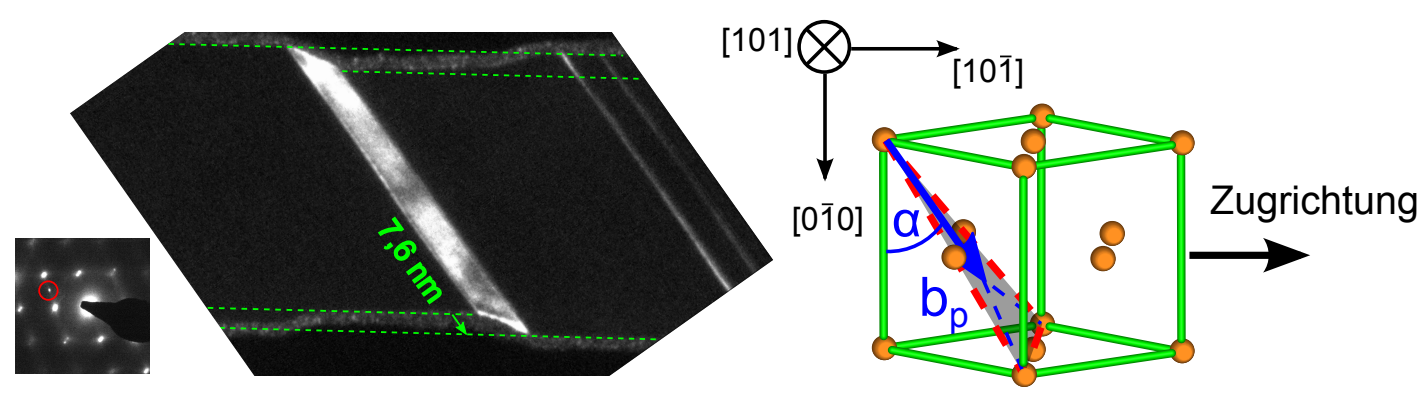

Abbildung 5.13: Durch Verformung entstandener Zwilling - Dunkelfeld Abbildung von einem 10,7 nm dicken Zwilling in einem verformten Draht mit 95 nm Durchmesser. Erkennbar ist die mit der Reorientierung verbundene Formänderung des Drahtes. Die Skizze rechts veranschaulicht die in dieser Abbildung vorliegende Geometrie.

bildung 5.13 ist ein Beispiel für einen solchen Fall gezeigt. Im Dunkelfeld ist erkennbar, dass die Zwillingslamelle eine Dicke von 10,7 nm aufweist. Zur besseren Sichtbarkeit wurde der zum Zwilling gehörende Reflex zur Bildgebung ausgewählt. Der Ebenenabstand in $\{111\}$-Richtung für Gold beträgt

$$
d_{(111)}=\frac{a_{\text {Gold }}}{\sqrt{1^{2}+1^{2}+1^{2}}}=2,35 \AA
$$

Nimmt man den Zwillingsbildungsmechanismus aus dem Kapitel 2.1 als Grundlage lässt sich dieser Zwilling als Resultat von benachbarter Partialversetzungsaktivität verstehen. Somit war die Bewegung von etwa 45 Partialversetzungen auf benachbarten \{111\}-Ebenen nötig, um diesen geschlossenen Zwilling zu bilden. Da jede Partialversetzung (mit dem höchsten Schmidfaktor von 0,471) die Oberfläche in der hier gewählten Projektion um

$$
d_{O F}=d_{[112]}=\frac{a_{\text {Gold }}}{\sqrt{1^{2}+1^{2}+2^{2}}}=1,66 \AA
$$

verschiebt, ist der Versatz zwischen ungestörter Oberfläche und Oberfläche am Ende der Zwillingslamelle

$$
\delta=45 \cdot 1,66 \AA=7,6 \mathrm{~nm}
$$

Auf der TEM Aufnahme wurde ebenfalls eine Verschiebung von 7,6 nm ausgemessen. Während der weiteren Verformung des Drahtes wurde beobachtet, dass diese Zwillingslamellen im Volumen wachsen können. Eine Sequenz eines solchen Wachstumsprozesses ist in der Bilderserie in Abbildung 5.14 dargestellt. 


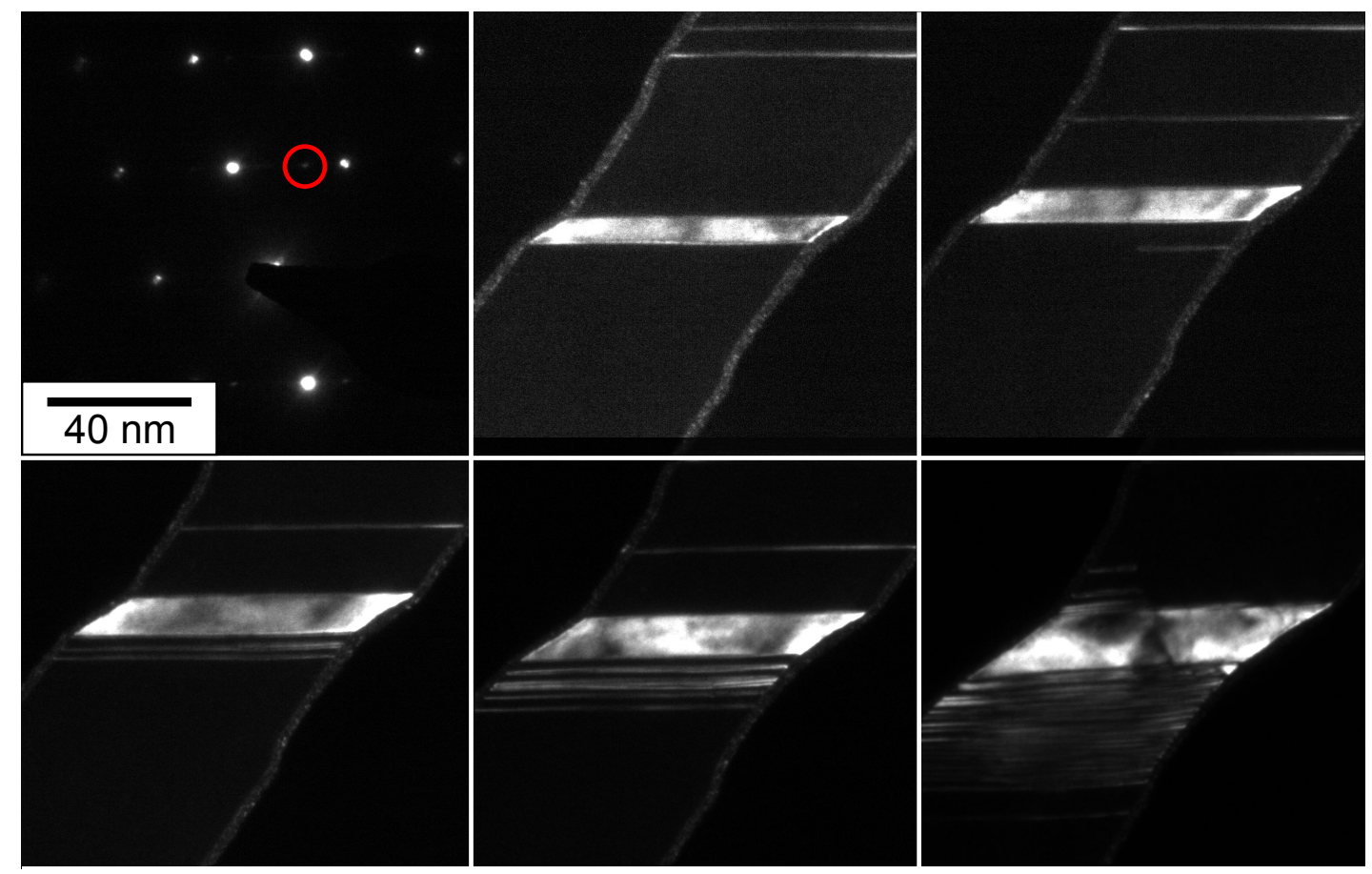

Abbildung 5.14: Zwillingswachstum - Sequenz von Dunkelfeldaufnahmen, die das Wachstum einer bestehenden Zwilingslamelle in einem $95 \mathrm{~nm}$ Draht zeigen. Der zur Bildgebung verwendete Reflex ist im ersten Teilbild markiert. 


\subsubsection{Dehnungsabschätzung}

Mit den vorangestellten Untersuchungen konnte eindeutig gezeigt werden, dass im Laufe der Verformung Stapelfehler und Zwillinge im Draht gespeichert werden. Daraus wurde abgeleitet, dass die Verformung im Wesentlichen durch die Aktivierung und Bewegung von Partialversetzungen auf zwei $\{111\}$-Ebenen getragen wird. Allerdings bleibt dabei offen, ob dies die einzigen beteiligten Defekte sind. Bei den untersuchten Probengrößen zwischen 40 und $280 \mathrm{~nm}$ ist die Aufenthaltsdauer einer sich frei bewegenden Versetzung sehr kurz (detaillierte Rechnungen dazu wurden in Kapitel 4 durchgeführt). Damit ist auch nicht ausgeschlossen, dass eine Verformung zum Beispiel durch die Aktivierung von vollen Versetzungen stattfindet, die keine sichtbare Spur im Draht hinterlässt. Diese Fragestellung konnte bei einem durchgeführten Zugversuch an einem $60 \mathrm{~nm}$ Draht angegangen werden. In diesem Versuch waren im Laufe eines Videoteils zwei Defekte die gesamte Zeit über sichtbar und konnten somit als fixe Marker benutzt werden. Die Position dieser Marker ist in Abbildung 5.15 durch die gestrichelten Linien gekennzeichnet. Nun wurde zwischen jeweils zwei aufeinander folgenden Einzelbildern das Auftreten eines neuen Kontrastes zwischen den Markerdefekten gezählt. In diese Zählung ein-

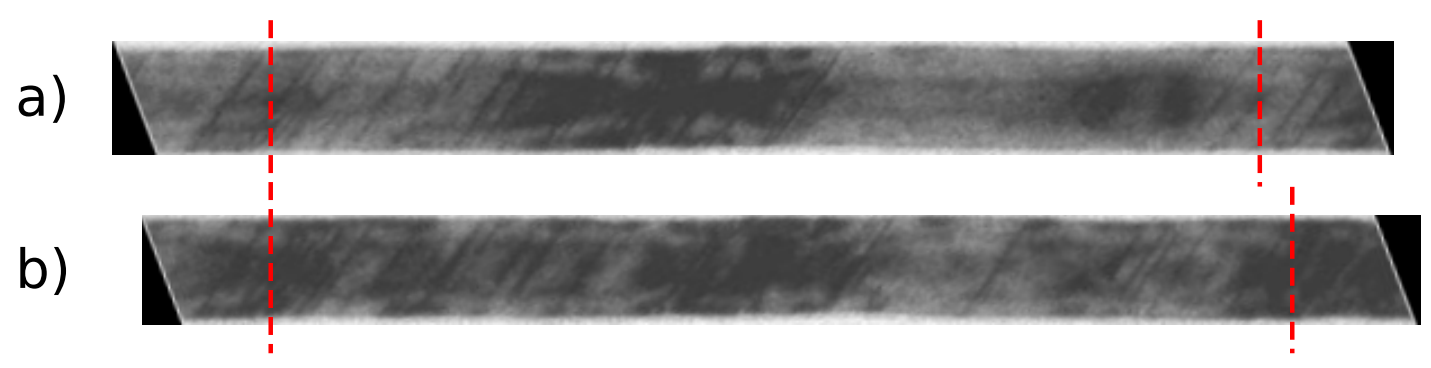

Abbildung 5.15: Ermittlung der Dehnung zwischen Markerdefekten in einem 60 nm Draht - a) - Vorgedehnter Draht mit Marker-Defekten b) - Dehnung des Drahtes mit Verschiebung eines der Marker

geschlossen wurden zudem nicht nur neu entstehende Defekte, sondern auch sichtbare Kontraständerungen an bestehenden Defekten und im Drahtvolumen. Dadurch sollte sichergestellt werden, dass aufgrund der Abbildebedingung im TEM möglichst die Gesamtheit der Defekte erfasst wird.

Für die Auswertung der zugehörigen Dehnung wurde die Annahme getroffen, dass es sich bei den kontrastverändernden Defekten um die Partialversetzungen handelt, die den höchsten Schmidfaktor nach Tabelle 2.1 haben. Die gewählte Zonenachse für die 


\section{ERGEBNISSE}

Abbildung ist dabei identisch zu der in Abbildung 5.13, sodass sich jedem Defekt relativ einfach eine Längenänderung zuordnen lässt. Durch die Orientierung des Drahtes in [110]-Zonenachse sind die geometrischen Beziehungen dieselben wie sie in Abbildung 5.13 dargestellt werden.

Man kann nun die Anzahl der gezählten Defekte und damit die rechnerisch ermittelte Dehnung mit der gemessenen Dehnung zwischen den Markern vergleichen. Die Längenänderung, die eine Partialversetzung auf einer $\{111\}$-Ebene bei Dehnung in $<110>-$ Richtung verursacht, beträgt

$$
\delta l_{P V}=\left|\overrightarrow{b_{p}}\right| \cdot \cos (\alpha)=0,096 \mathrm{~nm} .
$$

Hierbei bezeichnet $\alpha$ den Winkel zwischen $\{111\}$-Ebene und Zugachse, wie es in Abbildung 5.13 eingezeichnet ist. In der ausgewählten Zeitsequenz wurden zwischen den beiden Markerpositionen 71 Defekte gezählt. Die damit verbundene Verlängerung des Drahtes beträgt mit obiger Annahme

$$
\delta l_{\text {Draht }}=71 \cdot \delta l_{P V}=6,8 \mathrm{~nm} .
$$

Die gemessene Verlängerung aus den Einzelbildern des Videos beträgt $13 \mathrm{~nm}$ mit einer Ungenauigkeit von etwa $1 \mathrm{~nm}$. Damit ergibt sich eine Diskrepanz in der ermittelten Dehnung von etwa $6 \mathrm{~nm}$.

Ausgehend von diesem Ergebnis sind mehrere Erklärungen für die Abweichungen möglich. Denkbar ist zum einen, dass ein detektierter Kontrast nicht ein intrinsischer Stapelfehler ist, sondern durch die Bewegung von mehreren Partialversetzungen auf benachbarten Ebenen sich ein extrinsischer Stapelfehler oder ein sehr dünner Zwilling gebildet hat. Dies führt dazu, dass eine zu geringe Längenänderung des Drahtes angenommen wird. Da die für die Auswertung verwendeten Einzelbilder des Videos eine Auflösung von etwa $1 \mathrm{~nm}$ pro Pixel haben, der Ebenenabstand in (111)-Richtung aber nur 2,35 $\AA$ beträgt, ist mit einer einfachen Abschätzung vorstellbar, dass an einen detektierten Kontrast im Video bis zu 4 Partialversetzungen auf benachbarten Ebenen beteiligt waren. Die Anzahl der somit möglichen nicht erkannten Defekte könnte die Diskrepanz alleine abdecken. Rechnerisch müssten insgesamt

$$
13 \mathrm{~nm} / \delta l_{P V}=136
$$


Partialversetzungen aktiv gewesen sein, um die ermittelte Dehnung zu erreichen. Dies ist etwa ein Faktor zwei über der Anzahl an gezählten Kontrasten. Vergleicht man dies mit der maximalen Anzahl von 4 benachbarten $\{111\}$-Ebenen, die einen Kontrast bilden können, erscheint die Anzahl als realistisch darstellbar.

Zum anderen kann man aber auch die Annahme der obigen Überlegungen, dass ausschließlich Partialversetzungen die Verformung tragen, hinterfragen. Denkbar ist auch, dass volle Versetzungen zur Verformung beigetragen haben. Diese würden in der Untersuchung des Videos nicht erkannt werden, da außer einer Oberflächenstufe keine weitere Spur dieser Aktivität zurückbleibt. Trotzdem würde diese Bewegung zu einer Verlängerung zwischen den Markern führen.

Fasst man die beiden Punkte zusammen kann man folgern, dass unter Berücksichtigung der oben genannten Effekte und der technischen Randbedingungen die Anzahl der in den Einzelbildern gezählten Defekte nur eine untere Grenze darstellen kann. Dies bedeutet wiederum für die so ermittelten Dehnungswerte, dass diese ebenfalls die untere Grenze darstellen und somit durchaus eine Diskrepanz zu der gemessenen Dehnung zu erwarten ist. Andererseits ist mit diesem Untersuchungsergebnis auch nicht auszuschließen, dass während der Verformung volle Versetzungen zur Längenänderung beigetragen haben.

\subsubsection{Bruchverhalten}

Der überwiegende Teil (16 von 22) der gezogenen Drähte, die als charakteristischen Defekt einen Stapelfehler zeigen, weist einen Zwilling an der Stelle auf, wo der Bruch stattfindet. Dabei befinden sich oftmals verzwillingte Bereiche an beiden Enden des Drahtes. Der finale Bruch findet folglich im Volumen des invertierten Kristalls statt und nicht an der Grenze zum Material mit der ursprünglichen Orientierung. Eine beispielhafte Bruchkante ist in Abbildung 5.16 gezeigt.

Innerhalb eines schmalen verzwillingten Bereiches ändert sich durch die gespiegelte Symmetrie die kristallografische Richtung, die entlang der Zugachse liegt. Im Ausgangszustand liegt die Zugachse entlang der $<110>$-Richtung. Durch die Spiegelung des Kristalls an der begrenzenden $\{111\}$-Ebene verändert sich die Zugachse im Zwilling hingegen und liegt entlang der $<114>$-Richtung. Diese Orientierungsänderung ist in Abbildung 5.17 schematisch dargestellt. Mit der Spiegelung im Zwilling ändern sich 


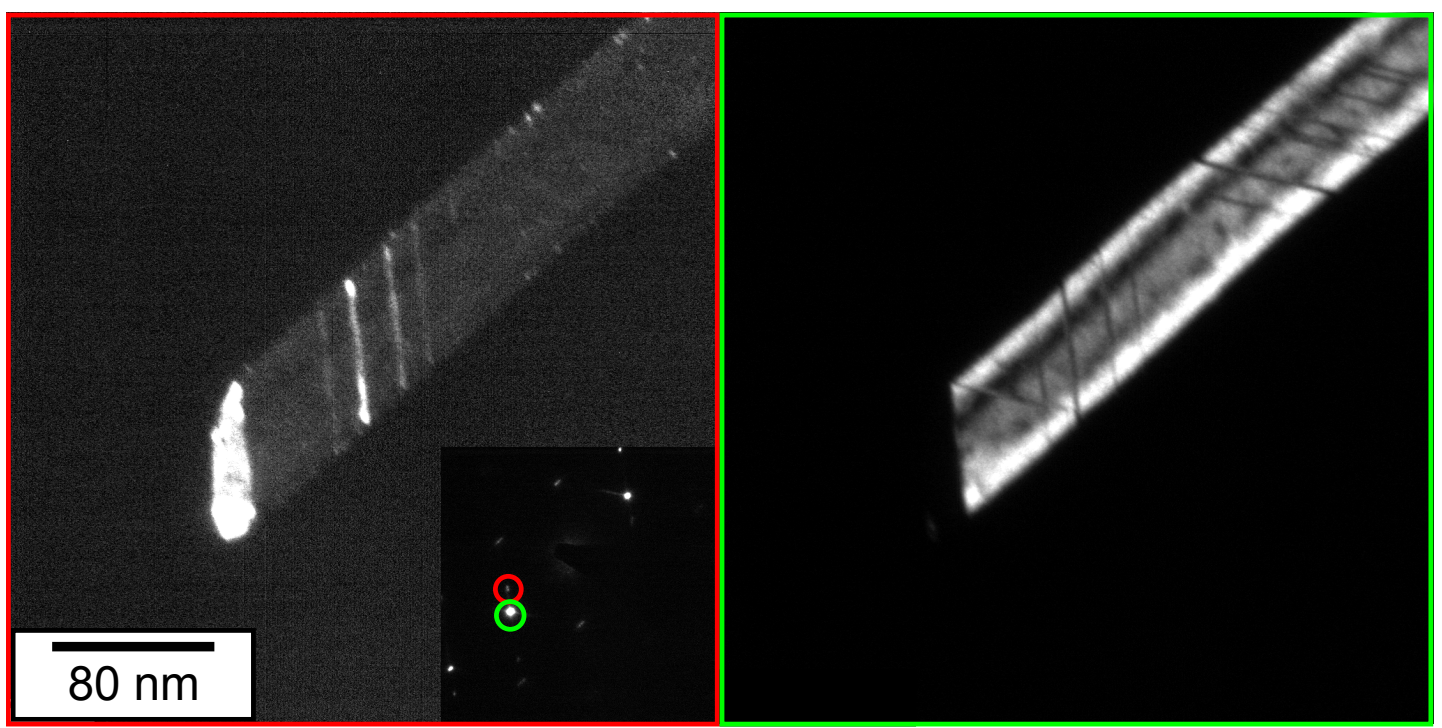

Abbildung 5.16: Bruchverhalten eines Drahtes mit Zwillingen - Die beiden Dunkelfeldaufnahmen zeigen das typische Bruchverhalten von Drähten, die während der Verformung Zwillinge ausbilden. In diesem $60 \mathrm{~nm}$ Draht erfolgte der Bruch innerhalb eines verzwillingten Bereiches. Farblich markiert ist dabei, welcher Reflex zur Bildgebung verwendet wurde.

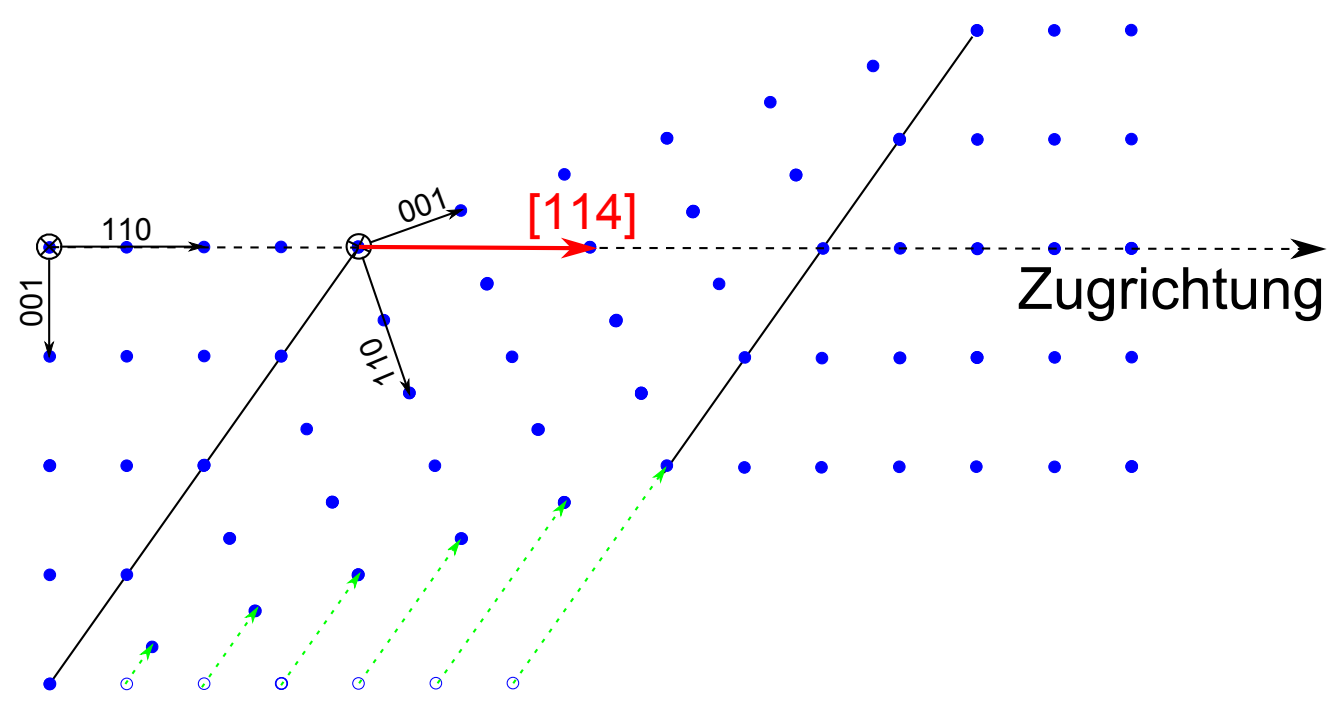

Abbildung 5.17: Zwillingsgeometrie - Schematische Darstellung der kristallographischen Reorientierung der Zugachse innerhalb einer Zwillingslamelle. Im Ausgangszustand liegt diese entlang der $<110>$-Richtung. Im Zwilling liegt sie entlang einer $<114>$-Richtung 


\begin{tabular}{ccccc} 
Gleitebene & $\begin{array}{c}\text { Volle } \\
\text { Versetzung }\end{array}$ & Schmidfaktor & $\begin{array}{c}\text { Partial- } \\
\text { versetzung }\end{array}$ & Schmidfaktor \\
\hline \hline$(111)$ & {$[1 \overline{1} 0]$} & 0 & {$[\overline{1} \overline{1} 2]$} & 0,471 \\
& {$[\overline{1} 01]$} & 0,408 & {$[1 \overline{2} 1]$} & 0,236 \\
& {$[0 \overline{1} 1]$} & 0,408 & {$[\overline{2} 11]$} & 0,236 \\
\hline$(\overline{1} \overline{1} 1)$ & {$[1 \overline{1} 0]$} & 0 & {$[112]$} & 0,262 \\
& {$[101]$} & 0,227 & {$[\overline{1} 21]$} & 0,131 \\
& {$[011]$} & 0,227 & {$[2 \overline{1} 1]$} & 0,131 \\
\hline$(\overline{1} 11)$ & {$[110]$} & 0,181 & {$[1 \overline{1} 2]$} & 0,419 \\
& {$[101]$} & 0,454 & {$[\overline{1} \overline{2} 1]$} & 0,052 \\
& {$[0 \overline{1} 1]$} & 0,272 & {$[211]$} & 0,367 \\
\hline$(1 \overline{1} 1)$ & {$[110]$} & 0,181 & {$[\overline{1} 12]$} & 0,419 \\
& {$[\overline{1} 01]$} & 0,272 & {$[121]$} & 0,314 \\
& {$[011]$} & 0,454 & {$[\overline{2} \overline{1} 1]$} & 0,052 \\
\hline \hline
\end{tabular}

Tabelle 5.1: Schmidfaktoren möglicher Gleitsysteme für einen Zugversuch in [114]Richtung

auch die rechnerisch aktiven Gleitsysteme. War es im Ausgangsfall noch ein symmetrischer Zugversuch mit mehreren gleichberechtigten Gleitsystemen (vgl. Tabelle 2.1), so liegt im Zwilling eine komplexere Situation vor. Für die veränderte Zugachse innerhalb des Zwillings sind die Schmidfaktoren in Tabelle 5.1 zusammengefasst. Dieser Aufstellung ist zu entnehmen, dass es für volle Versetzungen zwei gleichberechtigte Gleitsysteme mit einem Schmidfaktor von 0,454 gibt. Diese liegen von der aufgelösten Scherspannung noch über dem vergleichbaren Gleitsystem im Ausgangsmaterial. Für Partialversetzungen ist der höchste errechnete Schmidfaktor 0,471. Die Verschiebungsrichtung, die mit dieser Partialversetzung allerdings verknüpft ist, ist dieselbe, wie schon bei der Partialversetzung, die den Zwilling erzeugt hat. Der Grund dafür ist, dass die $\{111\}$-Ebene, auf der die Abgleitung stattfindet, unter der Zwillingsbildung unverändert erhalten bleibt. Die entstehende Konfiguration würde dann aus zwei sich benachbarten \{111\}-Ebenen mit gleicher Atomposition bestehen (vgl. dazu auch Abschnitt 2.1.1). Für die Bildung eines Stapelfehler dieser Art wäre eine sehr große Energie vonnöten. Ein solcher Fehler wurde experimentell nie beobachtet und daher ist auch keine weitere Verformung entlang dieser [112]-Richtung zu erwarten. Die als nächste bevorzugte Gleitsysteme sind volle Versetzung auf der (1111)-und (11̄1)-Ebene. 


\section{ERGEBNISSE}

Diese weisen einen Schmidfaktor von 0,454 auf.

Alle oben verwendeten Schmidfaktoren gelten allerdings nur, wenn durch die sehr dünne Zwillingslamelle eine reine Parallelverschiebung der Drahtenden bewirkt wird. Dies ist aber nur dann der Fall, wenn die Dicke der Lamelle sehr klein gegenüber der Drahtlänge ist. Für dickere Lamellen ist diese Näherung nicht mehr gültig, da es aufgrund der fixen Endpunkte des Drahtes auch zu einer Rotation kommen muss, die die Translation ausgleicht. Damit ist die Richtung der Zugachse nicht mehr durch eine einfache Beziehung aus der Spiegelsymmetrie ableitbar.

Bei den in diesem Kapitel betrachteten Drähten betrug die Gesamtdrahtlänge etwa $5 \mu \mathrm{m}$ und die Zwillingslamelle war nie breiter als $50 \mathrm{~nm}$. Die seitliche Translation, die mit einer solchen verzwillingten Lamelle verknüpft ist, beträgt demnach maximal $\delta x=35 \mathrm{~nm}$. Mit dieser Verschiebung ist eine Rotation um den Winkel

$$
\alpha=\arctan \left(\frac{\delta x}{L_{\text {Draht }}}\right)=0,40^{\circ}
$$

verknüpft (vgl. dazu Rechnung in Kapitel 5.1.3). Aus der obigen Berechnung folgt direkt, dass man maximal $0,4^{\circ}$ von der kristallographisch hergeleiteten Zugachse entlang der $<114>$-Richtung im Zwilling entfernt ist.

Diese kleine Rotation hat wiederum zur Folge, dass sich die Schmidfaktoren geringfügig ändern. Im Falle der Partialversetzung mit dem Schmidfaktor von 0,471 lässt sich die Auswirkung durch die Winkeländerung berechnen. Es ergibt sich eine Erhöhung des Schmidfaktors auf 0,474. Diese Partialversetzung ist jedoch nicht von Relevanz für die weitergehende Verformung wegen der vorher erwähnten resultierenden Koordination. Die anderen beteiligten Gleitebenen liegen allerdings nicht in der Zeichenebene und damit ist die Auswirkung der Rotation für diese Fälle nicht mehr einfach analytisch zu fassen. Die Größenordnung der zu erwartenden Änderung in den Schmidfaktoren ist aber durch die vorher angeführte Berechnung abzuschätzen. Demnach muss man durch die Rotation des Kristalls die Schmidfaktoren nur in der dritten Nachkommastelle anpassen. Die Reihenfolge der aktiven Gleitsysteme ändert sich dadurch jedoch nicht.

\subsubsection{Länge des Verformungsbereiches}

Die Bildung von Stapelfehler am Anfang der Verformung ist - wie in Abschnitt 5.1.1 betrachtet - von räumlich verteilten Nukleationsprozessen bestimmt. Daraus ergibt sich, 
dass der Bereich der Verformung nicht stark lokal eingeschränkt ist, sondern weitestgehend homogen über die Drahtlänge verteilt ist. An den Randbereichen des eingespannten Drahtes nimmt die Defektdichte allerdings meist ab. Ein Beispiel ist in Abbildung 5.18 dargestellt. Ein Grund für die Abnahme der Defektdichte zu den Rändern hin

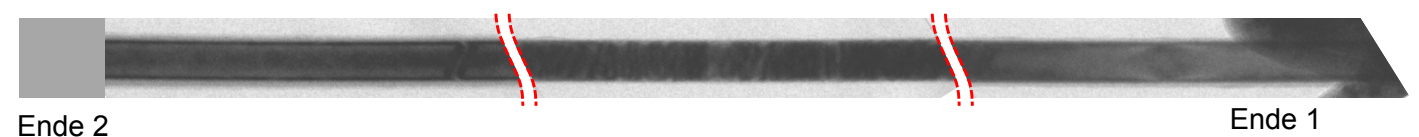

Abbildung 5.18: Übersicht über den Verformungsbereich - Zusammengesetzte Hellfeldaufnahme während der Verformung eines $50 \mathrm{~nm}$ Drahtes. Nahe den Enden sind sehr wenige Defekte zu erkennen. Im übrigen Teil symmetrisch um die Mitte des Drahtes ist die Defektdichte deutlich höher.

kann die Platinschicht auf dem Draht sein. Wie in Kapitel 4.2 erläutert wurde für die Befestigung der Drähte auf der Rahmenstruktur so genanntes Elektronenstrahl-Platin verwendet. Dies legt sich als dünne Schicht auf den gesamten Draht. Weit weg von den Klebestellen beträgt die Schichtdicke in Abbildung 5.18 etwa 2-3 nm. Nahe an den Rändern und damit an den Befestigungspunkten beträgt die Platindicke 10-20 nm. Der Schematische Verlauf von der Befestigung bis zur Drahtmitte der Platinschicht ist in Abbildung 5.19 gezeigt. Nimmt man an, dass die Kraft, die am Befestigungspunkt auf

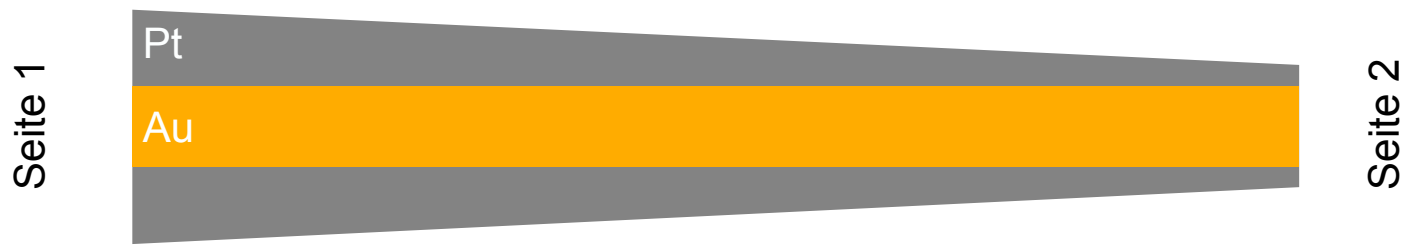

Abbildung 5.19: Schematische Darstellung der Platinschicht - Nahe der Befestigungspunkte ist die Platinschichtdicke größer als in der Mitte des Drahtes. Dadurch fällt die Spannung im Draht zu den Enden hin ab.

das System wirkt, gleich der Gegenkraft ist, die in der Drahtmitte wirkt, gilt

$$
\left|F_{\text {Seite1 } 1}\right|=\left|F_{\text {Seite } 2}\right|
$$

Für die Beschreibung der betrachteten Seiten wird im Folgenden nur noch die Zahl verwendet. 


\section{ERGEBNISSE}

Drückt man nun die Kraft aus über die Spannung auf die jeweiligen Flächenanteile $\mathrm{A}_{i}$ ergibt sich

$$
\sigma_{A u 1} \cdot A_{A u 1}+\sigma_{P t 1} \cdot A_{P t 1}=\sigma_{A u 2} \cdot A_{A u 2}+\sigma_{P t 2} \cdot A_{P t 2} .
$$

Während der Verformung wird nun ein Teil der Spannung vom Platin aufgenommen. Der Anteil kann errechnet werden, wenn für einen Punkt auf dem Draht in beiden Komponenten gleiche Dehnungen angenommen wird. Dies bedeutet

$$
\varepsilon=\frac{\sigma_{A u i}}{E_{A u}}=\frac{\sigma_{P t 1}}{E_{P t}}
$$

Zusätzlich werden der Übersichtlichkeit halber die beiden Elastizitätsmoduln durch deren Verhältnis ersetzt

$$
\tilde{E}=\frac{E_{A u}}{E_{P t}}
$$

und die konstante Fläche des Goldes $\mathrm{A}_{A u}$ auf beiden Seiten verwendet. Mit Hilfe dieser Beziehungen lassen sich nun die Spannungsanteile des Platins in Gleichung 5.13 ersetzen. Nach einer weiteren Umformung erhält man

$$
\sigma_{A u 1}=\sigma_{A u 2} \cdot\left(\frac{\tilde{E} A_{A u}+A_{P t 2}}{\tilde{E} A_{A u}+A_{P t 1}}\right) .
$$

Da nach Vorgabe in Abbildung 5.19 die Fläche des Platins auf der Seite 1 größer ist als auf der Seite 2 gilt

$$
\sigma_{A u 1}<\sigma_{A u 2}
$$

Mit dieser einfachen Betrachtung wird deutlich, dass durch eine dickere Platinschicht nahe der Befestigungspunkte eine geringere Spannung auf die Drähte wirkt. Der Einfluss auf den Spannungsverlauf wird deutlich, wenn man typische Werte für die beteiligten Materialien einsetzt. Für das verwendete Platin liegen keine Werte für die mechanischen Eigenschaften vor. Jedoch sind an vergleichbaren Systemem Untersuchungen durchgeführt worden, die einen Elastizitätsmodul zwischen 10 GPa bis $100 \mathrm{GPa}$ erwarten lassen [43]. Die Schichtdicke beträgt nahe an den Befestigungspunkten etwa $20 \mathrm{~nm}$ und in der Drahtmitte $5 \mathrm{~nm}$. Damit ergibt sich für einen Draht mit $100 \mathrm{~nm}$ langen Facetten nahe der Befestigung nur noch ein Spannungsniveau von

$$
\sigma_{A u 1}=73 \% \ldots 97 \% \quad \sigma_{A u 2}
$$

und somit zum Teil eine deutlich verringerte Spannung in Abhängigkeit davon, wie genau der Wert für den Elastizitätsmodul der Platinkomponente ist. Dies zeigt, dass 
das Vorhandensein der Platinschicht ein wichtiger Grund dafür sein kann, dass nahe an den Befestigungsstellen eine geringere Defektdichte beobachtet wurde und die Drähte hauptsächlich nahe der Mitte reißen.

\subsubsection{Einfluss des Elektronenstrahls}

Der Einfluss des Elektronenstrahls auf den beobachteten Verformungsmechanismus ist abhängig von der Energie und für einige Materialien nicht immer zu vernachlässigen. Wie im experimentellen Teil beschrieben, wurden die Zugversuche in einem TEM mit einer Beschleunigungsspannung von $300 \mathrm{kV}$ durchgeführt. Um abschätzen zu können, welcher Einfluss vom Elektronenstrahl zu erwarten ist, wurde die Schwellwertenergie $\mathrm{E}_{t}$ für Gold berechnet. Diese gibt an, ab welcher Energie (in $\mathrm{MeV}$ ) mit Elektronenstrahl induzierten knock-on Prozessen zu rechnen ist [28].

$$
E_{t}=\frac{\left(\frac{100+A E_{d}}{5}\right)^{1 / 2}-10}{20}
$$

Ausgehend von einem Atomgewicht bei Gold von A=197 a.u. und einer displacement energy $\mathrm{E}_{d}=36 \mathrm{eV}$ (aus [28]) ergibt sich eine Schwellwert Energie von $\mathrm{E}_{t}=1,40 \mathrm{MeV}$.

Wie bereits erwähnt lag die Energie der verwendeten Elektronen bei $300 \mathrm{keV}$, was somit deutlich unterhalb des ermittlelten Schwellwertes liegt. Auf Grundlage dieser Überlegung ist folglich mit keiner ballistischen Schädigung der Probe zu rechnen.

Schwieriger als den Schwellwert für knock-on Schädigung zu berechnen ist hingegen abzuschätzen, welchen Einfluss eine Probenerwärmung während der Einstrahlung mit Elektronen auf das Ergebnis eines Zugversuches hat. Generell wird für gut leitendende Materialien wie Metalle der Einfluss als relativ klein erachtet (vlg. dazu [28]). Dort wird angegeben, dass mit einer Probenerwärmung im Bereich von $1^{\circ} \mathrm{C}$ zu rechnen ist. Um dennoch diesen Effekt besser beurteilen zu können, wurden zwei verschiedene Kontrollexperimente durchgeführt. Zum einen wurde ein Experiment an einem $100 \mathrm{~nm}$ dicken Draht ohne Elektronenstrahl im TEM durchgeführt und zum anderen 3 Drähte untersucht, die bei lediglich $30 \mathrm{kV}$ Beschleunigungsspannung im REM getestet wurden (siehe auch Appendix A).

Im Falle des im TEM getesteten Drahtes kann keine Aussage über den zeitlichen Verlauf der Defektdichte gemacht werden. Dennoch zeigt die post mortem Analyse eine 


\section{ERGEBNISSE}

gleichmäßige Verteilung von planaren Defekten im Draht mit vergleichbarer Defektdichte wie sie zu Beginn dieses Abschnittes ermittelt wurde. Im Beugungsbild konnten sowohl Stapelfehler als auch Zwillinge identifiziert werden. Der Bruch des getesteten Drahtes geschah an einer Zwillingslamelle.

Die Auswertung der im REM gezogenen Drähte lieferte ein vergleichbares Bild. In allen Fällen waren planare Defekte in den getesten Drähten vorhanden. Auffällig war bei diesen Versuchen jedoch, dass die Defektdichte im Allgemeinen etwas niedriger war. Abbildung 5.20 zeigt zwei Dunkelfeldbilder von im REM getesteten Drähten. Im linken Teilbild sind einige sich durchdringende Stapelfehler zu erkennen. Das rechte Teilbild zeigt die Gesamtheit aller sichtbaren Defekte in einem $110 \mathrm{~nm}$ Draht.

Die Summe der Ergebnisse dieser Versuche zeigt, dass zwar kein Unterschied im beobachteten Defektyp, der Zwillingsbildung sowie des Bruchverhaltens vorliegt, aber eine andere Defektdichte ereicht wird. Eine mögliche Erklärung hierfür ist, dass im REM eine um den Faktor zwei höhere Dehnrate während der Verformung verwendet wird.

Mit dieser Art von Kontrollexperimenten sind zwei Dinge sichergestellt: Zum einen ist

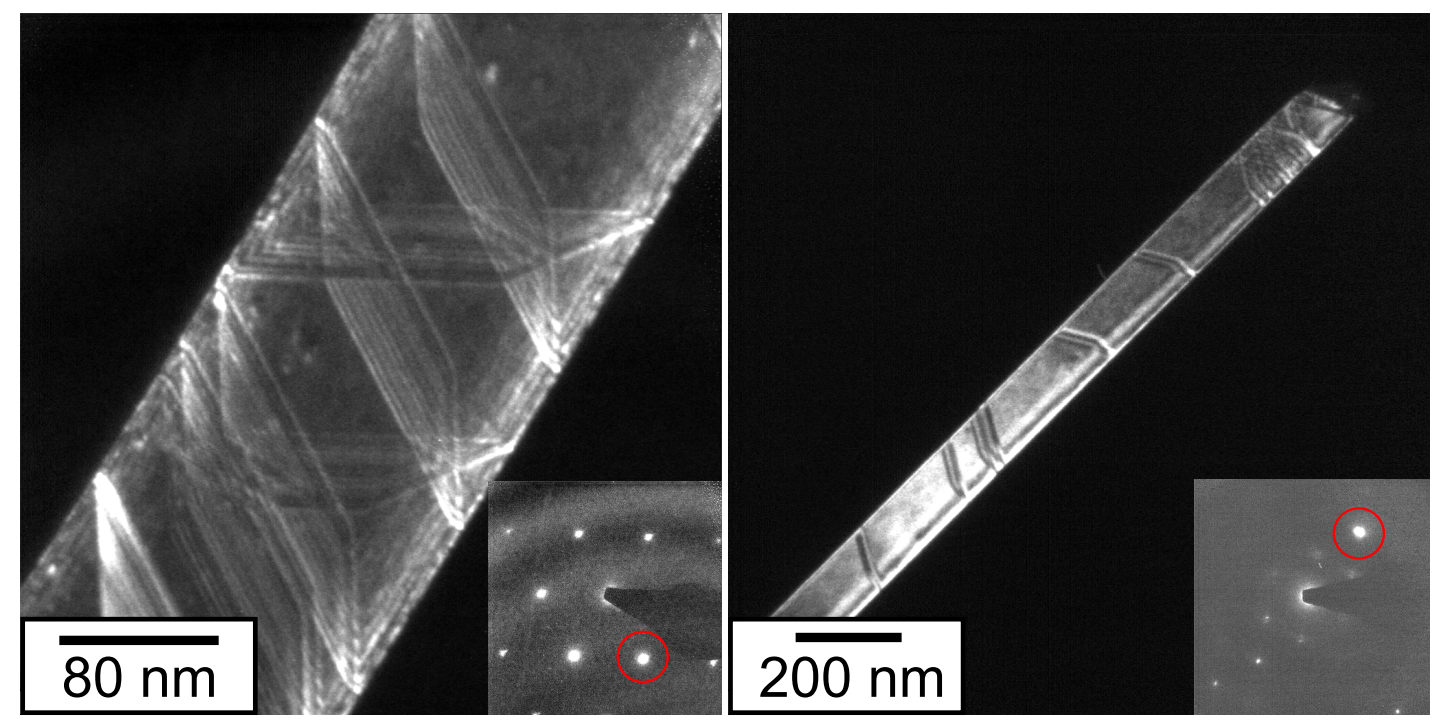

Abbildung 5.20: Einfluss des Elektronenstrahls - Dunkelfeldbilder von zwei im REM getesteten Dähten. Links ist eine weakbeam Aufnahme von Stapelfehlern in einem $160 \mathrm{~nm}$ Draht. Das rechte Dunkelfeldbild eines $110 \mathrm{~nm}$ Drahtes veranschaulicht, dass im Allgemeinen die Defektdichte in diesen Drähte geringer ist.

davon auszugehen, dass der verwendete Elektronenstrahl keinen Einfluss auf den beobachteten Defekttyp hat. Zum anderen ermöglicht die Vergleichbarkeit der Defekte eine 
direkte Interpretation der quantitativen Ergebnisse aus [24] für die Fließspannung. Die Werte für die Fließspannung finden im Kapitel 6 ihre Anwendung.

\subsubsection{Entlastungsversuch}

In einem speziellen Zugversuch wurde die Stabilität der Defekte bei einer Entlastung nach Beginn der plastischen Verformung untersucht. Der erste Teil des Tests verlief dabei analog zu den üblich getesteten Drähte. Der Draht wurde somit bis zum Einsetzen der Versetzungsnukleation und sichtbaren Stapelfehlern gezogen. Das Erscheinen von Stapelfehlern war dabei das Zeichen, dass die Spannung groß genug war, um den Draht plastisch zu verformen. Als nächster Schritt wurde an der Zughaltersteuerung nicht weiter verlängert, sondern der Entlastungsvorgang begonnen. Dabei wird die verfahrbare Seite auf die fixe Seite im Halter zu bewegt. Diese Umkehr des Belastungsvorganges macht sich sehr deutlich im TEM sichtbar, da die Position des Drahtes nun nachgeregelt werden musste. Die Defektdichte innerhalb des Drahtes hat sich während dieser Entlastung nahezu nicht verändert. Lediglich an 3 Positionen im Draht konnte eine Veränderung im Kontrast beobachtet werden. Diese sind in Abbildung 5.21 als vorhernacher Aufnahme gezeigt. Der Großteil des Drahtes behielt seine Kontraststruktur allerdings bei. Da die Defektdichte zu diesem Zeitpunkt schon sehr hoch war, konnten den Kontrasten keine individuellen Defekte zugeordnet werden. Eine genau Aussage über die Defekte an den veränderten Positionen lässt sich somit nicht treffen. Dennoch kann aufgrund der Vielzahl der Kontraste, die beim Belasten entstehen, geschlossen werden, dass das Reduzieren der Spannung kaum in eine Reduzierung der Defektdichte resultiert. 
a)

$80 \mathrm{~nm}$ b)

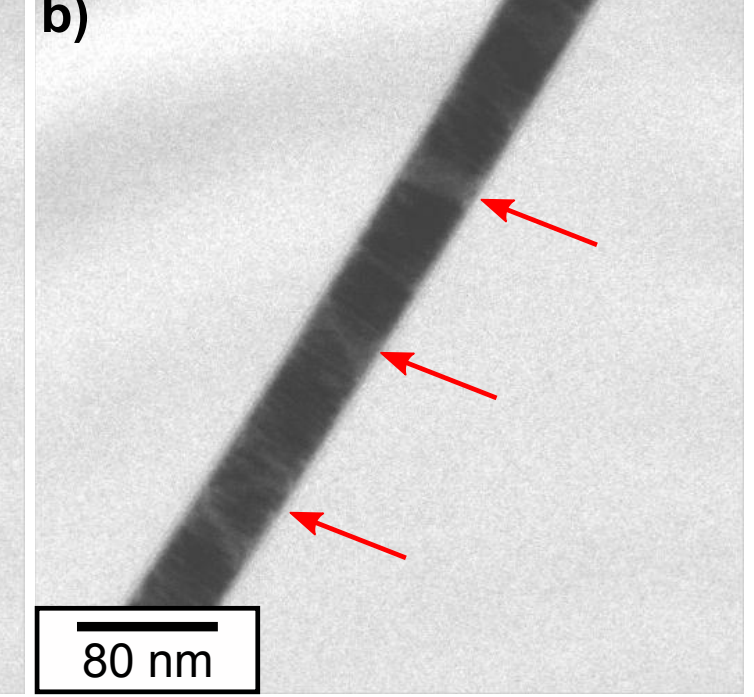

Abbildung 5.21: Hellfeldbilder des Entlastungsversuchs - a) Stapelfehler in einem $50 \mathrm{~nm}$ Draht beim Beginn der plastischen Verformung b) Defektdichte nach Entlastung durch Zusammenfahren der Fixierungspunkte im TEM-Halter 


\begin{tabular}{ccccc}
$\begin{array}{c}\text { Drahtbreite } \\
{[\mathrm{nm}]}\end{array}$ & $\begin{array}{c}\text { Länge } \\
{[\mathrm{nm}]}\end{array}$ & $\begin{array}{c}\text { Verformungs- } \\
\text { bereich [nm] }\end{array}$ & $\begin{array}{c}\text { Verhältnis der } \\
\text { Facettenlängen (ca.) }\end{array}$ & Überstruktur \\
\hline \hline 190 & 6000 & 700 & $4: 1$ & ja \\
200 & 5000 & 5000 & $5: 1$ & ja \\
330 & 5000 & 4000 & $5: 1$ & ja \\
240 & 5000 & 4000 & $4: 1$ & ja \\
300 & 5000 & 400 & $1: 1$ & nein \\
\hline \hline
\end{tabular}

Tabelle 5.2: Zugexperimente mit vollen Versetzungen - Übersicht über die verformten Proben, die volle Versetzungen zeigen. Angegeben ist neben der erscheinenden Breite im TEM auf die Länge, auch der die Drähte die Defekte zeigen im Verhältnis zu der getesteten Gesamtlänge.

\subsection{Zugversuche mit vollen Versetzungen}

Wie zu Beginn des Kapitels erwähnt konnten in den verformten Nanodrähten zwei verschiedene Defektypen beobachtet werden. Während der erste Teil sich dem Auftreten und der Analyse von Defekten gewidmet hat, die durch die Aktivierung von Partialversetzung entstanden sind, sollen in diesem Abschnitt nun die Ergebnisse für das Auftreten von vollen Versetzungen zusammengefasst werden.

Volle Versetzungen wurden während der Zugverformung in fünf Drähten beobachtet. Eine tabellarische Auflistung dieser Drähte ist in Tabelle 5.2 gegeben. Aus dieser Aufstellung sind einige Aspekte zu entnehmen. Zum einen fällt auf, dass die Breite, mit der die Drähte im TEM erscheinen, stets größer ist als $190 \mathrm{~nm}$. Zum anderen fällt auf, dass es Unterschiede in der Länge des Bereiches gibt, in dem die Drähte verformen. Während drei Drähte nahezu auf der gesamten Länge Defekte während der Verformung aufwiesen, zeigen zwei Drähte ein lokalisiertes Auftreten von Defekten. Jeweils ein Vertreter der beiden Verformungsverhalten ist in Abbildung 5.22 gezeigt. Im Falle der gleichmäßig verformenden Proben ist der zeitliche Verlauf zumindest qualitativ vergleichbar mit der Defektentwicklung aus 5.1. Mit der schrittweisen Erhöhung der Dehnung setzt in diesen Drähten die plastische Verformung ein und es sind Kontraste auf zwei $\{111\}$-Ebenen zu erkennen. Die Anzahl der sichtbaren Kontraste nimmt dabei stetig zu und führt zu einem stark inhomogenen Bild des Drahtes im Hellfeld (vgl. Teilbild a) in Abbildung 5.23). Die Art des Kontrastes der beobachteten Defekte unterscheidet sich dabei von dem der Defekte aus Abschnitt 5.1. Auffällig ist insbesondere, 


\section{ERGEBNISSE}

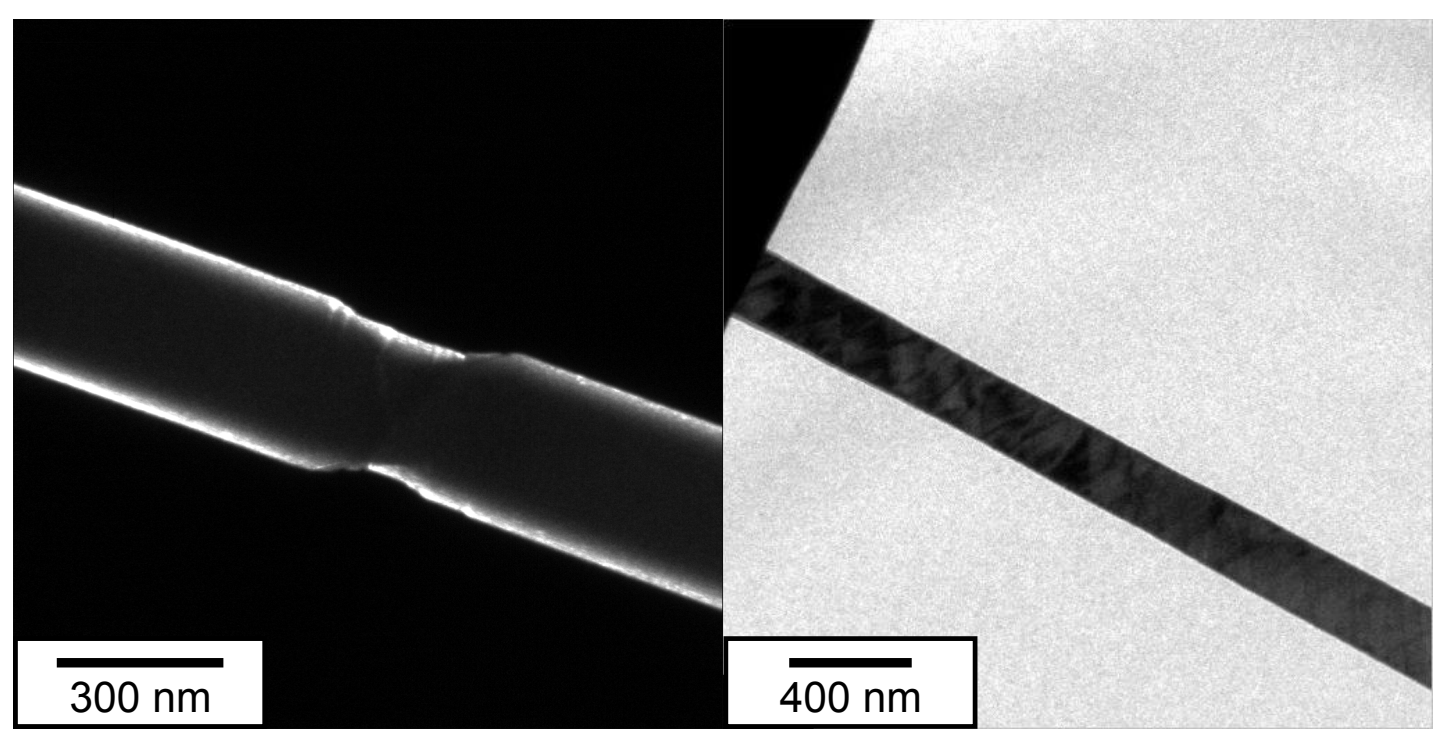

Abbildung 5.22: Vergleich der Verformungsbereiche - Darstellung der beiden unterschiedlichen Verformungsbereiche. Im linken Teilbild ist eine sehr lokalisierte Verformung in einem $300 \mathrm{~nm}$ breiten Draht zu sehen. im rechten Teilbild ist ein Ausschnitt eines 200 nm Drahtes gezeigt, der homogen über die Gesamtlänge verformt.

dass die Defekte nicht strikt entlang einer kristallographischen Ebene liegen, sondern auch gebogene Defektkontraste beobachtet werden können. Ein typisches Bild eines solchen Kontrastverlaufes ist in Abbildung 5.23 im Teilbild b) dargestellt. Auch fällt in dieser Abbildung auf, dass mit den Defekten kein oszillatorischer Kontrast verknüpft ist, wohl aber ein Dehnungsfeld existieren muss, dass sich im Hellfeldbild deutlich um die Defektlinien abzeichnet. Beide Effekte lassen den Schluss zu, dass sich volle Versetzungen in dem Draht befinden.

Neben diesen gebogenen starken Kontrasten finden sich auch sehr gerade schwache Kontraste entlang zweier Orientierungen. Diese sind im Dunkelfeldbild gut zu erkennen, wie in Abbildung 5.24 gezeigt. Die rote gestrichelten Linien markieren die beiden auftretenden Orientierungen. Diese geraden Kontraste können wahrscheinlich Oberflächenstufen zugeordnet werden, die durch Versetzungsbewegung auf $\{111\}$-Ebenen verursacht werden. Das schwache Dehnungsfeld an der Oberfläche kann im TEM als Kontrast wahrgenommen werden [44]. Bei der Mehrheit dieser detektierbaren Kontraste wird lediglich das erstmalige Auftreten im Draht beobachtet. Nur in seltenen Ausnahmefällen konnte eine kurzzeitig festsitzende Versetzung im Auftreten und Ver- 


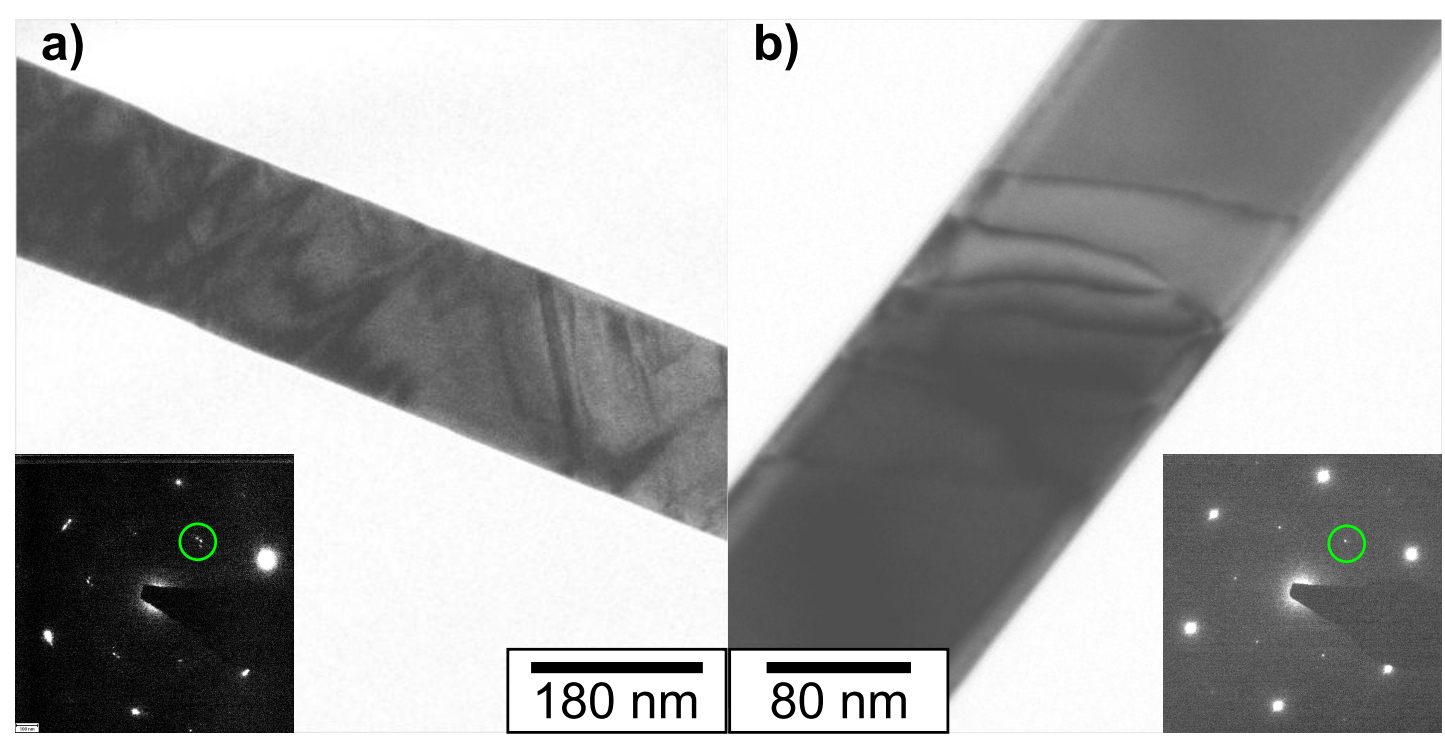

Abbildung 5.23: Defekte während des Zugversuches - Hellfeldaufnahmen während des Zugversuches zeigen a) Defekte auf zwei $\{111\}$-Ebenen in einem $200 \mathrm{~nm}$ Draht b) gebogene linienhafte Defekte in einem $190 \mathrm{~nm}$ Draht. In beiden (111)-Zonenachsen ist die Überstruktur erkennbar.

schwinden beobachtet werden. Ein solcher Fall, wo ein Versetzungssegment während des Versuches im Draht gespeichert wurde, ist in Abbildung 5.25 gezeigt. Der Kontrast lag über einige Verformungsschritte immobil im Draht. Mit fortschreitender Dehnung kann dann beobachtet werden, dass der Defekt verschwindet. Dies geschieht innerhalb eines Zeitintervalls von $50 \mathrm{~ms}$.

Das oben beschriebene Verformungsverhalten war in drei verschiedenen Drähten beobachtbar. In diesen Fällen konnte auch das Verhältnis von kurzer zu langer $\{111\}$-Facette durch Analyse der TEM Bilder zu jeweils über 4:1 bestimmt werden. Damit kann in diesen Fällen von plattenhaften Strukturen gesprochen werden. Auffällig ist weiterhin, dass in den beschrieben Drähten jeweils die Überstruktur in der $\{111\}$-Zonenachse beobachtet werden konnte.

In einem weiteren Draht, der keine Überstruktur zeigte, lag das Verhältnis der Facetten nahe bei eins. Dies konnte zwar nicht direkt gemessen werden, allerdings war die Transparenz des Drahtes im TEM sehr eingeschränkt. Dies lässt eine Abschätzung der unteren Grenze für die Dicke zu. Bei $300 \mathrm{kV}$ liegt diese nach Erfahrungswerten bei etwa $120 \mathrm{~nm}$. In diesem in jeder Dimension großen Draht war die Verformung lokalisiert auf einen Bereich, der zentral in der Mitte lag (siehe linkes Bild in Abbildung 5.22). Im üb- 


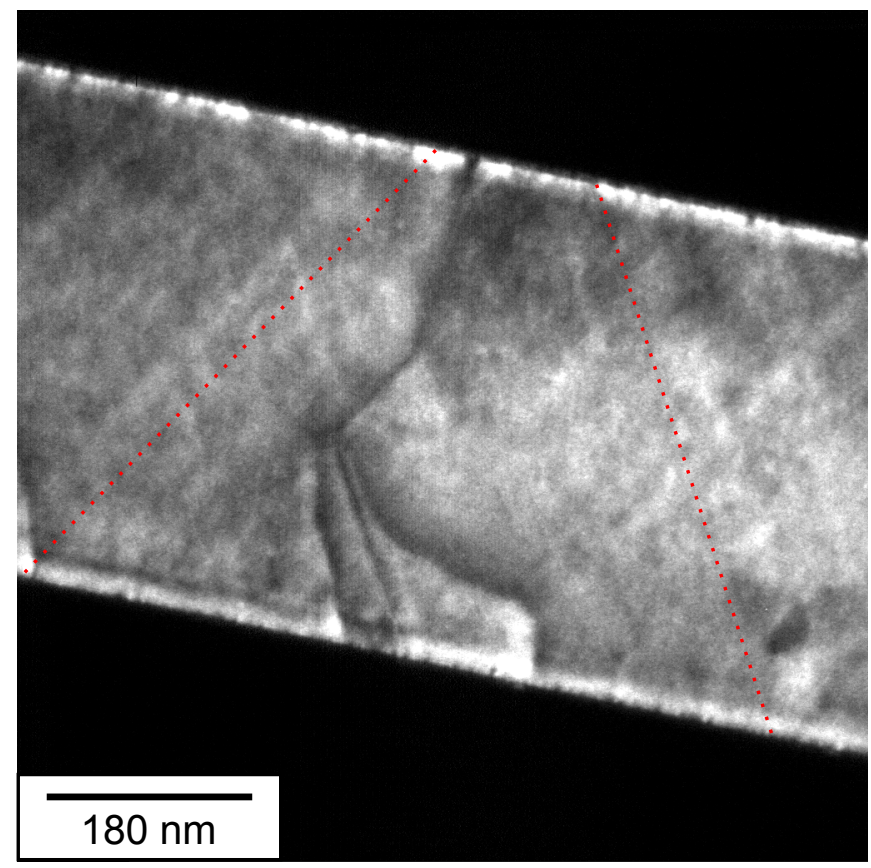

Abbildung 5.24: Oberflächenstufen im TEM - Dunkelfeldabbildung eines verformten $330 \mathrm{~nm}$ Drahtes. Deutlich erkennbar sind neben gebogenen Linienelementen auch schwache Kontraste durch Oberflächenstufen. Diese werden durch das Hinauslaufen der Versetzungen aus dem Draht erzeugt.

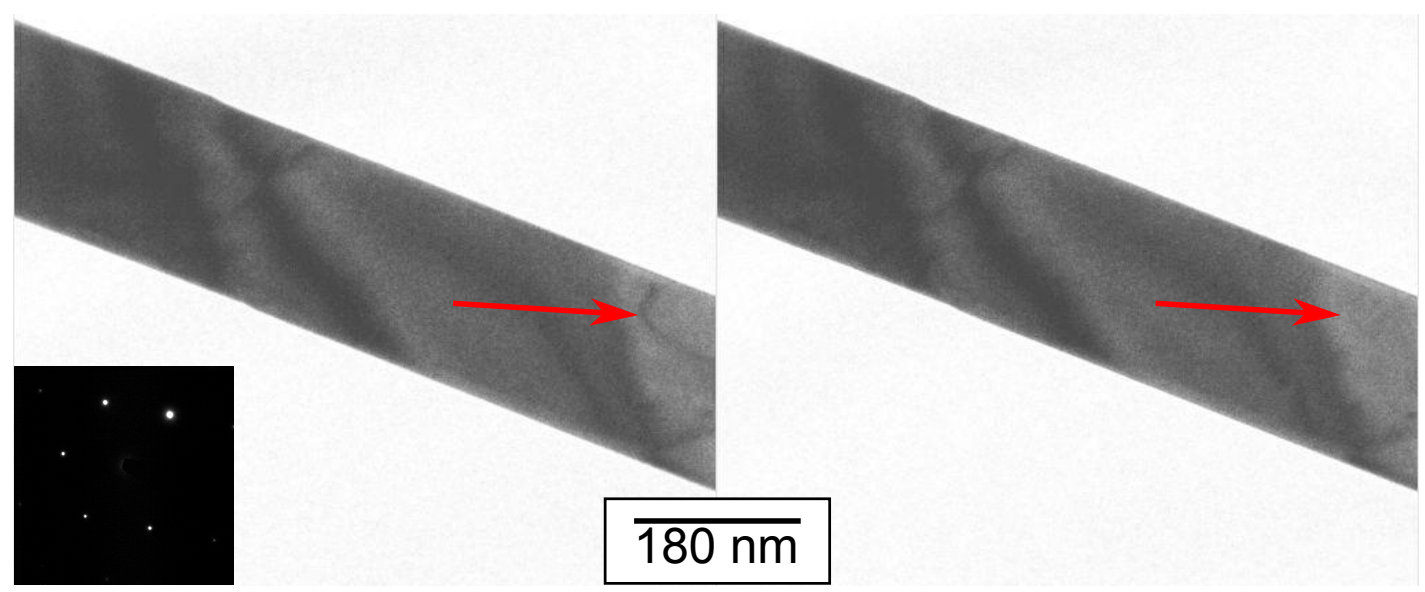

Abbildung 5.25: Hellfeldaufnahmen eines $190 \mathrm{~nm}$ Drahtes während des Zugversuches - Die Markierung zeigt ein gebogenes Versetzungssegment, das während der Verformung auftrat und zwischen zwei Einzelbildern verschwinde.t 
rigen Teil des Drahtes konnten keine Veränderungen der Oberfläche oder gespeicherte Defekte beobachtet werden. Der ersten Verjüngung folgte dann bei schrittweiser Erhöhung der Dehnung die Bildung einer dünnen filamentartigen Struktur zwischen den beiden Drahtenden, in der die Transparenz gegenüber dem unverformten Draht stark erhöht war. Dieser Zustand der Verformung ist in Abbildung 5.26 gezeigt. Die Akti-

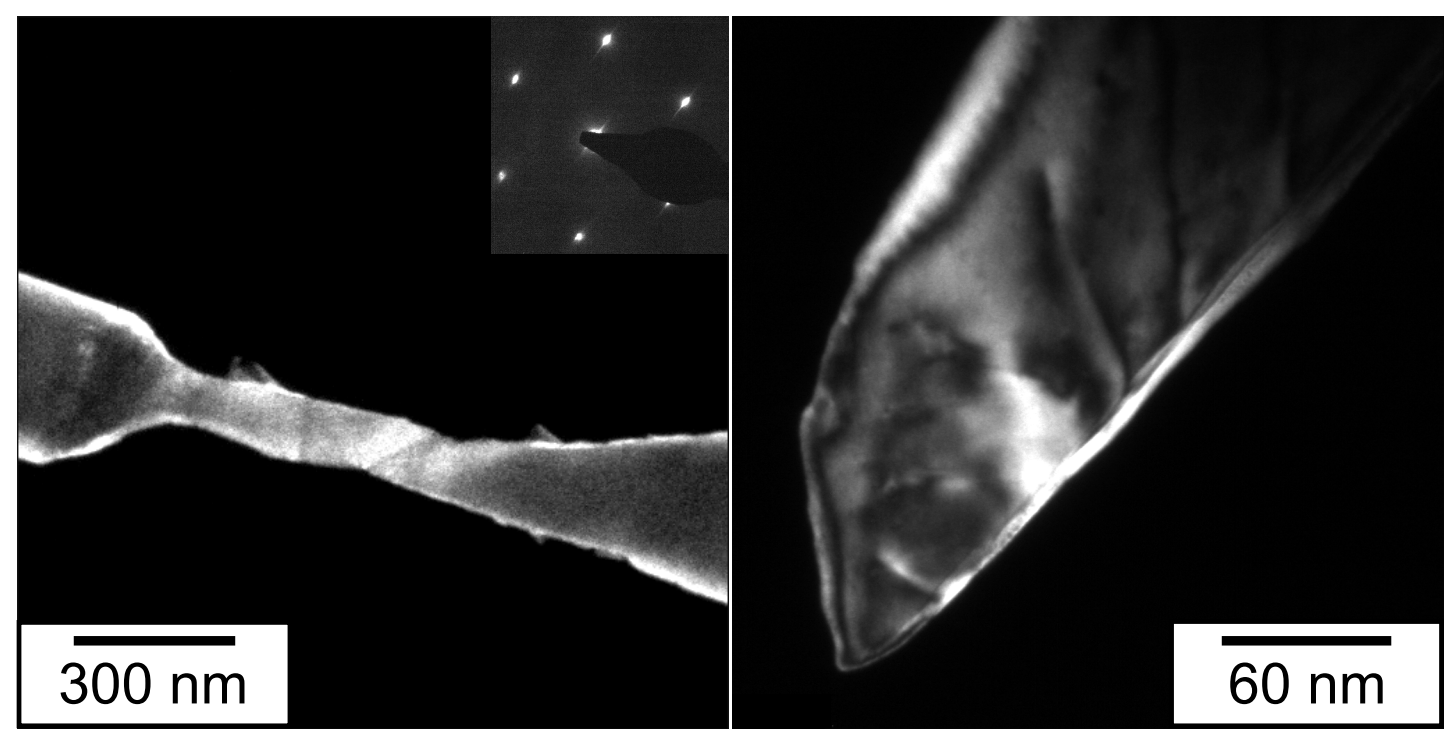

Abbildung 5.26: Verformung eines $300 \mathrm{~nm}$ Drahtes - Im linken Bild ist die sich ausbildende längliche Einschnürung im Dunkelfeldbild zu erkennen. In diesem dünnen Bereich sind nach dem Reissen Versetzungen eingelagert. Diese sind im Dunkelfeldbild rechts deutlich zu erkennen. Die (111)-Zonenachse zeigt keine Überstruktur.

vität von Versetzungen konnte erst nach dieser Einschnürung im Verformungsbereich erkannt werden. In diesem stark ausgedünnten Bereich um die Bruchstelle war dann eine genauere Analyse der Mikrostruktur möglich. Auch hier zeigten sich allerdings wie in den oben angeführten Beispielen als einzig erkennbare eingelagerte Defekte volle Versetzungen.

Ein weiterer Draht ist während der Verformung schlagartig gerissen. Hier konnte keine Defektanalyse während des Versuches vorgenommen werden. Nach dem Reißen zeigten sich in diesem $190 \mathrm{~nm}$ Draht, dessen Facetten ein Aspektverhältnis von etwa 4:1 aufwies, volle Versetzungen um die Bruchkante. Der übrige Teil des Drahtes zeigte dagegen keinerlei Defekte. Eine der Bruchkanten ist in Abbildung 5.27 im rechten Teilbild gezeigt. Auch dieser Draht wies wieder die zusätzlichen Reflexe in der $\{111\}$-Zonenachse auf. 


\section{ERGEBNISSE}

\subsubsection{Bruchverhalten}

Das Bruchverhalten und die räumliche Verteilung der Defekte innerhalb der Drähte liefert Hinweise auf die zugrunde liegenden Verformungsmechanismen. Wie schon bei der Länge des Verformungsbereiches lieferten die durchgeführten Zugversuche auch beim Bruchverhalten kein einheitliches Bild. Es konnten im Wesentlichen zwei unterschiedliche Bruchverhalten beobachtet werden. Zum einen zeigte der $300 \mathrm{~nm}$ Draht nach sehr lokal beginnender Verformung eine Einschnürung, innerhalb derer der Draht bis zum Bruch gedehnt wurde (vgl. 5.26). Alle anderen Drähte zeigten einen Scherbruch entlang einer etwa $60^{\circ}$ zur Zugachse orientierten Richtung. Zwei solcher typischen Bruchkanten mit den gespeicherten Versetzungen sind in Abbildung $5.27 \mathrm{zu}$ sehen.

Mit Hilfe einer Bildbearbeitungssoftware lassen sich die beiden Teile des gerissenen
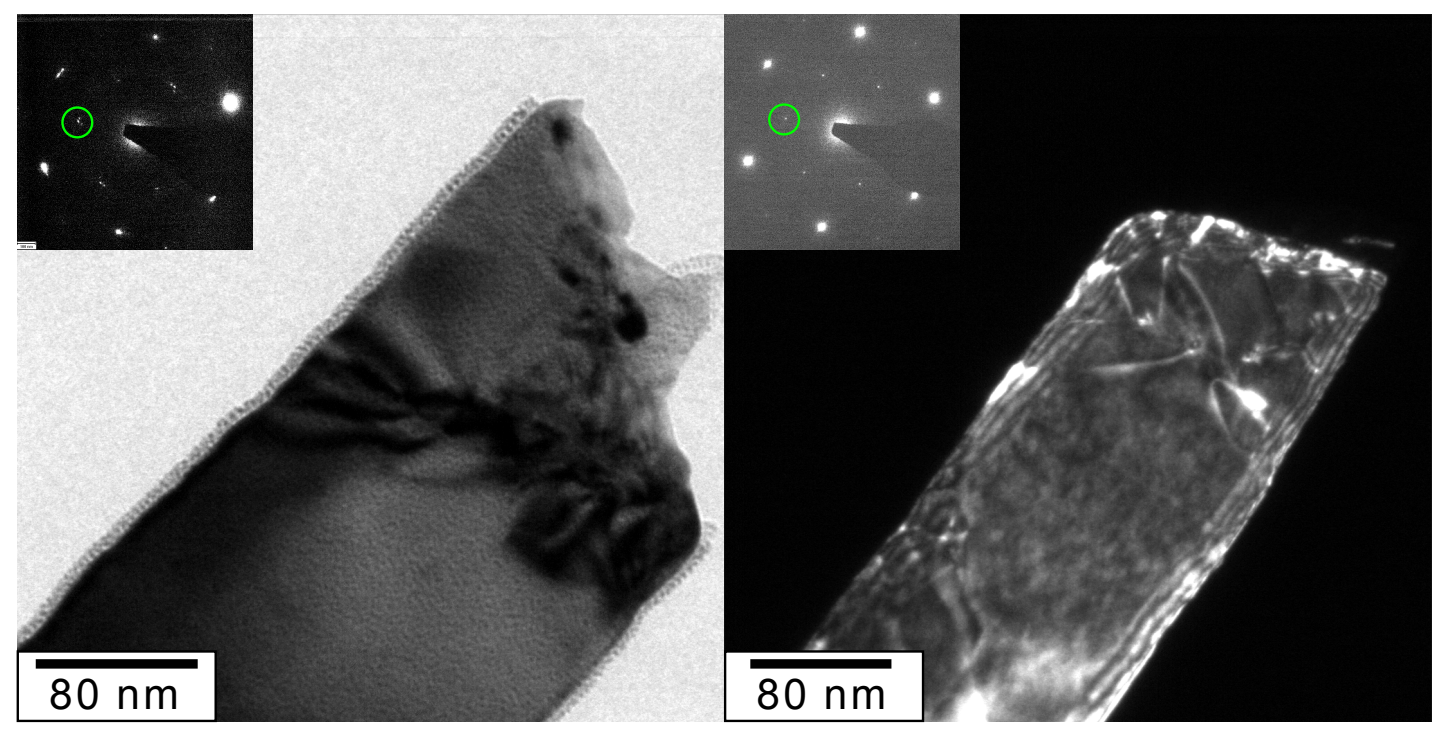

Abbildung 5.27: Bruchverhalten von Drähten mit vollen Versetzungen - Bruchkanten zweier Drähte (links 240 und rechts $190 \mathrm{~nm}$ Breite). Erkennbar ist das schräge Reißen und die Versetzungslinien im Hell- und Dunkelfeld. Die Beugungsbilder zeigen jeweils die (111)-Zonenachsen mit auftretender Überstruktur (markiert mit grünen Kreisen).

Drahtes wieder zusammenfügen. Man erkennt dann, dass auch in diesen Fällen während des Verformung eine geringe Verjüngung um die Bruchstelle aufgetreten ist. In Abbildung 5.28 sind zwei Fälle gezeigt, in denen beide Bruchhälften wieder zusammengefügt wurden, um diese Verjüngung zu veranschaulichen. In dieser Abbildung ist die ursprüngliche Form der Drähte jeweils durch die gestrichelten roten Linien gekennzeich- 
net. Mit Hilfe der grünen gestrichelten Linie ist die Lage einer $\{111\}$-Ebene dargestellt, wie sie in der Projektion in [111]-Richtung sichtbar wäre. Diese Richtung entspricht der Orientierung des Drahtes relativ zum Elektronenstrahl und damit der Bildebene. Die
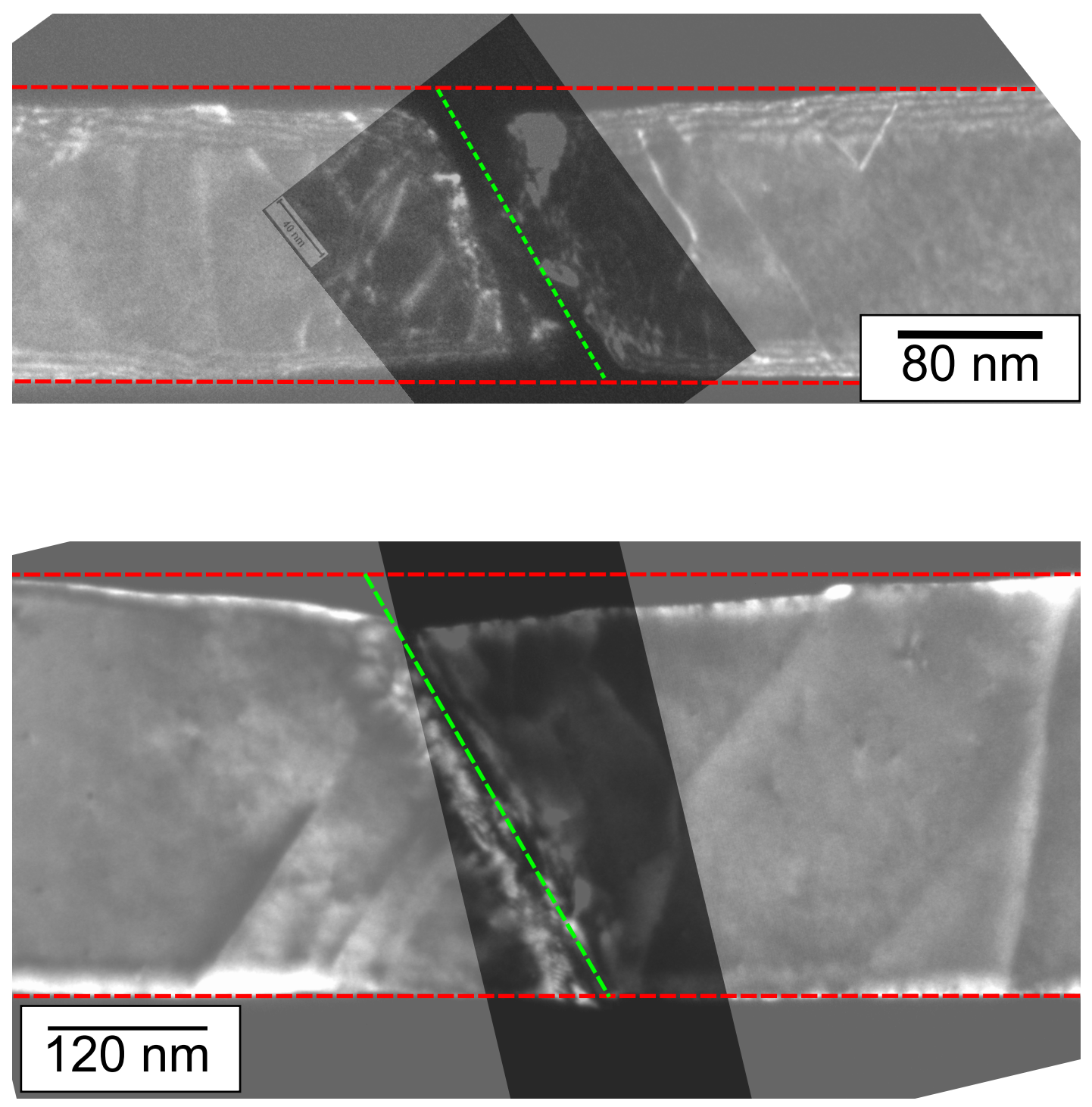

Abbildung 5.28: Verjüngung an Bruchkanten zweier verformter Drähte - Zusammengesetzte Dunkelfeldbilder von zwei getesteten Drähten. Die gestrichelten roten Linien deuten den ursprünglichen Verlauf der Kanten des Drahtes an. Die gestrichelten grünen Linien markieren den Verlauf einer $\{111\}$-Ebene in der gewählten Projektion.

Fläche, die durch die Verjüngung im Vergleich zum Ausgangsdraht an beiden Seiten 


\section{ERGEBNISSE}

fehlt, konnte mit Hilfe einer Bildanalyse Software bei dem $190 \mathrm{~nm}$ breiten Draht zu etwa $\mathrm{A}=7000 \mathrm{~nm}^{2}$ bestimmt werden. Die Dicke des Drahtes kann mit Hilfe des Kontrastes der Facetten $\mathrm{zu} \mathrm{h}=43 \mathrm{~nm}$ abgelesen werden. Nimmt man an, dass das Gesamtvolumen des Drahtes bei der Verformung konstant bleibt, muss das aus den Aufnahmen ermittelte fehlende Volumen vollständig in Dehnung überführt worden sein. Das fehlende Volumen beträgt im ersten Draht

$$
\Delta V=A \cdot h=7000 \mathrm{~nm}^{2} \cdot 43 \mathrm{~nm}=301000 \mathrm{~nm}^{3} .
$$

Der Durchmesser des Drahtes nach der Verformung beträgt $\mathrm{D}_{2}=150 \mathrm{~nm}$ in der Nähe der Bruchkante. Damit muss der Draht um die Länge

$$
\delta L=\frac{\Delta V}{h \cdot D_{2}}=47 \mathrm{~nm}
$$

länger geworden sein. Da die Gesamtlänge des Drahtes etwa $6 \mu \mathrm{m}$ beträgt, entspricht diese Längenänderung demnach einer plastischen Dehnung von $\varepsilon=0,8 \%$.

In dem Draht mit einer Breite von $330 \mathrm{~nm}$ wurde mit dem gleichen Verfahren wie oben beschrieben ebenfalls die Verjüngung um die Bruchstellen ausgemessen (unterer Bild in Abbildung 5.28). Das ermittelte fehlende Volumen ergibt sich unter Berücksichtigung der Dicke von $\mathrm{h}=72 \mathrm{~nm}$ zu

$$
\Delta V=A \cdot h=17300 \mathrm{~nm}^{2} \cdot 72 \mathrm{~nm}=1245600 \mathrm{~nm}^{3} .
$$

Der Enddurchmesser kurz vor dem Reissen ist $\mathrm{D}_{2}=290 \mathrm{~nm}$ an der Bruchkante. Für diesen Fall ergibt sich demnach eine plastische Dehnung von $\varepsilon=1,2 \%$.

Die Dehnungswerte dieser Abschätzungen beziehen sich allerdings nicht auf die Gesamtdehnung des Drahtes, sondern nur auf den Dehnungsteil, der durch die Verjüngung verursacht wird. Es ist anzunehmen, dass gerade im zuletzt betrachteten Fall die Gesamtdehnung deutlich höher ist, da dieser Draht - wie oben beschrieben wurde - einen Verformungsbereich von einigen $\mu \mathrm{m}$ aufweist. Dies deckt sich mit Werten aus quantitativen Tests, in denen eine Gesamtdehnung bis zum Bruch von etwa $2 \%$ ermittelt werden konnte [24].

Die Betrachtung des Abstandes der beiden Bruchteile des Drahtes direkt nach dem Bruch lässt eine grobe Abschätzung der wirkenden Spannung zu. Dies ist möglich, wenn man davon ausgeht, dass der Draht direkt nach dem Reißen ausschließlich den 
elastischen gedehnten Teil relaxiert und sich der CuBe-Rahmen ideal steif verhält. Ausserdem muss gesichert sein, dass sich der Draht auf seiner gesamten Länge auf einem gleichen Spannungsniveau befindet. Dies war für den Draht mit 240 nm Durchmesser näherungsweise der Fall. Abbildung 5.29 zeigt ein Einzelbild aus dem Video direkt nachdem der Bruch eintrat. In diesem Zustand lässt sich der Abstand $\Delta \mathrm{l}$ zwischen den

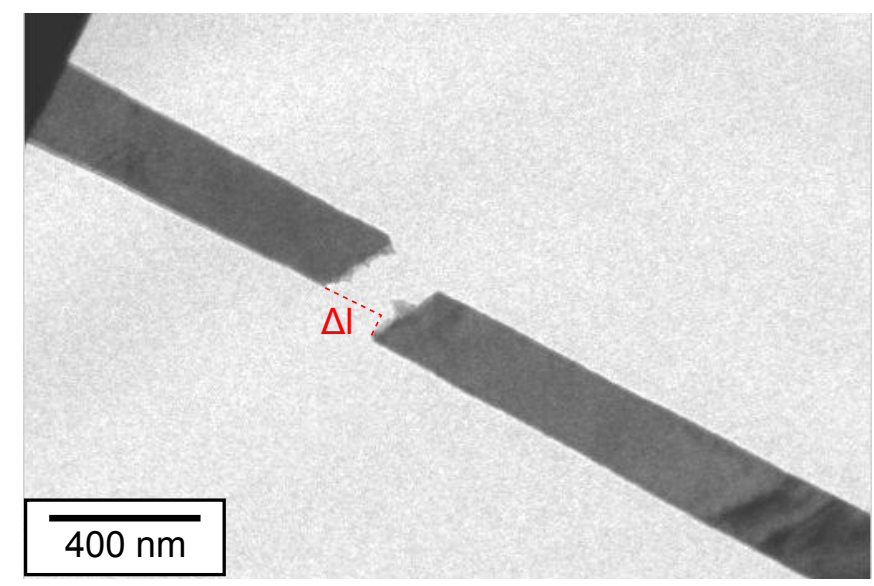

Abbildung 5.29: Übersichtsbild eines gerissenen Drahtes - Dieses Hellfeldbild des 240 nm Drahtes zeigt den Abstand der Bruchkanten im ersten Einzelbild nach dem Bruch. Durch das Ausmessen des Abstandes kann man auf die Spannung vor dem Bruch zurückschließen.

beiden Enden ausmessen. Er beträgt hier etwa 160 nm, was bei einer Drahtlänge von $5 \mu \mathrm{m}$ einer Dehnung von 3,2\% entspricht. Fasst man dies als die maximal mögliche elastisch Dehnung auf, erhält man unter Verwendung des Hook'schen Gesetzes die dafür nötige Spannung beim Moment des Bruches. Sie beträgt

$$
\sigma_{\text {Bruch }}=\varepsilon \cdot E=\frac{\Delta l}{5 \mu \mathrm{m}} \cdot 79 \mathrm{GPa}=2,5 \mathrm{GPa} .
$$

Dieser Wert ist deutlich höher als für makroskopische Proben mit Korndurchmessern von einigen $\mathrm{mm}$ bis $500 \mathrm{~nm}$ erwartet wird. Dort werden typischer Weise als Fließspannung Werte zwischen 15 und 125 MPa berichtet [45]. Erst in Proben mit Dimensionen von einigen Nanometern zeigen sich Fließspannungen in der hier auftretenden Größenordnung [46, 47].

Dennoch ist der hier ermittelte Wert für die Bruchspannung eher als Abschätzung der Größenordnung denn als Messung zu verstehen. Durch eine Fehlorientierung der Drahtenden aus der Bildebene heraus ist es leicht möglich, den gemessenen Abstand zu 


\section{ERGEBNISSE}

beeinflussen. Diese Fehlorientierung tritt häufig dadurch auf, dass die ungewollte Platinabscheidung während des Transferprozesses bevorzugt auf der dem Elektronenstrahl zugewandten Seite geschieht. Durch diese unsymmetrische Verteilung erhält man eine Bimetall-ähnliche Struktur, die sich schon bei leichter Erwärmung verbiegen kann. Liegt der Draht zusätzlich mit einer Facette nicht genau auf dem Rahmen sondern leicht verkippt, kann diese Verbiegung zu einer seitlichen Verschiebung der Drahtenden führen, wie in Abbildung 5.29 zu erkennen ist. Dies führt in jedem Fall dazu, dass der Abstand als zu groß angenommen und dadurch die Spannung überschätzt wird.

\subsubsection{Defektdichte}

Die Dichte an gespeicherten Versetzungen wurde an dem Draht mit der Breite von 330 nm ausgemessen. Dazu ist der Draht im Hellfeld nach dem Reißen abschnittweise abgebildet und jedes sichtbare Versetzungssegment in seiner Länge ausgemessen worden. Da bei gebogenen Linienlementen die Kenntnis über die Gleitebene verloren geht, wurden die einzelnen Längen ohne Berücksichtigung der Projektion aufsummiert. Mit diesem Verfahren konnte eine untere Grenze der Gesamtlänge von $\mathrm{L}_{\text {Gesamt }}=6,8 \mu \mathrm{m}$ ermittelt werden. Das Volumen des Drahtes beträgt wegen des rhombischen Querschnitts

$$
V=150 \mathrm{~nm} \cdot 43 \mathrm{~nm} \cdot 6000 \mathrm{~nm} \cdot \sin \left(70,53^{\circ}\right)=0,036 \mu \mathrm{m}^{3}
$$

Damit erhält man eine Versetzungsdichte von

$$
\rho=\frac{L_{\text {Gesamt }}}{V}=1,86 \cdot 10^{10} \frac{1}{\mathrm{~cm}^{2}}
$$

Dieses Ergebnis zeigt deutlich, dass es in diesen Experimenten möglich war Defekte in kleinskaligen Proben zu speichern. Die Aussage dieser Beobachtung wird deutlich, wenn man andere Experimente vergleichend hinzuzieht. Diese zeigen, dass es zwar möglich ist, hohe Versetzungesdichten in Proben zu erreichen, deren Mikrostruktur Längen von einigen Nanometern aufweist [48]. In diesen ist die Versetzungsbewegung allerdings durch innere Grenzflächen eingeschränkt. Bei Proben, die nur durch freie Oberflächen begrenzt werden,ergibt sich ein anderes Bild. Dort wird beobachtet, dass nach erfolgter Verformung selten Defekte gespeichert werden [49, 50]. Nur in Ausnahmefällen wird berichtet, dass Versetzungen im Material gespeichert bleiben. Dies kann aufgrund der komplexen Geometrie [51] geschehen oder weil Versetzungen aufgrund der Lage zur 
Belastungsrichtung keine Kraft spüren [7]. Sind in freistehenden Proben einfach aktivierbare Quellen für die Versetzungsnukleation vorhanden, sind auch Dichten von bis zu $6 \cdot 10^{9} 1 / \mathrm{cm}^{2}$ beobachtet worden [52]. Der direkte Vergleich dieses Wertes mit der in diesem Versuch auftretenden Dichte zeigt, dass hier auch ohne erkennbar aktive Quelle ähnliche Dichten erreicht werden konnten. Vor diesem Hintergrund wird klar, dass die hohe Defektdichte und die homogene Verteilung von Versetzungen einer ausführlicheren Betrachtung in Kapitel 6 bedarf.

\subsection{Einfluss von FIB-Schädigung auf den Verformungs- mechanismus}

Wie Richter et. al in ihrer Veröffentlichung [34] und die eigene Vorcharakterisierung aus Abschnitt 4 gezeigt haben, weisen die verwendeten Nanodrähte eine außerordentlich hohe kristalline Perfektion auf. Inbesondere konnten weder Oberflächendefekte wie Rauheit noch Volumendefekte gefunden werden. Inwieweit der beobachtete Defekttyp aus dem Abschnitt 5.1 und die Defektstruktur durch eine schadhafte Oberfläche verändert wird, sollte in einem eigenständigen Experiment untersucht werden, bei dem die vorhandene Perfektion gezielt beschädigt wurde.

In Abschnitt 5.1 konnte gezeigt werden, dass Stapelfehler, die wahrscheinlich durch die Nukleation und Bewegung von Partialversetzungen entstehen, einen wesentlichen Beitrag zur Verformung der Drähte haben, sofern sie nicht breiter sind als etwa 180 nm. Zwei Nanodrähte mit 65 und $105 \mathrm{~nm}$ Breite wurden daher gezielt mit einer definierten Dosis Ga-Ionen im FIB beschossen. Durch die hohe kinetische Energie bei 30 $\mathrm{kV}$ Beschleunigungsspannung und die Atommasse von 69,7 u der auftreffenden Ionen finden verschiedene Prozesse im Material statt [53], die die Proben in ihrer Perfektion beeinflussen. Die für das Experiment entscheidenden Wechselwirkungen sind:

1. Erzeugung von Leerstellen und Leerstellen-Agglomeraten

2. Implantation von Gallium-Ionen in das Ausgangsmaterial

3. Abtragen von Gitteratomen.

Von allen diesen Erscheinungen ist bekannt, dass sie einen Einfluss auf die mechanischen Eigenschaften der Probe haben. Insbesondere eine geschädigte Oberfläche [1] und Ausscheidungen [54] werden in der Literatur in diesem Zusammenhang diskutiert. 


\subsubsection{Präparation und Galliumkonzentration}

Das FIB ermöglicht die genaue Einstellung der Gallium-Ionen-Dosis über einen wohl definierten Bereich. Um größtmögliche Vergleichbarkeit zwischen den beiden Versuchen zu erreichen wurde dieser Parameter konstant gehalten. Die Dosis betrug in beiden Fällen $\mathrm{D}=7,02 \mathrm{pC} / \mu \mathrm{m}^{2}$. Die Angabe dieser Dosis bezieht sich dabei aber auf eine senkrecht zum Ionenstrahl stehende Probenoberfläche. Die für diesen Versuch verwendete Geometrie ist in Abbildung 5.30 dargestellt. Der Ionenstrahl trifft bei diesem Aufbau -

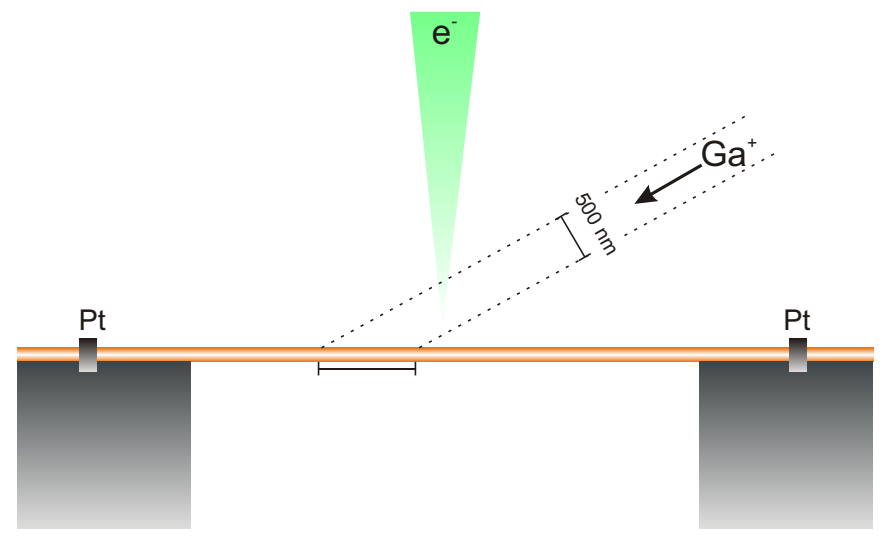

Abbildung 5.30: Skizze des Ga-Implantationsexperimentes - Der Ga-Ionenstrahl trifft unter einem Winkel von $52^{\circ}$ zentral auf die Oberfläche des Drahtes und erzeugt dort ein lokale Veränderung.

bedingt durch die Anordnung der Quellen am Gerät - unter einem Winkel von $52^{\circ}$ auf die Probe. Damit wird die eingestelle Dosis D auf eine größere Fläche verteilt. Korrigiert man diese geometrischen Zusammenhänge erhält man eine effektive Dosis von

$$
D_{\text {effektiv }}=D \cdot \cos 52^{\circ}=4,32 \mathrm{pC} / \mu \mathrm{m}^{2}
$$

Die Gesamtanzahl der auf den Draht gelangten Ionen ist noch von der Drahtdicke abhängig. Die Bereiche hatten jeweils gleiche Längen auf dem Draht von 812 nm. Dies bedeutet, dass im Falle des dünneren Drahtes eine Fläche von

$$
A_{1}=\frac{500 \mathrm{~nm}}{\cos 52^{\circ}} \cdot 65 \mathrm{~nm}=52788 \mathrm{~nm}^{2} \approx 0,053 \mu \mathrm{m}^{2}
$$

bestrahlt wurde. Die Gesamtladung der auf dem Draht eintreffenden Ionen ist somit

$$
Q_{1}=A_{1} \cdot D_{\text {effektiv }}=0,23 \mathrm{pC}
$$


Für den Draht mit einer Breite von 105 nm gilt analog

$$
A_{2}=\frac{500 \mathrm{~nm}}{\cos 52^{\circ}} \cdot 105 \mathrm{~nm}=52788 \mathrm{~nm}^{2} \approx 0,085 \mu \mathrm{m}^{2}
$$

und damit für die Ladung

$$
Q_{2}=A_{2} \cdot D_{\text {effektiv }}=0,37 \mathrm{pC} .
$$

Die durch diese Rechnungen ermittelten Ladungen können in Ionenanzahl umgerechnet werden und mit der Gesamtzahl der Atome im bestrahlten Bereich verglichen werden. Die Dicke der Drähte konnte in diesem Versuch nicht bestimmt werden, sodass für die Abschätzung ein runder Drahtquerschnitt mit der jeweiligen Breite als Durchmesser angenommen wird. Der prozentuale Anteil berechnet sich dann nach der Vorschrift

$$
c[a t . \%]=\frac{N_{G a}}{N_{A u}}=\frac{Q_{i} / e}{N_{A u}}
$$

Die Anzahl der Goldatome $\mathrm{N}_{A u}$ lässt sich über das Volumen der Einheitszelle berechnen, diese hat 4 Atome in einem Volumen der Größe von $\mathrm{a}^{3}$. Damit befinden sich in dem idealisierten runden Draht

$$
N_{A u}=\frac{\pi \cdot d^{2} \cdot 812 \mathrm{~nm}}{a^{3}}=1,6 \cdot 10^{8}
$$

Atome. Setzt man die beiden Zahlen $\mathrm{N}_{A u}$ und $\mathrm{N}_{G a}$ in Verhältnis zueinander, erhält man

$$
c_{1} \approx 0,9 \text { at. } \% \text {. }
$$

Für den Draht mit einem Durchmesser von 105 nm ergibt sich nach analoger Rechnung eine Konzentration von

$$
c_{2} \approx 0,6 \text { at. } \% \text {. }
$$

Bei dieser Abschätzung wurde angenommen, dass alle auftreffenden Ionen im Material des Drahtes vollständig implantiert und homogen verteilt werden. Allerdings beträgt die Eindringtiefe von Gallium in Gold bei $30 \mathrm{kV}$ nur einige $10 \mathrm{~nm}$. Dies kann mit Hilfe einer SRIM (The Stopping and Range of Ions in Matter)[55] Simulation gezeigt werden. Um die experimentellen Bedingungen so weit wie möglich nachzustellen, wurden als Eingangsparameter für diese Berechnung Ionen mit einer kinetischen Energie von $30 \mathrm{keV}$ gewählt. Zudem treffen diese wie auch im Experiment unter einem Winkel von $52^{\circ}$ auf die Oberfläche des gewählten Ziels Gold auf. Die Stoßkaskaden der einzelnen 


\section{ERGEBNISSE}

Ionen werden dann mit Hilfe einer Monte Carlo Simulation berechnet (siehe Abbildung 5.31) und liefern eine Aussage über die sich ergebende Verteilung und Eindringtiefen. Aus den errechneten Verläufen der Stöße mit dem Gold wird deutlich, dass sich der
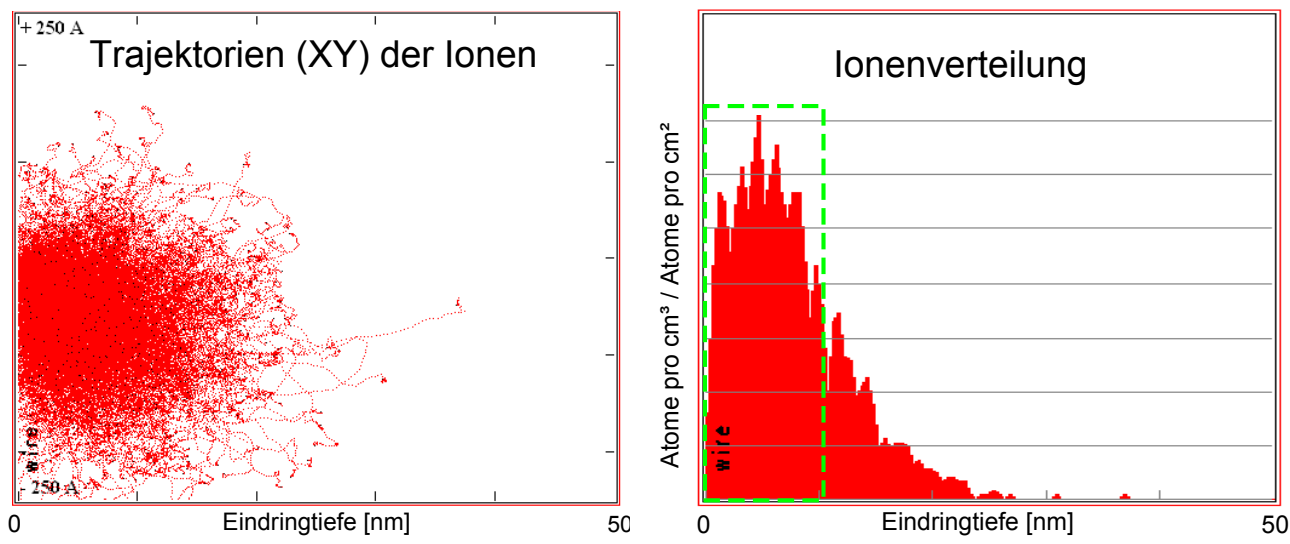

Abbildung 5.31: SRIM Simulation zu Gallium in Gold - Im linken Teil des Bildes sind die errechneten Trajektorien von Ga-Ionen in Gold zu sehen. Rechts ist dargestellt, welche Tiefenverteilung sich daraus ergibt.

Großteil des Galliums in einem Bereich an der Oberfläche bis in eine Tiefe von 10 $\mathrm{nm}$ verteilt. Dies ist durch den Kasten im rechten Teilbild in Abbildung 5.31 deutlich gemacht worden. In einer Tiefe über $35 \mathrm{~nm}$ sind nur noch sehr vereinzelt Ionen anzutreffen. Da die so ermittelte Eindringtiefe deutlich kleiner ist als die Dicke der bestrahlten Drähte, ist davon auszugehen, dass die Konzentration an der dem Ionenstrahl zugewandten Oberseite deutlich höher ist als auf der abgewandten Seite. Um dies zu quantifizieren kann man die Anzahl der Ionen aus den obigen Berechnungen ins Verhältnis setzen zu einem Volumen aus Gold, dass nur $10 \mathrm{~nm}$ Dicke aufweist. Damit ist die Dicke des Drahtes nicht mehr relevant für die nun gleichen Konzentrationen. Diese betragen daher in beiden Fällen

$$
\begin{aligned}
& c_{1}=4,5 \text { at. } \% \\
& c_{2}=4,5 \text { at. } \%
\end{aligned}
$$

Diese Angaben lassen einen Vergleich mit dem Phasendiagramm von Gold und Gallium zu, wie es in Abbildung 5.32 gezeigt ist. Zusätzlich eingezeichnet sind mit roter Markierung die Bereiche der hier abgeschätzten Galliumkonzentrationen. Man erkennt hier, dass bei der errechneten Konzentration von 0,6 at.\% noch ein Ein-Phasen-Gebiet 
vorliegt und die Galliumatome im Wirtsgitter gelöst werden können. Bei der realistischeren Abschätzung der Konzentration von 4,5 at. \%, die auch die Eindringtiefe in Gold berücksichtigt, wechselt man im Phasendiagramm in ein Zweiphasengebiet aus Gold und der $\beta^{\prime}$-Phase. Dies bedeutet, dass das Gallium nicht mehr im Gitter gelöst werden kann, sondern höherkonzentrierte Ausscheidungen der $\beta^{\prime}$-Phase in der Goldmatrix bildet.

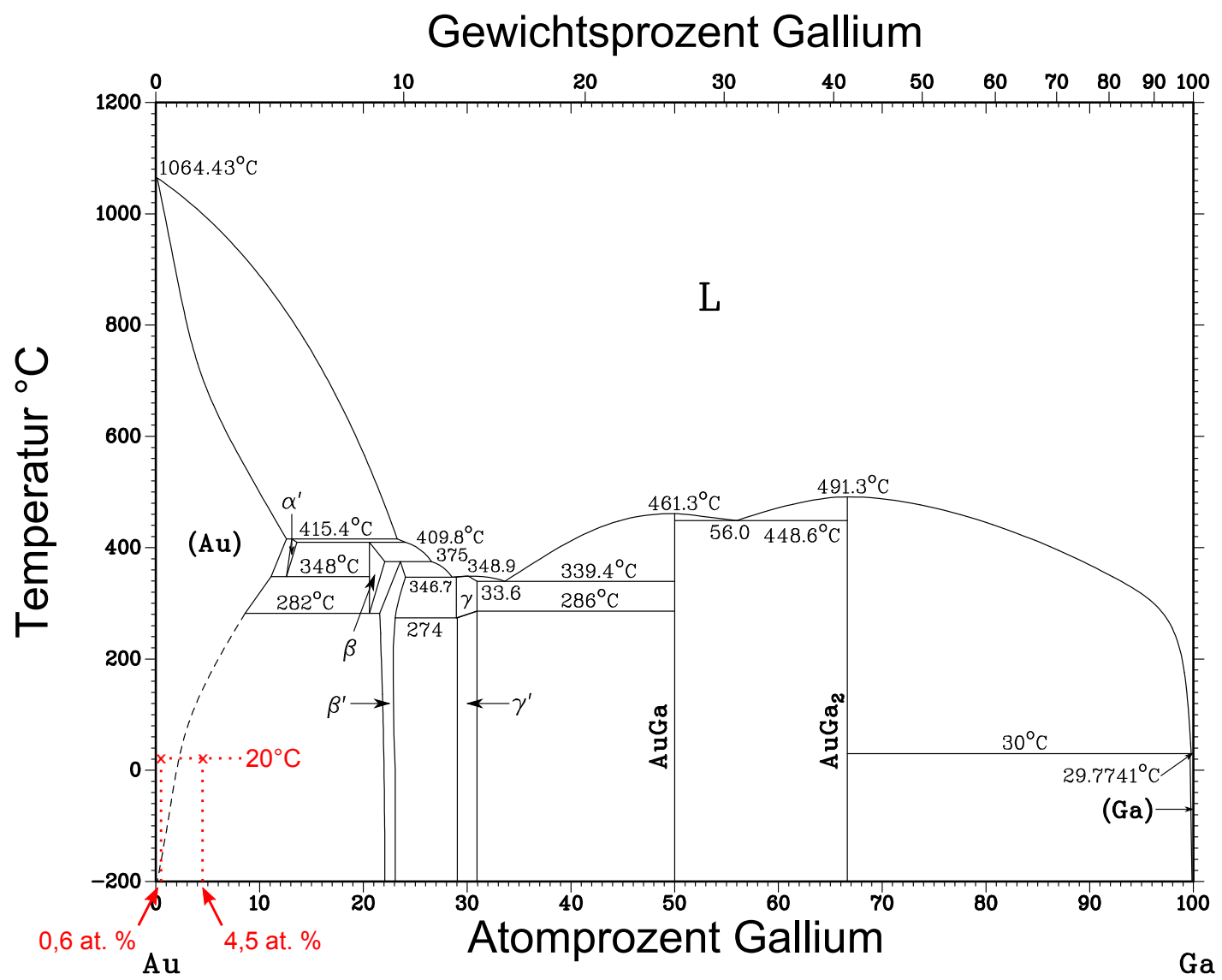

Abbildung 5.32: Gold-Gallium Phasendiagramm - Binäres Phasendigramm von Gold und Gallium. Eingezeichnet ist der errechnete Konzentrationsbreich für die beiden bestrahlten Drähte. Diese liegen zwischen 0,6 und 4,5 at.\% Gallium. 


\subsubsection{Verformungsverhalten der geschädigten Drähte}

Nach der oben beschriebenen Vorpräparation sind beide Drähte unter vergleichbaren Bedingungen wie die in Abschnitt 5.1 getesteten Proben gezogen worden. Die Voruntersuchung unter $\sigma=0$ zeigte, dass in dem Einflussbereich des Galliums eine deutliche Kontraständerung im Draht zu erkennen war. Der übliche homogene Kontrast entlang des Drahtes ist nun verschwunden und die Oberfläche zeigt fleckige hell-dunkel Änderungen im Größenbereich von einigen Nanometern (siehe Abbildung 5.33). Die genaue Art der hier vorliegenden Defekte kann in dem gewählten Aufbau nicht sicher bestimmt werden.

An der Oberfläche der Drähte ist ersichtlich, dass der Abtrageprozess nicht nur in der Platinschicht stattgefunden, sondern auch die eigentliche Oberfläche des Drahtes beschädigt hat. Eine besonders ausgeprägte Form ist in Abbildung 5.33 dargestellt. Dort ist der Übergangsbereich zwischen dem Teil, der mit Ionen bestrahlt wurde, und dem Ausgangszustand gezeigt. Die gestrichelten Linien deuten dabei den ursprünglichen Verlauf der Oberfläche an. Hieraus wird ersichtlich, dass durch die Abtragung eine lokale Verjüngung des Drahtes entsteht. Für den Zugversuch bedeutet dies, dass eine höhere Spannung in diesem Bereich vorliegt.

Während des Zugversuches entstehen zuerst innerhalb des bestrahlten Bereiches einzel-

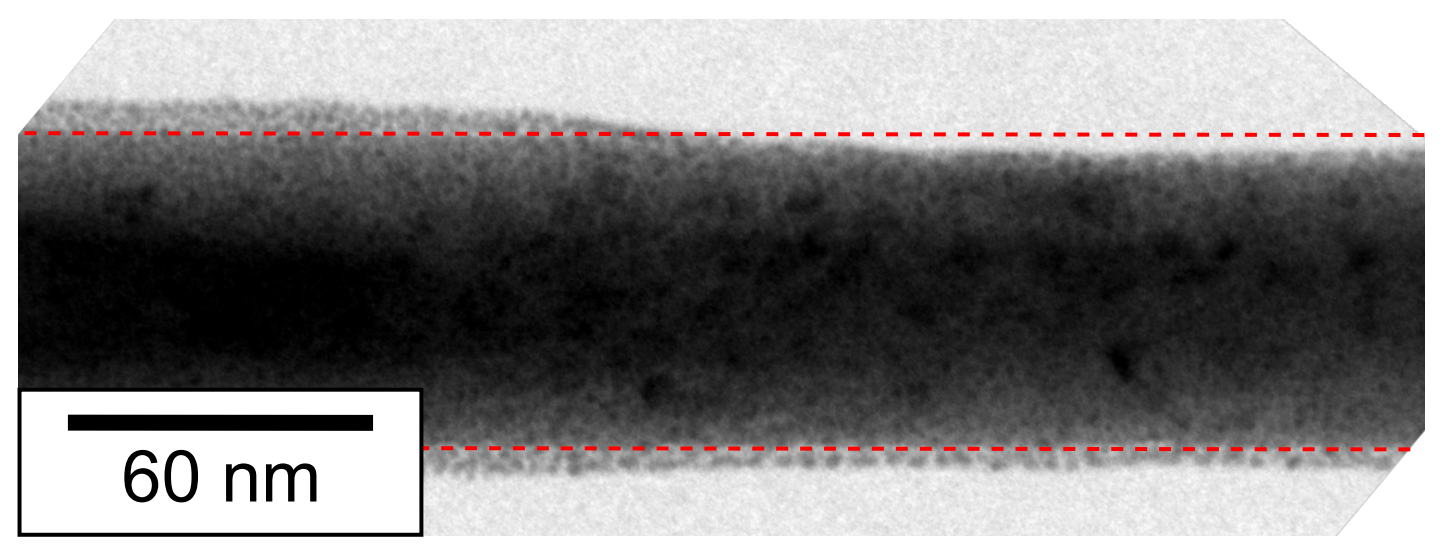

Abbildung 5.33: Gallium-Schädigung - Übergangsbereich des $65 \mathrm{~nm}$ Drahtes. Die Verjüngung des Drahtes im rechten Teil des Bildes ist durch die Bestrahlung mit Ga-Ionen und die dadurch verbundene Abtragung entstanden.

ne planare Defekte, deren oszillierender Kontrast vergleichbar ist mit dem der beobachteten Stapelfehler aus 5.1. Deutlich erkennbar ist allerdings, dass nicht mehr die scharf 
abgegrenzten Kontraste der planaren Defekte vorliegen, sondern diese durch Überlagerung von punkthaften Flecken in ihrem Erscheinungsbild beeinträchtigt werden. Diese Art von Defekten ist aus TEM Untersuchungen von bestrahlten Proben bekannt und ist auf die Schädigung durch die Ionen zurückzuführen [56].

Im weiteren Verlauf ist im Beugungsbild eine zunehmende Verzwillingung im Beugungsbild erkennbar. Beide Defektarten sind beispielhaft in beiden Drähten in Abbildung 5.34 gezeigt. Später entstehen auch planare Defekte im nicht bestrahlten Bereich, die

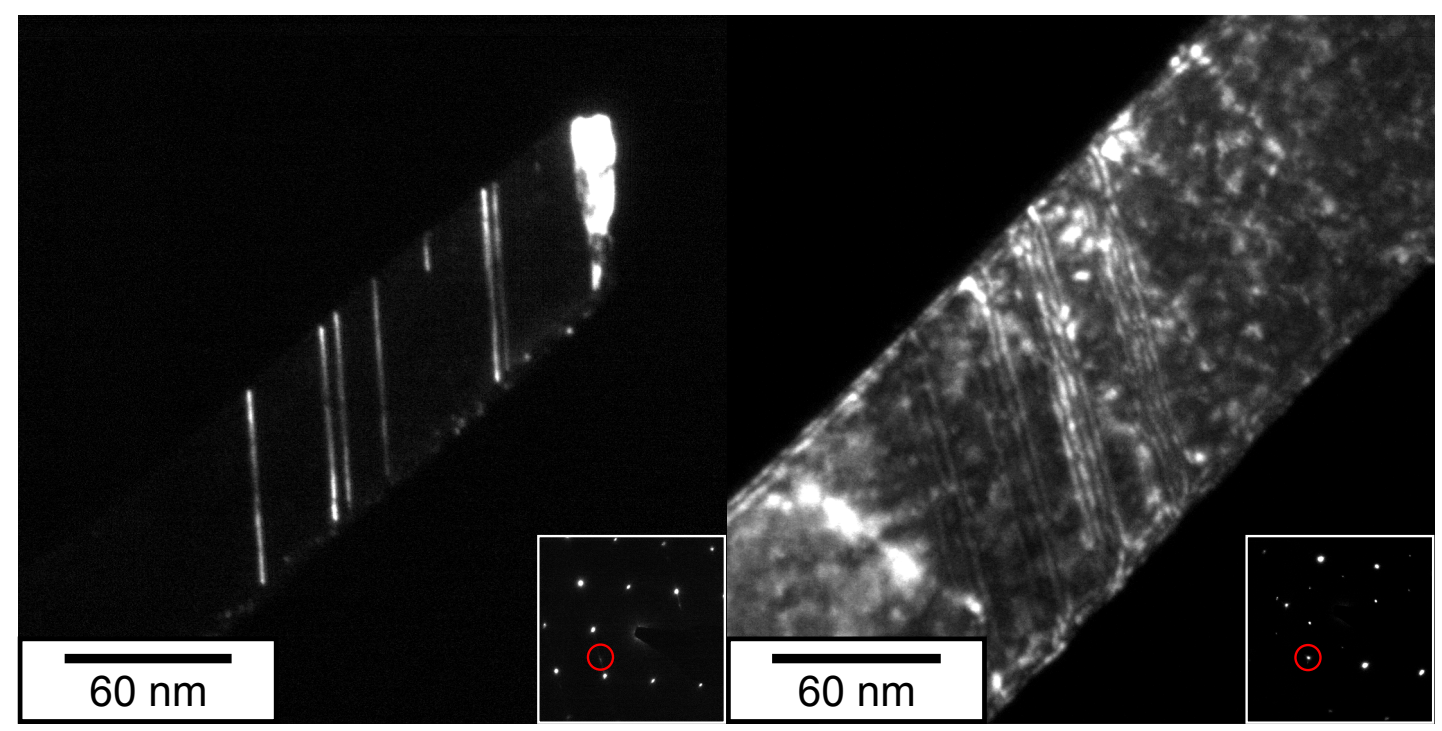

Abbildung 5.34: Defekte und Bruchkante bestrahlter Drähte - Im linken Teilbild ist ein Dunkelfeldbild einer Bruchkante mit Zwilling in einem $65 \mathrm{~nm}$ Draht gezeigt. Im rechten Teilbild sind typische Defekte mit oszillierendem Kontrast in dem $105 \mathrm{~nm}$ Draht im Dunkelfeld gezeigt.

Defektdichte und Aktivität ist dabei aber innerhalb des bestrahlten Bereiches höher. Darüber hinaus ist aus der Videoaufnahme ableitbar, dass der Großteil der Verformung und der anschließende Bruch innerhalb des verjüngten Bereiches stattfindet.

Zusammenfassend kann man folgern, dass trotz der FIB-Schädigung in den beiden Drähten derselbe Defekttyp aufgetreten ist, wie in vergleichbar großen ungeschädigten Drähten. Wie zu erwarten war fand sowohl der Bruch, als auch der Großteil der Verformung innerhalb des bestrahlten Bereiches statt, weil dort mit einer Spannungskonzentration aufgrund der Verjüngung zu rechnen war. Bemerkenswert bleibt das Auftreten von Stapelfehlern auch außerhalb des bestrahlten Bereiches. Es kann daher vermutet 


\begin{tabular}{ccc}
$\begin{array}{c}\text { Drahtbreite } \\
{[\mathrm{nm}]}\end{array}$ & $\begin{array}{c}\text { Drahtlänge } \\
{[\mathrm{nm}]}\end{array}$ & Besonderheiten \\
\hline \hline 70 & 2300 & Zwilling entlang der Drahtachse \\
110 & 3000 & - \\
260 & 3600 & flacher Draht \\
\hline \hline
\end{tabular}

Tabelle 5.3: Übersicht über die Biegeversuche - Diese Aufstellung zeigt alle in Biegegeometrie verformten Drähte. In einem Fall lag eine Besonderheit in Form eines Zwillings vor. Der letzte getestete Draht hatte eine plattenartige Gestalt

werden, dass die Verjüngung keinen so starken Effekt auf die Spannung hat, dass eine Verformung nur dort stattfindet.

\subsection{Biegeversuche}

Wie in Kapitel 4 erläutert wurde, sind an denselben Drähten zusätzlich zu uniaxialen Zugversuchen auch Biegeversuche durchgeführt worden. Dabei wurden 3 Drähte mit Breiten zwischen 70 und $260 \mathrm{~nm}$ getestet. Tabelle 5.3 fasst die Eigenschaften der Drähte und deren Besonderheiten zusammen.

Der erste getestete Draht mit einer Breite von $70 \mathrm{~nm}$ wies vor der Verformung keinerlei Defekte auf. Allerdings lag wie zuvor in Tabelle 5.3 angemerkt in seiner Mikrostruktur eine Besonderheit vor. Entlang der Drahtachse war dieser Draht verzwillingt. Dieses konnte durch die Analyse des Beugungsbildes und entsprechende Dunkelfeld-Bilder nachgewiesen werden (Abbildung 5.35).

Um ein Gefühl dafür zu erlangen, wie weit der Draht verbogen werden muss, um plastisch zu verformen, wurde die Belastung in mehrere ansteigende Schritte aufgeteilt. Dazu wurde die abgeflachte Spitze im STM-Halter mit der Feinbewegung der Piezos langsam parallel zur Drahtachse auf diesen zu bewegt und wieder entfernt. Nach dem jeweiligen Belastungsschritt wurde mit Hell- und Dunkelfeldaufnahmen kontrolliert, ob der Draht Defekte aufwies oder sich verformt hatte. Zusätzlich wurde mit der CCD Kamera von jedem Belastungsschritt ein Video aufgezeichnet. Als experimentell nachteilig wirkt sich aus, dass durch die Verbiegung des Drahtes während des Versuches keine gleichbleibende Abbildebedingung zu realisieren war.

Die erste sichtbare plastische Verformung konnte in diesem Draht im Dunkelfeldbild auf- 


\subsection{Biegeversuche}
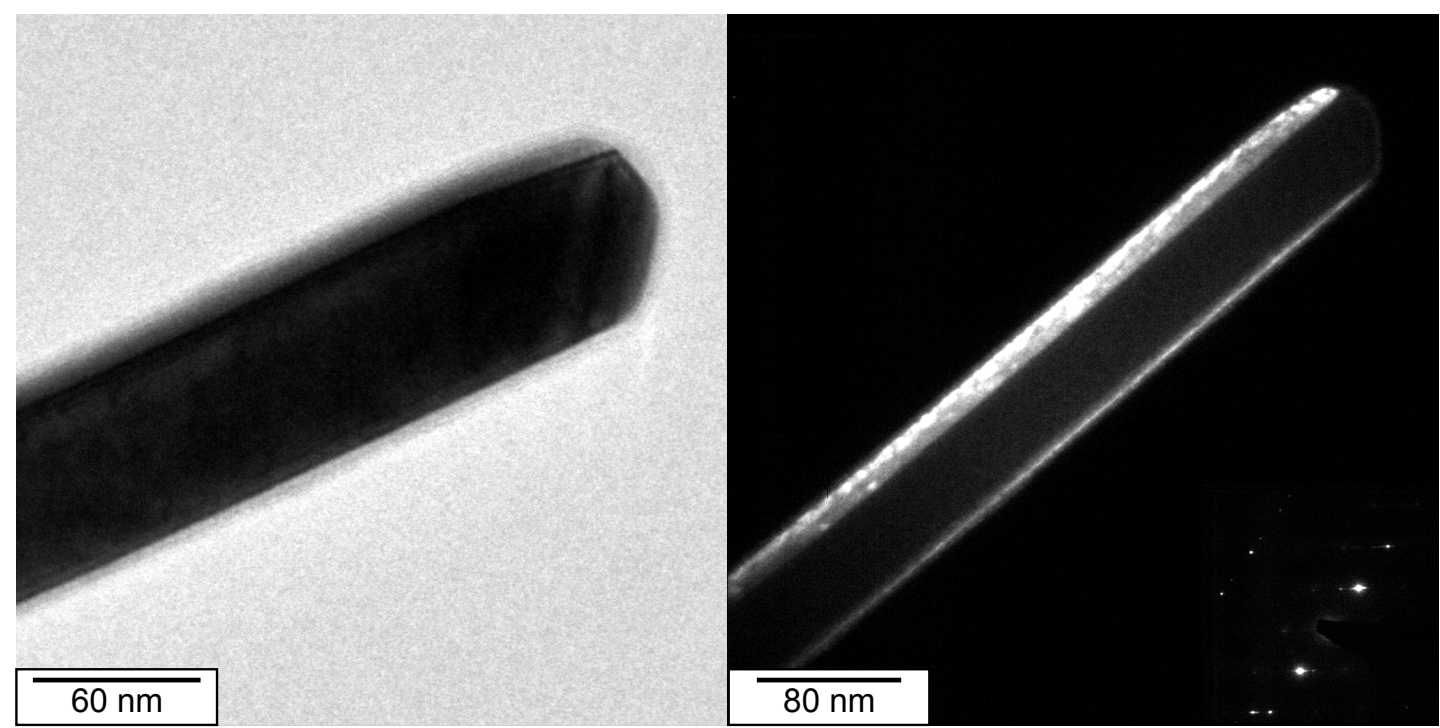

Abbildung 5.35: Mikrostruktur des Drahtes beim ersten Biegeversuch - TEM Hellfeld- (links) und Dunkelfeldaufnahmen (rechts) zeigen die Verzwilligung des Drahtes entlang der Drahtachse.

gezeichnet werden. Man erkennt dort trotz variabler Abbildebedingung deutlich, dass in einem Einzelbild des Videos sich der Kontrast lokal und abrupt ändert. Diese Änderung kann mit einer Veränderung des Spannungszustandes und damit der Nukleation eines Defektes erklärt werden. An diesem Punkt wurde die Verformung abgebrochen und der Draht wieder entlastet. Nach der Entlastung ist eine bleibende Verformung in Form eines Knicks im Draht erkennbar. Diese Formänderung ist in Abbildung 5.36 b) gezeigt. Die entstandenen Defekte konnten allerdings durch eine ungünstige Orientierung im TEM nicht genauer untersucht werden.

Der Moment, an dem die erste sichtbare Nukleation eines Defektes erkennbar war, lässt jedoch eine Abschätzung der Fließspannung zu. Abbildung 5.36 zeigt den Vergleich des Drahtes ohne Kontakt zur Spitze und im Punkte der beobachteten Kontraständerung. Eine Längenmessung des Drahtes im Ausgangszustand wie auch im gebogenen Zustand liefert gleiche Werte, sodass gefolgert werden kann, dass der Draht in beiden Situationen in der Bildebene liegen muss, damit ist aus dem TEM Bild der Biegeradius direkt ablesbar. In dem hier gezeigten Fall beträgt der Radius $\mathrm{r}=713 \mathrm{~nm}$. Der Drahtdurchmesser betrug $70 \mathrm{~nm}$. Aus der Gleichung

$$
\varepsilon=\frac{\Delta l}{l}=\frac{U_{Z u g}-U_{0}}{U_{0}}=\frac{2 \pi(r+d / 2)-2 \pi r}{2 \pi r}=\frac{2 \pi d / s}{2 \pi r}=\frac{d / 2}{r}
$$




\section{ERGEBNISSE}

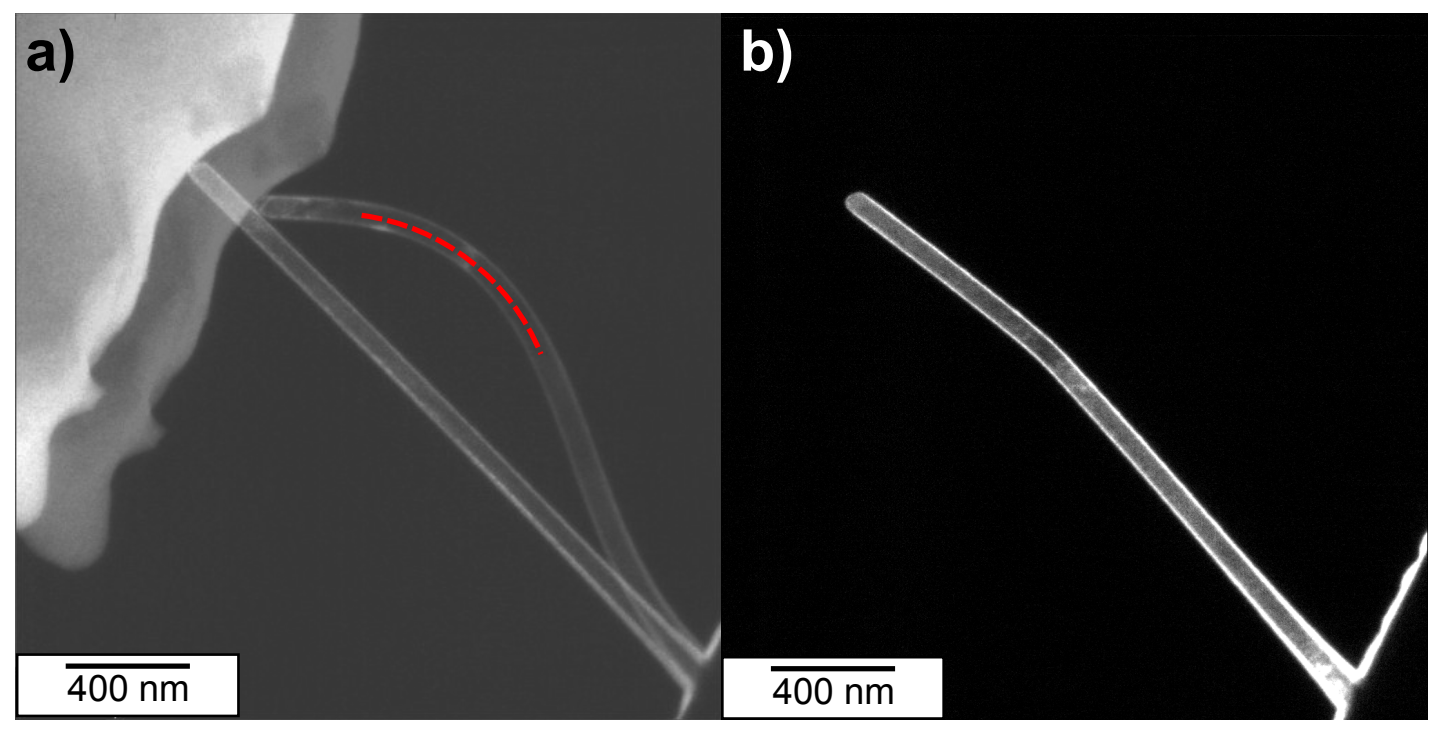

Abbildung 5.36: Quantitative Abschätzung der Spannung - a) Zusammengesetztes TEM Dunkelfeldbild, das den Ausgangszustand und die Verbiegung zeigt, bei der die erste Nukleation beobachtet wurde. Eingezeichnet ist mit rot gestrichelter Linien der ermittelte Biegeradius über den die maximale Spannung berechnet werden. b) Dunkelfeldaufnahme, der bleibenden plastischen Verformung nach Beobachtung der ersten Defektnukleation.

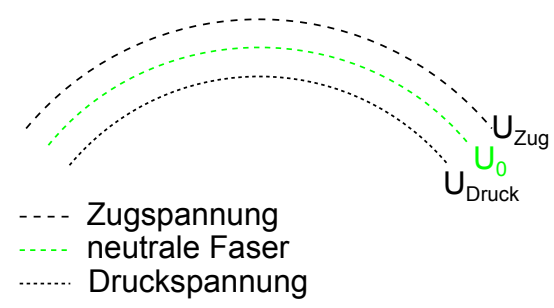

Abbildung 5.37: Schemazeichung der Verbiegung - Im Aussenbereich des Drahtes liegt eine Zugspannung vor, die zu einer Dehnung von 0,049 führt. Im Innenbereich wird der Draht mit gleicher negativer Dehnung gestaucht

lässt sich die Dehnung in der äußersten Faser des Drahtes berechnen. Die Bezeichnungen in der Formel folgen dabei der Skizze 5.37. Mit den gemessenen Werten ergibt sich somit eine maximale Dehnung von $\varepsilon=0,049$. Nach

$$
\sigma=\varepsilon \cdot E_{A u}=4,9 \% \cdot 79 \mathrm{GPa}=3,9 \mathrm{GPa}
$$

liegt damit eine Spannung von etwa 3,9 GPa im Außen- und Innenbereich der Biegung vor. Diese kann als nötige Spannung für die Versetzungsnukleation verstanden werden. 
Dabei bleibt aber unklar, wie groß die Spannung auf dem Gleitsystem war und ob die Versetzung auf der Druck- oder der Zugseite nukleiert wurde.

In einem zweiten Versuch wurde ein weiterer Draht mit einer Breite von $110 \mathrm{~nm}$ getestet, der keine Verzwillingung entlang der Drahtachse aufwies. In diesem Fall lag die Richtung der Verbiegung allerdings nicht in der Bildebene. Dies wird in Abbildung 5.38 deutlich, wo der Ausgangszustand zusammen mit der maximalen Verformung dargestellt ist. Die auf die Bildebene projizierte Länge ist deutlich kürzer, was den Rückschluss zulässt, dass die Verbiegung im Wesentlichen aus der Bildebene heraus stattfindet. Somit ist keine quantitative Abschätzung der Spannung möglich, wie es beim ersten Draht der Fall war. Nach erfolgreicher Verformung ist lokal an einer Stelle im Draht eine hohe
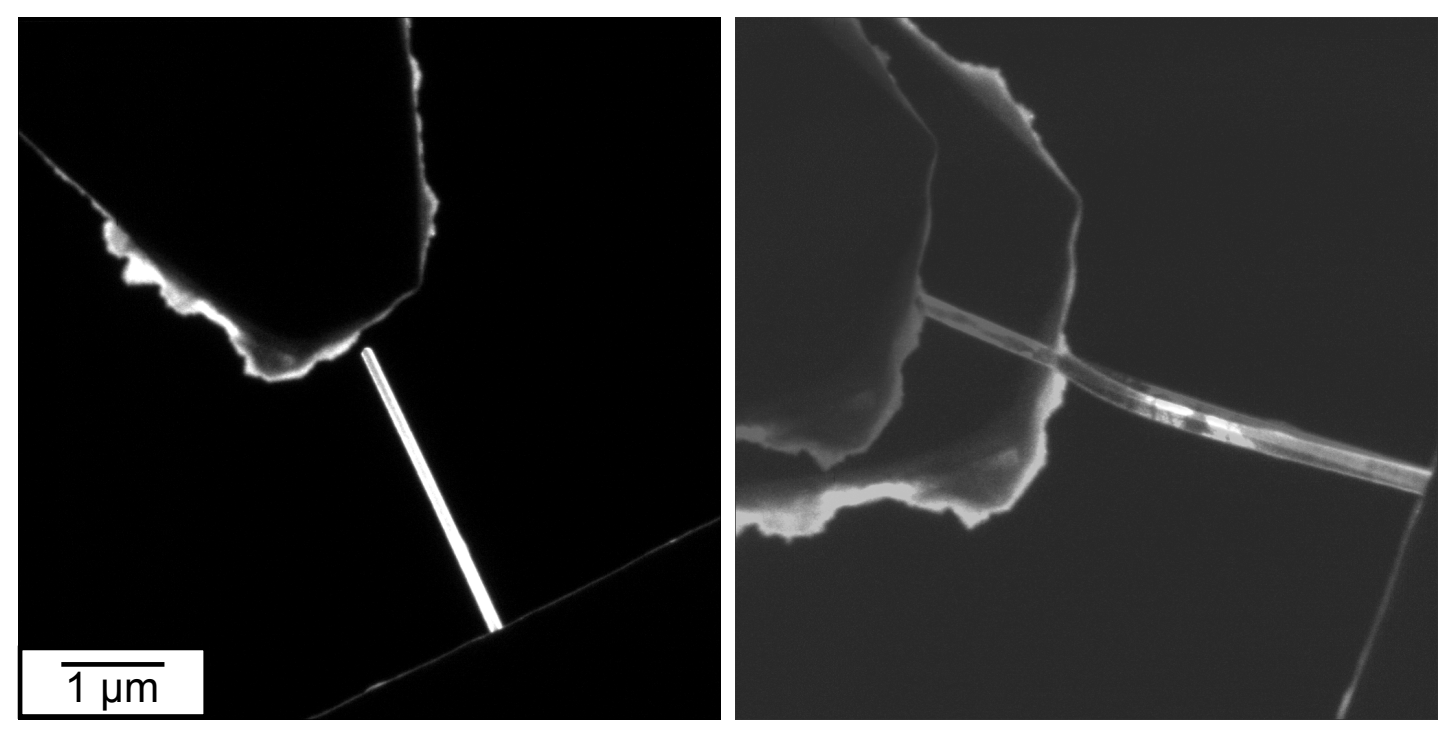

Abbildung 5.38: Der zweite Biegeversuch - TEM Dunkelfeldaufnahmen der Testgeometrie und Länge des $110 \mathrm{~nm}$ Drahtes im Ausgangszustand (links). Die Überlagerung von unverformten und verformten Drahtes zeigt, dass die Verbiegung nicht in der Bildebene erfolgt (rechts).

Anzahl von Defekten zu beobachten. Die Kombination aus mehreren Hell- und Dunkelfeldabbildungen in unterschiedlichen Beugungsbedingungen zeigt, dass es sich bei den Defekten um volle Versetzungen handelt. Eine Auswahl an Dunkelfeldbildern mit den zugehörigen Abbildevektoren sind in Abbildung 5.39 zusammengestellt. Deutlich erkennbar ist auf diesen Bildern, dass mit den Defekten Dehnungskontraste verknüpft sind. Der relativ geradlinige Verlauf der Kontraste bildet charakteristische Winkel mit 


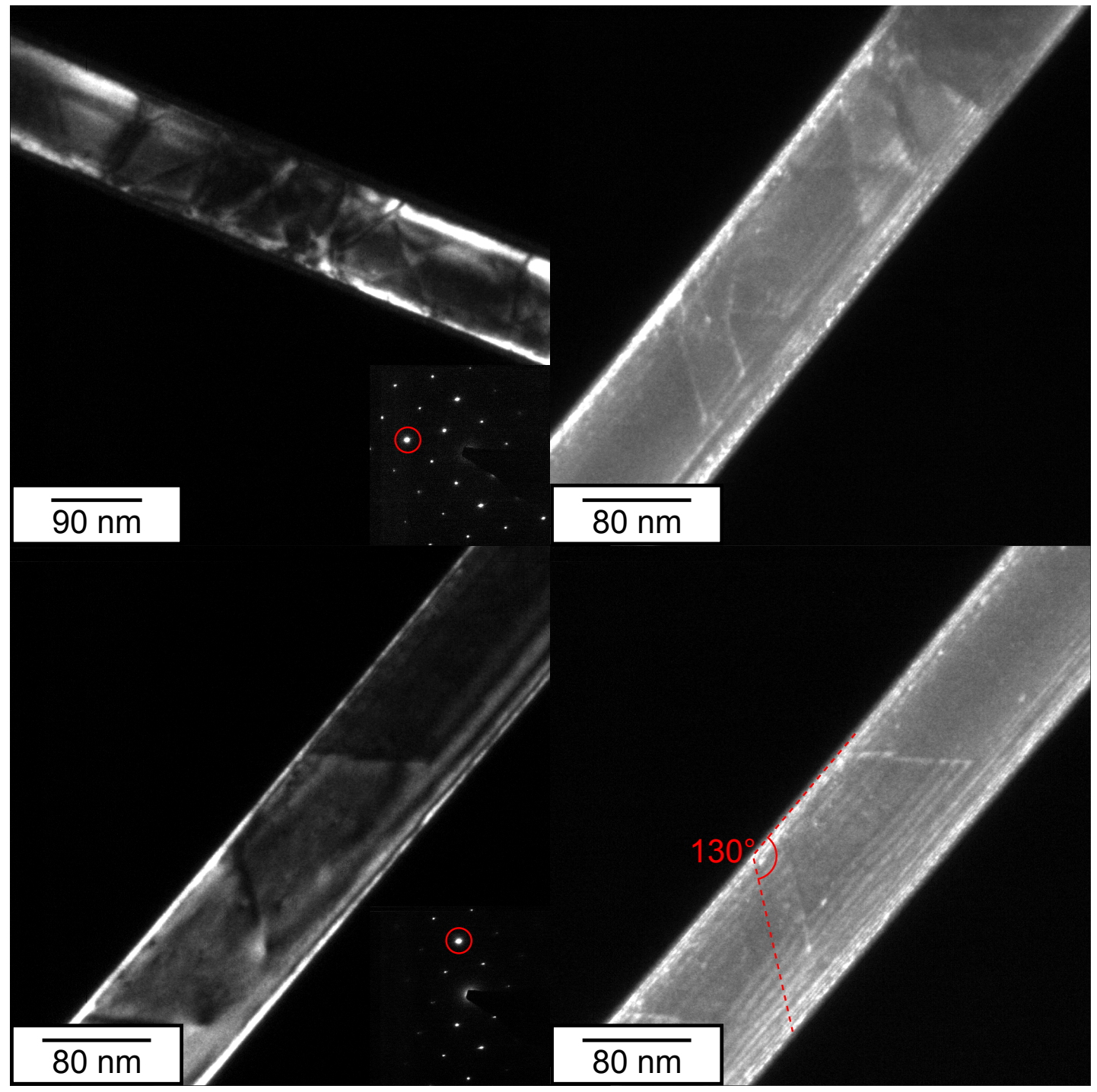

Abbildung 5.39: Versetzungen in einem verbogenen $110 \mathrm{~nm}$ Draht - Dunkelfeldbilder (links) und weak-beam Dunkelfeldbilder (rechts) von der Position im Draht, an der die Verformung stattgefunden hat. Die verwendeten Reflexe sind im Beugungsbild dargestellt. 
der Drahtachse. Misst man jeweils den größeren der beiden auftretenden Wechselwinkel erhält man einen Wert zwischen $125^{\circ}$ und $130^{\circ}$. Anhand der Beugungsbilder und der Kenntnis aus der Orientierung des Drahtes relativ zum Elektronenstrahl kann man aus den Winkeln Rückschlüsse auf die Eigenschaften der Defekte ziehen. Legt man die Drahtachse entlang der [110]-Richtung ist laut Tabelle 2.1 für eine Zugbelastung die Versetzung auf der (111)-Ebene mit einem Burgersvektor von [011̄] favorisiert. Das Linienelement liegt im Falle einer Stufenversetzung entlang der [211] Richtung. Diese schließt einen Winkel von $107^{\circ}$ mit der Zugachse ein. In der Projektion auf die [1]10] Richtung des Elektronenstrahls beträgt der somit sichtbare Winkel 125,3․ Dieser Wert stimmt sehr gut mit den gemessenen Winkeln überein.

Eine direkte quantitative Analyse der Burgersvektoren ist in dieser Probe nicht möglich, da durch die Verbiegung keine aussagekräftigen Zweistrahlfälle eingestellt werden konnten.

Im dritten Versuch wurde ein $260 \mathrm{~nm}$ Draht getestet. Hier war die Besonderheit, dass der Draht trotz seiner großen Breite noch im TEM transparent war. Die Belastung wurde wiederum so lange durchgeführt, $d$. h. der Draht wurde soweit verbogen, bis Kontraständerungen sichtbar wurden, die auf eine plastische Verformung hindeuten. Bei diesem Verbiegen wird im Video deutlich, dass sich die scheinbare Breite des Drahtes mit der Verformung verkleinert. Dies ist ein weiteres Indiz für die Vermutung, dass der Draht deutlich dünner ist als breit. Weiterhin kann man aus dieser Beobachtung schließen, dass die Verbiegung mit einer Verdrillung des Drahtes einhergeht und damit ein äußerst komplexer Spannungszustand vorliegen muss.

Die plastische Verformung erfolgte in diesem Fall wieder sehr lokal zentral in der Mitte zwischen Befestigung und STM-Spitze durch Abknicken. Ein Vorher-Nachher-Bild ist in Abbildung 5.40 gezeigt. Bei diesem Draht konnten einige TEM Dunkelfeldaufnahmen von der verformten Stelle gemacht werden, die die Defektstruktur zeigen. In diesen Aufnahmen zeigt sich, dass in dem $260 \mathrm{~nm}$ Draht deutlich mehr Defekte gespeichert wurden als bei den anderen beiden Drähten. In diesem Fall liegen an der Knickstelle Versetzungen vor, allerdings ist die Struktur der Defekte komplexer als die zuvor beobachteten geraden Versetzungslinien. Man kann erkennen, dass viele Versetzungsringe und Halbringe im Draht liegen.Man kann den Eindruck gewinnen, dass bestimmte Orientierungen der Linien vermehrt auftreten. Einige Beispiele der Defektstruktur sind in Abbildung 5.41 gezeigt. In einigen Fällen kann beobachtet werden, wie Versetzungs- 


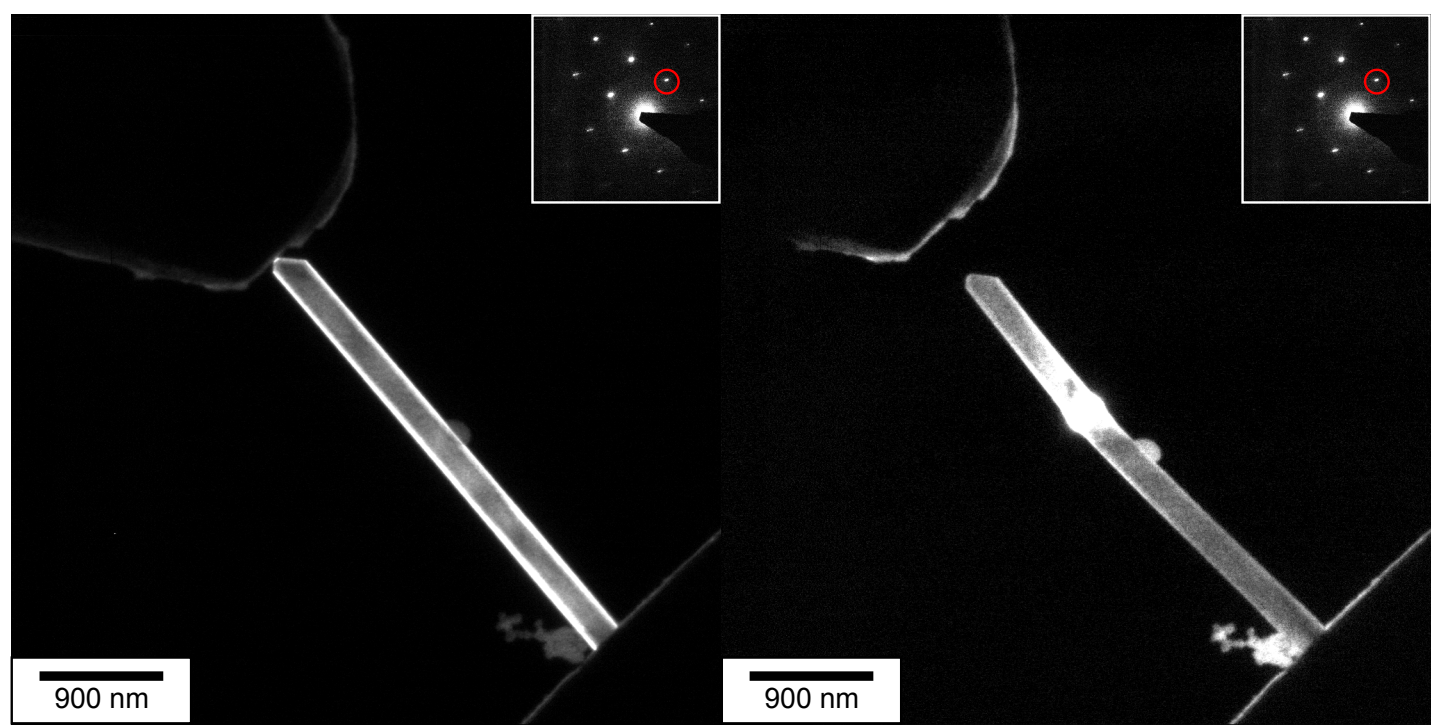

Abbildung 5.40: Dritter Biegeversuch - Dunkelfeldaufnahmen des $260 \mathrm{~nm}$ Drahtes vor (links) und nach der Verformung (rechts). Unter dieser Abbildebedingung erkennt man deutlich wo die lokale Verformung stattgefunden hat an dem hellen Bereich im rechten Bild.

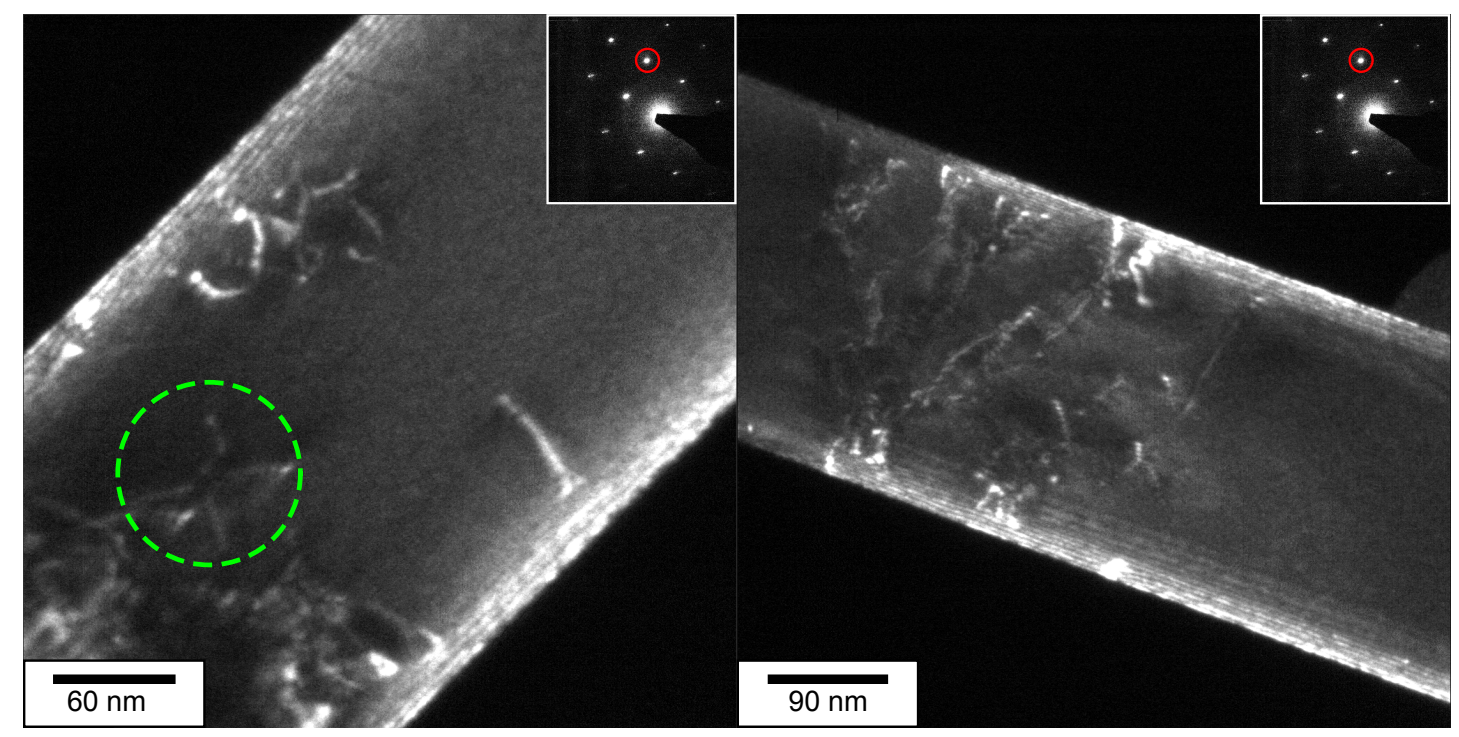

Abbildung 5.41: Defekte beim dritten Biegeversuch - TEM Dunkelfeldaufnahmen zeigen die Defektstruktur im $260 \mathrm{~nm}$ Draht nach erfolgter plastischer Verformung. Im linken Teilbild sind zwei wahrscheinlich an der Oberfläche beginnende Halbringe zu erkennen. 
linien vermutlich den Draht an der Oberfläche durchstoßen. In Abbildung 5.41 ist im linken Teilbild ein Beispiel gegeben, wo zwei gegenüberliegende Halbringe zu erkennen sind. Die symmetrische Lage lässt sich mit den beiden \{111\}-Ebenen erklären, auf denen sich die Versetzungen bewegen können. Legt man die Drahtachse entlang

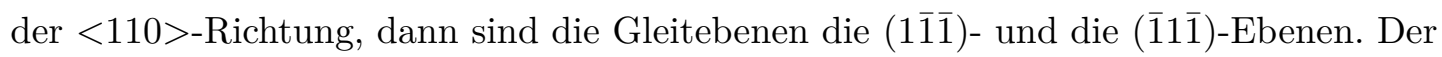
Abbildevektor aus Abbildung 5.41 ist $\mathrm{g}=(110)$. Damit ist jede Versetzung die sich auf diesen Ebenen bewegt, nach dem g.b Kriterium (vgl. Gleichung 2.31 in Kapitel 2.2) im TEM sichtbar. Der Burgersvektor der Versetzung ist folglich nicht weiter bestimmbar. Zusammenfassend kann man schließen, dass in allen getesteten Fällen aller Wahrscheinlichkeit nach Versetzungen die Verformung getragen haben. Dies gilt ebenfalls für den ersten Versuch, wo zwar keine Versetzungen direkt abgebildet werden konnten, aber auch keine Anzeichen für planare Defekte, wie sie durch Partialversetzungen hervorgerufen werden können, vorhanden waren. Der Bereich, auf dem die Drähte Verformung gezeigt haben, ist jeweils auf eine Knickstelle beschränkt geblieben. Die erreichten Spannungen für eine Versetzungsnukleation sind mit 3,9 GPa sehr hoch. 


\subsection{Vergleich der Verformungsexperimente}

In den vorangegangen Ausführungen wurden die Ergebnisse von Drähten mit unterschiedlichen Größen und Formen in verschiedenen Spannungszuständen aufgezeigt. Da aus der Literatur viele Beispiele bekannt sind, wo diese Eigenschaften eine wesentliche Rolle bei den Verformungsmechanismen spielen, sollen in diesem Abschnitt zusammenfassend die Einzelaspekte vergleichend dargestellt werden. Dabei wird jeweils das beobachtete Verformungsverhalten mit einer Eigenschaft des Drahtes verglichen, um daraus die für die Verformung wichtige Faktoren ableiten zu können.

\subsubsection{Drahtgröße und Defekttyp}

Der erste betrachtete Aspekt ist die Größenabhängigkeit des auftretenden Defekttyps im Zugversuch. Bisher wurden bei den Drähten als Größe jeweils die Breite, mit der der Draht im TEM erscheint, verwendet. Am Anfang wurde bereits erwähnt (vgl. dazu Kapitel 3.1), dass die Drähte durch \{111\}-Facetten an ihrer Oberfläche begrenzt werden. Die Länge dieser Facetten lässt sich in einigen Fällen aus den TEM Bildern vermessen. In Abbildung 5.42 sind an den Achsen die beiden Länge der Hauptfacetten aufgetragen. Die unterschiedlichen Symbole geben dabei an, welcher Defekttyp während der Verformung beobachtet werden konnte. Nicht von allen Drähten konnte im TEM allerdings die Facettenlänge bestimmt werden, sodass in diesen Fällen eine Unsicherheit hinsichtlich der Abmessungen bestehen bleibt. Daher wurde der Draht hinsichtlich seiner erscheinenden Breite eingeordnet und die Dicke des Drahtes über die Transparenz im TEM grob abgeschätzt. In der Grafik deuten die Fehlerbalken den anzunehmenden Bereich für die Dicke an.

Insgesamt konnten 19 Drähte in diese Grafik eingeordnet werden. Volle schwarze Symbole stellen dabei Drähte dar, die während der Verformung die in Kapitel 5.1 analysierten Stapelfehler und Zwillinge gezeigt haben. Dieses Verformungsverhalten tritt in einem Größenbereich von 35 bis 300 nm der längeren der beiden Facetten auf. Für die kürzere Facette wird der Bereich von 22 bis $160 \mathrm{~nm}$ abgedeckt. Die offenen Symbole geben die Dimensionen der Drähte wieder, die volle Versetzungen während der Verformung gezeigt haben (vgl. Kapitel 5.2). Wie dort schon erwähnt, wird diese Art von Defekt nur in Drähten beobachtet, bei denen die lange Facette größer als $150 \mathrm{~nm}$ ist. Für die kurze Facette hingegen beträgt der abgedeckte Größenbereich 20 bis $200 \mathrm{~nm}$ 
und ist somit noch größer als für die Stapelfehler.

Mit Hilfe dieser Aufstellung lassen sich einige Fragen beantworten. Zum einen wird

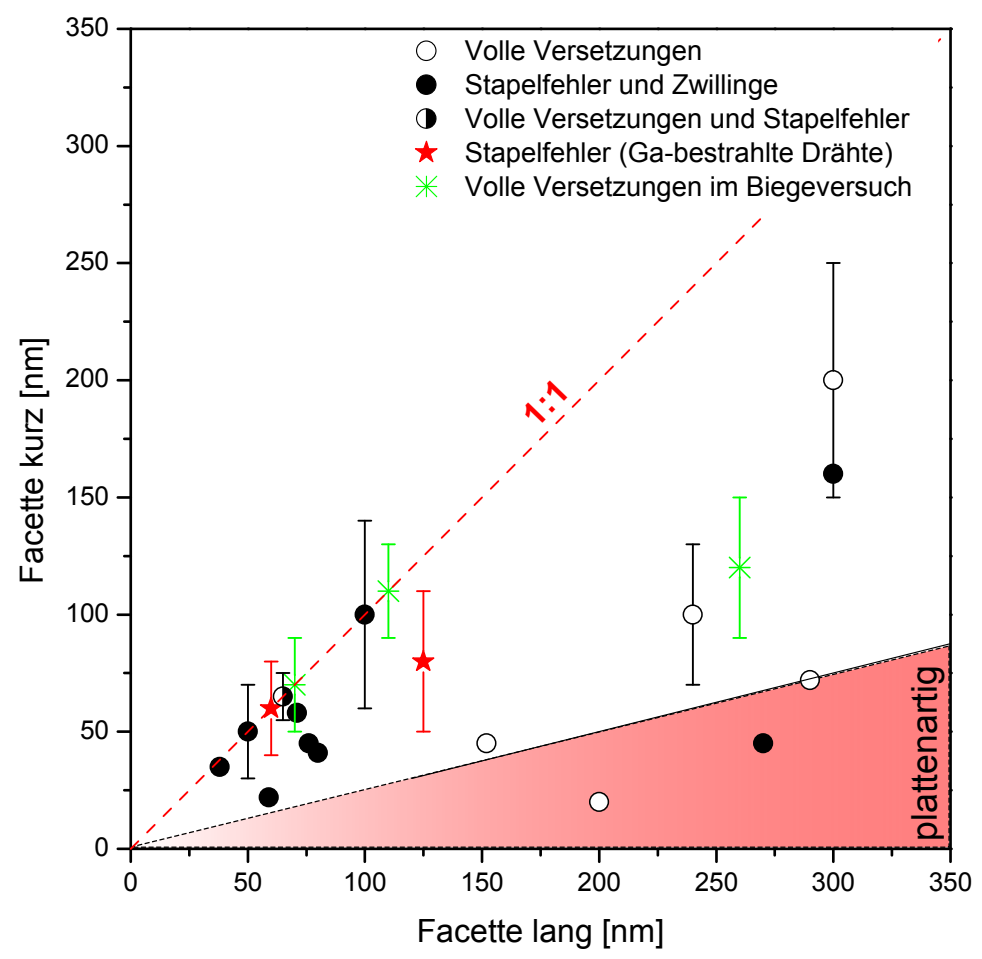

Abbildung 5.42: Größenabhängigkeit der Defekttypen - In diese Grafik sind die Defekttypen getesteter Drähte in Abhängigkeit von ihrer Facettelänge dargestellt. Schwarze Symbole beziehen sich auf Zugversuche. In Fällen in denen die die Facettenlänge nicht direkt ausgemessen werden konnte ist die erscheinende Breit aufgetragen und die lange Facette mit einer Unsicherheit dargestellt. Rote Symbole geben die Abmessungen der mit Ga-Ionen beschädigten Drähte wider. In grün sind die Biegeversuche dargestellt

deutlich, dass es für eine Zugbelastung in dem untersuchten Größenbereich keine obere Grenze gibt, bis wohin Partialversetzungen in den Drähten die Verformung tragen. Für volle Versetzungen lässt die Aufstellung allerdings den Schluss zu, dass es eine Mindestgröße gibt, ab der diese auftreten können. Diese Mindestgröße beträgt etwa $150 \mathrm{~nm}$ für die lange Facette.

Zum anderen kann die Frage untersucht werden, ob das Verhältnis der Facetten für den 


\section{ERGEBNISSE}

auftretenden Versetzungstyp ausschlaggebend ist . Dazu ist in Grafik 5.42 der Bereich rot hinterlegt, bei dem das Aspektverhältnis der Drähte größer ist als 4:1 und damit von einer plattenartigen Struktur gesprochen werden kann. Hier erkennt man, dass in diesem Bereich beide Arten von Defekten beobachtet werden konnten. Demzufolge ist die Form als der bestimmende Faktor für den Defekttyp auszuschließen.

\subsection{2 Überstruktur und Defekttyp}

Im Abschnitt über die Probencharakterisierung wurde darauf hingewiesen, dass in einigen Proben Extrapunkte im Beugungsbild auftraten. Für dieses Auftreten werden in der Literatur einige mögliche Erklärung geliefert, die jedoch nicht eindeutig den in dieser Arbeit untersuchten Proben zugeordnet werden konnten. Unabhängig davon, welcher der erwähnten möglichen Defekte für die in dieser Arbeit verwendeten Drähte zutrifft, muss untersucht werden, ob die mit der Überstruktur verbundene Mikrostruktur von Relevanz für die Verformung sein kann. Eine erste Abschätzung ist realtiv einfach möglich, indem man die während der Verformung auftretenden Defekttypen in Proben mit und ohne Extrapunke vergleicht. Dieser Sachverhalt ist zusammenfassend in Tabelle 5.4 dargestellt. Der Vergleich zeigt, dass sowohl volle Versetzungen als auch Stapelfehler in dem in dieser Arbeit abgedeckten Größenbereich in den Proben als charakteristische Defekte während der Verformung auftreten. Auffallend bleibt dabei jedoch, dass die Beobachtung von vollen Versetzungen bis auf einen Fall mit dem Auftreten der Überstruktur verknüpft ist. Das besondere Verformungsverhalten des $300 \mathrm{~nm}$ Drahtes, der als einziger keine Extrapunkte aufwies, wich allerdings generell von dem überlicherweise beobachteten homogenen Auftreten von Defekten ab. Wie in Abschnitt 5.2 gezeigt wurde, ist die Verformung von einer sehr lokalen Einschnürung geprägt. Im Falle eines $65 \mathrm{~nm}$ Drahtes konnten sowohl Versetzungen als auch Stapelfehler beobachtet werden, auch dieser Draht wies die Überstruktur in der $\{111\}$-Zonenachse auf. Abbildung 5.43 zeigt ein Dunkelfeldbild dieses Drahtes mit der besonderen Defektstruktur.

Zusammenfassend kann man folgern, dass das Auftreten der Überstruktur eindeutig mit einem Wechsel im Verformungsmechanismus verbunden ist. Da die Extrapunkte ein Indiz dafür sind, dass der Kristall von der Perfektion eines Einkristalls abweicht, ist davon auszugehen, dass die Mikrostruktur einen Einfluss auf den beobachteten Versetzungstyp hat. Unter Punkt 6 wird daher ein eigener Abschnitt die möglichen Wechselwirkungen 


\begin{tabular}{cccccc}
$\begin{array}{c}\text { Breite } \\
{[\mathrm{nm}]}\end{array}$ & Defekttyp & $\begin{array}{c}\text { Spannungs } \\
\text { zustand }\end{array}$ & Zonenachse & Überstruktur & Anzahl \\
\hline \hline$>190$ & Versetzung & Zug & $\{111\}$ & ja & 4 \\
300 & Versetzung & Zug & $\{111\}$ & nein & 1 \\
65 & Stapelfehler/ & Zug & $\{111\}$ & ja & 1 \\
& Versetzungen & & & & \\
110 & Stapelfehler & Zug & $\{111\}$ & nein & 2 \\
240 & Stapelfehler & Zug & $\{111\}$ & nein & 1 \\
110 & Versetzung & Druck & $\{111\}$ & ja & 1 \\
26 & Versetzung & Druck & $\{111\}$ & nein & 1 \\
\hline \hline
\end{tabular}

Tabelle 5.4: Einfluss der Überstruktur auf die Verformung - Diese Auflistung gibt an, in wie vielen Fällen eine Überstruktur im Beugungsbild identifiziert werden konnte. Dazu ist angegeben in welchem Belastunggszustand welcher Defekttyp beobachtet werden konnte.

von Versetzungen mit bekannten Defekten, wie Zwillingsgrenzen und Stapelfehlern, diskutieren.

\subsubsection{Zwillingsbildung und Defekttyp}

Eine weitere Eigenschaft der Form der Drähte ist in der Darstellung von Abbildung 5.42 nicht enthalten: das Auftreten von Nebenfacetten zusätzlich zu den $\{111\}$-Ebenen, die die Drähte sonst definieren. Diese so genannte "truncation" bezeichnet dabei das Abschneiden von im spitzen Winkel aufeinander treffenden Seitenflächen durch eine weitere kristallographisch niedrig indizierte Ebene. Durch eine Wulff-Konstruktion lässt sich zeigen, dass dort eine weitere Oberfläche gebildete wird, die durch eine $\{100\}$-Ebene begrenzt wird.

Einen entscheidenden Einfluss kann diese Art der Facettenbildung auf das Auftreten von Zwillingen als Resultat von korrelierter Bewegung von Partialversetzung haben. Hinweise darauf gibt es in der Literatur, wo die einfache Überführung von $\{111\}$-Oberflächen in energetisch niedrige $\{100\}$-Oberflächen durch das Vorhandensein der Nebenfacetten unterbunden wird [18, 25].

Eine abgeschnittene, scharfe Kante ist im REM nicht einfach abzubilden, da sie häufig nur in einem Bereich von $10 \mathrm{~nm}$ Größe auftreten. Einfacher als sich also den Querschnitt 


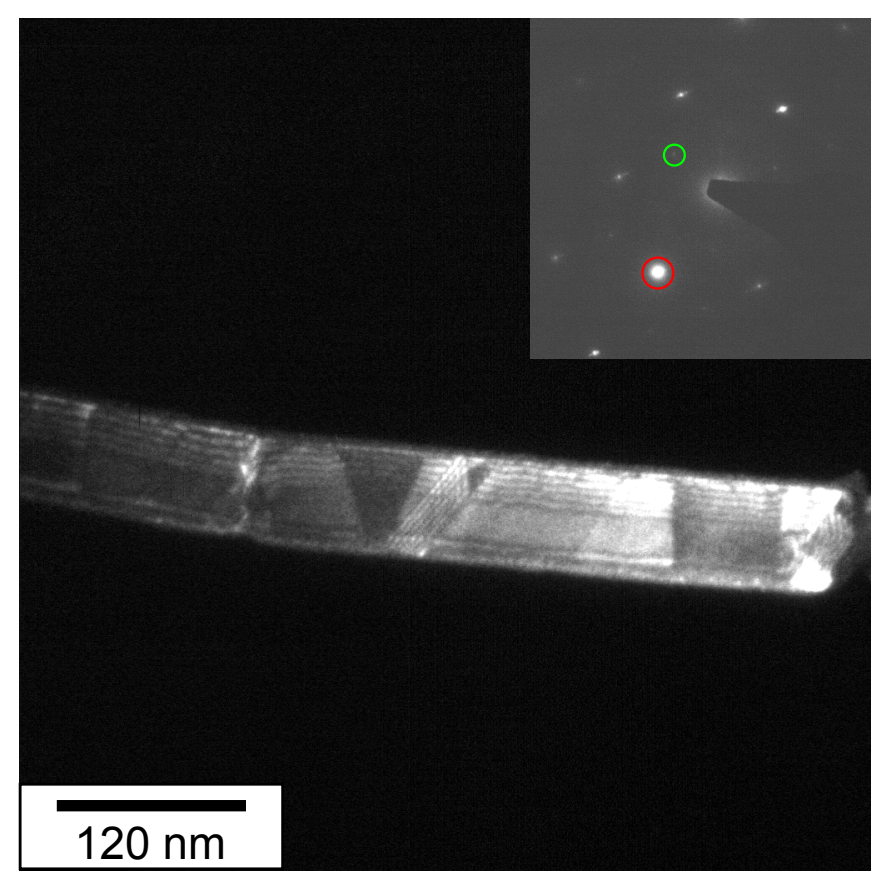

Abbildung 5.43: Verformter 65 nm Draht - Dunkelfeldaufnahme eines 65 nm Drahtes der nach der Verformung sowohl den oszillierenden Kontrast eines Stapelfehlers zeigt als auch in der linken Bildhälfte den Dehnungskontrast einer vollen Versetzung zeigt. Im Beugungsbild ist mit dem grünen Kreis die Lage der Überstrukturreflexe gekennzeichnet. Der rote Kreis markiert die Lage der $\{220\}$-Reflexe.

im REM anzuschauen, ist es, diese kurzen Facetten direkt anhand eines Stapelfehlers im TEM zu identifizieren. Dieser repräsentiert nämlich den Querschnitt des getesteten Drahtes auf der $\{111\}$-Ebene, auf der die Partialversetzung den Draht durchlaufen hat (vgl. dazu auch Abbildung 5.9). Unter günstigen Orientierungen ist es dann möglich diese Kristalleigenschaften zu untersuchen. Tabelle 5.5 zeigt eine Aufstellung von Drähten, in denen Zwillinge beobachtet wurden in Abhängigkeit davon, ob eine Nebenfacette vorlag. Man erkennt, dass die Mehrzahl der Drähte diese Nebenfacetten nicht aufwies. Dort wurden in allen Fällen Zwillinge beobachtet. Lag eine truncation vor, konnte auch jeweils immer eine Zwillingsbildung beobachtet werden. Damit ist mit relativer Sicherheit auszuschließen, dass das Vorhandensein einer Nebenfacette die Zwillingsbildung komplett unterdrücken kann. Ob allerdings ein Einfluss auf die Gesamtzahl oder die maximale Dicke der Zwillingslamellen besteht, konnte nicht geklärt werden. 


\begin{tabular}{cc} 
Form & $\begin{array}{c}\text { Zwillingsbildung } \\
{[\text { Anzahl/Gesamtzahl }]}\end{array}$ \\
\hline \hline truncated & $4 / 4$ \\
non-truncated & $>10 / 10$ \\
\hline \hline
\end{tabular}

Tabelle 5.5: Verzwillingung im Draht - Diese Auflistung gibt an, in wie vielen Fällen Zwillinge im Draht identifiziert werden konnten in Abhängigkeit vom Auftreten einer Nebenfacette.

\begin{tabular}{ccc} 
Belastungart & Defekttyp & Häufigkeit \\
\hline \hline Zugversuch & Stapelfehler & 21 \\
& Versetzung & 5 \\
Biegeversuch & Stapelfehler & 0 \\
& Versetzung & 3 \\
\hline \hline
\end{tabular}

Tabelle 5.6: Defekttypen nach Belastungsart - Diese Tabelle fasst zusammen, bei welcher Belastungsgeometrie welcher Defekttyp beobachtet wurde.

\subsubsection{Spannungszustand und Defekttyp}

In den vorangegangenen Abschnitten dieses Kapitels wurden zwei unterschiedliche Geometrien zum Testen der Nanodrähte vorgestellt. Einerseits wurden uniaxiale Zugversuche durchgeführt, andererseits Biegeversuche. Bei Letzteren lag durch die komplizierte Verformungsgeometrie ein triaxialer Spannungszustand vor. Dieser Umstand erlaubt es nun, den Einfluss des Spannungszustandes auf die Defektbildung zu betrachten.

Die Ergebnisse dieser Experimente sind in Tabelle 5.6 zusammengefasst. Dort wird der beobachtete Defekttyp in Abhängigkeit zu der gewählten Belastungsgeometrie dargestellt. Man erkennt, dass in Zugversuchen zwei Arten von Defekten beobachtet wurden: Stapelfehler und Zwillinge als wahrscheinliche Folge von Partialversetzungen, die den Draht innerhalb kürzester Zeit durchlaufen, und volle Versetzungen. Das unterschiedliche Auftreten beider Defekttypen wurde im vorangegangen Abschnitt eingehend erläutert. In Biegeversuchen hingegen wurden in allen Fällen volle Versetzungen beobachtet. Dieses Ergebnis lässt sich dahingehend so deuten, dass ein komplexer Spannungszustand dazu führt, dass keine isolierten Partialversetzungen den Draht durchlaufen können. Dies führt dazu, dass auch keine Stapelfehler beobachtet werden konnten. Allerdings muss man beachten, dass lediglich drei entsprechende Versuche durchgeführt wurden, 
bei denen nur zwei Drähte in einem Größenbereich lagen, in dem Stapelfehler erwartet wurden. 


\section{6}

\section{Diskussion}

In diesem letzten großen Kapitel der Arbeit sollen die Ergebnisse aus Abschnitt 5 in einem größeren physikalischen Zusammenhang diskutiert werden. Durch die experimentellen Untersuchungen wurden Fragen aufgeworfen, die einer eingehenderen Betrachtung bedürfen. Nimmt man die Zusammenfassung am Ende des vorherigen Kapitels als Grundlage für diese Betrachtung ergeben sich folgende zentrale Fragestellungen:

- Warum wird die Zugverformung besonders in Drähten mit $\mathrm{d}<180 \mathrm{~nm}$ durch die Bildung von Partialversetzungen getragen?

- Ist das Auftreten von vollen Versetzungen ein Größeneffekt oder kann dies allein durch die Mikrostruktur der Proben erklärt werden?

- Wodurch entscheidet sich, ob ein isolierter Stapelfehler gebildet wird, oder ein Zwilling während der Verformung entsteht?

- Warum befinden sich an den Bruchkanten der verformten Drähte häufig Zwillinge?

- Wodurch wird die räumliche Verteilung der Stapelfehler entlang der Drahtachse bestimmt?

- Warum hat der gewählte Spannungszustand einen Einfluss auf den Verformungsmechanismus eines Nanodrahtes?

Auf den folgenden Seiten soll die Klärung dieser Fragen angegangen werden. Dabei werden unter Zuhilfenahme der aktuellen Literatur die gewonnenen Ergebnisse eingeordnet und soweit es möglich ist im Rahmen schon bestehender Modelle erklärt. Da dies 


\section{DISKUSSION}

nicht für jeden Aspekt möglich ist, wird in einem weiteren Schritt die Beschreibung des Verformungsmodells weiterentwickelt werden. Diese Ergänzungen bringen dabei bisher existierende Konzepte in einer ganzheitlichen Beschreibung für die Versetzungsnukleation in Einklang.

\subsection{Zum Auftreten von Partialversetzung}

Die Beobachtungen aus Kapitel 5.1 zeigen deutlich, dass besonders in Drähten mit Abmessungen kleiner als einer kritischen Größe durchweg Partialversetzungen während der Verformung beobachtet werden können. In der Literatur wird das Auftreten von Partialversetzungen in sehr kleinen Proben als mögliche Konsequenz der spannungsabhängigen Aufspaltungsweite von Versetzungen während der Aktivierung erklärt [10]. Somit wäre der Verformungsmechanismus ein größenabhängiger Effekt. Inwieweit diese Herangehensweise in dem in dieser Arbeit untersuchten Fall die Ergebnisse erklären kann, soll daher vorweg in Abschnitt 6.1.1 genauer dargestellt werden.

Aus der Tatsache, dass vor der Verformung, wie in Abschnitt 3.2 gezeigt wurde, keine Versetzungen in dem Draht vorhanden sind, wird klar, dass plastische Dehnung nur möglich wird, wenn Versetzungen neu gebildet werden [57]. Für dieses Szenario ist es dafür von fundamentaler Bedeutung, den Aspekt der Nukleation von Versetzungen besser zu verstehen und zu beschreiben. Die Tatsache, dass es bis heute keine verlässliche Beschreibung für die Versetzungnukleation gibt, liegen darin begründet, dass es zur Zeit experimentell nicht möglich ist genauere atomistische Einblicke in diesen Vorgang zu erhalten. Die in diesen Prozess involvierten Größen- und Zeitskalen sind zurzeit nur sehr begrenzt zugänglich. Aus diesem Grund wurden in der Literatur unterschiedliche Betrachtungsweisen für die Nukleation entwickelt und die möglichen wichtigen Faktoren diskutiert. Zurzeit werden im Wesentlichen zwei grundverschiedene Konzepte zur Versetzungskeimbildung intensiver weiterentwickelt. Diese beiden Modelle werden in der Literatur unter den folgenden Terminologien zusammengefasst:

- Generalized Stacking Fault Energy - Die zu überwindende Energiebarriere für unterschiedliche Verformungswege ist definiert durch den Potentialverlauf, der sich durch starres Verschieben zweier Kristallhälften gegeneinander ergibt. Das Verhältnis von dabei auftretenden instabilen und stabilen Zuständen gibt Aufschluss über den zu erwartenden Defekttyp. 
- Classical Nucleation Theory - Versetzungskeimbildung wird als ein thermisch aktivierter Prozess behandelt. Im elastischen Modell werden kritische Keimradien berechnet, ab der ein Versetzungshalbring stabil ist. Die zugehörige Aktivierungsenergie gibt Auskunft über die Häufigkeit des jeweiligen Prozesses.

Bis jetzt ist nicht eindeutig geklärt, welches Modell für die Beschreibung geeigneter ist, da in allen Fällen Limitierungen auftreten, die nicht einfach in die Modelle integrierbar sind. In den folgenden Abschnitten soll nun genauer auf die einzelnen Modelle eingegangen werden. Dabei wird versucht, so weit wie möglich die experimentell zugänglichen Parameter in das jeweilige Modell einfließen zu lassen. Es wird aufgezeigt, ob die verwendeten Modelle in der Lage sind, die experimentellen Befunde zu erklären. Darüber hinaus werden physikalische Limitierungen der Betrachtungsweisen aufgezeigt und mögliche Konsequenzen für makroskopische Proben gezogen.

\subsubsection{Auspaltungsweite von Partialversetzungen}

In [31] wird gezeigt, dass bei angelegter äußerer Spannung der Abstand zwischen den beiden Partialversetzungen einer dissozierten Versetzung eine Funktion dieser Spannung wird. Die Idee des Ansatzes ist es daher, das isolierte Auftreten von Partialversetzungen damit zu erklären, dass mit einer hohen wirkenden Schubspannung die Aufspaltungsweite größer wird als die Probe. Man würde dann erwarten, dass eine einzelne Partialversetzung allein aufgrund der wirkenden Spannung die Probe komplett durchläuft und an der Oberfläche annihiliert wird. Für die Bildung der nachlaufenden Partialversetzung würde dann zu keinem Zeitpunkt eine Triebkraft existieren. In diesem Abschnitt soll nun untersucht werden, ob dieses Modell in der Lage ist, die korrekte Beschreibung für das beobachtete Verformungsverhalten und den korrekten Größenbereich zu liefern. Dazu werden die wesentlichen Gedanken aus [31] auf die Geometrie der in dieser Arbeit getesteten Drähte übertragen.

Für die Berechnung der allgemeinen Form der Aufspaltungsweite wird eine Geometrie betrachtet, wie sie in Abbildung 6.1 dargestellt ist. Die von außen angelegte Scherspannung zeigt in $\mathrm{x}$-Richtung. Damit ist die Aufspaltungsweite $d$ gegeben durch

$$
d=\frac{G b_{p}^{2} f\left(\theta_{1}, \theta_{2}\right)}{\pi\left(2 \gamma-\tau_{z x} b_{p}\left|\sin \left(\theta_{1}\right)-\sin \left(\theta_{2}\right)\right|\right)} .
$$




\section{DISKUSSION}

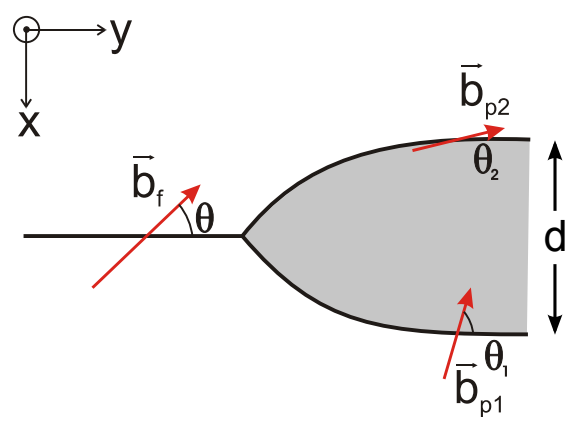

Abbildung 6.1: Zeichnung der Aufspaltungsgeometrie - Für die Berechnung der Aufspaltungsweite werden zwei Partialversetzugnen in der xy-Ebene und eine äußere Scherspannung in x-Richtung angenommen.

Die Größen $\theta_{i}$ bezeichnen hierbei die Winkel der Burgersvektoren zum zugehörigen Linienelement. Die Funktion $f(\theta)$ lautet

$$
f\left(\theta_{1}, \theta_{2}\right)=\cos \theta_{1} \cos \theta_{2}+\frac{\sin \theta_{1} \sin \theta_{2}}{1-\nu} .
$$

Diese Formulierung berücksichtigt dabei die Peach-Köhler Kraft, die auf die unterschiedlichen Versetzungssegmente wirkt, ebenso wie die elastische Wechselwirkung zwischen den beiden Partialversetzungen. Da bei der Aufspaltung ein Stapelfehler gebildet wird fließt auch dieser Energiebeitrag in die Abstandsformel mit ein. Mit der Gesamtheit dieser Beiträge lässt sich die Winkelabhängigkeit der Aufspaltungsweite grafisch darstellen. Dafür wurden typische Werte für Gold (Poissonzahl $\nu=0,44$ aus [58] und Schermodul $G=27$ GPa aus [10]) angenommen. Da eine feste Winkelbeziehung zwischen den Partialversetzungen und der zugehörigen vollen Versetzung besteht, ist es möglich, die Beschreibung über zwei Winkel auf einen Parameter zu reduzieren. Die Berechnungsvorschrift für den Winkel der vollen Versetzung lautet

$$
\begin{aligned}
& \theta_{2}-\theta_{1}=60^{\circ} \\
& \frac{\theta_{1}+\theta_{2}}{2}=\theta
\end{aligned}
$$

Gezeichnet ist in Abbildung 6.2 die Aufspaltungsweite für den Winkel zwischen Linienelement und zu einem Paar von Partialversetzungen gehöriger vollen Versetzung. Anhand dieser Auftragung wird deutlich, dass die Gleichgewichtsaufspaltung schon im spannungslosen Zustand eine Funktion des Winkels ist. Die Distanz variiert zwischen $0,8 \mathrm{~nm}$ für eine reine Schraubenversetzung $\left(\theta=0^{\circ}\right)$ und etwa $3 \mathrm{~nm}$ für eine Versetzung 


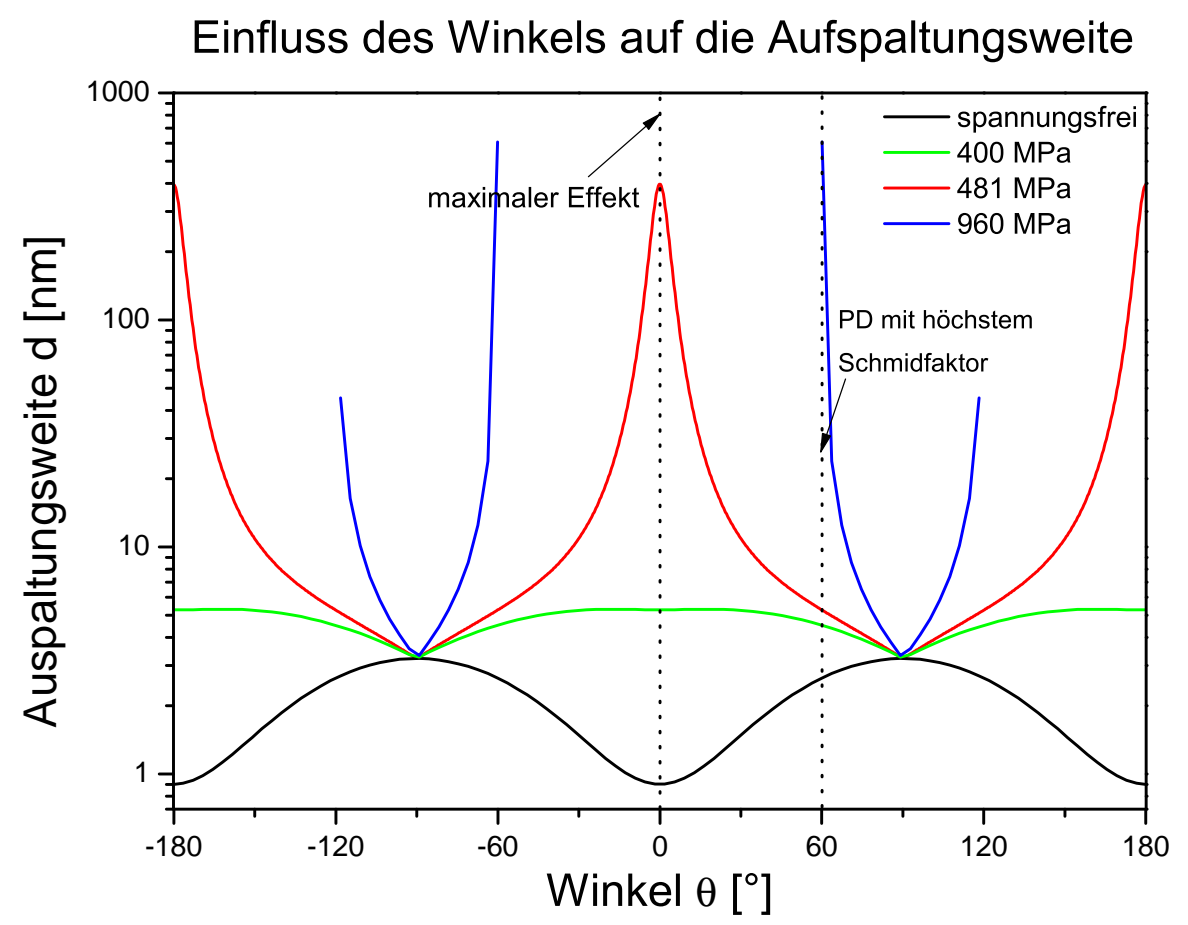

Abbildung 6.2: Winkelabhängigkeit der Aufspaltungsweite - Darstellung der Funktion 6.1 für verschiedene Werte der äußeren Scherspannung.

mit reinem Stufencharakter $\left(\theta=90^{\circ}\right)$. Diese Tatsache liegt in der Wechselwirkung des Spannungsfeldes der einzelnen Partialversetzungen begründet. Dieser ist für die Teilversetzungen stark unterschiedlich, da er abhängig von dem Winkel zum Linienelement ist.

Unter angelegter äußerer Scherspannung wird eine weitere Eigenschaft deutlich: Die Aufspaltungsweite ist demnach unabhängig von $\tau$ für reine Stufenversetzungen. Dies ist an den Fixpunkten in Abbildung 6.2 bei $\theta=90^{\circ} \mathrm{zu}$ erkennen. Der Unterschied liegt darin begründet, dass die wirkenden Kräfte durch die angelegte Spannung auf die individuellen Partialversetzungen für diesen Fall identisch sind. Der maximale Effekt wird für $\theta=0^{\circ}$ beobachtet. Die Aufspaltungsweite divergiert hier sogar für Spannungswerte größer als $481 \mathrm{MPa}$.

Projiziert man diese Tatsachen auf die in dieser Arbeit verwendete Geometrie, muss die Aufspaltungsweite für einen bestimmten Wert $\theta$ betrachtet werden. Folgt man nämlich der kristallographischen Beziehungen aus Abschnitt 2.7, wird ersichtlich, dass genau 


\section{DISKUSSION}

eine Partialversetzung pro Gleitebene den höchsten Schmidfaktor (vgl. dazu auch Tabelle 2.1) hat. Damit wird diese nukleiert und es muss für die beiden möglichen nachlaufenden Partialversetzungen die Aufspaltungsweite berechnet werden. Für diese Fälle beträgt der Winkel zwischen korrespondierender voller Versetzung $\pm 60^{\circ}$. Aus Abbildung 6.2 wird deutlich, dass der Effekt auf diese Versetzung bedeutend geringer ist als für den zuvor erwähnten Fall der reinen Schraubenversetzung. Stellt man den Verlauf der Aufspaltungsweite für diese Versetzung in Abhängigkeit von der angelegten äußeren Spannung dar, gelangt man zu Abbildung 6.3 Die Aufspaltungsweite ist für diesen

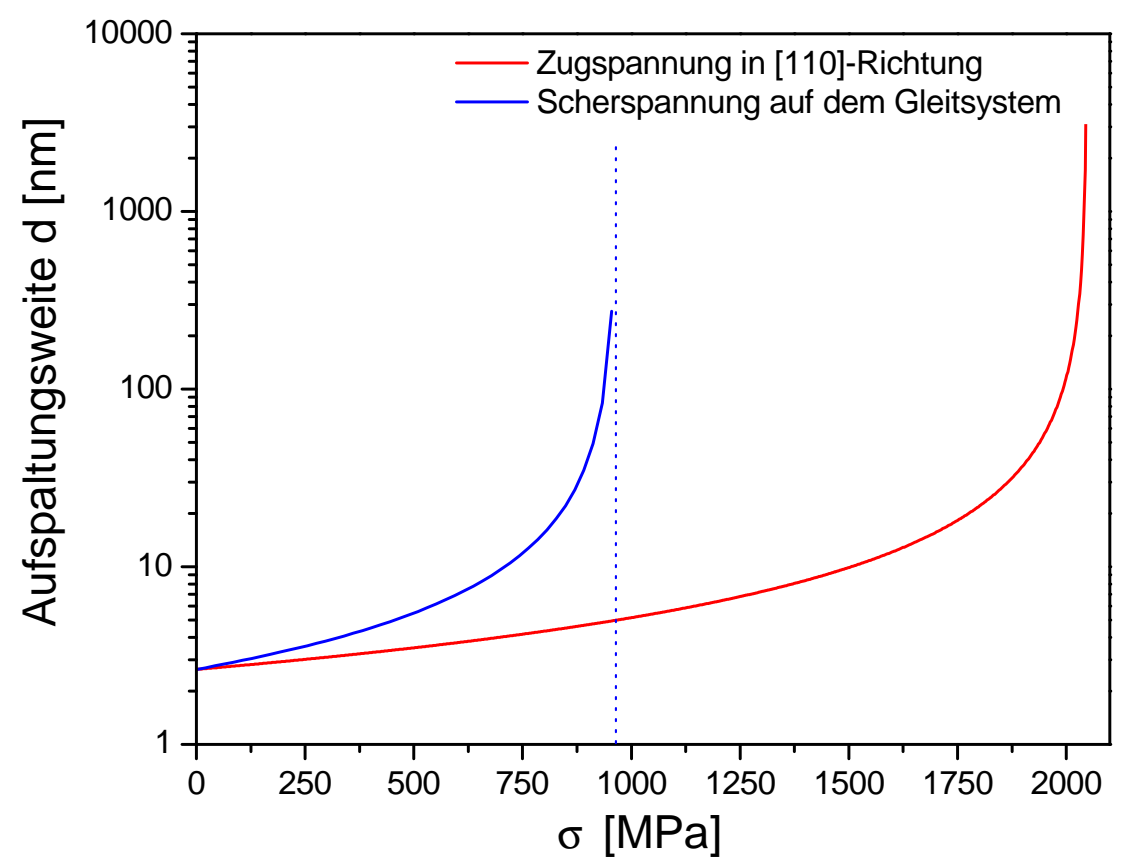

Abbildung 6.3: Verlauf der Aufspaltungsweite in Abhängigkeit einer angelegten Scherspannung - Der Verlauf der Aufspaltungsweite zeigt bei $\tau=0$ den Gleichgewichtsabstand der Versetzung mit $\theta=60^{\circ}$. Die Distanz divergiert für $\tau>980 \mathrm{MPa}$.

Spezialfall gegeben durch

$$
d(\tau)=\frac{G b_{p}^{2}}{2 \pi(1-\nu)\left(2 \gamma-\tau b_{p} \sin 30^{\circ}\right)} .
$$

Damit beträgt die Gleichgewichtsaufspaltung $\mathrm{d}_{0}$ bei $\tau=0$

$$
d_{0}=\frac{G b_{p}^{2}}{4 \pi \gamma(1-\nu)} .
$$


Die Aufspaltungsweite divergiert für Spannungen größer als $\tau_{\infty}$, welches die Polstelle in Abbildung 6.3 kennzeichnet. Der Wert hierfür lautet

$$
\tau_{\infty}=\frac{4 \gamma}{b_{p}} .
$$

Mit diesen Abkürzungen ist die Formel für den Abstand d reduzierbar auf eine Formel wie sie äquivalent auch in [59] verwendet wird:

$$
d(\tau)=\frac{d_{0}}{1-\tau / \tau_{\infty}}
$$

Für Gold lassen sich nun all diese materialspezifischen Größen berechnen. Aus quantitativen mechanischen Tests ist bekannt, dass die Nanodrähte typischerweise eine Fließspannung von etwa 1,2 GPa haben [24]. Mit dieser Größe und dem bekannten Schmidfaktor lässt sich die Scherspannung auf dem entsprechenden Gleitsystem berechnen. Diese beträgt nach Kapitel 2.1.2

$$
\tau=0,471 \cdot 1,2 \mathrm{GPa} \approx 565 \mathrm{MPa} .
$$

Da dieses Beispiel eine senkrecht zum Linienelement wirkende Scherspannung annimmt, ist der berechnete Wert für die Aufspaltungsweite als obere Grenze anzusehen. Bei einer wirkenden Scherspannung von $565 \mathrm{MPa}$ beträgt die Aufspaltungsweite demnach maximal ungefähr 6,4 nm. Der vorhergesagte Übergang, ab wann mit der Nukleation der nachlaufenden Versetzung zu rechnen ist, trifft somit überhaupt nicht den experimentell gefunden Größenbereich. Dort sind Partialversetzungen bis über 160 nm Probengröße beobachtet worden. Daher ist die spannungsabhängige Aufspaltungsweite allein nicht in der Lage das beobachtete Verhalten zu erklären. Diese liegt deutlich unter der Größe der Drähte, in denen die Partialversetzungen beobachtet wurden.

Versucht man zu ergründen, warum das Modell nicht genau den erwarteten Wert liefert, müssen die Schwächen der anfänglichen Annahmen betrachtet werden. Zum einen wird dort ein gerades Linienelement betrachtet, das in einer definierten Richtung einer Scherspannung ausgesetzt wird. Die Ableitung der skalaren Form in Gleichung 6.1 ist nur für diese Geometrie einfach möglich. Gekrümmte Linienelemente weisen einen zusätzlichen Term in der Beschreibung der Linienenergie auf, deren Beiträge in diesem Modell keine Betrachtung finden. Eine Erweiterung um diese Beiträge macht das Problem allerdings analytisch sehr viel komplexer und wird daher in diesem Rahmen nicht weiter diskutiert. Dies erscheint auch deswegen gerechtfertigt, weil keinerlei Hinweise 


\section{DISKUSSION}

auf eine Abhängigkeit der Versetzungsringform in unterschiedlich geformten Drähten beobachtet werden konnte. Zudem ist anzunehmen, dass die Erweiterung des Modells um diese Terme die Werte um einen Faktor zwei korrigiert.

Ein weiterer wichtiger Punkt bei der Betrachtung von Defekten in kleinskaligen Proben ist die Versetzungsnukleation. Da die verwendeten Drähte anfänglich frei von existierenden Defekten sind, wird klar, dass die Versetzungskeimbildung der kritische Schritt für die Verformung dieser Proben ist. Der Zusammenhang zwischen Versetzungskeimbildung und Aufspaltungsweite wird jedoch weder in der Darstellung von Byun et al. [31] noch in anderen Arbeiten [60, 52] berücksichtigt. Hier sind lediglich die verschiedenen wechselwirkenden Terme bei einer aufgespaltenen vollen Versetzung und damit zwei existierenden Partialversetzungen in Betracht gezogen worden. Die Ergebnisse dieser Arbeit legen jedoch nahe, dass während der Verformung nur eine Partialversetzung gebildet wird und diese sich zu jeder Zeit somit isoliert im Drahtvolumen aufhält. Damit ist auch eine Wechselwirkung zwischen zwei Versetzungen ausgeschlossen. Wenn dieses Bild Anwendung finden sollte, muss man die Aussagen über die Aufspaltungsweite also vielmehr als mögliche Triebkraft für die Nukleation einer nachlaufenden Partialversetzung verstehen.

In den folgenden Abschnitten soll nun versucht werden, ein tieferes Verständnis von der Versetzungskeimbildung und die damit verbundenen Defekttypen zu erlangen.

\subsubsection{Einführung in die Konzepte der Generalized Stacking Fault Energy}

Im vorangegangenen Abschnitt wurde ausführlich erläutert, wie das Auftreten von Stapelfehlern als Resultat der Aufspaltungsweite zwischen Partialversetzungen verstanden werden kann. Es wurde deutlich, dass die Vorhersage des Modells nicht den Größenbereich trifft, in dem in dieser Arbeit Stapelfehler beobachtet wurden. Es ist vorstellbar, dass die atomaren Prozesse, die sich während der Bildung einer Versetzung abspielen, einen wesentlichen Einfluss auf die Verformung haben. Daher muss ein anderer Ansatz gefunden werden die Ergebnisse schlüssig zu erklären. Dieser Ansatz beinhaltet neben dem geometrischen Argument auch die materialspezifische Energielandschaft, die während der kontinuierlichen Verformung zum Tragen kommt. Die Grundideen dieses Konzeptes sollen im Folgenden vorgestellt werden.

Nach [18] wird der Versetzungstyp, der während der Verformung beobachtet wird, 
durch eine Schmidfaktor-Analyse bestimmt. Dieses einfache Argument zur Vorhersage von Verformungsmechanismen ist folglich erst einmal ein rein geometrisches. Demnach wird immer dann eine isolierte Partialversetzung beobachtet, wenn in der vorliegenden Belastungsgeometrie der Schmidfaktor für die leading Partialversetzung größer ist als für die nachlaufende trailing Partialversetzung. Dies ist, wie in Tabelle 2.1 angegeben, auch die Situation bei einem Zugversuch in [110]-Richtung. Ist allerdings die aufgelöste Scherspannung für die nachlaufende Partialversetzung die größere, dann sagt das Modell die Verformung über volle Versetzungen voraus. Dies liegt darin begründet, dass die trailing Partialversetzung direkt zur leading Partialversetzung aufschließt. Damit sind keine isolierten Partialversetzungen möglich. Bei einer Belastungsrichtung in [110]-Richtung wäre dies zum Beispiel umsetzbar, wenn man vom Zugversuch in einen Druckversuch wechselt.

Die Übereinstimmung dieser einfachen Überlegung mit sowohl experimentell als auch in Simulationen beobachtetem Verformungsverhalten ist relativ groß [61]. Ausnahmen von dem vorhergesagten Verhalten finden sich in Geometrien, bei denen die Oberflächen der Drähte eine Besonderheit aufweisen. So wurde bei Kupfer-Nanodrähten mit $\{100\}$-Seitenfacetten beobachtet, dass unter Zugverformung eine Transformation der Oberflächen stattfindet. Durch die Nukleation von Partialversetzungen kommt es zur Umorientierung der Oberflächen zu \{111\}-Facetten [62].

An die Grenzen stößt das Modell bei der Möglichkeit der Vorhersage, ob isolierte Stapelfehler gebildet werden oder die Zwillingsbildung favorisiert ist. Dies liegt in der Einfachheit des Modells begründet. In dieser sind die Schmidfaktoren für die zwillingsbildende Partialversetzung und die leading Partialversetzung identisch und somit ist eine Unterscheidung zwischen den beiden Varianten nicht möglich. Darüber hinaus werden auch an Proben aus Aluminium Abweichungen von der Vorhersage festgestellt $[63,64]$. Gerade der Fall von Aluminium zeigt, dass bei Materialien mit einer hohen Stapelfehlerenergie die einfachen geometrischen Überlegungen versagen. Ein differenzierteres Bild, das auch materialspezifische Eigenschaften berücksichtigt, liefert hier das Konzept der generalisierten Stapelfehlerenergie (GSFE). Dieses soll im nächsten Abschnitt erläutert werden. 


\section{DISKUSSION}

\subsubsection{Generalized Stacking Fault Energy}

Neben der Möglichkeit den Stapelfehler als feststehende Größe zu interpretieren, die sich ergibt, wenn eine Partialversetzung durch einen ungestörten Kristall läuft hat Vitek ein differenzierteres Bild entwickelt. In seiner Betrachtungsweise ist die Stapelfehlerenergie vielmehr ein Wert, der ganz wesentlich von der aktuellen Größe des Burgersvektors abhängen muss [65]. Das verwendete Bild liefert den Energieverlauf für zwei gegeneinander starr verschobene Kristallhälften in Abhängigkeit von der Verschiebungsrichtung. Die Kurve für die Verschiebung in [112]-Richtung zeichnet sich dabei im Wesentlichen durch das Auftreten eines Minimums bei 1/6[112] aus. Dieses Minimum korrespondiert mit der Energie, die einem intrinsischen Stapelfehler zugeordnet werden kann. Der Energiewert $\gamma_{i S F}$ ist identisch zu dem, der für die klassische Nukleationstheorie verwendet wird. Auf dem Weg in diese Konfiguration muss allerdings erst ein Maximum überwunden werden. Dieses wird als instabiler Stapelfehler (engl. unstable stacking fault (USF)) mit der Energie $\gamma_{u S F}$ bezeichnet. Wie der Name andeutet, ist diese Konfiguration instabil und somit experimentell nicht direkt nachweisbar.

Bewegt man sich weiter in dieselbe [112]-Richtung, gelangt man zu einer Konfiguration, in der benachbarte Atome direkt übereinander im Atomgitter sitzen. Diese Lage ist als Position D in Abbildung 6.4 dargestellt. Mit ihr ist eine sehr hohe Energie verbunden. Dieses erste Bild der verallgemeinerten Stapelfehlerenergie lässt sich nun erweitern für den Fall der Nukleation sowohl einer leading als auch einer trailing Partialversetzung. Dies wurde durch Rice et. al. anhand der Versetzungsnukleation an einer Rissspitze durchgeführt [66]. In diesem Bild kombiniert man das errechnete Potential für die erste Partialversetzung (Bereich von A bis $\mathrm{C}$ in Abbildung 6.4) mit dem neu berechneten Verlauf für eine Verschiebung in Richtung der trailing Partialversetzung. Diese geht von einem Kristall aus, der bereits einen Stapelfehler beinhaltet und sich demnach auf dem Energieniveau von $\gamma_{i S F}$ befindet. Allerdings liegt nun durch den Richtungswechsel zwischen der ersten und zweiten Verschiebungsrichtung ein Winkel von $60^{\circ}$ vor, sodass nicht das sehr hohe Maximum auftritt. Vielmehr wird aus Abbildung 6.5 klar, dass ein Punkt überwunden werden muss, der eine äquivalente Koordination hat wie Position B in Abbildung 6.4. Somit ist auch die damit verbunden Stapelfehlerenergie wiederum $\gamma_{u S F}$.

Der Prozess der Zwillingsbildung bedarf nun einer weiteren Ergänzung des Bildes. Es ist 

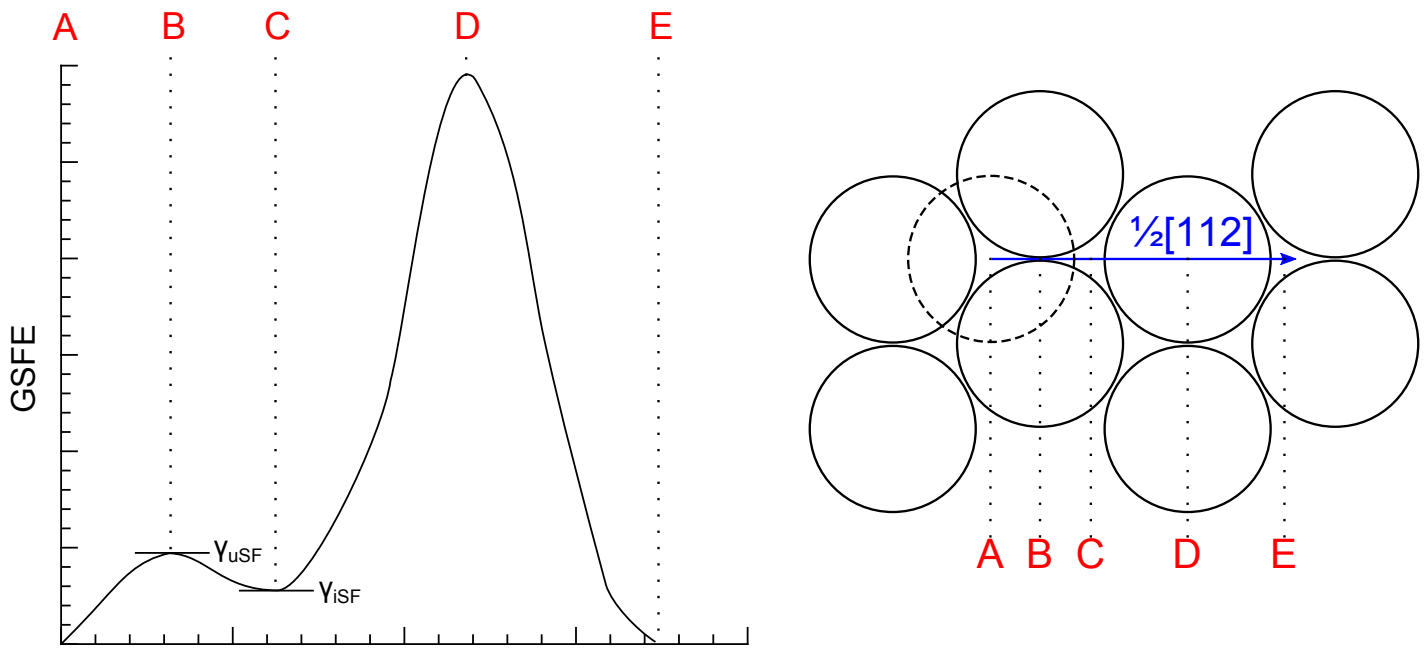

A B C D E

Verschiebung in [112]-Richtung

Abbildung 6.4: GSFE Kurve - Verlauf der GSFE für das starre Verschieben zweier Kristallhälften auf einer $\{112\}$-Ebene gegeneinander in [112]-Richtung. Im linken Bild ist der Verlauf der Energie schematisch für ein fcc Gitter aufgezeigt. Die mit verschiedenen Positionen verknüpfte Stapelfehlerenergie ist auf beiden Seiten markiert. Das Minimum in der Kurve wird mit der Energie $\gamma_{i S F}$ für einen intrinsischen Stapelfehler bezeichnet. Das zuvor liegende Maximum bezeichnet die Energie für einen instabilen Stapelfehler $\gamma_{i S F}$. Das sehr hohe Maximum auf der rechten Seite stellt die Situation dar, wenn zwei benachbarte Atome direkt aufeinander liegen. [65] 


\section{DISKUSSION}

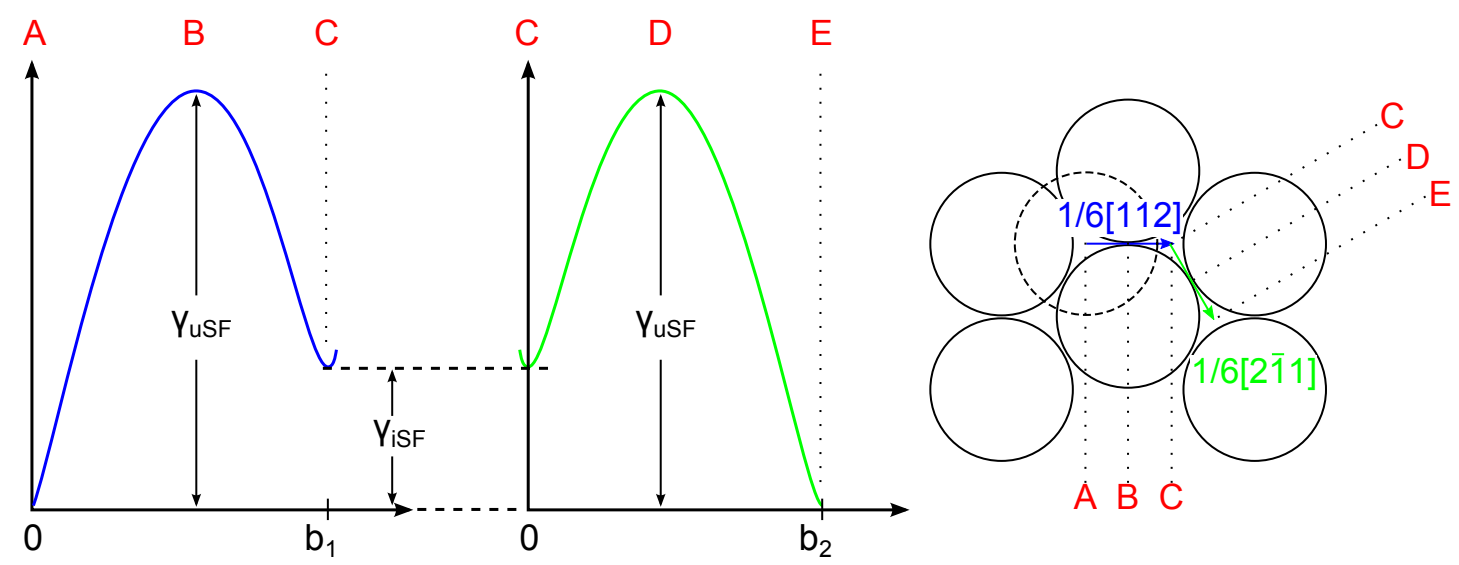

Abbildung 6.5: Potentialverlauf für die Bildung der Partialversetzungen mit unterschiedlichen Richtungen - Die leading Partialversetzung A muss die Energiebarriere von $\gamma_{u S F}$ überwinden um einen intrinsischen Stapelfehler zu bilden. Da das Gitter danach gestört ist, braucht die trailing Partialversetzung nur noch die Barriere der Höhe $\gamma_{u S F}-\gamma_{i S F}$ zu überwinden, um den Stapelfehler wieder auszulöschen (nach [66]).

klar, dass sich das Gesamtsystem nach dem Durchlaufen der ersten Partialversetzung auf dem Energieniveau $\gamma_{i S F}$ befindet. Eine zwillingsbildende Partialversetzung hat nun dieselbe Verschiebungsrichtung wie die erste, läuft allerdings auf einer benachbarten $\{111\}$-Eben durch den Kristall. Der mit dieser Verschiebung verknüpfte Energieverlauf ist in Abbildung 6.6 gezeigt. Das zu überwindende Maximum hat die Höhe $\gamma_{u T}$ (uT - unstable twinning). Der Zwillingsbildungsmechanismus ist abgeschlossen, wenn mit fortschreitender Verschiebung das Minimum auf Höhe von $2 \gamma_{T}{ }^{*}$ erreicht wird. Dieses ist fast die Energie von zwei voll ausgebildeten Zwillingsgrenzfläche $\gamma_{T}$, mit dem Unterschied, dass die Wechselwirkung der an der Grenzfläche beteiligten Atome berücksichtigt ist $[67,68]$. Ist der Zwilling groß kann diese vernachlässigt werden und $\gamma_{T}^{*}=\gamma_{T}$.

Wie schon in den theoretischen Grundlagen gezeigt, hat ein sich verformendes System nach erfolgter Nukleation der ersten Partialversetzung verschiedene Möglichkeiten der weitergehenden Verformung. Dabei hängt im Bild der GSFE der gewählte Kanal lediglich davon ab, wie das Verhältnis der beteiligten Stapelfehlerenergien ist. Man kann diese Tatsache zusammenfassen in der Beziehung für die Wahrscheinlichkeit der Zwillingsbildung T [69]

$$
T \propto \frac{\gamma_{u S F}-\gamma_{i S F}}{\gamma_{u t}-\gamma_{i S F}}
$$




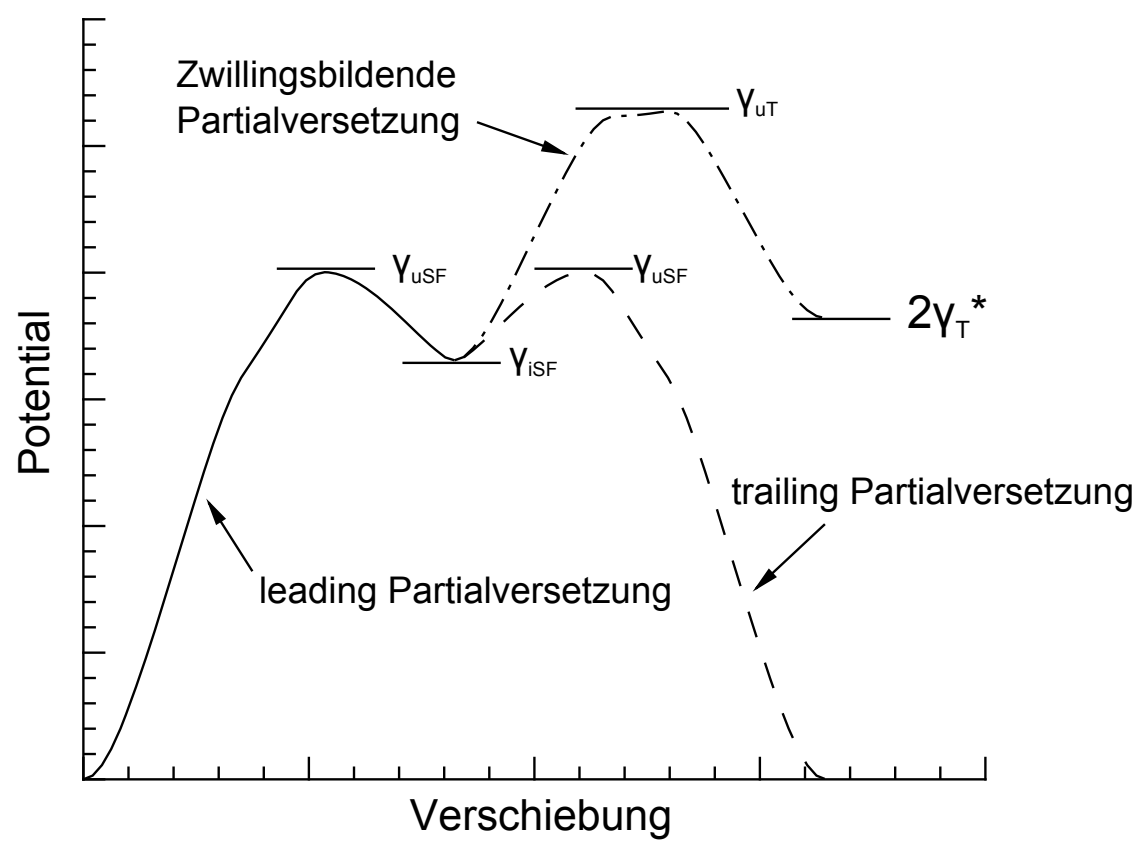

Abbildung 6.6: Potentialverlauf für die Zwillingsbildung - Für die Bildung eines Zwillings stellt sich die Potentiallandschaft des Kristall wie folgt dar: Die erste Partialversetzung folgt der durchgezogenen Linie und endet mit dem Stapelfehler auf $\gamma_{S F}$. Die zweite Partialversetzung auf einer benachbarten Ebene muss das Maximum mit Höhe von $\gamma_{U T}$ überwinden, um den embryonalen Zwilling zu bilden. (nach [67])

Dies besagt, dass die Wahrscheinlichkeit einer Zwillingsbildung dann hoch ist, wenn der Unterschied der Barrieren für den instabilen Stapelfehler und den instabilen Zwilling klein ist. Diese Argument ist demnach ein rein materialspezifisches.

Vereinigt man nun sowohl das geometrische Argument, das die aufgelösten Schubspannungen berücksichtigt, mit dem Bild des verallgemeinerten Stapelfehlers, erhält man ein erweitertes Konzept für das Vorhersagen von Verformungssmechanismen [61]. Dazu ist nur nötig, die unterschiedlichen Energiebarrieren mit den zugehörigen Schmidfaktoren zu gewichten. Für den Unterschied zwischen einer leading Partialversetzung $\left(\mathrm{S}_{L P}\right)$ und einer trailing Partialversetzung $\left(\mathrm{S}_{T P}\right)$ gilt

$$
\tau_{2}=\frac{\gamma_{u S F}-\gamma_{i S F}}{\gamma_{u t}-\gamma_{i S F}} \cdot \frac{S_{L P}}{S_{T P}}
$$

Damit entspricht der Vorfaktor dem in [70] eingeführten Parameter T. Der Unterschied zwischen der Bildung einer neuen isolierten Partialversetzung und einer zwillingsbil- 


\section{DISKUSSION}

denden Partialversetzung $\left(\mathrm{S}_{T W}\right)$ lässt sich darstellen als

$$
\tau_{1}=\frac{\gamma_{u S F}}{\gamma_{u t}-\gamma_{i S F}} \cdot \frac{S_{L P}}{S_{T W}}=\frac{\gamma_{u S F}}{\gamma_{u t}-\gamma_{i S F}} .
$$

Da für eine ideale Geometrie die Schmidfaktoren für die beiden Prozesse gleich sind vereinfacht sich in diesem fall die Darstellung für $\tau_{1}$.

\section{Einordnung der experimentellen Ergebnisse}

Mit Hilfe der vorher beschriebenen beiden Parameter können nun Ergebnisse sowohl material- als auch geometrieabhängig vorausgesagt werden. Für einen Zugversuch von Gold-Nanodrähten in [110]-Richtung ergibt sich eine Einordnung, wie es in Abbildung 6.7 gezeigt ist. Die Positionierung innerhalb des Diagramms wird im Wesentlichen davon bestimmt, welche Werte für die experimentell nicht zugänglichen Stapelfehlerenergien verwendet werden. Für Abbildung 6.7 kamen sowohl Werte aus tight-binding Rechnungen [30] zur Verwendung als auch Werte, die durch ein embedded atom Modell berechnet wurden [61]. Der Parameter $\tau_{1}$, der den Unterschied zwischen zwillingsbildender Partialversetzung und leading Partialversetzung angibt, liegt für diese Geometrie bei etwa 1,1, was nahe am Übergang zum Bereich von isolierten Partialversetzungen liegt. Daher gibt es mehrere mögliche Erklärungen, warum auch isolierte Partialversetzungen beobachtet werden. Auf der einen Seite zeigt sich, dass durch die Unsicherheit in den Werten für die instabilen Konfigurationen auch eine Ungenauigkeit bezüglich der Einordnung auf der $\tau_{1}$-Achse besteht. Auf der anderen Seite lässt sich eine Verschiebung herbeiführen, wenn man von lokalen Spannungskonzentrationen an den Nukleationspunkten auf der Drahtoberfläche ausgeht. Diese können dazu führen, dass die Schmidfaktoren zwischen den beiden konkurrierenden Prozessen lokal doch unterschiedlich sind und einen Wechsel in den Partialversetzungsbereich ermöglichen.

Eine weitere Verformungsgeometrie lässt sich zusätzlich in Abbildung 6.7 integrieren. Wie in Abschnitt 5.1 gezeigt wurde bilden sich während der Verformung auch Zwillingslamellen aus. Für diese Bereiche liegt ein Zugversuch in [114]-Richtung vor. Die leading Partialversetzung hat in dieser Orientierung einen Schmidfaktor von 0,419 und die trailing Partialversetzung einen Schmidfaktor von 0,367. Damit verringert sich der Parameter $\tau_{2}$ erheblich. Dies hat zur Folge, dass durch die hohe aufgelöste Schubspannung auf die trailing Partialversetzung volle Versetzungen im Zwilling erwartet werden können. 


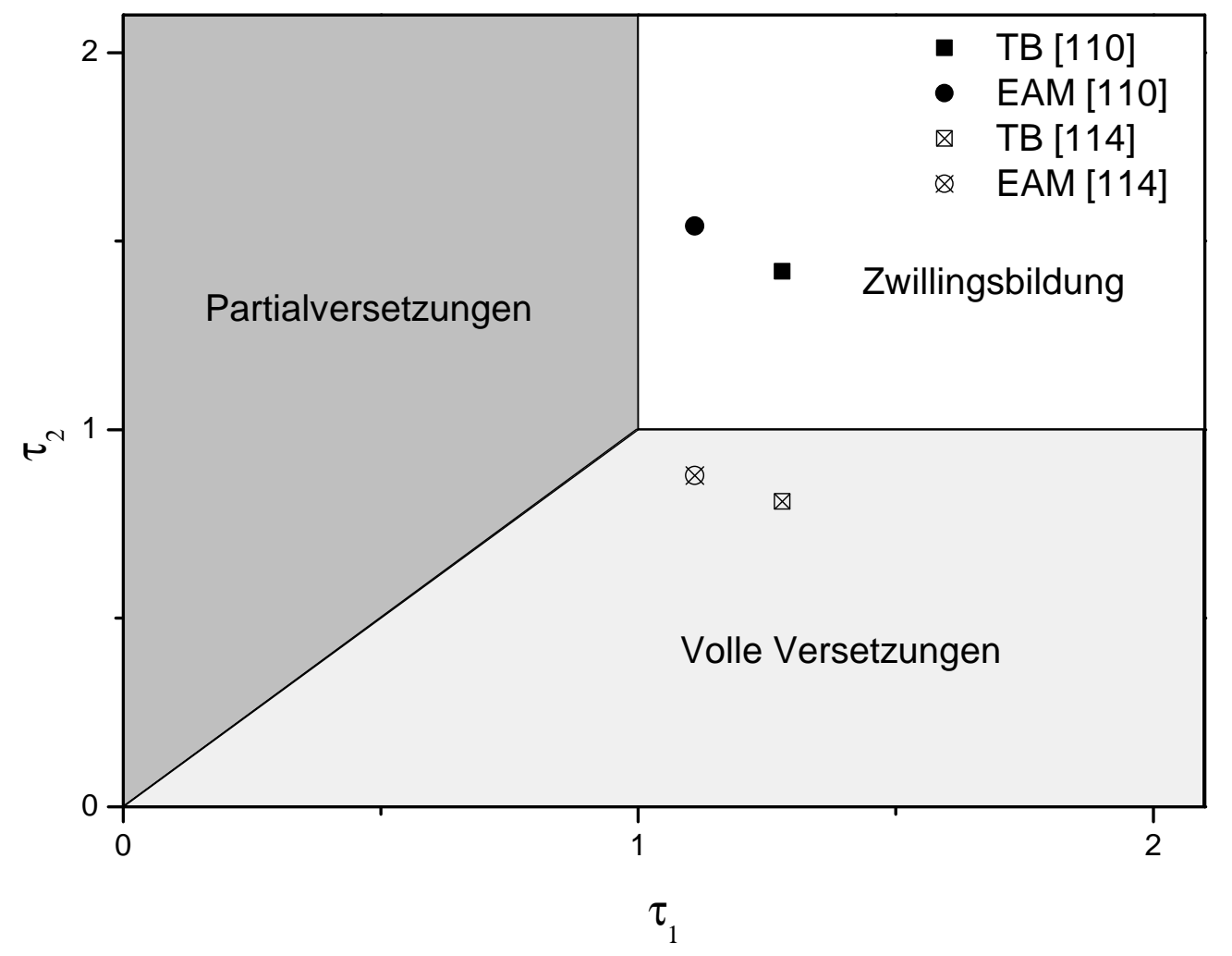

Abbildung 6.7: GSFE Vorhersage der Verformung - Zwei-Parameter-Darstellung für die Verformung nach [61]. Zugversuche an Gold in [110]-Richtung werden in dieser Betrachtung in den Zwillingsbereich eingeordnet. Für einen Zugversuch in [114]-Richtung erwartet man die Verformung über volle Versetzungen. Die Werte für die Stapelfehlerenergie wurden [61] entnommen. 


\section{DISKUSSION}

Obwohl diese experimentell nicht beobachtet werden konnten, gibt das Bruchverhalten Aufschluss über die aufgetretenen Defekttypen. Es konnten zum Beispiel keinerlei Zwillinge in einem bereits umorientierten Bereich beobachtet werden. Dies führt zu dem Schluss, dass der beobachtete Scherbruch durch die Bewegung von vollen Versetzungen verursacht werden musste. Die Einordnung nach dem oben präsentierten Modell stimmt folglich mit der Beobachtung überein.

Für einfache Orientierungen ist die Fähigkeit der Vorhersage durch das Modell an einer Vielzahl von Simulationen bestätigt worden. Auch die in dieser Arbeit beschrieben Ergebnisse lassen sich einfach in das Bild einfügen. Dennoch gibt es in der Betrachtungsweise der GSFE einige Limitierungen. Eine sehr verbreitete experimentelle Beobachtung vom vermehrten Auftreten von Stapelfehlern und Zwillingen in nanokristallinem Aluminium lässt sich nicht einfach mit dem Modell in Einklang bringen [63]. In diesen Proben liegt aufgrund der Mikrostruktur ein komplexer Spannungszustand vor. Das vorliegende Modell lässt für einen solchen Fall aber keine Einordnung zu, da ein einfaches geometrisches Argument dort nicht mehr ausreichend ist. Zudem ist Aluminium ein Material mit einer sehr hohen Stapelfehlerenergie, was im vorliegenden Modell einen Wert für $\tau_{2}$ von unter $0,6 \cdot S_{L} P / S_{T} W$ vorhersagt. Damit wird für eine Vielzahl von Kombinationen von Schmidfaktoren volle Versetzungen erwartet. 


\subsubsection{Klassische Keimbildungstheorie}

Die Beschreibung des Verformungsverhaltens über das Konzept der GSFE stellt eine Möglichkeit dar, die in der Lage ist zumindest qualitativ die Versetzungstypen zu bestimmen. Eine vollständige Beschreibung der Verformung berücksichtigt aber auch die Dynamik der beteiligten Prozesse und deren genaues atomistisches Bild. In diesem Abschnitt soll das Modell der Keimbildung von Versetzungen um diesen Aspekt erweitert und auf die hier vorliegende Geometrie angewendet werden.

Versetzungskeimbildung als thermisch aktivierter Prozess wird schon lange in der Literatur diskutiert. Die Annahme geht davon aus, dass zu jeder Zeit eine Vielzahl von unterkritischen Versetzungskeimen im Volumen enthalten sind, die in ihrer Größe durch die thermische Bewegung fluktuieren. Durch weitere Fluktuationen kann ein solcher unterkritischer Keim eine Größe erreichen, ab der jedes weitere Wachstum mit einer Verringerung der Energie verknüpft ist, den kritischen Radius $\mathrm{R}_{c}$. Dieser Prozess wird, wie in Abbildung 6.8 dargestellt, nicht nur durch materialspezifische Parameter bestimmt, sondern auch durch eine äußere Spannung getrieben. Die Energiebarriere ist für kleine Spannungen am höchsten und verringert sich auf Null für eine bestimmte Spannung. Diese wird athermische Spannung genannt und bezeichnet den Zeitpunkt, ab dem Keime spontan wachsen können. In [71] ist eine explizite Verfahrensweise gegeben, wie man in diesem Modell über die verschiedenen Energiebeiträge eines Keims zu der Keimbildungsrate und damit verbunden zu einer kritischen theoretischen Nukleationsspannung für Versetzungen gelangt. Es ist lediglich nötig, die für die Keimbildung relevanten Energiebeiträge zu identifizieren und eine adäquate Formulierung für diese zu finden. Nach gegenwärtigem Verständnis der heterogenen Versetzungskeimbildung beinhaltet eine vollständige Beschreibung der beteiligten Prozesse auch den Effekt von Oberflächenstufen [73]. In dem von Hirel et. al. [72] präsentierten elastischen Modell werden für die Energiebeiträge die folgenden Formulierungen verwendet:

- die Eigenenergie $\mathrm{E}_{d}$ einer Versetzungslinie für einen halbkreisförmigen Keim an einer Oberfläche

$$
E_{d}=\frac{G b^{2}(2-\nu)}{8(1-\nu)} R\left[\ln \left(\frac{8 m \alpha R}{b}\right)-2\right]
$$

- die Dehnungsenergie $\mathrm{E}_{\epsilon}$ die durch eine Versetzung relaxiert werden kann

$$
E_{\epsilon}=-G b(1+\nu) e \pi R^{2} \cos \theta_{1} \cos \theta_{2} \epsilon
$$




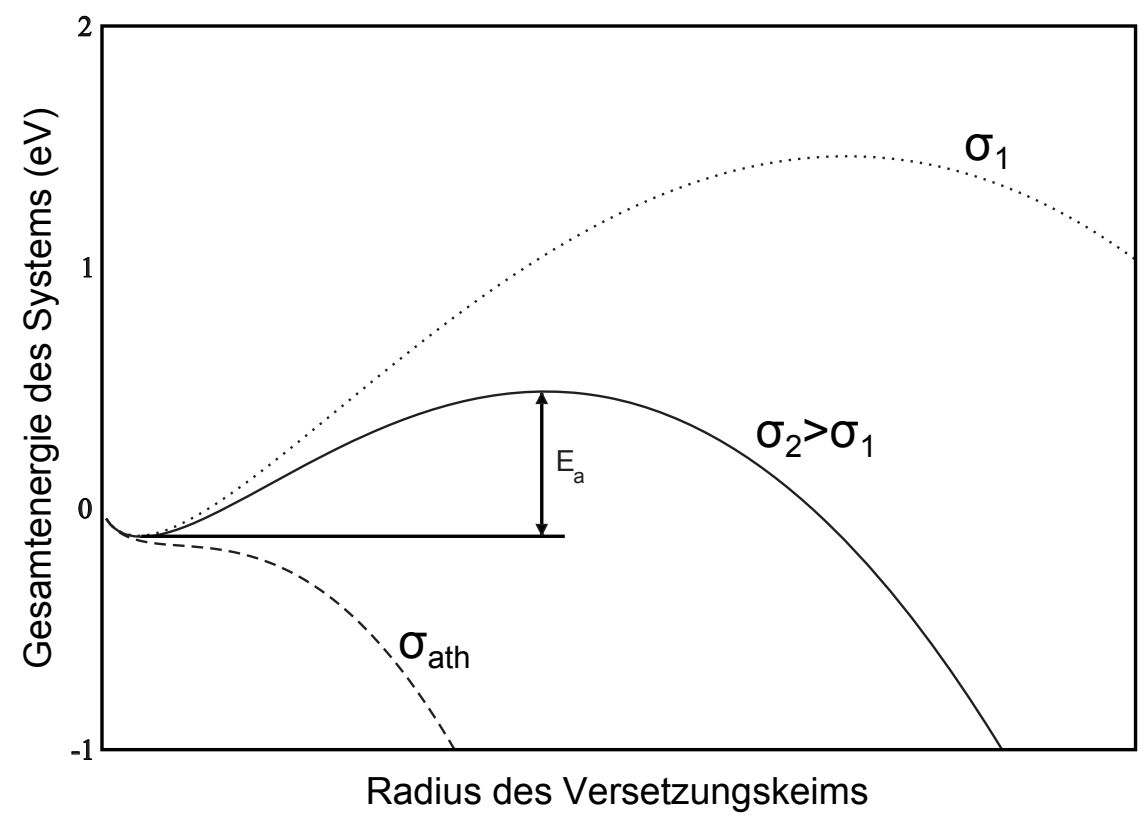

Abbildung 6.8: Kritischer Keimradius im elastischen Modell - Beispielhafter Verlauf der Gesamtenergie in Abhängigkeit von der Versetzungskeimgröße (nach [72]). Für unterschiedliche Spannungen ergeben sich andere Keimgrößen. Ab einer kritischen Spannung erfolg die Keimbildung spontan (gestrichelt gezeichnet).

- der Beitrag $\mathrm{E}_{s L}$ oder $\mathrm{E}_{s T}$ durch den Abbau einer atomaren Oberflächenstufe durch die leading oder trailing Partialversetzung

$$
\begin{aligned}
& E_{s L P}=-(2 / 3) \cdot 2 e R \sigma_{s} \\
& E_{s T P}=-(1 / 3) \cdot 2 e R \sigma_{s}
\end{aligned}
$$

- die Stapelfehlerenergie $\mathrm{E}_{\gamma}$, da beim Durchlaufen einer Partialversetzung ein planarer Defekt erzeugt wird

$$
E_{\gamma}=\gamma \pi e R^{2} / 2
$$

Für die Bildung eines Keims muss folglich die Gesamtenergie

$$
E(R)=E_{d}+E_{\epsilon}+E_{s L T}+E_{\gamma}
$$

aufgebracht werden. Der kritische Radius $\mathrm{R}_{c}$ kann aus so einer Beschreibung heraus berechnet werden, wenn die Stabilitätskriterien erfüllt werden. Diese sind definiert durch 
die Bedingung, dass ein Maximum in der $\mathrm{E}(\mathrm{R})$ Kurve vorliegt. Somit wird sichergestellt, dass bei $\mathrm{R}_{c}$ der Keim stabil ist $\left(\mathrm{dE} /\left.\mathrm{dR}\right|_{R_{c}}=0\right)$ und dass ein Energiegewinn erzielt wird, wenn der Keim in seiner Größe wächst $\left(\mathrm{d}^{2} \mathrm{E} /\left.\mathrm{dR}^{2}\right|_{R_{c}}<0\right)$.

Für die erste durchlaufende Partialversetzung lässt sich der kritische Radius berechnen, indem man die Bestimmungsgleichung für $\mathrm{R}_{c}$ löst. Dazu werden neben den materialspezifischen Größen $\mathrm{G}=27 \mathrm{GPa}, \nu=0,44, \mathrm{~b}=1,66 \cdot 10^{-10} \mathrm{~m}$ und $\gamma=40 \mathrm{~mJ} / \mathrm{m}^{-2}$ auch Parameter verwendet, um die Geometrie möglichst genau widerzugeben. Die Größe $e$ berücksichtigt die Abweichung eines elliptischen Keims vom idealen kreisförmigen Keim und kann aus MD Simulationen bestimmt werden [72]. Da für Gold keine weitere Information über die Keimform vorhanden ist, wird im Weiteren dieser Einfluss vernachlässigt und von einem kreisförmigen Keim ausgegangen. Der Paramter $\alpha$ berücksichtigt die Verzerrungsenergie des Versetzungskerns und beträgt für fcc Metalle circa $\alpha=2$ [71]. Die Winkelabhängigkeit von $\mathrm{E}_{\epsilon}$ berücksichtigt die durch den Schmidfaktor gegebene aufgelöste Schubspannung für die jeweilige Partialversetzung. Der Faktor $m$ wurde in [74] eingeführt, um die Eigenenergie eines nicht kompletten Versetzungsringes beschreiben zu können. Dort wird auch eine Formulierung für $m$ gegeben, die nur von $\nu$ abhängt. Dies führt im Fall von Gold zu $m \approx 0,569$. Experimentell schwierig zugänglich ist zudem der Wert für eine Oberflächenstufe [75]. Hier wird der in [72] gegebene Wert von $\sigma_{s}=5 \cdot 10^{-11} \mathrm{~J} / \mathrm{m}$ verwendet. Rechnungen mit typischen Werten, die sich nach der Vorschrift

$$
\frac{E}{L}=\gamma_{\text {Oberfläche }} \cdot b \approx \frac{\mu b}{10}
$$

ergeben [76], zeigen dabei nur eine sehr kleine absolute Verschiebung der Kurven. Die relativen Beziehungen bleiben davon unberührt [74].

Nimmt man alle Werte zusammen, lässt sich die kritische Keimgröße nach 6.18 für eine Partialversetzung mit dem höchsten Schmidfaktor $\left(\mathrm{m}_{S}=0,471\right)$ berechnen, die eine Oberflächenstufe abbaut und einen Stapelfehler erzeugt. Dafür wird die transzendente Gleichung für $\mathrm{R}_{c}$

$$
R_{c}=\frac{1}{16 \pi(1+\nu) m_{s} \epsilon} \cdot\left\{b(2-\nu)\left[\ln \left(\frac{8 m \alpha R_{c}}{b}\right)-1\right]+\frac{8(1-\nu)}{\mu b}\left(\gamma \pi R_{c}-\frac{4}{3} \sigma_{s}\right)\right\}
$$




\section{DISKUSSION}

für unterschiedliche Dehnungen $\epsilon$ berechnet. Die Dehnung übersetzt sich in der gewählten Geometrie in Spannung mit Hilfe von

$$
\epsilon=\frac{\sigma}{E_{110}}
$$

Für die nachlaufende Partialversetzung muss Gleichung 6.18 umgeschrieben werden. Wurde die Oberflächenstufe noch durch die erste Partialversetzung um zwei Drittel zurückgebildet, wird mit der nachlaufenden Versetzung die Stufe nur um ein Drittel verkleinert. Darüber hinaus liegt die nachlaufende Partialversetzung anders zur Belastungsrichtung, was den anderen Schmidfaktor von $\mathrm{m}_{S}=0,236$ bewirkt. Allerdings wird der planare Defekt abgebaut und die Energie des Gesamtsystems dadurch verringert. Gleichung 6.18 lautet demnach für diese Versetzung

$$
E(R)=E_{d}+E_{\epsilon}+E_{s T P}-E_{\gamma}
$$

Damit ändert sich auch die Gleichung für den kritischen Keimradius zu

$$
R_{c}=\frac{1}{16 \pi(1+\nu) m_{s} \epsilon} \cdot\left\{b(2-\nu)\left[\ln \left(\frac{8 m \alpha R_{c}}{b}\right)-1\right]+\frac{8(1-\nu)}{\mu b}\left(-\gamma \pi R_{c}-\frac{2}{3} \sigma_{s}\right)\right\}
$$

Die dritte Möglichkeit für die Versetzungsbildung ist die Nukleation einer leading Partialversetzung auf einer benachbarten Ebene eines existierenden intrinsischen Stapelfehlers. Für diesen Fall gibt es zwei Möglichkeiten der Oberflächenstruktur und damit verbundene Änderungen für den kritischen Nukleationsradius. Liegt auch an diesem Nukleationspunkt eine Oberflächenstufe vor, gleicht die Bestimmungsgleichung für den kritischen Keimradius der der leading Partialversetzung. Es gibt jedoch einen Unterschied in dem erzeugten Stapelfehler. Für den Fall der zwillingsbildenden Partialversetzung muss nur die Differenzenergie zwischen dem existierenden Stapelfehler $\gamma_{i S F}$ und der Grenzflächenenergie des Zwillings $2 \gamma_{T}$ betrachtet werden. Diese beträgt etwa $10 \mathrm{~mJ} / \mathrm{m}^{-2}$ [30]. Liegt keine Oberflächenstufe vor wird durch die Aktivierung der Versetzung eine Stufe erzeugt. Das bedeutet, dass die Energie für die Zwillingsgrenzfläche und die gebildete Stufe aufgebracht werden muss.

Fasst man die Keimradien in Abhängigkeit von der angelegten Spannung zusammen, gelangt man zu den Verläufen in Abbildung 6.9. In dieser Abbildung sind die Werte für die Nukleation an einer Oberflächenstufe gestrichelt eingezeichnet. Zusätzlich zu den oben dargestellten Möglichkeiten ist es aber auch möglich, dass die Nukleation von 


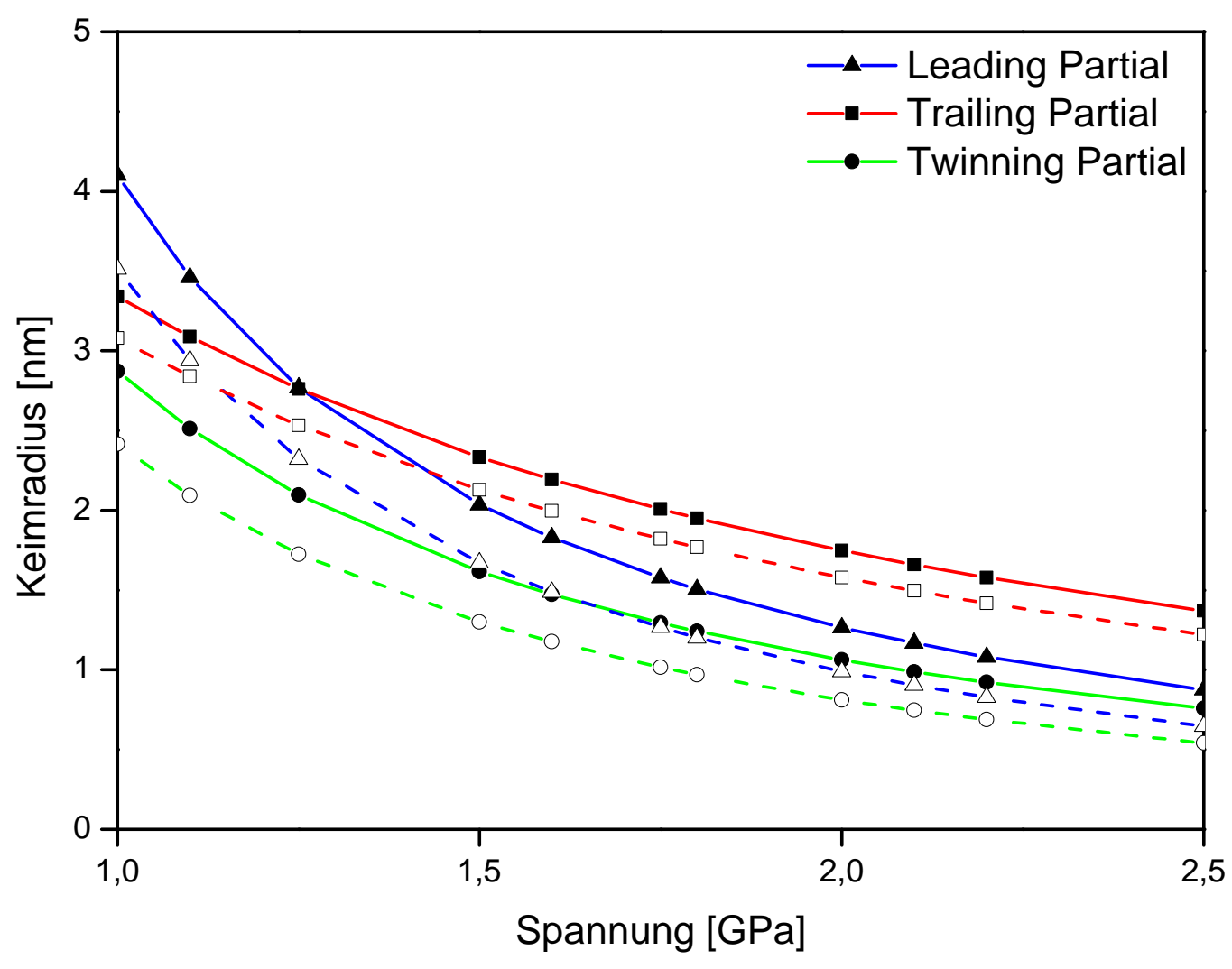

Abbildung 6.9: Kritische Keimradien der Versetzungsnukleation - Kritische Keimradien für die Versetzungsbildung für jeweils die leading, trailing und twinning Partialversetzung in Abhängigkeit von der angelegten Spannung. Durchgezogen mit vollen Symbolen sind die Verläufe für Nukleation jeweils ohne Oberflächenstufen gezeichnet. Gestrichelte Kurven mit offenen Symbolen zeigen der Verlauf für die Nukleation an Oberflächenstufen. 


\section{DISKUSSION}

einer ungestörten Oberfläche ausgeht. In diesem Fall wird durch jede Partialversetzung eine Oberflächenstufe gebildet. Dies bedeutet, dass in die Bestimmungsgleichungen für den kritischen Radius diese Energiebeiträge mit umgekehrtem Vorzeichen eingehen. Die sich daraus ergebenen Keimradien sind mit durchgängiger Linie und geschlossenen Symbolen in Abbildung 6.9 eingezeichnet.

Aus der Auftragung in Abbildung 6.9 wird deutlich, dass bei Erhöhung der Spannung der kritische Keimradius für alle Szenarien und Partialversetzungen kleiner wird. Erkennbar ist weiterhin, dass das Abbauen einer Oberflächenstufe stets dazu führt, dass bei gleicher Spannung ein kleinerer Keim stabil wird. Bei Spannungen von 1 GPa weist die erste Partialversetzung noch einen größeren Keimradius auf als die nachlaufende Partialversetzung. Erst bei Spannungen von 1,25 GPa und mehr dreht sich dieses Verhältnis für die ungestörte Oberfläche um. An Stufen geschieht dies schon bei kleineren Spannungswerten. Das geschilderte Verhalten ist ein Indiz dafür, dass die energetische Bedeutung der Auflösung des Stapelfehlers bei hohen Spannung durch die höhere aufgelöste Schubspannung für die leading Partialversetzung überkompensiert wird. Dies zeigt sich zusätzlich dadurch, dass der Verlauf für die trailing Partialversetzung flacher ist als für die leading. Die Tatsache, dass die twinning Partialversetzung kleinere Keimradien aufweist als die leading Partialversetzung, liegt darin begründet, dass die aufzubringende Energie für die Zwillingsgrenzfläche sehr viel kleiner ist als die eines neuen intrinischen Stapelfehlers. Die Spannungsabhängigkeit des Radius ist aufgrund der gleichen Schmidfaktoren für beide aber gleich und es kommt nicht zum Überschneiden der Kurven.

Mit der Kenntnis über die Keimradien lässt sich nun im Bild von 6.8 die zugehörige Aktivierungsenergie ausrechnen. Dazu wird der kritische Keimradius wieder in die Gleichung 6.18 oder 6.22 eingesetzt. Man erhält damit für die oben skizzierten Ausgangssituationen mit und ohne Oberflächenstufe den spannungsabhängigen Verlauf der Aktivierungsenergie aus Abbildung 6.10. In dieser Grafik wird der Trend aus den kritischen Radien noch einmal deutlich. Die Aktivierungsenergie ist bei kleinen Spannung für die erste Partialversetzung am höchsten. Die nachlaufende Versetzung ist aufgrund der oben beschriebenen Situation durch das Aufheben des Stapelfehlers in ihrer Aktivierung begünstigt. Die kleinste Energiebarriere weist in diesem Modell die zwillingsbildende Partialversetzung auf. Alle Energiebarrieren können herabgesetzt werden, indem 


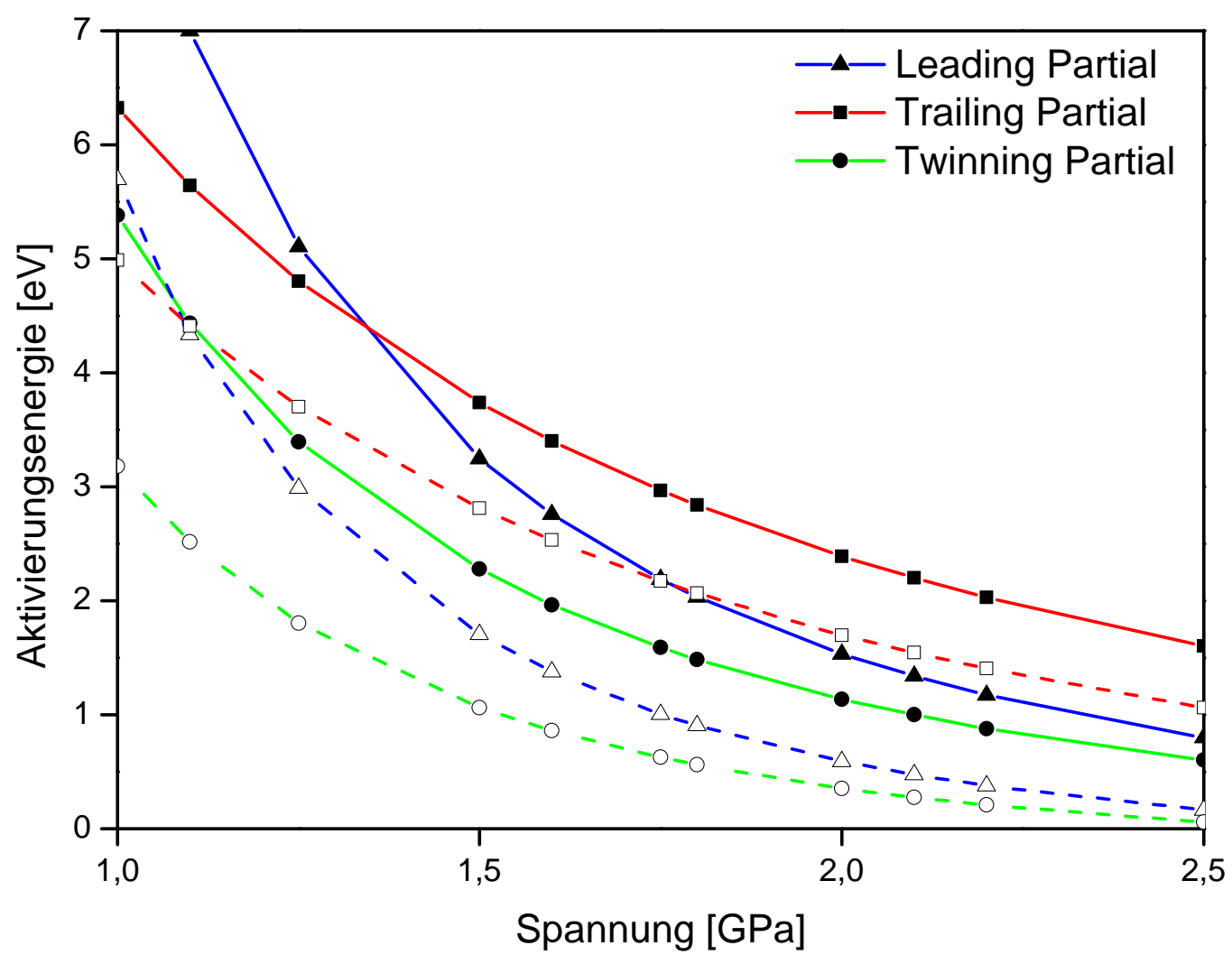

Abbildung 6.10: Energie der kritischen Versetzungskeime - Energien der kritischen Keime in Abhängigkeit der angelegten Spannung. Die durchgezogenen Linien geben den Energieverlauf für die Keime wieder, die sich an der ungestörten Oberfläche bilden. Gestrichelt sind die Energien dargestellt, die zu einem Versetzungskeim an einer Oberflächenstufe gehören. 


\section{DISKUSSION}

man die Nukleation an Oberflächenstufen zulässt. Dies ist durch die gestrichelten Linien in Abbildung 6.10 dargestellt.

\section{Beschreibung der Verformung über Nukleationsraten}

Die Argumentation für die Keimbildung in nanoskaligen Materialien endet in der Literatur mit der Betrachtung der Aktivierungsenergie oder der typischen kritischen Keimradien [77, 78]. Die Beschreibung der Prozesse allein über die Aktivierungsenergie ist allerdings nicht ausreichend, um auf das reale Auftreten im Experiment schließen zu können. Vielmehr muss betrachtet werden, dass die Nukleation von trailing und twinning Partialversetzung nur dann stattfinden kann, wenn vorher eine leading Partialversetzung gebildet wurde, die Prozesse sind insoweit nicht voneinander unabhängig. Man kann allerdings die Kenntnis der zuvor gewonnenen Energiebarrieren dafür verwenden eine Nukleationsrate zu berechnen [71]. Für einen thermisch aktivierten Prozess gilt dabei für die Nukleationsraten

$$
J=\omega n_{c}
$$

Hier beschreibt $\omega$ die Frequenz mit der dem Keim benachbarte Atome beitreten können und ist gegeben durch [71]

$$
\omega=\frac{8 \pi R_{c} \nu_{D}}{b},
$$

wo $\nu_{D}$ die Debye-Frequenz ist. Die Gleichgewichtskonzentration von Keimen bei gegebener Aktivierungsenergie beträgt

$$
n_{c}\left(E_{a}, T\right)=n_{0} \exp \left(-\frac{E_{a}}{k_{B} T}\right)
$$

Entscheidend in der Formel ist der Vorfaktor $\mathrm{n}_{0}$, der angibt, an wie vielen äquivalenten Plätzen sich ein Keim bilden kann. Für die in dieser Arbeit getesteten Drähte kann man den Grenzfall für die leading Partialversetzung bestimmen. Dieser liegt dann vor, wenn sich Oberflächenstufen auf jeder der zwei aktiven $\{111\}$-Ebenen über die gesamte Drahtlänge von $\mathrm{L}=5 \mu \mathrm{m}$ befinden. Für diesen Fall ist $\mathrm{n}_{0}$ gegeben durch

$$
n_{0}=2 \cdot \frac{L}{d_{\{111\}}}=4,26 \cdot 10^{4}
$$

Diese einfache Annahme trifft aller Wahrscheinlichkeit nach nicht ganz den Oberflächenzustand der verwendeten Drähte, von denen die genau Stufenanzahl nicht bekannt ist. 
Allerdings kann man aus der statistischen Abschätzung der Nächste-Nachbar-Abstände aus Abschnitt 5.1 ersehen, dass Stapelfehler mit einem mittleren Abstand von etwa 8 $\mathrm{nm}$ unabhängig voneinander gebildet werden können. Geht man davon aus, dass dies Plätze waren, an denen Oberflächenstufen vorhanden sind, erhält man für einen Draht mit $5 \mu \mathrm{m}$ Länge einen Wert von $\mathrm{n}_{0} \approx 600$. Dieser kann als untere Nachweisgrenze aufgefasst werden und verdeutlicht, dass jederzeit genug Stufen an der Oberfläche vorhanden sind, um als Nukleationspunkte für die leading Partialversetzung zu fungieren. Die generellen Trends der Nukleationsraten werden darüber hinaus nicht wesentlich durch die exakte Wahl des Wertes innerhalb der Grenzen verändert.

Für die trailing und twinning Partialversetzung ist die Anzahl der äquivalenten Plätze nicht einfach auszurechnen. Vielmehr ist für diese Fälle das Vorhandensein eines intrinsischen Stapelfehlers vonnöten. Dieser wird aber erst durch die leading Partialversetzung gebildet. Damit wird der Vorfaktor zeitabhängig und steigt mit zunehmender Zahl von intrinsischen Stapelfehlern an. Der Einfachheit halber wird hier zum Vergleich der Nukleationsraten ein Stadium der Verformung angenommen, in dem schon 100 Stapelfehler vorhanden sind. Demnach ist $\mathrm{n}_{0}$ für diese Fälle genau 100. Der Einfluss der genauen Größe dieses Vorfaktors kann gut abgeschätzt werden, da er nur linear in die Nukleationsraten eingeht. Zu diesem Zweck wird für die beiden Fälle der trailing und der twinning Partialversetzung eine Einhüllende gegeben, die den Bereich von 1 bis 1000 vorhandenen Stapelfehler abdeckt. Diese Werte sind als absolut unterste bzw. oberste Grenze in den Experimenten zu verstehen. Der spannungsabhängige Verlauf der Nukleationsraten nach diesen Vorgaben ist in Abbildung 6.11 gezeigt.

Erkennbar ist aus dieser Darstellung heraus, dass in allen Fällen die Nukleationsrate über mehrere Größenordnung mit der Spannung ansteigt. Ab einer gewissen Spannung kommt man dann in Bereiche, in denen die Nukleationsraten messbar sind. Dieser Bereich ist in Abbildung 6.11 grau hinterlegt und als "experimentell zugänglich"markiert. Mit Hilfe dieser Auftragung ist man nun in der Lage, experimentell beobachtetes Auftreten von Defekten eine Wahrscheinlichkeit nach dem oben präsentierten Modell zuzuordnen.

Dabei wird im Experiment die Spannung schrittweise erhöht, sodass man sich auf der Spannungsachse nach rechts bewegt. Der erste mögliche Prozess, der mit einer experimentell zugänglichen Häufigkeit auftritt, ist die Nukleation einer leading Partialversetzung an einer Stufe bei Punkt P1 und einer Spannung von etwa 1,7 GPa. Dieser Wert 


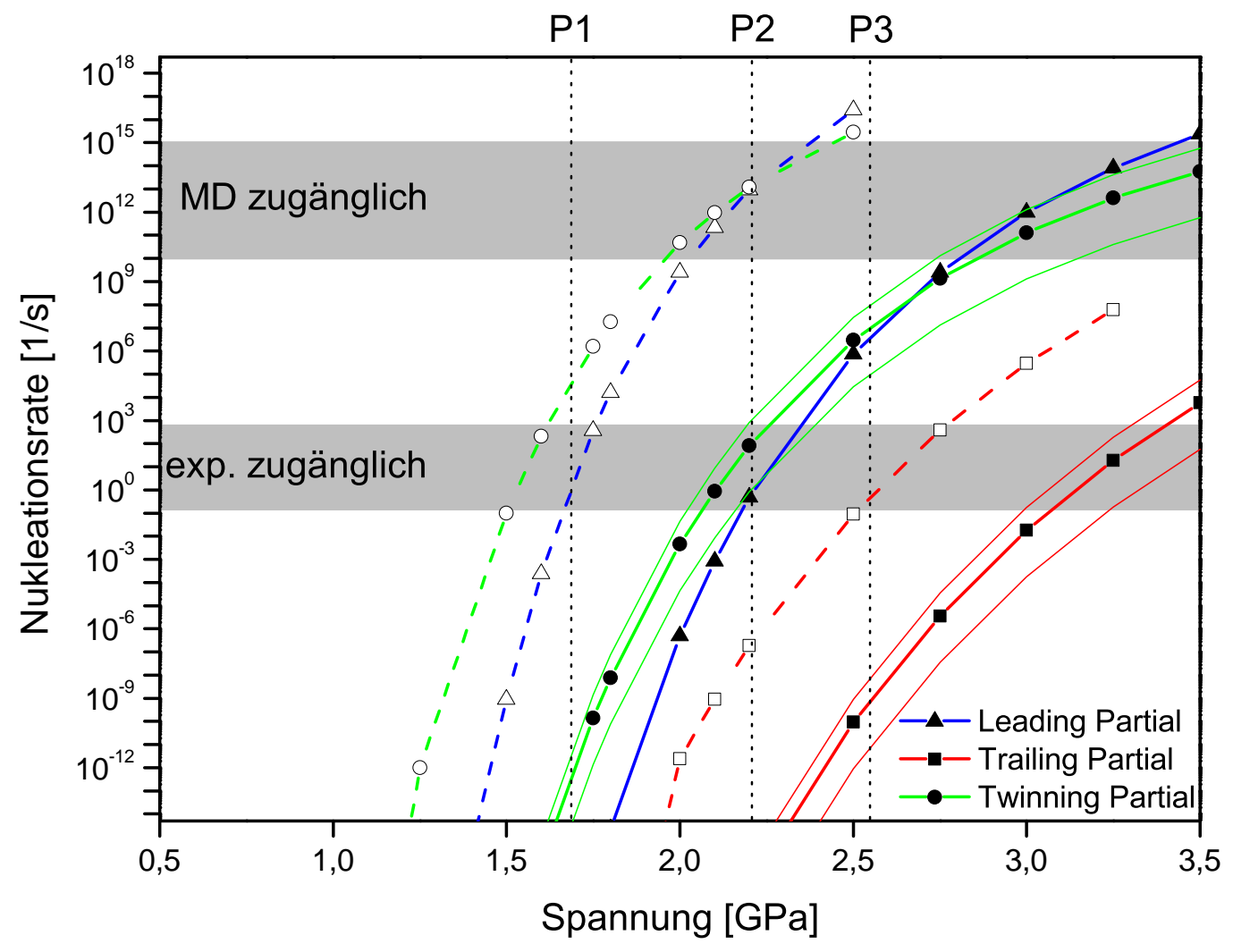

Abbildung 6.11: Nukleationsraten für Partialversetzungen - Nukleationsraten für verschieden Partialversetzungen in Abhängigkeit von der angelegten Spannung. Die durchgezogenen Linien beschreiben den Verlauf für die Nukleation an einer ungestörten Oberfläche. Für die twinning und trailing Partialversetzungen ist der Einfluss der Anzahl möglicher Nukleationsplätze eingezeichnet (vgl. dazu den Text). Die gestrichelten Linien geben den Verlauf für Nukleation an Oberflächenstufen wieder. Grau unterlegt sind Nukleationsraten, die im Experiment bzw. typischer Weise bei MD Simulationen erreicht werden. 
liegt etwas über der im Experiment an Nanodrähten bestimmten Streckgrenze bei 1,1 bis 1,2 GPa [24]. Die Spannung, die lokal an einer Oberflächenstufe auftritt, kann mitunter aber höher sein als die außen angelegte Spannung [79]. Dies bedeutet, dass die Nukleationsspannung zwar global noch nicht erreicht ist, aber an Oberflächenstufen die Spannungskonzentration ausreicht, um eine leading Partialversetzung zu aktivieren. Die gegebenen Spannungswerte sind demnach nicht direkt mit gemessenen Werten zu vergleichen.

Bei der Spannung, bei der die erste Nukleation einer Partialversetzung zu erwarten ist, ist ein weiterer Prozess mit höherer Nukleationsrate zu erwarten, die Aktivierung einer zwillingsbildenden Partialversetzung auf der benachbarten Ebene. Allerdings muss dafür auch dort eine Oberflächenstufe vorhanden sein. Diese Tatsache bedingt, dass spontan ein Zwilling gebildet wird, bis keine benachbarten Oberflächenstufen mehr vorhanden sind. Die Breite der Verformungszwillinge ist somit nach diesem Modell festgelegt durch den Bereich, in dem benachbarte Oberflächenstufen vorliegen. Da dies eine Eigenschaft des Drahtes ist, ist auch die Zwillingsbreite vordefiniert.

Das beschriebene Verformungsverhalten dauert solange an, bis alle existierenden Oberflächenstufen abgebaut wurden. Der dann als nächstes begünstigte Prozess erfordert eine höhere Spannung und tritt bei Punkt P2 auf. Ab 2,2 GPa können auch an ungestörten Oberflächen Partialversetzungen nukleiert werden. Ist dieser Zustand erreicht, kann auf einer benachbarten Ebene eine zwillingsbildende Partialversetzung erzeugt werden. Dieser Prozess hat eine sehr hohe Nukleationsrate und würde bei gleich bleibendem Spannungszustand zu einem steten Wachstums des Zwillings führen.

Die Aktivierung der nachlaufenden Partialversetzungen setzt zwar ebenso die Existenz eines Stapelfehlers voraus, wird aber erst bei sehr viel höheren Spannungen erwartet. In Abbildung 6.11 ist dies erst an Punkt P3 der Fall. Dort liegt eine Spannung von etwas über 2,5 GPa vor und benötigt dabei die weitere Reduktion einer Oberflächenstufe. Die Nukleation an einer ungestörten Oberfläche ist erst bei sehr viel höheren Spannungen messbar und folglich im Experiment noch weniger wahrscheinlich.

\section{Einordnung der experimentellen Ergebnisse und der Literatur}

Zusammenfassend lassen sich die experimentell gefundenen Ergebnisse sehr gut in das vorher beschriebene Modell integrieren. Es konnte in Abschnitt 5.1 gezeigt werden, dass 


\section{DISKUSSION}

die Verformung der Nanodrähte mit der Nukleation von Stapelfehlern auf verschiedenen $\{111\}$-Ebenen beginnt. Es ist anzunehmen, dass die Partialversetzungen gebildet werden, die den höchsten Schmidfaktor aufweisen. Dies deckt sich mit den Berechnungen anhand des Modells. Die Nukleation geschieht dabei an unterschiedlichen Positionen im Draht und ist über die gesamte Länge des verformbaren Bereiches verteilt. Die Punkte sind dann dadurch ausgezeichnet, dass Oberflächenstufen vorhanden waren. Dies ist in TEM Untersuchungen an Gold-Nanodrähten auch experimentell bestätigt worden $[80,81]$.

Das verteilte Auftreten von Zwillingen an verschiedenen Stellen im Draht ist in diesem Zusammenhang dadurch erklärbar, dass dort benachbarte Oberflächsenstufen vorlagen. Die Beobachtungen decken sich insofern mit dem Modell, als dass in diesen Bereichen kein ausgeprägtes Zwillingswachstum beobachtet wurde. Vielmehr bleiben existierende Zwillinge in ihrem Volumen relativ konstant. Bei weitergehender Verformung tritt dann meist zentral in der Mitte des Drahtes ein wachsender Zwilling auf. Dort wird auch ohne existierende Oberflächenstufen die kritischen Spannungen erreicht, um das Wachstum voranzutreiben. An den Übergangspunkten vom Zwilling zur Matrix entsteht aufgrund der Formänderung eine Spannungskonzentration [82]. Diese führt dazu, dass dort bevorzugt weitere zwillingsbildende Partialversetzungen nukleiert werden. Mit der damit verbundenen Formänderung wird dann der Spannungszustand komplexer, sodass das Modell über das Bruchverhalten innerhalb des Zwillings keine verlässliche Auskunft mehr liefert.

Das vorhergesagte seltene Auftreten der trailing Partialversetzung kann, wie in Abschnitt 5.1 beschrieben, durch das Experiment bestätigt werden. Da es dennoch vereinzelt beobachtet wurde, ist denkbar, dass eine Stufe existierte, die die Nukleation überhaupt ermöglichte und darüber hinaus die lokalen Spannungen hoch genug waren, um eine messbare Nukleationsrate zu erhalten.

In der Einleitung zu dieser Arbeit wurden zwei Gruppen erwähnt [26, 25], die zum Teil ein abweichendes Verhalten im beobachteten Mechanismus der Verformung gesehen haben. Dort trat während der Verformung eine geschlossene Zwillingsbildung über einen langen Bereich im Draht auf, die zu einer fast kompletten Reorientierung der Probe führte. Dieses Verformungsverhalten konnte in dieser Arbeit nicht in einer derart ausgeprägten Form beobachtet werden. Allerdings stammten die verwendeten Proben 
aus unterschiedlichen Herstellunsgprozessen und Anlagen. Diese Tatsache könnte eine unterschiedliche Oberflächenstruktur der Probe zur Folge haben. Diese ist in dem obigen Modell jedoch maßgeblich für die Nukleation der ersten Partialversetzungen. Neben diesem Unterschied in den Proben ist auch die Steifigkeit der verwendeten Verformungsapparaturen nicht identisch. Eine starke Nachgiebigkeit im Versuchsaufbau kann dazu führen, dass Formänderungen einfacher in einem veränderten Spannungszustand in der Probe resultieren. Dies kann unter Umständen sonst gleichberechtigte Prozesse unterschiedlich stark begünstigen oder unterbinden [27].

Eine weitere Tatsache lässt sich direkt aus der Grafik in Abbildung 6.11 ablesen. In MD Simulationen ist die Dauer des Experiments meist auf den Bereich von Nanosekunden beschränkt. Dies liegt daran, dass die Bewegung der Atome auf einer Zeitskala stattfindet, die im Pikosekundenbereich liegt. Möchte man die Wechselwirkung der Atome vollständig beschreiben, sind damit selbst bei vielen Simulationsschritten nur kurze Zeitlängen möglich. Für die Simulation von plastischer Verformung ist es dagegen notwendig, innerhalb dieser kurzen Zeit alle Versetzungen zu nukleieren. In einer einfachen Abschätzung mit einer Simulationsdauer von einer Nanosekunde, in der 10 Versetzungen gebildet werden sollen, braucht man eine Nukleationsrate von $1 \cdot 10^{10}$ Versetzungen pro Sekunde. Dies ist nach Abbildung 6.11 nur dann möglich, wenn man sehr hohe Spannungen einsetzt. Im vorliegenden Beispiel sind diese Nukleationsraten bei Spannungen von etwa 2,8 GPa für den perfekten Kristall ohne Stufen erreicht. Dies deckt sich mit tatsächlich ermittelten Werten für die Verformung von Nanodrähten in MD Simulationen [83, 84]. Dabei wird aus dem Verlauf der Kurven für die zwillingsbildende und leading Partialversetzung deutlich, dass es durchaus bei höheren Spannungen zu einem Mechanismuswechsel kommen kann. Daher sind die beobachteten Ergebnisse und Statistiken aus MD Simulationen nicht unmittelbar auf das Experiment übertragbar.

Physikalische Limitierungen sind dem Modell dadurch gegeben, dass in das elastische Bild immer makroskopische Größen einfließen müssen [85]. Insbesondere wird dies am Beispiel der Linienenergie einer Versetzung deutlich. Diese Energie ist nur dann sinnvoll definiert, wenn die Atome sich in einer linienhaften Konfiguration befinden, die der Annahme ähnelt. Die Beschreibung des Randbereichs eines thermischen fluktuierenden Versetzungskeims ist daher unter Umständen nicht hinreichend genau genug. Es ist sicherlich schwierig die Stapelfehlerenergie auf einer Fläche von einigen $b^{2}$ mit 


\section{DISKUSSION}

Sicherheit zu bestimmen. Allerdings wird die verwendete Energie aus Experimenten gewonnen, die ähnliche Größenordnungen verwenden. Eine typische Verfahrensweise ist, die Aufspaltungsweite von Partialversetzung mit dem Elektronenmikroskop zu erfassen [86].

\section{Statistik der Stapelfehlerverteilung}

An dieser Stelle soll die statistische Natur der Stapelfehlerverteilung, wie sie in Kapitel 5.1 ermittelt wurde, diskutiert werden. Sollten die Defekte alle unabhängig von einander mit gleicher Wahrscheinlichkeit an jedem Ort entstanden sein, dann gehorcht die Zahl der beobachteten Defekte einer Poissonverteilung. Die Verteilung der Abstände zwischen den Defekten wird dann nur über einen Parameter in der Exponentialverteilung bestimmt. Dies gilt auch dann, wenn Oberflächenstufen von vornherein auf der Oberfläche vorhanden sind. In diesem Fall wären diese ebenfalls poissonverteilt. Die Verteilungsfunktion ist gegeben durch

$$
f(x)=N \cdot \lambda_{f i t} e^{-\lambda_{f i t} x}
$$

Erlaubt wird zusätzlich ein Normierungsfaktor N, der dadurch gerechtfertigt ist, dass nicht die relativen Häufigkeitender jeweiligen Abstände genommen werden, sondern vielmehr die Häufigkeit eines Auftretens innerhalb einer Abstandsklasse. Dadurch wird der einzelne Abstandswert in seiner Häufigkeit übermäßig stark gewichtet.

Die Daten aus 5.6 wurden mit der oben aufgeführten Verteilungsfunktion angepasst. Das wesentliche Ergebnis dieser Berechnung ist der Parameter $\lambda_{f i t}$, der Auskunft über die Rate gibt, mit der ein Defekt entstehen kann. Zudem erhält man den Normierungsfaktor N; dieser sollte die Größe der Abstandsklasse widerspiegeln. Für den Parameter $\lambda_{\text {fit }}$ wurde durch die vorgenommene Anpassung der Wert 0,04755 ermittelt. In der Exponentialverteilung kann man den Kehrwert des Parameters $\lambda_{f i t}$ allerdings auch als mittleren Abstand zwischen zwei Ereignissen auffassen. Aus der ermittelten Gesamtzahl der Defekte lässt sich ein $\lambda$ ermitteln, dass diesen experimentell gewonnenen mittleren Abstand beschreibt. Er beträgt bei dem ausgewerteten Drahtstück der Länge $888 \mathrm{~nm}$ :

$$
\begin{array}{r}
\lambda=\frac{64}{888 \mathrm{~nm}}=0,0721 / \mathrm{nm} \\
1 / \lambda=\bar{d}=13,9 \mathrm{~nm}
\end{array}
$$




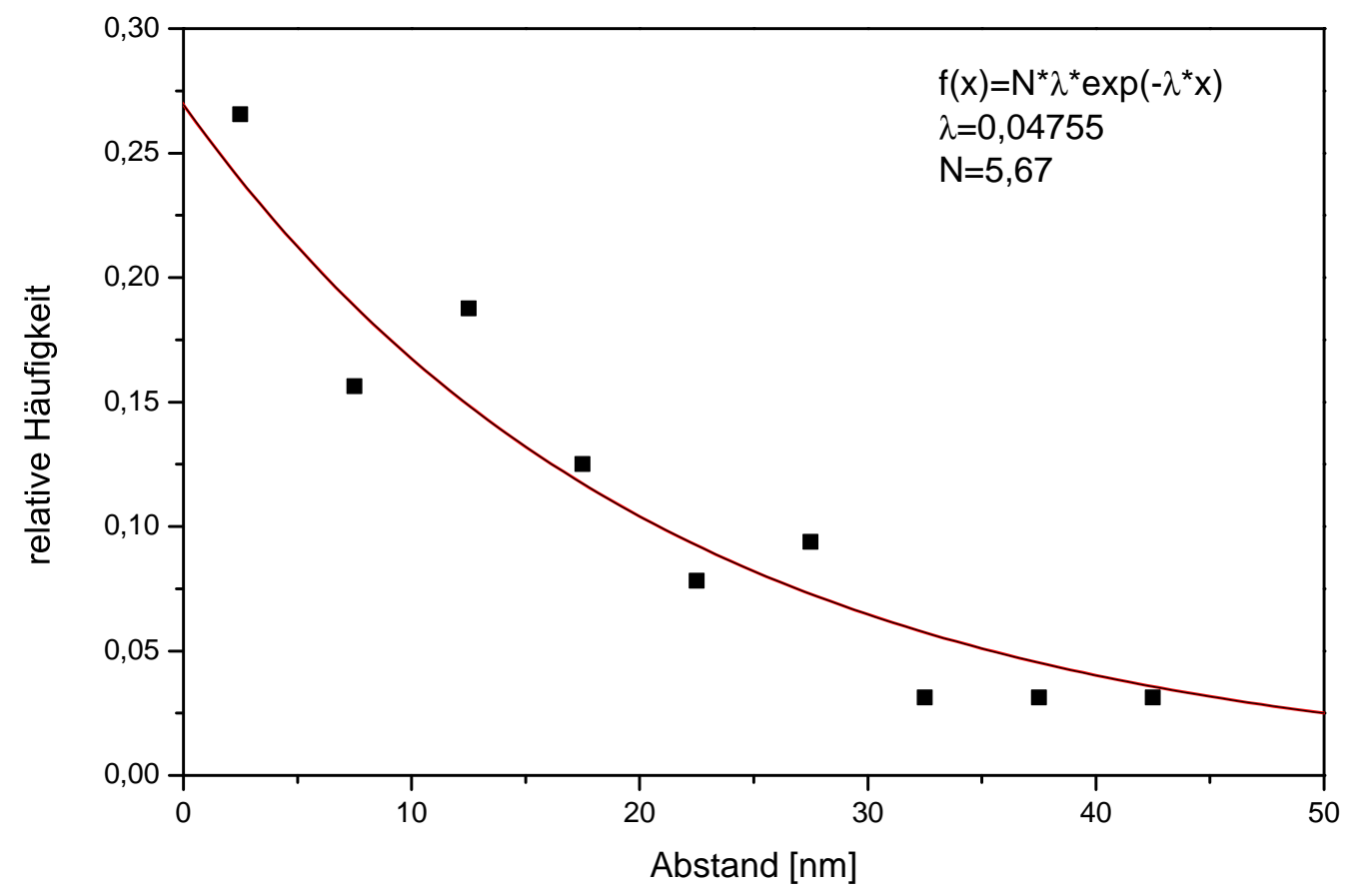

Abbildung 6.12: Untersuchung der Defekte auf statistische Verteilung - Anpassung der Abstandsverteilung an eine Exponentialverteilung 


\section{DISKUSSION}

Die Anpassung an die Messwerte liefert einen mittleren Abstand von

$$
\bar{d}_{f i t}=1 / \lambda_{f i t}=21,05 \mathrm{~nm} .
$$

Dieser Abstand ist um etwa 50\% größer als der durch Auszählen ermittelte Wert. Demnach sind für eine Exponentialverteilung die großen Abstände überrepräsentiert.

Der Normierungsfaktor beträgt nach der Anpassung $\mathrm{N}=5,67$, was gut mit der gewählten Klassenbreite von $5 \mathrm{~nm}$ korrespondiert.

Dass das räumliche Auftreten der Defekte gut durch eine Exponentialverteilung beschrieben werden kann, lässt eine Aussage über mögliche Wechselwirkung zwischen bestehenden Defekten zu. Die wesentliche Annahme hinter der Verteilung ist nämlich, dass ein Poissonprozess vorliegt. Die dafür nötigen Voraussetzungen lassen sich in drei Punkten zusammenfassen:

- Es muss eine gewisse Seltenheit vorliegen, dass heißt, dass Bereiche vorliegen müssen, in denen kein Defektauftreten zu beobachten ist.

- Die Auftrittswahrscheinlichkeit ist konstant.

- Das Auftreten eines Defektes hat keinen Einfluss auf nachfolgende Prozesse.

Der letzte Punkt dieser Aufstellung ist von besonderer Bedeutung für diese Arbeit. Man kann ihn dahingehend verstehen, dass die Nukleationspunkte der ersten Partialversetzungen rein statistisch verteilt sind. Demnach gibt es auch keine Wechselwirkung zwischen den Nukleationspunkten der ersten Partialversetzung. Dies deckt sich mit der Annahme aus den vorherigen Abschnitten, wonach die Nukleation der ersten Partialversetzung an Oberflächstufen stattfindet, die sich zu Beginn der Versuches auf der Drahtoberfläche befinden. Es ist anzunehmen, dass diese Stufen - bedingt durch den Wachstumsprozess - zufällig auf der Oberfläche verteilt sind. Damit spiegelt die Verteilung der Stapelfehler letztendlich die Verteilung der Oberflächenstufen wider.

\subsubsection{Analyse der Zwillingsbildung}

Während des Zugversuches ist es wiederholt zum Auftreten von Zwillingen im Draht gekommen. Dabei werden diese, wie in Abschnitt 5.1.3 gezeigt, durch die Bildung von Stapelfehlern auf benachbarten Ebenen erzeugt. In einem speziellen Fall konnte sogar 
das Wachstum der Zwillingslamelle in ihrer Dicke direkt beobachtet werden. Bemerkenswert ist beim Auftreten der Zwillinge, dass dieses stets nur auf einer \{111\}-Ebene zu beobachten war. Eine Verzwillingung auf beiden Gleitsystemen kam in keinem untersuchten Fall vor. Dieser Befund generiert die Frage, ob es lediglich ein intrinsischer Effekt der Zuggeometrie ist, oder ob äußere Faktoren die Zwillingsbildung beeinflussen. Aus der Theorie ist bekannt, dass die Zwillingsbildung durch das benachbarte Nukleieren von Partialversetzungen auf beiden Gleitsystemen möglich ist. Dieser Fall ist demnach komplett symmetrisch und es ist kein Unterschied zu erwarten. Auch zeigt die Auflistung der aktiven Gleitsysteme in 2.1, dass bei exakter Belastung in [110]-Richtung zwei Gleitsysteme aktiv sein sollten. Ausgehend von dieser Feststellung bleibt nur die Möglichkeit, dass das ungleiche Auftreten von Zwillingen ein Prozess ist, der geometrisch bedingt ist. Vorstellbar wäre zum Beispiel, dass sobald sich der erste Zwilling auf einem der beiden Gleitsysteme gebildet hat, die andere Möglichkeit unterdrückt wird. In Abschnitt 5.1 wurde aufgezeigt, dass ein Stapelfehler für die Bewegung von Partialversetzungen kein effektives Hindernis darstellt. Eine Zwillingslamelle jedoch stellt sehr wohl ein Hindernis dar, wie hinreichend aus der Literatur bekannt ist (vgl. dazu zum Beispiel [87] und [88]). Damit ist eine Unterdrückung der Zwillingsbildung in eine

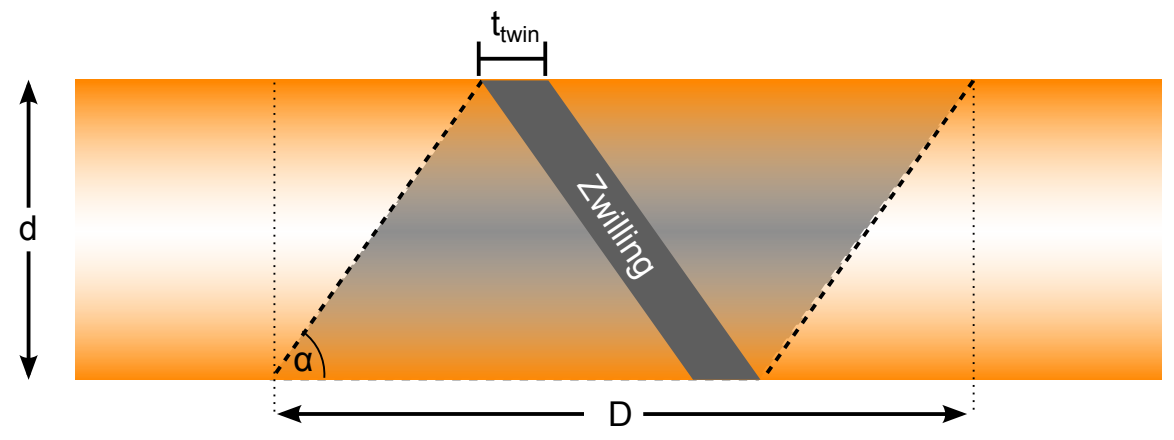

Abbildung 6.13: Schemazeichnung des Einflusses einer Zwillinsglamelle - Durch das Vorhandensein einer Zwillingslamelle wird die Versetzungsbewegung effektiv gehemmt und es entsteht ein verbotener Bereich für die Zwillingsbildung auf einem anderen Gleitsystem.

andere Richtung erklärbar. Allerdings beschränkt sich dieses Unterdrücken auf einen geometrischen Bereich um die vorhandene Zwillingslamelle mit der Breite von

$$
D=3 d \cdot \cot \alpha+t_{t w i n}
$$




\section{DISKUSSION}

Um nach diesem Bild eine Unterdrückung des Zwillingswachstums auf der alternativen $\{111\}$-Ebene über die gesamte Drahtlänge zu erreichen, wäre folglich ein Mindestabstand zwischen zwei Zwillingen nötig, der genau D entspricht. Für einen $100 \mathrm{~nm}$ breiten Draht ist diese Größe bei einer Zwillingslamelle vernachlässigbarer Dicke $\mathrm{D} \approx 210$ nm. Vergleicht man diesen Abstand mit dem mittleren Abstand zwischen den Stapelfehlern, der an einem Beispiel zu etwa $8 \mathrm{~nm}$ bestimmt werden konnt, wird klar, dass dieser Wert sehr groß ist. Es ist somit durchaus vorstellbar, dass aufgrund der Einschränkungen in der Geometrie die Zwillingsbildung eine Unterdrückung erfährt. Dieses Unterdrücken bedeutet dann, dass Versetzungen direkt an der Zwillingsgrenze beobachtet werden müssten sofern deren Gleitebene den existierenden Zwilling schneiden. Ein solches Phänomen wurde jedoch in keinem Draht beobachtet. Die genannten Überlegung lassen darauf schließen, dass der Zugversuch in Fällen, in denen Zwillinge auftraten, nicht perfekt symmetrisch abgelaufen ist. Diese Misorientierung hätte zur Folge, dass ein Gleitsystem eine leicht erhöhte Schubspannung spürt. In Kombination mit einer Spannungskonzentration an dem existierenden Stapelfehler [89] oder Zwilling führt dies zum weiteren Wachstum des Zwillings. Dieser Prozess ist selbstverstärkend, da durch die Formänderung, die mit der Verzwillingung verbunden ist, auch eine erhöhte Spannungskonzentration an den Tripelpunkten Zwilling-Matrix-Oberfläche einhergeht. Diese kann ein weiteres Wachstums des Zwillings begünstigen [90].

\subsubsection{Galliumschädigung}

Die Bestrahlung der Drähte mit Gallium und die damit verbundene Oberflächenschädigung kann mit Hilfe des Nukleationsmodells neu interpretiert werden. Bei der Beobachtung der Verformung ist aufgefallen, dass der Beginn der Verformung sich in dem bestrahlten Gebiet ereignet. Erklärt wurde der Sachverhalt über die kleinere Querschnittsfläche und die dadurch erhöhte Spannung. Neben dieser geometrischen Überlegung ist aber auch ein anderer Grund denkbar. Durch den Erosionsprozess beim Auftreffen der Ionen auf der Oberfläche des Drahtes entstehen dort eine Vielzahl von Punkten, die in ihrer Struktur von der perfekten Oberfläche abweichen [91]. Diese Punkte können als Stufen verstanden werden, an denen eine Spannungskonzentration vorliegt. Darüber hinaus sind dies potentielle Punkte, die durch die Nukleation einer Versetzung in ihrer Energie abgesenkt werden, und damit die Keimbildung dort bevorzugt stattfinden lassen. Die Nukleationsraten in diesem Bild entsprechen damit den gestrichelten 
Verläufen in Abbildung 6.11. Dehnt sich die Oberflächenschädigung sogar über einen größeren Bereich aus, ist vorstellbar, dass auch auf benachbarten Ebenen Oberflächenstufen vorhanden sind. Dies begünstigt die Zwillingsbildung in diesen Bereichen. In der Tat sind innerhalb des bestrahlten Bereiches auch Zwillinge beobachtet worden (vgl. dazu Abbildung 5.34). Somit ist klar, dass kein Wechsel im Verformungsverhalten zu erwarten ist und die Ergebnisse gut den Vorhersagen des Modells folgen.

\subsubsection{Einfluss des Spannungszustandes}

Im Modell der klassischen Keimbildung ist es nun ebenso möglich, einen anderen Spannungszustand zu betrachten. Im Rahmen dieser Arbeit wurde neben der Zuggeometrie auch eine Biegegeometrie zur Verformung verwendet. Diese ist vom Spannungszustand im Draht her sicherlich komplex und daher nicht einfach in das Modell zu integrieren. Allerdings kann man einen Grenzfall der Belastung betrachten und somit ein Gefühl für die Auswirkung eines veränderten Spannungszustandes erlangen. Dieser Grenzfall wäre die Reduktion des komplexen Biegeversuches auf einen Druckversuch. Hier ist der Spannungszustand bei der Verbiegung überall homogen und mögliche Beiträge durch Verbiegung werden vernachlässigt.

Unter Verwendung des gleichen Formalismus wie für die Zuggeometrie lassen sich die Nukleationsraten für die unterschiedlichen Partialversetzungen berechnen. Dazu wird im Term für die elastische Arbeit lediglich der Schmidfaktor für die leading und die trailing Partialversetzung getauscht. Man erhält mit diesem Verfahren die Nukleationsraten, wie sie in Abbildung 6.14 gezeigt sind. In der Abbildung wird deutlich, dass zwei Phänomene zum Tragen kommen. Zum einen erfährt die trailing Partialversetzung nun die höhere Schubspannung, da sie einen Schmidfaktor von 0,471 hat. Zum anderen wird die Nukleation zusätzlich begünstigt, da das Auflösen des Stapelfehlers eine Verringerung der Gesamtenergie zur Folge hat. Für die leading Partialversetzung gilt diese Argumentation genau umgekehrt. Durch die kleinere Schubspannung und die Stapelfehlerenergie setzt eine experimentell nachweisbare Nukleationsrate erst bei sehr hohen Spannungen ein. Dies zeigt auch, dass eine Asymmetrie zwischen Zug- und Druckversuch besteht [92].

Für den Fall, dass die trailing Partialversetzung um ein Vielfaches schneller nukleiert wird als die leading Partialversetzung, ist demnach klar, dass im Experiment volle 


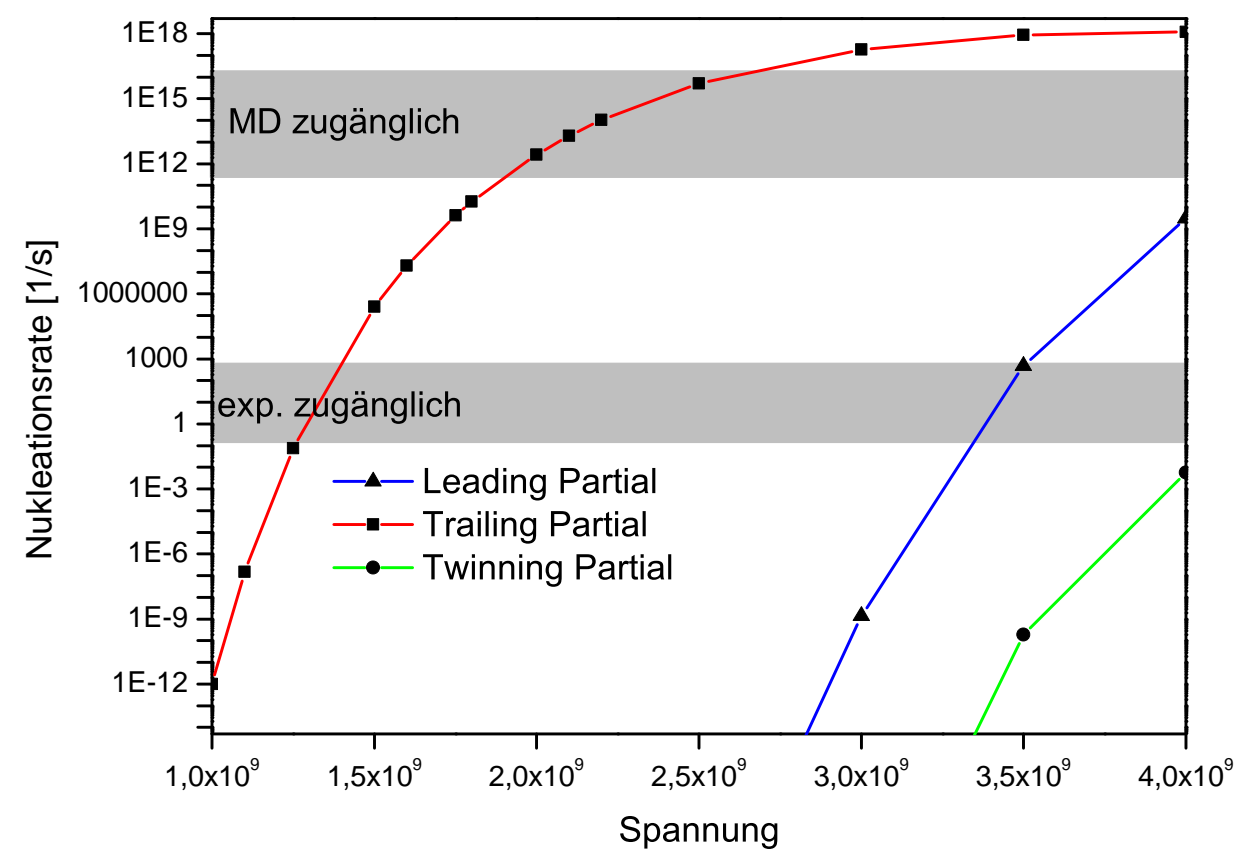

Abbildung 6.14: Nukleationsraten im Druckversuch - Spannungsabhängiger Verlauf der Nukleationsraten für unterschiedliche Versetzungen während eines Druckversuches. 
Versetzungen beobachtet werden müssen. Diese Vorhersage deckt sich mit den experimentellen Befunden für die Biegeversuche.

\subsubsection{Einflüsse der Mikrostruktur auf die Versetzungsbewegung}

In dem zuvor beschriebenen Bild der klassischen Keimbildung wurde erläutert, wie das Auftreten von isolierten Partialversetzungen zu verstehen ist. Demnach wird für einen Zugversuch in [110]-Richtung erwartet, dass die Nukleation einer trailing Partialversetzung nur mit einer sehr viel geringeren Rate auftritt. Dies führt dazu, dass sich im Experiment keine kompakte Versetzung bilden wird. In Abschnitt 5.2 wurde jedoch aufgezeigt, dass in einigen Fällen auch volle Versetzungen in den Drähten beobachtet werden konnten. Die Zusammenfassung dieser Zugversuche lieferte erste bedeutende Hinweise darauf, dass mikrostrukturelle Einflüsse eine mögliche Ursache für das abweichende Verformungsverhalten sein können. Auf diese Einflüsse soll nun in den folgenden Abschnitten genauer eingegangen werden.

Das Auftreten von Extrapunkten wird in der Literatur durch das Vorhandensein unterschiedlicher Defekte im Kristall erklärt. Dabei erweisen sich aus der Liste in Abschnitt 3.2 zwei Defekttypen als besonders beachtenswert für die Versetzungsbewegung: Stapelfehler und Zwillingsgrenzen, die sich parallel zu Drahtachse befinden. Für beide Defekttypen soll im Folgenden abgeschätzt werden inwieweit diese relevant sein können für die Bewegung von Partialversetzungen. Zunächst wird auf die Wechselwirkung einer Partialversetzung mit einem existierenden Stapelfehler eingegangen, als zweites werden dann die möglichen Reaktionen einer Partialversetzung mit einer Zwillingsgrenze aufgezeigt.

\section{Wechselwirkung einer Partialversetzung mit einem Stapelfehler}

Der Fall, dass eine Partialversetzung mit einem existierenden Stapelfehler wechselwirkt trat in vielen Zugversuchen bereits auf: Dadurch, dass bei Zugbelastung in [110]Richtung zwei Gleitsysteme den gleichen Schmidfaktor besitzen, ist es wahrscheinlich, dass sich die Wege der Versetzungen während der Verformung kreuzen. Hier ergibt sich die Frage nach dem Prozess der Wechselwirkung einer Versetzung oder Partialversetzung mit einem existierenden Stapelfehler. Die Wahrscheinlichkeit, dass sich zwei Partialversetzungen direkt im Draht begegnen und wechselwirken, lässt sich bei bekannter Dehnrate relativ einfach abschätzen. Die Bildungsrate für die Defekte konnte 


\section{DISKUSSION}

in dem in Abschnitt 5.1.4 betrachteten Draht ermittelt werden. Sie beträgt 0,22 Defekte pro Sekunde. Dem gegenüber zu stellen ist die Verweildauer einer Versetzung im Draht. Diese beträgt in erster Näherung in einem Draht der Dicke $d$

$$
t_{\text {verweil }}=\frac{d}{v_{\text {disl }}} .
$$

Die Defektgeschwindigkeit wurde schon vorher abgeschätzt und liegt für eine Versetzung in sonst defektfreien Material bei Gold in der Größenordnung von $4 \cdot 10^{2} \mathrm{~m} / \mathrm{s}$ (vergleiche dazu auch Kapitel 4). Eine typische Drahtdicke, bei der Stapelfehler beobachtet wurden, ist $100 \mathrm{~nm}$. Damit kommt für die Verweildauer nur ein Bereich von $10^{-9}$ Sekunden in Betracht. Es ist daher evident, dass bei der geringen Dehnrate die Wahrscheinlichkeit, dass sich zwei Versetzungen begegnen, sehr gering ist. Somit ist der hinreichend aus der Literatur bekannte Fall der Wechselwirkungen zweier Partialversetzungen [93] nicht von großer Relevanz. Auch konnte im Kapitel 5.1 gezeigt werden, dass das Auftreten von Strukturen, die auf ein Treffen von Partialversetzungen deuten nur sehr selten beobachtet wurde. Liegt ein solcher Fall vor, wird erwartet, dass sich ein immobiles Versetzungssegment mit dem Burgersvektor von a/3[100] bildet [94].

Während die Wechselwirkung von Versetzungen mit Zwillingsgrenzen zahlreich beschrieben wurde [95], gibt es bisher kein tiefer gehendes Verständnis der Wechselwirkung von Partialversetzungen mit Stapelfehlern. Einzelne Simulationen zeigen, dass Stapelfehler als Hindernisse für die Bewegung von Partialversetzungen wirken können [96]. Andere Arbeiten gehen davon aus, dass die sich ergebende Struktur beim Durchkreuzen
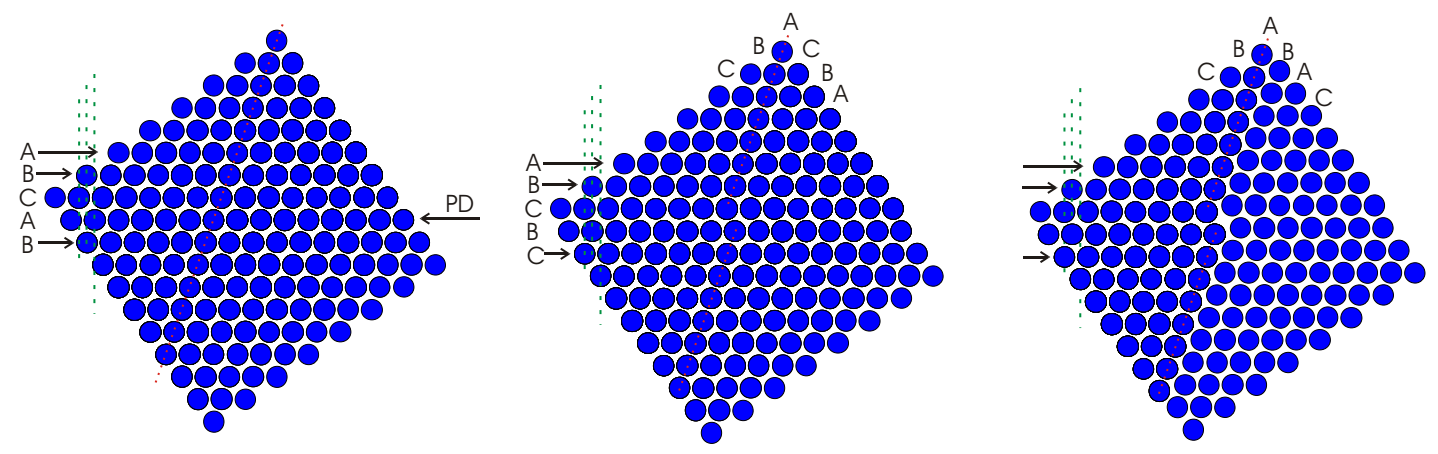

Abbildung 6.15: Atomare Darstellung sich schneidender Stapelfehler - Nach dem Durchlaufen der ersten Partialversetzung entsteht der Stapelfehler, der beim Durchlaufen der zweiten Versetzung geschnitten wird. 
der Stapelfehler einer linienhaften Anhäufung von Gitterleerstellen entspricht [97]. Dieses Bild wird durch einfache Harte-Kugel-Modelle, wie es in Abbildung 6.15 gezeigt ist, gestützt. Im Bereich der sich schneidenden Gleitebenen formt sich ein Extravolumen, dass als Fehlstelle interpretiert werden kann.

Es wird aus den unterschiedlichen Sichtweisen deutlich, dass es noch kein einheitliches atomistisches Bild der Struktur gibt, jedoch trat dieser Fall sehr häufig während der Zugverformung auf. In der Mehrzahl der Fälle konnte gezeigt werden, dass sich eine Partialversetzung nahezu ungehindert durch einen bereits existierenden Stapelfehler bewegen kann. Ein typische Aufnahme dieses Durchkreuzens ist in Abbildung 5.20 gezeigt.

\section{Wechselwirkungen von Versetzungen mit Zwillingen}

Die Wechselwirkung einer Versetzung mit einer Zwillingsgrenze ist im Vergleich zu der vorher beschriebenen Interaktion in der Literatur hinreichend bekannt [95]. Während eine Schraubenversetzung ohne Dissoziation einfach in ein Gleitsystem des Zwillings wechseln und damit den kompletten Draht durchlaufen kann, ist für eine Partialversetzung die Situation komplizierter. Eine systematische Untersuchung aller bisher bekannten Mechanismen ist in [95] gegeben. Im Allgemeinen führt die Wechselwirkung einer Partialversetzung mit der Zwillingsgrenze dazu, dass die Partialversetzung einen sesshaften Teil an der Zwillingsgrenze deponiert. Dieser Prozess ist allerdings mit einer erhöhten Energiebarriere verknüpft. Wird ein nicht sesshafter Teil durch die Dissoziation erzeugt, liegt dieser in der Ebene der Zwillingsgrenze. Die Bewegung dieser Versetzung führt dazu, dass die Zwillingsgrenze um eine $\{111\}$-Ebene parallel verschoben wird. Dies setzt allerdings voraus, dass auf die mobile Partialversetzung auch eine Schubspannung wirkt. Bei den hier auftretenden herstellungsbedingten Zwillingen ist dies allerdings nicht der Fall, da diese parallel zur Drahtachse liegen. Damit ist die Zwillingsgrenze eine Ebene, die keine Schubspannung spürt, Versetzungselemente sollten somit auch in diesem Fall ortsfest bleiben.

Diese einfachen Überlegungen zeigen, dass es mit zunehmender Verformung aufgrund der Einschränkungen der Bewegung der Versetzungen zu einem Aufstauen im Draht kommen muss [98]. Eine weitere Form der Wechselwirkung wurde in MD Simulationen beobachtet. Dort trat der Fall auf, dass eine Partialversetzung an einer Zwillingsgrenze 


\section{DISKUSSION}

in zwei Teile dissoziiert. Ein Teil verbleibt als immobile Partialversetzung an der Grenze, der andere Teil läuft als volle Versetzung im verzwillingten Bereich weiter. [87, 99]. In der Summe kann man folgern, dass eine Zwillingsgrenze ein sehr viel stärkeres Hindernis für die Versetzungsbewegung darstellt als ein Stapelfehler [100]. Die Tatsache, dass die Wechselwirkung stets mit einer Energiebarriere verknüpft ist und als Reaktionsprodukt das Auftreten von vollen Versetzungen beinhaltet, lässt darauf schließen, dass dies das Auftreten von vollen Versetzungen erklärt.

\subsubsection{Kombination der vorliegenden Modelle}

In den vorangegangenen Abschnitten wurden zwei Konzepte erläutert, das Vorhersagen von Verformungsmechanismen zu erklären. Beide Konzepte gehen dabei von zwei grundsätzlich unterschiedliche Ansätze aus. Im Fall der klassischen Nukleationstheorie wurden stabile Keimgrößen aufgrund von Energiebetrachtungen berechnet. Die Versetzung wurde als voll ausgebildeter Teil des Keims angesehen und hat stets einen festen Burgersvektor. Im Falle des GSFE Konzepts spielte vielmehr der variable Energieverlauf bei kontinuierlicher Verschiebung eine fundamentale Rolle. Hier waren nicht die Keimgrößen entscheidend für den Defekttyp, sondern der materialspezifische Verlauf der Stapelfehlerenergien.

Derzeit werden in der Literatur Versuche unternommen beide Ideen in einem Konzept zu vereinen. Dabei wird zum Beispiel der Stapelfehlerenergie eine Größe, die abhängig ist von der Verschiebung, und fließt als Wert in die Energiebilanz eines Versetzungskeims mit ein. Es stellt sich bei den Berechnungen allerdings heraus, dass das Bild der GSFE dabei nicht so statisch ist, wie von [65] angenommen, sondern selbst auch eine Funktion der angelegten Spannung wird [101]. Dieser Umstand ist in Abbildung 6.16 gezeigt. Dort wurde berechnet, wie sich der Verlauf der GSFE verändert, wenn die Kristallhälften nicht starr gegeneinander verschoben werden, sondern durch eine Scherspannung verformt werden. Das Resultat ist eine Absenkung der Kurve für die Stapelfehlerenergie. Die Beschreibung der aufkeimenden Verformung wird damit eine Funktion mehrerer Variablen. Inwieweit diese erweiterte Beschreibung der Keimbildung in der Zukunft weiter verfolgt wird, ist derzeit noch nicht abzuschätzen. Die einfache Anwendung auf die in dieser Arbeit gewonnenen experimentellen Ergebnisse hingegen ist somit nicht möglich. Vor dem Hintergrund der relativ guten Fähigkeit beider Modelle, die Ergebnisse wieder zu geben, und der Komplexität der nötigen Erweiterungen 

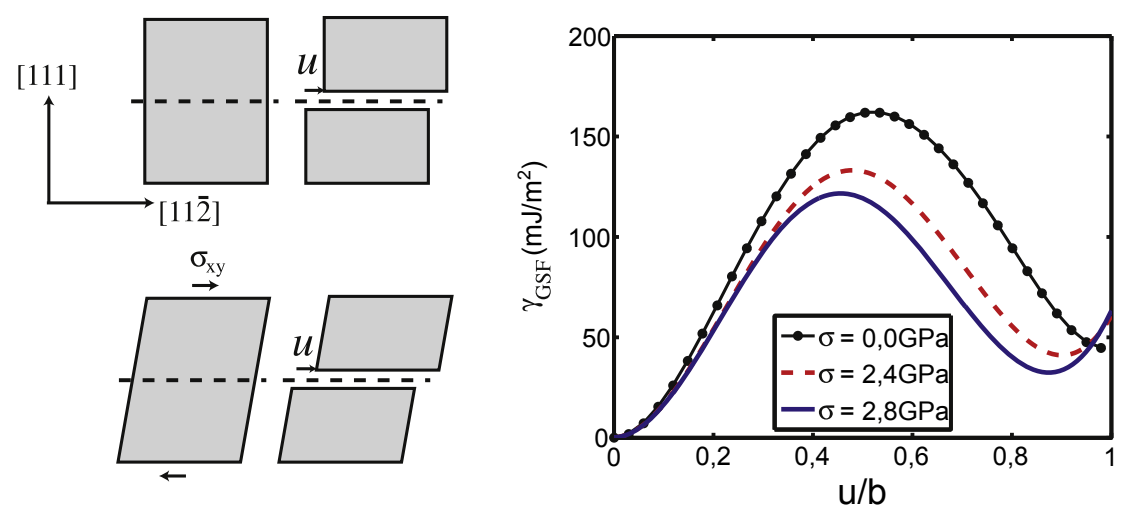

Abbildung 6.16: Spannungsabhängiger Verlauf der GSFE - Durch die Betrachtung der Verschiebung unter Scherspannung ergeben sich Unterschiede im Verlauf der GSFE. Dies führt zu einer komplexeren Beschreibung der Nukleationsproblematik. (aus [101])

der vorhandenen Modelle wird an dieser Stelle darauf verzichtet, dieses Konzept weiter zu verfolgen.

Vergegenwärtigt man sich am Ende dieses Kapitels noch einmal die Kernaussage, erscheint ein Aspekt besonders interessant: Im Modell der klassischen Keimbildung wird davon ausgegangen, dass die Nukleation einer Versetzung vorbestimmt ist durch die Beschaffenheit der Oberfläche. Es konnte gezeigt werden, dass eine existierende Stufe eine wesentliche Rolle für die Verformung spielt. Vergleicht man diese Tatsache mit den frühen Ergebnissen von Brenner [1], wie sie in der Einleitung herangezogen wurden, stellt man fest, dass die Vermutungen seiner Arbeit auf ein neues Fundament gestellt werden konnten. 
6. DISKUSSION 


\section{7}

\section{Fazit und Ausblick}

Die vorangegangenen Kapitel dieser Arbeit haben die zentralen Ergebnisse dieser Arbeit detailiert dargestellt. Es konnten dabei auf zwei unterschiedlichen Ebenen Neuerungen und Weiterentwicklungen erarbeitet werden. Zum einen wurde gezeigt, dass mit Hilfe einer geeigneten Rahmenstruktur eine kontrollierte Verformung von 1-dimensionalen Strukturen im TEM möglich ist. Die Steifigkeit dieses Aufbaus in Verbindung mit den gewählten Proben ließen eine kontrollierte Verformung der Proben zu. Dies hat einen entscheidenden Einfluss auf den beobachtbaren Verformungsmechanismus [27]. Zum anderen konnten die so gewonnenen Ergebnisse mit Hilfe eines Modells in sehr guter Übereinstimmung erklärt werden. Dazu sind hinlänglich bekannte Konzepte für die klassische Versetzungsnukleation in einer ganzheitlichen Beschreibung zusammengeführt worden. In diesem Bild ist der beobachtete Unterschied im Verformungsverhalten zwischen makroskopischen Proben und Proben im Nanometerbereich kein direkter Größeneffekt. In der verwendeten Beschreibung ist keine Längenskala enthalten, die einen Übergang im Verformungsverhalten bei größeren Proben nötig machen würde. Messungen der Streckgrenze an diesen Drähten unterstützen diese Vorhersage [24]. In dem in dieser Arbeit abgedeckten Größenbereich ist kein signifikanter Anstieg in der Festigkeit der Proben zu erkennen. Demnach sollten auch für perfekte, makroskopische Proben Partialversetzungen die Verformung tragen. Im Experiment wird dies allerdings nur schwer realisierbar sein, da mit zunehmendem Probenvolumen auch die Wahrscheinlichkeit eines vorhandenen Defektes wächst. Dass die vorhandenen Defekte den Verformungsmodus erheblich beeinflussen können, konnte ebenfalls in dieser Arbeit aufgezeigt werden. 


\section{FAZIT UND AUSBLICK}

In dem entwickelten Modell sind aber nicht nur der Defekttyp leicht darstellbar, sondern es eröffnen sich darüber hinaus weitergehende Möglichkeiten der Interpretation. Auf diese Weise ist mit der Beschreibung erstmals der direkte Vergleich von realem Experiment und einer MD Simulation zugänglich. So kann eine Verbindung zwischen den Ergebnissen dieser Herangehensweisen hergestellt werden.

Als weiterführende Untersuchungen bieten sich zwei Richtungen an. Einerseits gibt es noch einige Aspekte, deren Untersuchung das Modell direkt stark stützen würden. Zum Beispiel war ein wesentlicher Punkt die Existenz einer hinreichend großer Zahl an Oberflächenstufen. Diese konnten in den Zugaufbauten nicht beobachtet werden, da die Oberfläche mit einer Platinschicht bedeckt war. Dennoch sollte es möglich sein, an geeignet präparierten Proben mit Hilfe von hochauflösender Mikroskopie diese Stufen nachzuweisen und auch zu quantifizieren. An verformten Proben könnten die Nukleationspunkte der Stapelfehler ebenfalls auf diese Oberflächenmerkmale hin untersucht werden. Eine gezielte Untersuchung des Einflusses einer imperfekten Ausrichtung der Drähte zur Zugachse wird derzeit angegangen. Ebenso hat sich in quantitativen Zugversuchen im REM [102] gezeigt, dass eine Dehnratenabhängigkeit der Fließspannung existiert. Diese kann im Modell der klassischen Nukleationstheorie bisher nicht erklärt werden. Daher ist an dieser Stelle eine Erweiterung des Vorhandenen vonnöten.

Die andere Richtung hat eine grundlegende Bedeutung für das Verständnis der Verformung in Metallen. Die Tatsache, dass die Ergebnisse dieser Arbeit im entwickelten Modell ohne Weiteres darstellbar sind, ermöglicht eine neue Betrachtungsweise für viele weitere Fragestellungen. Einige sich sofort ergebende Punkte sollen dies exemplarisch bekräftigen

- Mit Hilfe eines Biegeversuches konnte der Verformungsmechanismus verändert werden. Dies ist nach dem Modell zu erwarten und geht einher mit einer Asymmetrie in der Fließspannung. Ein quantitativer Nachweis an den verwendeten Proben steht noch aus.

- Ein wesentlicher Parameter in dem verwendeten elastischen Modell der Versetzung stellt die Stapelfehlerenergie dar. Daher ist eine Untersuchung von Materialien mit stark von dem Wert für Gold abweichender Stapelfehlerenergie lohnenswert. Der Vergleich der Ergebnisse aus einem solchen Experiment mit dem rechnerischen Ergebnis wäre eine lohnenswerte Aufgabe. 
- Das Verformungsverhalten könnte untersucht werden, wenn das Modell an eine andere Versetzungsquelle als die Oberfläche (zum Beispiel eine Frank-ReadQuelle) angepasst werden würde. Damit wäre ein direkter Vergleich zwischen defektfreien Proben und Proben, deren Verformung durch Keimbildung limitiert ist, möglich.

Aus den oben aufgeführten Möglichkeiten wird deutlich, dass durch die Einführung eines neuen Modells im Rahmen dieser Arbeit ein geeigneter Ausgangspunkt für weitergehende Untersuchungen gelegt werden konnte. 


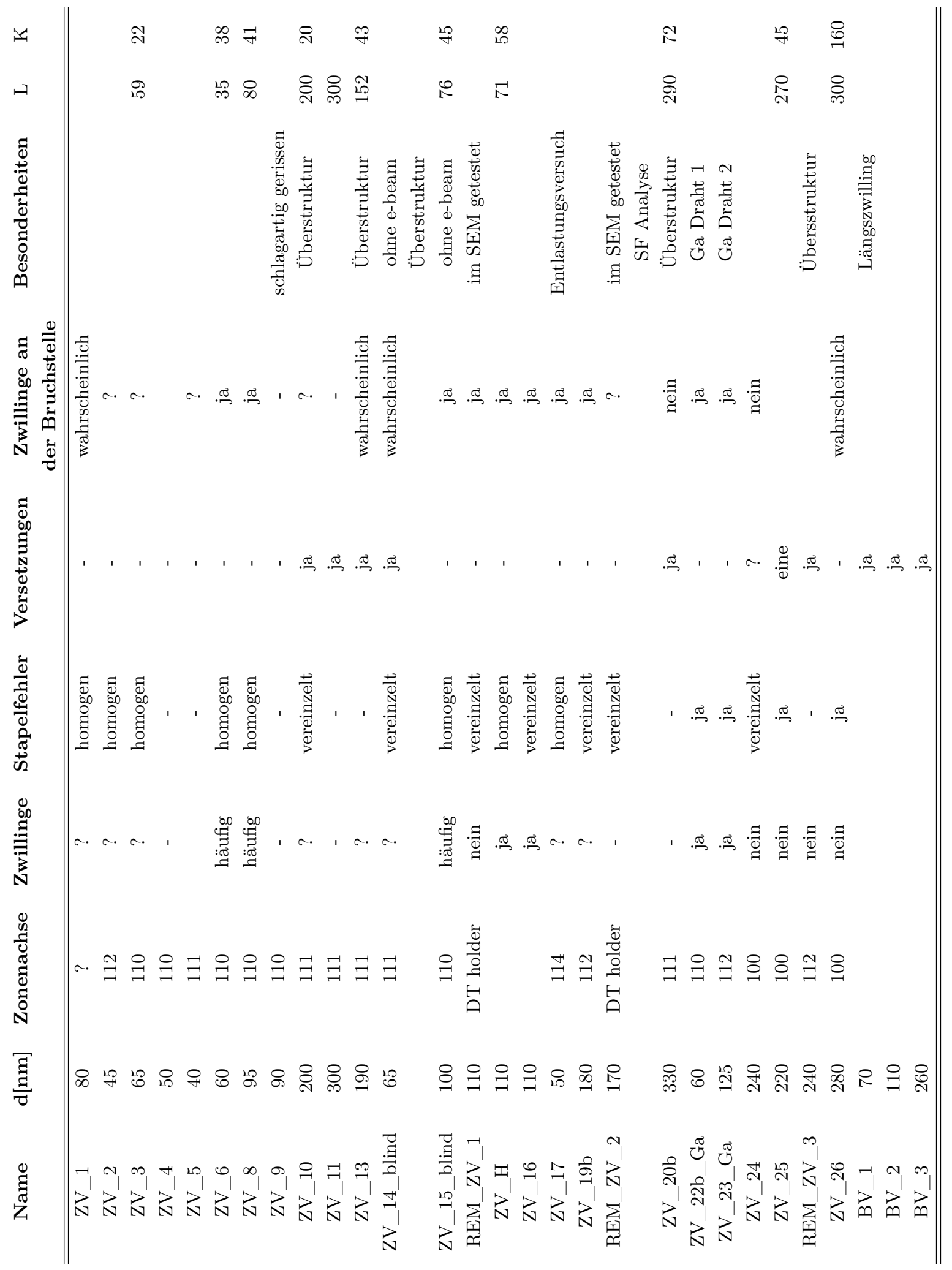




\section{Literatur}

[1] S. S. Brenner. Tensile Strength of Whiskers. Journal of Applied Physics, 27(12):1484-1491, 1956. 1, 2, 93, 155

[2] J. D. Eshelby, F. C. Frank, and F. R. N. Nabarro. The equilibrium of linear arrays of dislocations. Philosophical Magazine Series 7, 42(327):351364, 1951. 1

[3] E. O. Hall. The Deformation and Ageing of Mild Steel: III Discussion of Results. Proceedings of the Physical Society. Section B, 64(9):747-753, 1951. 1

[4] A.H. Cottrell. Dislocations and plastic flow in crystals. Clarendon Press, 1953. 1

[5] E.T. Lilleodden And W.D. Nix. Microstructural length-scale effects in the nanoindentation behavior of thin gold films. Acta Materialia, 54(6):1583-1593, 2006. 3

[6] S. S. Brenner. Strength of gold whiskers. Journal of Applied Physics, 30(2):266-267, 1959. 3

[7] Julia R. Greer and William D. Nix. Nanoscale gold pillars strengthened through dislocation starvation. Physical Review B, 73(24):245410, 2006. 3, 4, 51, 93

[8] D. Kiener And A.M. Minor. Source-controlled yield and hardening of $\mathbf{C u}(100)$ studied by in situ transmission electron microscopy. Acta Materialia, 59(4):1328-1337, February 2011. 4

[9] E.A. Withey, A.M. Minor, D.C. Chrzan, J.W. Morris Jr., and S. KuRAмото. The deformation of Gum Metal through in situ compression of nanopillars. Acta Materialia, 58(7):2652-2665, April 2010. 4 
[10] S.H. Oh, M. Legros, D. Kiener, P. Gruber, and G. Dehm. In situ TEM straining of single crystal Au films on polyimide: Change of deformation mechanisms at the nanoscale. Acta Materialia, 55(16):5558-5571, September 2007. 4, 5, 116, 118

[11] G. Dehm, S.H. Oh, P. Gruber, M. Legros, and F.D. Fischer. Strain compensation by twinning in Au thin films: Experiment and model. Acta Materialia, 55(19):6659-6665, November 2007. 5

[12] G. Dehm, T.J. Balk B. J. Inkson, T. Wagner, and E. Arzt. Plasticity and Interfacial Dislocation Mechanisms in Epitaxial and Polycrystalline Al Films Constrained by Substrates. Journal Materials Science and Technology, 18:113-117, 2002. 5

[13] Marc Legros, Martiane Cabie, and Daniel S. Gianola. In situ deformation of thin films on substrates. Microscopy Research and Technique, 72(3):270-283, March 2009. 5

[14] R. Dou And B. Derby. Deformation mechanisms in gold nanowires and nanoporous gold. Philosophical Magazine, 91(7):1070-1083, 2011. 5

[15] Eike Epler. Mechanisches Verhalten von nanoporösem Gold und Kompositen. Diplomarbeit, Universität Göttingen, 2011. 5

[16] Tokushi Kizuka. Atomistic visualization of deformation in gold. Phys. Rev. B, 57(18):11158-11163, May 1998. 6

[17] Yang Lu, Jun Song, Jian Huang, and Jun Lou. Surface dislocation nucleation mediated deformation and ultrahigh strength in sub-10-nm gold nanowires. Nano Research, pages 1-7. 6

[18] Harold S. Park, Ken Gall, and Jonathan A. Zimmerman. Deformation of FCC nanowires by twinning and slip. Journal of the Mechanics and Physics of Solids, 54(9):1862-1881, September 2006. 7, 111, 122

[19] K. Gall, J. Diao, and M.L. Dunn. The Strength of Gold Nanowires. Nano Letters, 4(12):2431-2436, 2004. 7

[20] Jiankuai Diao, Ken Gall, Martin L. Dunn, and Jonathan A. ZimmerMAN. Atomistic simulations of the yielding of gold nanowires. Acta Materialia, 54(3):643-653, February 2006. 7 
[21] H. Van Swygenhoven, P.M. Derlet, and A.G. FrÃ seth. Nucleation and propagation of dislocations in nanocrystalline fcc metals. Acta Materialia, 54(7):1975-1983, 2006. 8

[22] Vesselin Yamakov, Dieter Wolf, Simon R. Phillpot, Amiya K. MukHerJeE, AND HeRbert Gleiter. Dislocation processes in the deformation of nanocrystalline aluminium by molecular-dynamics simulation. Nat Mater, 1(1):45-49, September 2002. 8

[23] K. S. Kumar, H. Van Swygenhoven, and S. Suresh. Mechanical behavior of nanocrystalline metals and alloys. Acta Materialia, 51(19):5743-5774, 2003. The Golden Jubilee Issue. Selected topics in Materials Science and Engineering: Past, Present and Future. 8

[24] Bahne KAPELle. In situ Zugversuche an einkristallinen Gold Nanodrähte im REM. Diplomarbeit, Universität Göttingen, 2011. 9, 10, 35, 49, 51, 81, 90, 121, 141, 157

[25] Jong-Hyun Seo, Youngdong Yoo, Na-Young Park, Sang-Won Yoon, Hyoban Leev Sol Han, Seok-Woo Lee, Tae-Yeon Seong, Seung-Cheol Lee, Kon-Bae lee, Pil-Ryung Cha, Harold S. Park, Bongsoo Kim, and Jae-Pyoung Ahn. Superplastic Deformation of Defect-Free Au Nanowires via Coherent Twin Propagation. Nano Letters, 11:3499-3502, 2011. 9, 111, 142

[26] Andreas Sedlmayr, Erik Bitzek, Daniel S. Gianola, Gunther Richter, Reiner Mönig, and Oliver Kraft. Existence of Two TwinningMediated Plastic Deformation Modes in Au Nanowhiskers. (submitted), 2012. 10,142

[27] B. Kapelle, B. Roos, G. Richter, and C.A. Volkert. In situ tensile test of single crystal metal nanowires inside a scanning electron microscope. Review of Scientific Instruments, noch nicht eingereicht. 10, 143, 157

[28] C. B. Carter and D B. Williams. Transmission Electron Microscopy. Plenum Press , New York, 1996. 11, 79

[29] G. Gottstein. Physikalische Grundlagen der Materialkunde. Springer, 3rd edition edition, 2007. 13 
[30] N. Bernstein and E. B. Tadmor. Tight-binding calculations of stacking energies and twinnability in fcc metals. Physical Review B, 69(9):094116, March 2004. 15, 128, 134

[31] T. S. BYUn. On the stress dependence of partial dislocation separation and deformation microstructure in austenitic stainless steels. Acta Materialia, 51(11):3063-3071, June 2003. 15, 117, 122

[32] J. W. Christian and S. Mahajan. Deformation twinning. Progress in Materials Science, 39(1-2):1-157, 1995. 18

[33] J. A. Venables. Deformation twinning in face-centred cubic metals. Philosophical Magazine, 6(63):379-396, March 1961. 18

[34] G. Richter, K. Hillerich, D. S. Gianola, R. Monig, O. Kraft, and C. A. Volkert. Ultrahigh Strength Single Crystalline Nanowhiskers Grown by Physical Vapor Deposition. Nano Letters, 9(8):3048-3052, August 2009. 33, 35, 93

[35] P B Hirsch, A Kelly, and J W Menter. The Structure of Cold Worked Gold I: A Study by Electron Diffraction. Proceedings of the Physical Society, 68:1132-1145, 1955. 38

[36] D. W. Pashley and M. J. Stowell. Electron microscopy and diffraction of twinned structures in evaporated films of gold. Philosophical Magazine, 8(94):1605-1632, October 1963. 38

[37] D. Cherns. Direct resolution of surface atomic steps by transmission electron microscopy. Philosophical Magazine, 30(3):549-556, September 1974. 38

[38] J.C. Heyraud and J.J. Metois. Anomalous 422 diffraction spots from \{111\} flat gold crystallites: (111) surface reconstruction and moire fringes between the surface and the bulk. Surface Science, 100:519-528, 1980. 38

[39] V. Germain, Jing Li, D. Ingert, Z. L. Wang, and M. P. Pileni. Stacking Faults in Formation of Silver Nanodisks. The Journal of Physical Chemistry $B, \mathbf{1 0 7}(34): 8717-8720$, August 2003. 39

[40] CuBe-Rahmen. Institut für Materialforschung II. Forschungszentrum Karlsruhe. 42 
[41] Kodanda Ram Mangipudi. Modellierung der Rahmenstruktur und FE Rechnungen zu der Verformung. (unveröffentlichte Arbeit). 46, 47

[42] J. J. Gilman. Dislocation Mobility in Crystals. Journal of Applied Physics, 36:3195, 1965. 51

[43] I. Utke, V. Friedli, S. Fahlbusch, S. Hoffmann, P. Hoffmann, and J. Michler. Tensile Strengths of Metal-Containing Joints Fabricated by Focused Electron Beam Induced Deposition. Advanced Engineering Materials, 8(3):155-157, 2006. 78

[44] G. Dehm, T. J. Balk, H. Edongue, and E. Arzt. Small-scale plasticity in thin $\mathbf{C u}$ and Al films. Microelectronic Engineering, 70(2-4):412-424, November 2003. 84

[45] H. Conrad and K. Jung. Effect of grain size from $\mathbf{m m}$ to $\mathbf{~ n m}$ on the flow stress and plastic deformation kinetics of $\mathrm{Au}$ at low homologous temperatures. Materials Science and Engineering: A, 406(1-2):78-85, October 2005. 91

[46] N. Agrait, G. Rubio, and S. Vieira. Plastic deformation of nanometerscale gold connective necks. Physical Review Letters, 74:3995-3999, 1995. 91

[47] Bin Wu, Andreas Heidelberg, and John J. Boland. Mechanical properties of ultrahigh-strength gold nanowires. Nature Materials, 4(7):525-529, July 2005. 91

[48] V. Vidal, L. Thilly, F. Lecouturier, and P.-O. Renault. Effects of size and geometry on the plasticity of high-strength copper/tantalum nanofilamentary conductors obtained by severe plastic deformation. Acta Materialia, 54(4):1063-1075, February 2006. 92

[49] Z. W. Shan, Raja K. Mishra, S. A. Syed Asif, Oden L. Warren, and AnDREW M. MinOR. Mechanical annealing and source-limited deformation in submicrometre-diameter Ni crystals. Nature Materials, 7(2):115-119, February 2008. 92

[50] D. Kiener And A. M. Minor. Source Truncation and Exhaustion: Insights from Quantitative in situ TEM Tensile Testing. Nano Lett., 11(9):3816-3820, July 2011. 92 
[51] Hai-Jun Jin, Lilia Kurmanaeva, Jörg Schmauch, Harald Rösner, Yulia IVanisenko, and Jörg Weissmüller. Deforming nanoporous metal: Role of lattice coherency. Acta Materialia, 57(9):2665-2672, May 2009. 92

[52] Sang Ho Oh, Marc Legros, Daniel Kiener, and Gerhard Dehm. In situ observation of dislocation nucleation and escape in a submicrometre aluminium single crystal. Nature Materials, 8(2):95-100, February 2009. 93, 122

[53] C. A. Volkert and A. M. Minor. Focused Ion Beam Microscopy and Micromachining. MRS Bulletin, 32(5):389-399, May 2007. 93

[54] D. Häussler, M. Bartsch, U. Messerschmidt, and B. Reppich. HVTEM in situ observations of dislocation motion in the oxide dispersion strengthened superalloy MA 754. Acta Materialia, 49(18):3647-3657, October 2001. 93

[55] James F. Ziegler, M.D. Ziegler, and J.P. Biersack. SRIM - The stopping and range of ions in matter (2010). Nuclear Instruments and Methods in Physics Research Section B: Beam Interactions with Materials and Atoms, 268(11-12):1818-1823, June 2010. 95

[56] D. Kiener, C. Motz, M. Rester, M. Jenko, and G. Dehm. FIB damage of $\mathrm{Cu}$ and possible consequences for miniaturized mechanical tests. Materials Science and Engineering: A, 459(1-2):262-272, June 2007. 99

[57] Cynthia L. Kelchner, S. J. Plimpton, and J. C. Hamilton. Dislocation nucleation and defect structure during surface indentation. Physical Review B, 58(17):11085-11088, November 1998. 116

[58] William M Haynes. Handbook of Chemistry and Physics. CRC Press, 91 edition, 2010. 118

[59] V. Yamakov, D. Wolf, S. R. Phillpot, A. K. Mukherjee, and H. GleiTER. Deformation-mechanism map for nanocrystalline metals by molecular-dynamics simulation. Nature Materials, 3(1):43-47, January 2004. 121

[60] D. Kiener, P. Hosemann, S. A. Maloy, and A. M. Minor. In situ nanocompression testing of irradiated copper. Nature Materials, 10(8):608-613, August 2011. 122 
[61] Christopher R. Weinberger, Andrew T. Jennings, Keonwook Kang, and Julia R. Greer. Atomistic simulations and continuum modeling of dislocation nucleation and strength in gold nanowires. Journal of the Mechanics and Physics of Solids, 60(1):84 - 103, 2012. 123, 127, 128, 129

[62] Changuiang Ji and Harold S Park. The coupled effects of geometry and surface orientation on the mechanical properties of metal nanowires. Nanotechnology, 18(30):305704, 2007. 123

[63] Mingwei Chen, En Ma, Kevin J. Hemker, Hongwei Sheng, Yinmin Wang, and Xuemei Cheng. Deformation Twinning in Nanocrystalline Aluminum. Science, 300(5623):1275-1277, May 2003. 123, 130

[64] Wuwei Liang and Min Zhou. Atomistic simulations reveal shape memory of fcc metal nanowires. Phys. Rev. B, 73(11):115409, March 2006. 123

[65] V. ViTEK. Intrinsic stacking faults in body-centred cubic crystals. Philosophical Magazine, 18(154):773-786, October 1968. 124, 125, 154

[66] James R. RICE. Dislocation nucleation from a crack tip: An analysis based on the Peierls concept. Journal of the Mechanics and Physics of Solids, 40(2):239-271, 1992. 124, 126

[67] E.B. Tadmor and S. Hai. A Peierls criterion for the onset of deformation twinning at a crack tip. Journal of the Mechanics and Physics of Solids, 51(5):765-793, May 2003. 126, 127

[68] S. Hai and E. B. Tadmor. Deformation Twinning at Aluminum Crack Tips. Acta Materialia, 51:117, 2003. 126

[69] B.Q. Li, M.L. Sui, And S.X. Mao. Twinnability Predication for fcc Metals. Journal of Materials Sciences and Technology, 27:97-100, 2011. 126

[70] Y. Liu, D. Bufford, H. Wang, C. Sun, and X. Zhang. Mechanical properties of highly textured Cu/Ni multilayers. Acta Materialia, 59(5):19241933, March 2011. 127

[71] John Price Hirth and Jens Lothe. Theory of Dislocations. John Wiley \& Sons, 2. edition, 1982. 131, 133, 138 
[72] P. Hirel, J. Godet, S. Brochard, L. Pizzagalli, and P. Beauchamp. Determination of activation parameters for dislocation formation from a surface in fcc metals by atomistic simulations. Phys. Rev. B, 78(6):064109, August 2008. 131, 132, 133

[73] J.P HIRTH. The influence of surface structure on dislocation nucleation. The Relation between Structure 85 Mechanical Properties of Metals, page 218, 1963. 131

[74] G. E. Beltz and L. B. Freund. On the Nucleation of Dislocations at a Crystal Surface. physica status solidi (b), 180(2):303-313, 1993. 133

[75] S. Brochard, P. Beauchamp, and J. Grilhe. Energies of the atomic steps formed on low-index surfaces of fcc metals. Philosophical Magazine Letters, 77(3):125-134, March 1998. 133

[76] F Appel and U Messerschmidt. Surface-Induced Cross Glide of Screw Dislocations. Physica status solidi (b), 35:1003-1008, 1969. 133

[77] Guanshui Xu and Chuanli Zhang. Analysis of dislocation nucleation from a crystal surface based on the Peierls-Nabarro dislocation model. Journal of the Mechanics and Physics of Solids, 51(8):1371-1394, August 2003. 138

[78] Robert J. Asaro and Subra Suresh. Mechanistic models for the activation volume and rate sensitivity in metals with nanocrystalline grains and nano-scale twins. Acta Materialia, 53(12):3369-3382, July 2005. 138

[79] W.N. Li, H.L. Duan, K. Albe, and J. Weissmüller. Line stress of step edges at crystal surfaces. Surface Science, 605(9-10):947-957, May 2011. 141

[80] Z. L. Wang, R. P. Gao, B. Nikoobakht, and M. A. El-Sayed. Surface reconstruction of the unstable 110 surface in gold nanorods. Journal of Physical Chemistry B, 104(23):5417-5420, June 2000. 142

[81] Yang Lu, Jun Song, Jian Yu Huang, and Jun Lou. Fracture of Sub-20nm Ultrathin Gold Nanowires. Advanced Functional Materials, 21(20):3982-3989, 2011. 142

[82] Chuang Deng and Frederic Sansoz. Effects of twin and surface facet on strain-rate sensitivity of gold nanowires at different temperatures. Physical Review B, 81(15):155430, April 2010. 142 
[83] L. Pastor-Abia, M. J. Caturla, E. Sanfabián, G. Chiappe, and E. Louis. Abnormal stress drop at the yield point of aluminum nanowires: A molecular dynamics study. Physical Review B, 83(16):165441, April 2011. 143

[84] Jiankuai Diao, Ken Gall, and Martin L. Dunn. Surface-stress-induced phase transformation in metal nanowires. Nature Materials, 2(10):656-660, October 2003. 143

[85] E. Rabkin, H.-S. Nam, and D.J. Srolovitz. Atomistic simulation of the deformation of gold nanopillars. Acta Materialia, 55(6):2085-2099, April 2007. 143

[86] T. J. BAlk and K. J. Hemker. High resolution transmission electron microscopy of dislocation core dissociations in gold and iridium. Philosophical Magazine A, 81(6):1507-1531, 2001. 144

[87] C. Deng and F. Sansoz. Fundamental differences in the plasticity of periodically twinned nanowires in $\mathrm{Au}, \mathrm{Ag}, \mathrm{Al}, \mathrm{Cu}, \mathrm{Pb}$ and $\mathrm{Ni}$. Acta Materialia, 57(20):6090-6101, December 2009. 147, 154

[88] Zhiming Chen, Zhaohui Jin, and Huajian Gao. Repulsive force between screw dislocation and coherent twin boundary in aluminum and copper. Phys. Rev. B, 75(21):212104-, June 2007. 147

[89] Sandrine Brochard, Pierre Beauchamp, and Jean Grilhe. Simulations of dislocation nucleation from atomic size surface steps and grooves. Materials Science and Engineering A, 309-310:456-462, 2001. 148

[90] Frederic Sansoz, Hanchen Huang, and Derek Warner. An atomistic perspective on twinning phenomena in nano-enhanced fcc metals. JOM Journal of the Minerals, Metals and Materials Society, 60(9):79-84, 2008. 10.1007/s11837-008-0124-x. 148

[91] Toshio Seki, Takaaki Aoki, Jiro Matsuo, and Isao Yamada. STM observation of surface vacancies created by ion impact. Nuclear Instruments and Methods in Physics Research Section B: Beam Interactions with Materials and Atoms, 164-165(0):650-655, April 2000. 148

[92] M A Tschopp, D E Spearot, and D L McDowell. Atomistic simulations of homogeneous dislocation nucleation in single crystal copper. 


\section{LITERATUR}

Modelling and Simulation in Materials Science and Engineering, 15(7):693-709, 2007. 149

[93] E. Carrasco, O. R. De La Fuente, and J. M. Rojo. Dislocation emission at the onset of plasticity during nanoindentation in gold. Philosophical Magazine, 88(3):281-296, 2008. Carrasco, E. Rodriguez De La Fuente, O. Rojo, J. M. 152

[94] Y. C. Lin and D. J. Pen. Atomistic behavior analysis of Cu nanowire under uniaxial tension with maximum local stress method. Molecular Simulation, 33(12):979-988, 2007. 152

[95] Y.T. Zhu, X.L. Wu, X.Z. Liao, J. Narayan, L.J. Kecskes, and S.N. MatHAUDHU. Dislocation-twin interactions in nanocrystalline fcc metals. Acta Materialia, 59:812-821, 2011. 152, 153

[96] Kedarnath Kolluri, M. Rauf Gungor, and Dimitrios Maroudas. Molecular-dynamics simulations of stacking-fault-induced dislocation annihilation in prestrained ultrathin single-crystalline copper films. $J$. Appl. Phys., 105(9):093515-8, May 2009. 152

[97] Benjamin W. van de WaAl. Cross-twinning model of fcc crystal growth. Journal of Crystal Growth, 158:153-165, 1996. 153

[98] M. Chassagne, M. Legros, and D. Rodney. Atomic-scale simulation of screw dislocation/coherent twin boundary interaction in $\mathrm{Al}, \mathrm{Au}, \mathrm{Cu}$ and Ni. Acta Materialia, 59(4):1456-1463, February 2011. 153

[99] Frederic Sansoz and Kevin D. Stevenson. Relationship between hardness and dislocation processes in a nanocrystalline metal at the atomic scale. Physical Review B, 83(22):224101, June 2011. 154

[100] E. Bitzek. Atomistic Study of Twinning in Gold Nanowhiskers. Journal of Solid Mechanics and Materials Engineering, 6:99-105, 2012. 154

[101] Sylvie Aubry, Keonwook Kang, Seunghwa Ryu, and Wei Cai. Energy barrier for homogeneous dislocation nucleation: Comparing atomistic and continuum models. Scripta Materialia, 64:1043-1046, 2011. 154, 155

[102] Bahne Kapelle. Dehnratenabhängigkeit der Fließspannung von Au Nanodrähten. (persönliche Mitteilung). 158 


\section{Eidesstattliche Erklärung}

Ich erkläre hiermit an Eides statt, dass ich die vorliegende Arbeit mit dem Titel "In-situ Zugversuche an einkristallinen Gold-Nanodrähten im TEM" ohne unzulässige Hilfe Dritter und ohne Benutzung anderer als der angegebenen Hilfsmittel angefertigt habe.

Göttingen, 
LITERATUR 


\section{Danksagung}

An dieser Stelle möchte ich den vielen Personen danken, die mich während dieses Lebensabschnittes begleitet haben.

Zuerst geht mein Dank an Prof. Cynthia Volkert, die mir die Gelegenheit gegeben hat diese Arbeit in ihrer Arbeitsgruppe durchzuführen. Ihre stete Diskussionsbereitschaft und Anregungen haben wesentlich Einfluss auf diese Arbeit genommen.

Prof. Dr. Michael Seibt möchte ich für die Übernahme des Korreferats danken. Darüber hinaus durfte ich dankenswerterweise einige Male auf sein Fachwissen auf dem Gebiet der Elektronenmikroskopie zurückgreifen.

Dr. Gunther Richter danke ich für die Bereitstellung des verwendeten Probenmaterials. Die Qualität der Proben waren die wichtigste Grundlage für alle durchgeführten Experimente. Zudem erwies sich Gunther als sehr zu schätzender Ansprechpartner, dessen Diskussionen mich immer voran gebracht haben.

Einen ganz wesentlichen Anteil an dieser Arbeit hat auch die Arbeitsgruppe von Prof. Volkert am Institut für Materialphysik. Sie bot das nötige wissenschaftliche und zwischenmenschliche Umfeld, das für eine solche Arbeit unerlässlich ist. Ganz direkten Einfluss auf diese Arbeit hatten dabei Bahne Kapelle und Kodanda Ram Mangipudi. Bahne hat mit seinen quantitativen Untersuchungen wichtige Größen bestimmt, die direkt in die Modellbildung einfließen konnten. Bei Kodanda möchte ich mich für die FE Rechnungen der Rahmenstruktur bedanken.

Allen Mitarbeitern am Institut für Materialphysik gilt mein besonderer Dank, da sie mich in ihre Gemeinschaft aufgenommen und in jeder schwierigen Situation helfend unterstützt haben.

$\mathrm{Zu}$ guter Letzt gilt mein besonderer Dank meiner Familie. Allen vorran meine Frau und meine beiden Kinder, die mir jeden Tag aufs Neue liebevoll zur Seite standen. Meinen Eltern möchte ich an dieser Stelle dafür danken, dass sie mich auf diesem Weg begleitet haben und ich immer auf ihre Unterstützung bauen konnte. 



\title{
Curriculum Vitae
}

\author{
BURKHARD ROOS
}

\section{ADRESSE}

Fichtenweg 5

37077 Göttingen

Deutschland

Tel: +49-551-3879855

Email: burkhardroos@gmx.de

\section{PERSÖNLICHE DETAILS}

$\begin{array}{ll}\text { Geschlecht: } & \text { männlich } \\ \text { Geburtsdatum: } & 28.06 .1983 \\ \text { Geburtsort: } & \text { Lübeck, Deutschland } \\ \text { Staatsangehörigkeit: } & \text { deutsch } \\ \text { Familienstand: } & \text { verheiratet } \\ \text { Kinder: } & \text { Daria Joanna (geb.: 11.04.09) } \\ & \text { Bruno Julian (geb.: 10.08.11) }\end{array}$

\section{AusbiLdung}

08/1990-06/1993 Hochtor Grundschule in Neustadt in Holstein

08/1993-06/2002 Allgemeine Hochschulreife, Kreisgymnasium Neustadt in Holstein

10/2002-06/2007 Studium der Physik an der TU Clausthal

07/2004 Vordiplom

01/2007-09/2007 Diplomarbeit in der AG Atom- und Molekülphysik an Oberflächen, Institut für Physik und Physikalische Technologien, TU Clausthal

10/2007 Diplom

11/2007-10/2012 Wissenschaftlicher Angestellter am Institut für Materialphysik, Universität Göttingen

03/2012-08/2012 Elternzeit mit Sohn Bruno Julian 